PNNL-18597

WTP-RPT-200, Rev 0

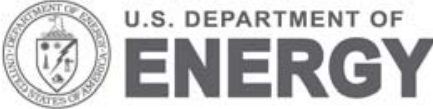

Prepared for the U.S. Department of Energy

under Contract DE-AC05-76RL01830

\title{
PEP Support: Laboratory Scale Leaching and Permeate Stability Tests
}

RL Russell

RA Peterson
DE Rinehart

WC Buchmiller

September 2009

Pacific Northwest

NATIONAL LABORATORY

Proudly Operated by Battelle Since 1965 


\section{PEP Support: Laboratory Scale Leaching and Permeate Stability Tests}

RL Russell

RA Peterson
DE Rinehart

WC Buchmiller

September 2009

Test Specification: 24590-PTF-TSP-RT-07-004, Rev 0 and 24590-PTF-TSP-RT-07-001, Rev 2

Test Plan: TP-RPP-WTP-509, Rev 0.5 and TP-WTP-PEP-044, Rev 0.2

Test Exceptions: 24590-WTP-TEF-RT-07-00016, 24590-WTP-TEF-RT-08-00013, 24590-WTP-TEF-RT-08-00014, 24590-WTP-TEF-RT-08-00015, 24590-WTP-TEF-RT-09-0001 Rev. 1, 24590-WTP-TEF-RT-09-00002

R\&T focus area: Pretreatment

Test Scoping Statement: None

Pacific Northwest National Laboratory

Richland, Washington 99352 
WTP-RPT-200, Rev 0

\section{Completeness of Testing}

This report describes the results of work and testing specified by Test Specifications, 24590-PTF-TSP-RT-07-001, Rev 2 and 24590-PTF-TSP-RT-07-004, Rev 0, and Test Plans TP-RPP-WTP-509, Rev. 0.5 and TP-WTP-PEP-044, Rev 0.2. The work followed the quality assurance requirements outlined in the Test Specification and Test Plan. The descriptions provided in this report are an accurate account of both the conduct of the work and the data collected. Test plan results are reported. Also reported are any unusual or anomalous occurrences that are different from expected results. The test results and this report has been reviewed and verified.

\section{Approved:}

W.EDhemen for GH Berman

Gordon H. Beeman, Manager WTP R\&T Support Project

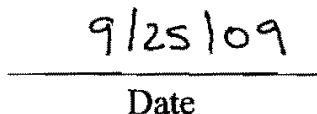

Date 


\section{Contents}

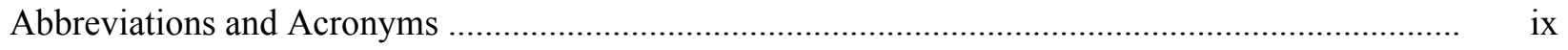

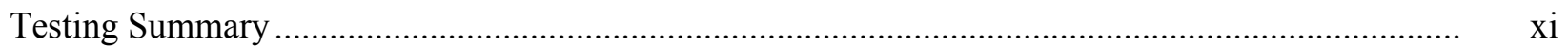

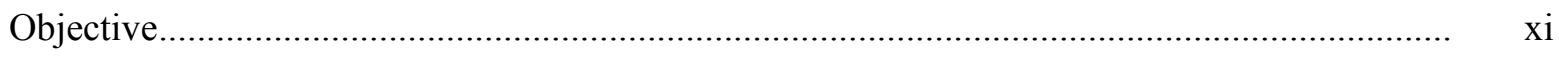

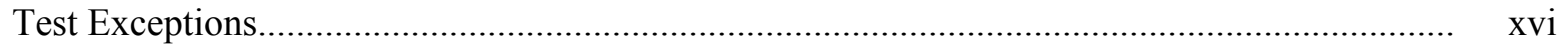

Results and Performance Against Success Criteria .............................................................. xvii

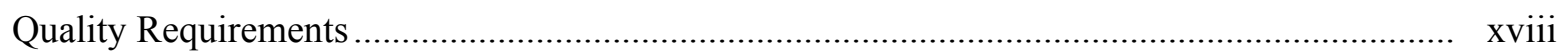

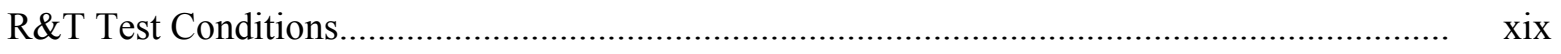

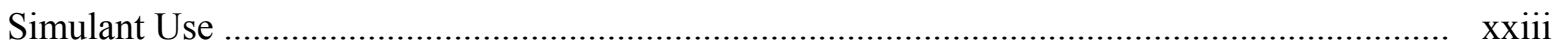

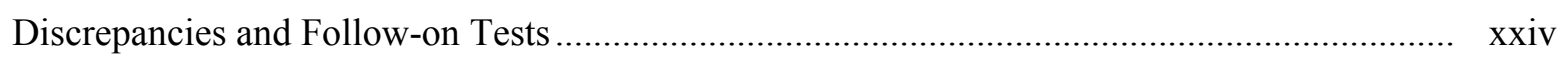

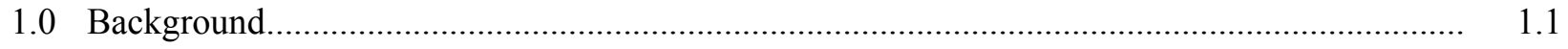

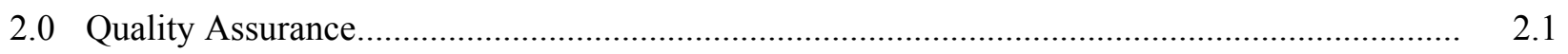

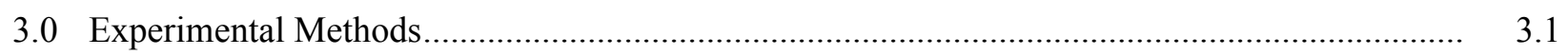

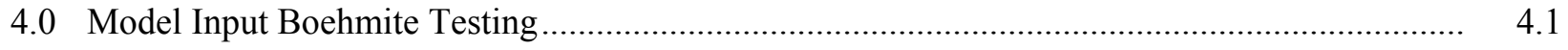

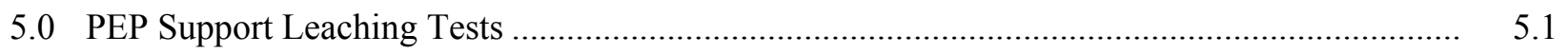

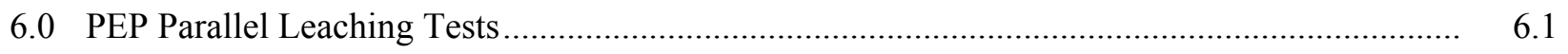

6.1 PEP Functional Test Caustic Leach ............................................................................. 6.1

6.2 PEP Test A Caustic Leach .................................................................................... 6.11

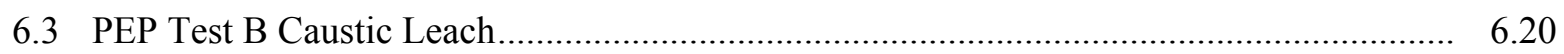

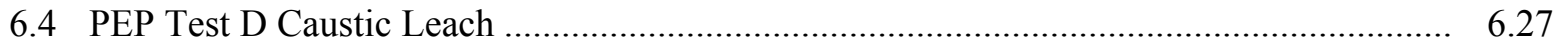

6.5 PEP Tests A, B, and D Oxidative Leach Parallel Laboratory Tests.................................. 6.35

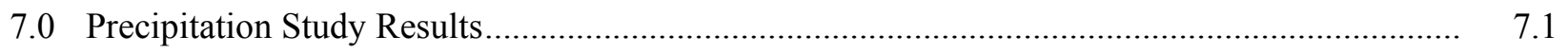

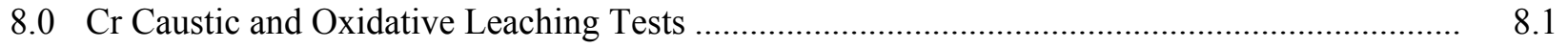

8.1 Chromium Characterization Leaching …............................................................... 8.1

8.2 Full Matrix Chromium Leaching Tests ..................................................................... 8.3

8.3 Chromium Oxide Leaching Tests …....................................................................... 8.5

8.4 Chromium Oxidative Leach Investigation Tests ....................................................... 8.8

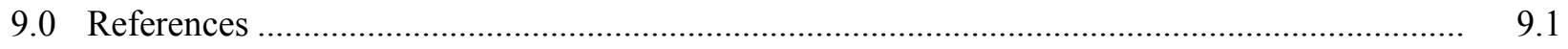

Appendix A: Boehmite Dissolution Model ….......................................................................... A.1

Appendix B: SOW for Chromium Hydroxide Preparation ......................................................... B. 1

Appendix C: Make Up of PEP Simulant and Component Composition and a Recipe for CrOOH

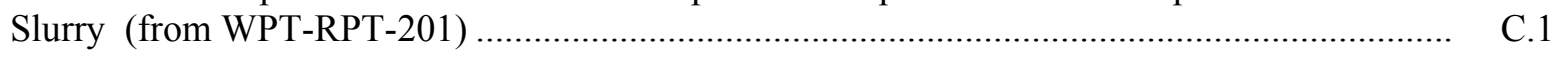




\section{Figures}

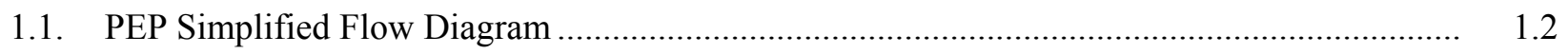

3.1. Schematic Drawing of the Caustic Leaching Test Setup................................................... 3.2

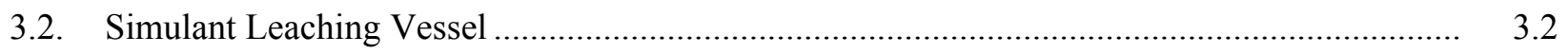

3.3. Simulant Leaching Vessel Stir Assembly .........................................................................

7.1. XRD Pattern of Crystals from Test A Wash Step \#3 (Sample Bottle \# 2341) .......................... 7.4

7.2. XRD Pattern of Crystals from Test A Wash \#29 (Sample Bottle \# 2367) ................................ 7.5

7.3. Air-Dried Crystals from Various Filtered Odd-Numbered Wash Step Permeate Samples ........ $\quad 7.6$

7.4. Even-Numbered Wash-Step Permeate Blend Crystals ........................................................ 7.7

7.5. More Even-Numbered Wash-Step Permeate Blend Crystals ................................................ 7.7

7.6. Air-Dried Crystals from the Even-Numbered Wash-Step Permeate Mixture ........................... $\quad 7.8$

7.7. Crystals Forming After Filtration of Even-Numbered Wash-Step Permeate Blend ................. $\quad 7.8$

7.8. Air-Dried Crystals from the 1:1 Wash-Step Permeate: Leachate Mixture ................................ 7.9

7.9. Crystals in Test A Initial Solids Concentration Permeate .................................................... 7.10

7.10. Polarized Light Picture of Sample Bottle \#D_00PF1_015_XX_5653_ARC_4 (post-caustic-leach dewater) with a Standard $\mathrm{Na}_{3} \mathrm{PO}_{4}$ Picture Beside it ................................. 7.11

7.11. Polarized Light Picture of Sample Bottle \#D_00PF1_015_XX_5444_ARC_4 (wash 8) with a Standard $\mathrm{Na}_{3} \mathrm{PO}_{4}$ Picture Under it

7.12. Polarized Light Picture of Sample Bottle \#D_00PF1_015_XX_5675_ARC_4 (wash 13) with a Standard $\mathrm{Na}_{3} \mathrm{PO}_{4}$ Picture (on the left) and a Standard $\mathrm{Na}_{2} \mathrm{C}_{2} \mathrm{O}_{4}$ Picture (on the right) Under it

7.13. Polarized Light Picture of Sample Bottle \#D_00PF1_015_XX_5476_ARC_4 (wash 40) with a Standard $\mathrm{Na}_{3} \mathrm{PO}_{4}$ Picture (on the left) and a Standard $\mathrm{Na}_{2} \mathrm{C}_{2} \mathrm{O}_{4}$ Picture (on the right) Under it

7.14. Particle Length Distribution of Sample Bottle \#D_00PF1_015_XX_5653_ARC_4 (post-caustic-leach dewater)

7.15. Particle Length Distribution of Sample Bottle \#D_00PF1_015_XX_5444_ARC_4 (wash 8)

7.16. Particle Length Distribution of Sample Bottle \#D_00PF1_015_XX_5675_ARC_4 (wash 13)

7.17. Particle Length Distribution of Sample Bottle \#D_00PF1_015_XX_5476_ARC_4 (wash 40). 


\section{Tables}

Table S.1. Test Objectives from TP-RPP-WTP-509, Rev. 0.5 ................................................................

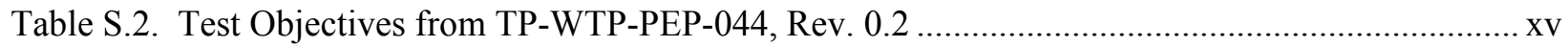

Table S.3. Test Exceptions for Test Plan TP-RPP-WTP-509 .............................................................. xvi

Table S.4. Test Exceptions for Test Plan TP-WTP-PEP-044 ..................................................................ii

Table S.5. Results and Performance Against Success Criteria of TP-RPP-WTP-509, Rev. 0.5 .............xvii

Table S.6. Results and Performance Against Success Criteria of TP-WTP-PEP-044, Rev. 0.2............xviii

Table S.7. R\&T Test Conditions for TP-RPP-WTP-509. Rev. 0.5 ...........................................................

Table S.8. R\&T Test Conditions for TP-WTP-PEP-044, Rev. 0.2 …................................................xii

1.1. Testing Objectives and Sample Identification Nomenclature ................................................. 1.3

4.1. Initial Set of Boehmite Model Input Tests .....................................................................

4.2. Second Set of Boehmite Model Input Tests ........................................................................

4.3. Dry-Solids Weight of Boehmite from the Model Input Leaching Tests ................................. 4.2

4.4. Al Results for Filtrate from the Initial Set of Boehmite Model Input Leaching Tests .............. $\quad 4.3$

4.5. Na Results for Filtrate from the Initial Set of Boehmite Model Input Leaching Tests ............. $\quad 4.4$

4.6. Al Results for Filtrate from the Second Set of Boehmite Model Input Leaching Tests............. $\quad 4.5$

4.7. Na Results for Filtrate from the Second Set of Boehmite Model Input Leaching Tests ........... $\quad 4.5$

5.1. Test Matrix for the PEP Support Leaching Tests ............................................................ 5.1

5.2. Test Matrix for the PEP Support Leaching Tests \#2 ........................................................ 5.1

5.3. Results for Solids from the PEP Support Leaching Tests .................................................. 5.3

5.4. Al Results for Filtrate from the PEP Support Leaching Tests................................................ 5.3

5.5. Cr Results for Filtrate from the PEP Support Leaching Tests ............................................. 5.4

5.6. $\mathrm{Na}$ Results for Filtrate from the PEP Support Leaching Tests .............................................. 5.4

5.7. Results for Solids from the PEP Support Leaching Tests \#2 ................................................. 5.5

5.8. Al Results for Filtrate from the PEP Support Leaching Tests \#2 ........................................... 5.5

5.9. Na Results for Filtrate from the PEP Support Leaching Tests \#2 ....................................... 5.6

6.2. Supernate Al Analysis from PEP Functional Test Caustic Leach ......................................... 6.3

6.3. Supernate Cr Analysis from PEP Functional Test Caustic Leach ......................................... 6.4

6.4. Supernate Na Analysis from PEP Functional Test Caustic Leach ........................................... 6.4

6.5. Slurry Analysis from PEP Functional Test Caustic Leach Test 1 ......................................... 6.5

6.6. Slurry Analysis from PEP Functional Test Caustic Leach Test 2 ....................................... 6.5

6.7. Slurry Analysis from PEP Functional Test Caustic Leach Test 3 ......................................... 6.6

6.8. Slurry Analysis from PEP Functional Test Caustic Leach Test 4 .......................................... 6.6

6.9. Anion Analysis of PL_LAB_CL_1 Supernate ................................................................. 6.7

6.10. Anion Analysis of PL_LAB_CL_2 Supernate ................................................................ 6.7 


\section{Tables}

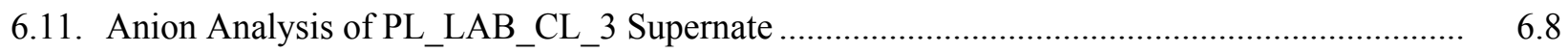

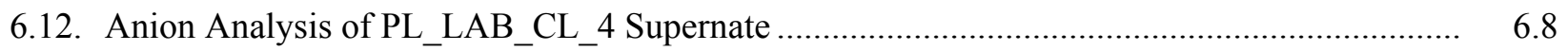

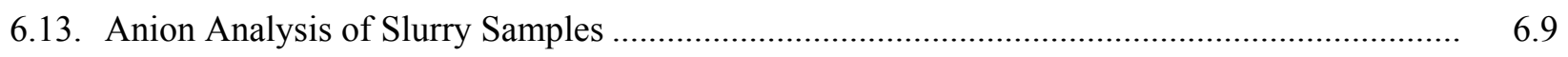

6.14. PEP Parallel Functional Test Density ....................................................................... 6.10

6.15. PEP Parallel Functional Test Physical Properties of Initial Slurries ...................................... 6.10

6.16. PEP Parallel Functional Test Free $\mathrm{OH}$ Concentration........................................................... 6.11

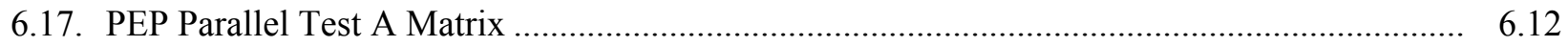

6.18. Supernate Al Analysis from PEP Parallel Test A Caustic Leach ........................................... 6.13

6.19. Supernate Cr Analysis from PEP Parallel Test A Caustic Leach ........................................... 6.13

6.20. Supernate Na Analysis from PEP Parallel Test A Caustic Leach ......................................... 6.14

6.21. Slurry Analysis from PEP Parallel Test A Caustic Leach Test 1 .......................................... 6.15

6.22. Slurry Analysis from PEP Parallel Test A Caustic Leach Test 2 ........................................ 6.15

6.23. Anion Analysis of A_LAB_CL_1 Supernate ....................................................................... 6.16

6.24. Anion Analysis of A_LAB_CL_2 Supernate ................................................................... 6.16

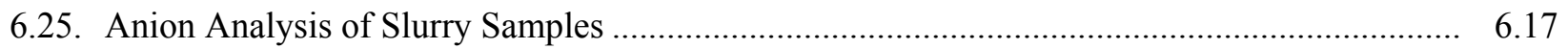

6.26. PEP Parallel Test A Free OH Supernate Concentration .................................................... 6.18

6.27. PEP Parallel Test A Slurry Free OH Concentration ............................................................. 6.18

6.28. PEP Parallel Test A Physical Properties for Test 1 ............................................................. 6.19

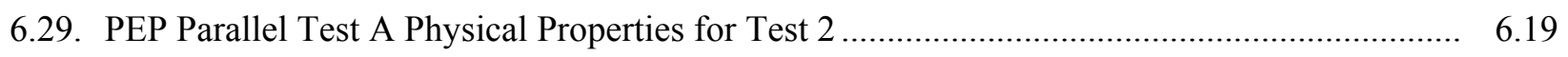

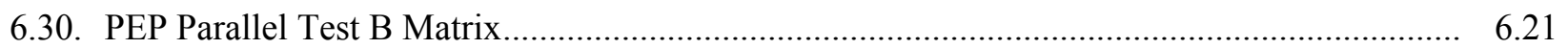

6.31. Supernate Al Analysis from PEP Parallel Test B Caustic Leach ........................................... 6.21

6.32. Supernate Cr Analysis from PEP Parallel Test B Caustic Leach ............................................ 6.22

6.33. Supernate Na Analysis from PEP Parallel Test B Caustic Leach ........................................... 6.22

6.34. Slurry Analysis from PEP Parallel Test B Caustic Leach Test 1 ........................................... 6.23

6.35. Slurry Analysis from PEP Parallel Test B Caustic Leach Test 2 …...................................... 6.23

6.36. Anion Analysis of B_LAB_CL_1 Supernate ................................................................. 6.24

6.37. Anion Analysis of B_LAB_CL_2 Supernate ............................................................... 6.24

6.38. Anion Analysis of Slurry Samples for Test B Caustic Leach ….......................................... 6.25

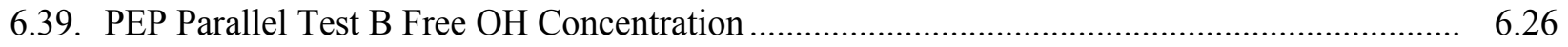

6.40. PEP Parallel Test B Physical Properties for Test 1 ............................................................. 6.26

6.41. PEP Parallel Test B Physical Properties for Test 2 ........................................................... 6.27

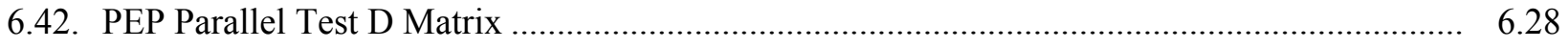

6.43. Supernate Al Analysis from PEP Parallel Test D Caustic Leach .......................................... 6.29

6.44. Supernate Cr Analysis from PEP Parallel Test D Caustic Leach ........................................... 6.29 


\section{Tables}

6.45. Supernate Na Analysis from PEP Parallel Test D Caustic Leach ........................................ 6.30

6.46. Slurry Analysis from PEP Parallel Test D Caustic Leach Test 1 ........................................ 6.30

6.47. Slurry Analysis from PEP Parallel Test D Caustic Leach Test 2 ........................................ 6.31

6.48. Anion Analysis of D_LAB_CL_1 Supernate ................................................................. 6.31

6.49. Anion Analysis of D_LAB_CL_2 Supernate .................................................................... 6.32

6.50. Anion Analysis of Slurry Samples for Test D Caustic Leach .............................................. 6.33

6.51. PEP Parallel Test D Free OH Concentration........................................................................ 6.34

6.52. PEP Parallel Test D Physical Properties for Test 1 ............................................................ 6.34

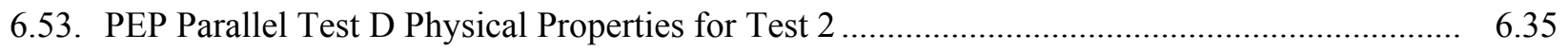

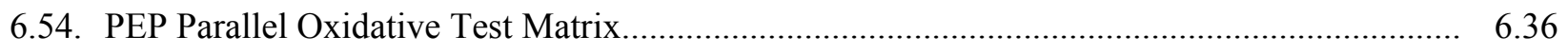

6.55. Supernate Al Analysis from PEP Parallel Test Oxidative Leach ......................................... 6.37

6.56. Supernate Cr Analysis from PEP Parallel Test Oxidative Leach ........................................... 6.37

6.57. Supernate Na Analysis from PEP Parallel Test Oxidative Leach ........................................... 6.38

6.58. Anion Analysis of A_LAB_OL_1 Supernate from Oxidative Leach Tests ............................ 6.38

6.59. Anion Analysis of B_LAB_OL_2 Supernate from Oxidative Leach Tests ............................ 6.38

6.60. Anion Analysis of D_LAB_OL_3 Supernate from Oxidative Leach Tests ............................ 6.39

6.61. Anion Analysis of D_LAB_OL_4 Supernate from Oxidative Leach Tests ............................ 6.39

6.62. Slurry Analysis from PEP Parallel Oxidative Leach Test A (Test 1)................................... 6.40

6.63. Slurry Analysis from PEP Parallel Oxidative Leach Test B (Test 2) .................................. 6.40

6.64. Slurry Analysis from PEP Parallel Oxidative Leach Test D, Excess $\mathrm{MnO}_{4}^{-}$(Test 3) .............. 6.41

6.65. Slurry Analysis from PEP Parallel Oxidative Leach Test D, Less $\mathrm{MnO}_{4}^{-}$(Test 4)................. 6.41

6.66. PEP Parallel Oxidative Test Supernate Density ................................................................. 6.42

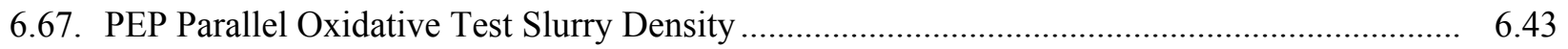

6.68. PEP Parallel Oxidative Test A_LAB_OL_1 Physical Properties.............................................. 6.44

6.69. PEP Parallel Oxidative Test B LAB_OL_2 Physical Properties ............................................. 6.44

6.70. PEP Parallel Test D LAB_OL_3 Physical Properties ......................................................... 6.45

6.71. PEP Parallel Oxidative Test D LAB_OL_4 Physical Properties........................................... 6.45

7.1. PEP Test A Wash Permeate Samples ...................................................................................

7.2. Weights of Filtered Solids in Odd-Numbered Test A Samples...............................................

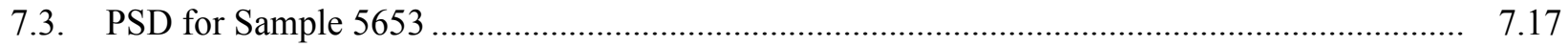

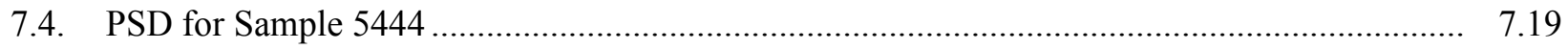

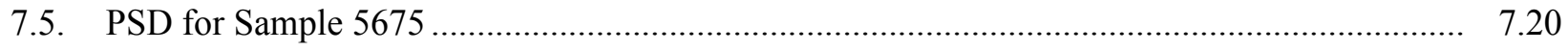

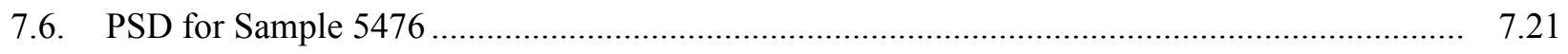

8.1. Chromium Compound Identification............................................................................ 8.2 


\section{Tables}

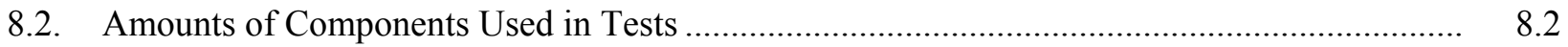

8.3. Cr Caustic Leaching Results from the Chromium Characterization Tests .............................. 8.2

8.4. Cr Oxidative Leaching Results from the Chromium Characterization Tests .......................... 8.3

8.5. Chromium Compound Identification for Full Matrix Testing ............................................. 8.3

8.6. Components Added to the Full Matrix Chromium Leaching Tests ......................................... 8.4

8.7. Al Results from the Full Matrix Chromium Caustic Leaching Tests ...................................... 8.4

8.8. Cr Results from the Full Matrix Chromium Caustic Leaching Tests ...................................... 8.4

8.9. Na Results from the Full Matrix Chromium Caustic Leaching Tests .................................... 8.5

8.10. Cr Results from the Full Matrix Chromium Oxidative Leaching Tests ................................. 8.5

8.11. Chromium Oxide Identification....................................................................................... 8.5

8.12. Components Added to the Chromium Oxide Leaching Tests .............................................. 8.6

8.13. Al Results from the Chromium Oxide Caustic Leaching Tests ............................................. 8.6

8.14. Cr Results from the Chromium Oxide Caustic Leaching Tests ................................................ 8.6

8.15. Na Results from the Chromium Oxide Caustic Leaching Tests ............................................ 8.7

8.16. Al Results from the Chromium Oxide Oxidative Leaching Tests.......................................... 8.7

8.17. Cr Results from the Chromium Oxide Oxidative Leaching Tests.......................................... 8.7

8.18. Na Results from the Chromium Oxide Oxidative Leaching Tests ........................................ 8.7

8.19. Chromium Investigative Leach Test Compound Identification ............................................. 8.8

8.20. Components Added to the Chromium Investigative Leach Tests ....................................... 8.9

8.21. Cr Results from the Chromium Investigative Caustic Leach Tests......................................... 8.9

8.22. Cr Results from the Chromium Investigative Oxidative Leach Tests ...................................... 8.9 


\title{
Abbreviations and Acronyms
}

\author{
AFA anti-foaming agent \\ APEL Applied Process Engineering Laboratory \\ ASME American Society of Mechanical Engineers \\ BNI Bechtel National, Incorporated \\ CD Coriolis densitometer \\ CUF crossflow ultrafiltration system (also known as cell unit filter) \\ DI deionized (water) \\ DOE U.S. Department of Energy \\ EFRT External Flowsheet Review Team \\ HLW high-level waste \\ IC ion chromatography \\ ICP-AES inductively coupled plasma-atomic emission spectroscopy \\ ICP-OES inductively coupled plasma-optical emission spectrometry \\ LAW low-activity waste \\ LCS laboratory control sample \\ PEP Pretreatment Engineering Platform \\ PLM polarized light microscopy \\ PMP poly-methyl pentene \\ PNNL Pacific Northwest National Laboratory \\ PSD particle-size distribution \\ PTF Pretreatment Facility \\ QA quality assurance \\ QAM Quality Assurance Manual \\ QAP quality assurance program
}




$\begin{array}{ll}\text { QARD } & \text { Quality Assurance Requirements and Descriptions (document) } \\ \text { QC } & \text { quality control } \\ \text { rpm } & \text { revolutions per minute } \\ \text { RPP } & \text { River Protection Project } \\ \text { R\&T } & \text { research and technology } \\ \text { SBMS } & \text { Standards-Based Management System } \\ \text { SEM } & \text { scanning electron microscopy } \\ \text { SOW } & \text { statement of work } \\ \text { SWRI } & \text { Southwest Research Institute } \\ \text { TC } & \text { thermocouple } \\ \text { TRU } & \text { transuranic elements } \\ \text { UDS } & \text { undissolved solids } \\ \text { UFP } & \text { ultrafiltration process } \\ \text { UV-Vis } & \text { ultraviolet-visible } \\ \text { WCS } & \text { wet centrifuged solids } \\ \text { WTP } & \text { Hanford Tank Waste Treatment and Immobilization Plant } \\ \text { XRD } & \text { X-ray diffraction } \\ & \end{array}$




\section{Testing Summary}

This report documents results from a variety of activities requested by the Hanford Tank Waste Treatment and Immobilization Plant (WTP). The activities related to caustic leaching, oxidative leaching, permeate precipitation behavior of waste as well as chromium $(\mathrm{Cr})$ leaching are:

- Model Input Boehmite Leaching Tests

- Pretreatment Engineering Platform (PEP) Support Leaching Tests

- PEP Parallel Leaching Tests

- Precipitation Study Results

- Cr Caustic and Oxidative Leaching Tests.

Leaching test activities using the PEP simulant provided input to a boehmite dissolution model and determined the effect of temperature on mass loss during caustic leaching, the reaction rate constant for the boehmite dissolution, and the effect of aeration in enhancing the chromium dissolution during caustic leaching. Other tests were performed in parallel with the PEP tests to support the development of scaling factors for caustic and oxidative leaching. Another study determined if precipitate formed in the wash solution after the caustic leach in the PEP. Finally, the leaching characteristics of different chromium compounds under different conditions were examined to determine the best one to use in further testing.

The results of the leaching tests to support the development of a boehmite dissolution model were completed in accordance with Test Plan TP-RPP-WTP-509, Rev 0.5, ${ }^{(a)}$ prepared and approved in response to Test Specification 24590-PTF-TSP-RT-07-004, Rev $0^{(\mathrm{b})}$ in Sections 4.0, 5.0, and 8.0.

The results of the PEP parallel testing in Sections 6.0 and 7.0 were performed in accordance with Test Plan TP-WTP-PEP-044, Rev 0.2, ${ }^{(c)}$ prepared and approved in response to Test Specification 24590-WTP-TSP-RT-07-001, Rev 2 (Huckaby 2008).

\section{Objective}

The test objectives for the work addressed in TP-RPP-WTP-509, Rev $0.5^{(\mathrm{a})}$ are summarized in Table S.1 along with a discussion of how the objectives were met. The test objectives for the work addressed in TP-WTP-PEP-044, Rev $0.2^{\text {(b) }}$ are summarized in Table S.2 along with a discussion of how the objectives were met.

(a) RC Daniel and RW Shimskey. 2007. "Test Plan for Simulant Testing in Support of Phase I Demonstration of the Ultrafiltration and Leaching Processes in the Integrated Test Facility.” TP-RPP-WTP-509, Rev. 0.5, Pacific Northwest National Laboratory, Richland, Washington.

(b) PS Sundar. 2007. Simulant Testing in Support of Phase I Demonstration of the Ultrafiltration and Leaching Processes in the Integrated Test Facility. WTP Project Doc. No. 24590-WTP-TSP-RT-07-004, Rev. 0.

(c) RL Russell. 2008. "Test Plan for the PEP Parallel Laboratory Testing." TP-WTP-PEP-044, Rev. 0, Pacific Northwest National Laboratory, Richland, Washington. 
Table S.1. Test Objectives from TP-RPP-WTP-509, Rev. 0.5

\begin{tabular}{|c|c|c|c|}
\hline \multicolumn{2}{|c|}{ Test Objective } & \multirow{2}{*}{$\begin{array}{c}\begin{array}{c}\text { Objective } \\
\text { Met? (Y/N) }\end{array} \\
\text { NA }\end{array}$} & \multirow{2}{*}{$\begin{array}{l}\text { Discussion } \\
\text { This objective was addressed in report } \\
\text { WTP-RPT-184, Rev. } 1 .\end{array}$} \\
\hline & $\begin{array}{l}\text { Determine the effect of initial } \\
\text { aluminate ion concentration on } \\
\text { the rate of boehmite leaching in } \\
\text { caustic solutions and in the } \\
\text { presence of soluble anions in a } \\
\text { waste. The anions to be } \\
\text { considered are those that are } \\
\text { typically present in the Hanford } \\
\text { Tank Farm wastes in significant } \\
\text { amounts. This includes } \\
\text { carbonate, free-hydroxide, nitrate, } \\
\text { nitrite, oxalate, phosphate, and } \\
\text { sulfate. }\end{array}$ & & \\
\hline 2) & $\begin{array}{l}\text { Determine the sensitivity of the } \\
\text { rate of dissolution of boehmite to } \\
\text { soluble anions through a limited } \\
\text { number of laboratory tests. The } \\
\text { anions to be considered are those } \\
\text { that are typically present in the } \\
\text { Hanford Tank Farm wastes in } \\
\text { significant amounts. This } \\
\text { includes carbonate, free } \\
\text { hydroxide, nitrate, nitrite, oxalate, } \\
\text { phosphate, and sulfate. }\end{array}$ & NA & $\begin{array}{l}\text { As of March 20, 2008, Pacific Northwest National } \\
\text { Laboratory (PNNL) has been released from this } \\
\text { objective by Test Exception } \\
24590 \text {-WTP-TEF-RT-07-00016. }\end{array}$ \\
\hline & $\begin{array}{l}\text { Determine the effect of scaling the } \\
\text { length of the ultrafilter element } \\
\text { from } 2 \mathrm{ft} \text { to } 8 \mathrm{ft} \text { on the filtrate flux } \\
\text { over the expected operating range } \\
\text { of the ultrafilter using the Cell } \\
\text { Unit Filter. }\end{array}$ & NA & $\begin{array}{l}\text { This objective was addressed in report } \\
\text { WTP-RPT-168, Rev. } 0 .\end{array}$ \\
\hline 4) & $\begin{array}{l}\text { Use an 8-ft-long filter element in } \\
\text { the CUF unit to determine the } \\
\text { effect of temperature on the } \\
\text { filtration of a waste simulant over } \\
\text { the range of temperature } \\
\text { conditions for the leaching } \\
\text { processes. }\end{array}$ & NA & $\begin{array}{l}\text { This objective was addressed in report } \\
\text { WTP-RPT-168, Rev. } 0 .\end{array}$ \\
\hline
\end{tabular}


Table S.1. Test Objectives from TP-RPP-WTP-509, Rev. 0.5

\begin{tabular}{|c|c|c|c|}
\hline \multicolumn{2}{|c|}{ Test Objective } & \multirow{2}{*}{$\begin{array}{c}\begin{array}{c}\text { Objective } \\
\text { Met? (Y/N) }\end{array} \\
\text { NA }\end{array}$} & \multirow{2}{*}{$\begin{array}{l}\text { Discussion } \\
\text { This objective was addressed in report } \\
\text { WTP-RPT-183, Rev. } 0 .\end{array}$} \\
\hline & $\begin{array}{l}\text { Use a 2-ft-long filter element in } \\
\text { the CUF unit to evaluate the effect } \\
\text { of the fine particle fraction in the } \\
\text { ultrafiltration simulant on fouling } \\
\text { of the filter element over the } \\
\text { range of concentrations of } \\
\text { operating solids. The fine particle } \\
\text { fraction is defined as those } \\
\text { particles with diameters smaller } \\
\text { than the } 10^{\text {th }} \text { percentile (i.e., the } \\
\text { dp10) of the particle-size number } \\
\text { distribution. }\end{array}$ & & \\
\hline 6) & $\begin{array}{l}\text { Perform various simulant aging } \\
\text { tests to understand the changes } \\
\text { that may occur to the simulant in } \\
\text { storage and to ensure the } \\
\text { adequacy of the simulant for use } \\
\text { in the PEP. }\end{array}$ & NA & $\begin{array}{l}\text { This objective was addressed in report } \\
\text { WTP-RPT-198, Rev. } 0 .\end{array}$ \\
\hline 7) & $\begin{array}{l}\text { Perform Cr-simulant leaching } \\
\text { tests to establish that the } \\
\text { Cr-simulant from the larger batch } \\
\text { exhibits similar or better leaching } \\
\text { behavior than the initial trial batch } \\
\text { during caustic and oxidative } \\
\text { leaching operations. }\end{array}$ & $\mathrm{Y}$ & $\begin{array}{l}\text { Cr-simulant leaching tests were performed with a } \\
\text { caustic leaching test at } 100^{\circ} \mathrm{C} \text { for } 24 \text { hours, followed } \\
\text { by an oxidative leach at room temperature for } \\
6 \text { hours. These results are presented in Section 8.0. }\end{array}$ \\
\hline 8) & $\begin{array}{l}\text { Perform leaching tests to } \\
\text { determine the mass loss, and } \\
\text { aluminum and chromium } \\
\text { dissolution rates during caustic } \\
\text { leaching under varying } \\
\text { temperature processing conditions } \\
\text { without aeration in both } \\
\text { UFP-1A/B and UFP-2A/B vessels } \\
\text { as well as to measure the effect of } \\
\text { aeration on chromium leaching in } \\
\text { UFP-2A/B. }\end{array}$ & $\mathrm{Y}$ & $\begin{array}{l}\text { These tests were performed by placing the PEP } \\
\text { simulant containing the required amount of solids } \\
\text { into the reaction vessel and adding the needed } \\
\text { amount of caustic and Cr slurry. It was then heated } \\
\text { to the required temperature and allowed to react. } \\
\text { One test was aerated at } \sim 85-\mathrm{mL} / \text { min according to the } \\
\text { rotometer reading at laboratory conditions. These } \\
\text { results are presented in Section } 5.0 \text {. }\end{array}$ \\
\hline & $\begin{array}{l}\text { Perform leaching tests to develop } \\
\text { an accurate model for the } \\
\text { dissolution of boehmite. }\end{array}$ & $\mathrm{Y}$ & $\begin{array}{l}\text { Caustic leaching tests were performed with boehmite } \\
\text { under varying conditions of temperature, amount of } \\
\text { gibbsite, and boehmite source. From these tests, a } \\
\text { model for the dissolution of boehmite was developed } \\
\text { and is presented in Appendix A. The caustic leach } \\
\text { results used to develop the model are presented in } \\
\text { Section 4.0. }\end{array}$ \\
\hline
\end{tabular}


Table S.1. Test Objectives from TP-RPP-WTP-509, Rev. 0.5

\begin{tabular}{|ll|c|l||}
\hline Test Objective & $\begin{array}{c}\text { Objective } \\
\text { Met? (Y/N) }\end{array}$ & Discussion \\
\hline 10$)$ & $\begin{array}{l}\text { Perform leaching tests to verify } \\
\text { the effect of aluminate ions on the } \\
\text { performance of the boehmite } \\
\text { component B3 during caustic } \\
\text { leach at temperatures lower than } \\
100^{\circ} \mathrm{C} \text { and to determine the effect } \\
\text { of temperature on the dissolution } \\
\text { rate of boehmite component B7. }\end{array}$ & NA & $\begin{array}{l}\text { This objective was addressed in report } \\
\text { WTP-RPT-184, Rev. 1. }\end{array}$ \\
\hline 11) $\begin{array}{l}\text { Perform leaching tests to } \\
\text { determine the extent of boehmite } \\
\text { dissolution one would expect } \\
\text { under leaching conditions during } \\
\text { the planned testing in PEP. }\end{array}$ & Y & $\begin{array}{l}\text { These tests were performed by blending the PEP } \\
\text { simulant components together in different ratios and } \\
\text { then leaching them at } 80^{\circ} \mathrm{C} \text { to } 100^{\circ} \mathrm{C} \text { to determine } \\
\text { the amount of boehmite leached under similar PEP } \\
\text { operating conditions. These results are presented in } \\
\text { Section 5.0. }\end{array}$ \\
\hline
\end{tabular}


Table S.2. Test Objectives from TP-WTP-PEP-044, Rev. 0.2

\begin{tabular}{|c|c|c|c|}
\hline \multicolumn{2}{|c|}{ Test Objective } & \multirow{2}{*}{$\begin{array}{c}\begin{array}{c}\text { Objective } \\
\text { Met? (Y/N) }\end{array} \\
\text { NA }\end{array}$} & \multirow{2}{*}{$\begin{array}{l}\text { Discussion } \\
\text { This objective was addressed in reports } \\
\text { WTP-RPT-186, Rev. 0, WTP-RPT-188, Rev. 0, and } \\
\text { WTP-RPT-197, Rev. 0. The laboratory-scale } \\
\text { leaching results to support the development of } \\
\text { scaling factors are presented in Section } 6.0 \text {. }\end{array}$} \\
\hline & $\begin{array}{l}\text { Establish scale factors between } \\
\text { laboratory process measurements } \\
\text { (e.g., leach factors, filter fluxes) } \\
\text { and those of the PEP. }\end{array}$ & & \\
\hline & $\begin{array}{l}\text { Maximize the comparability of the } \\
\text { laboratory and PEP process } \\
\text { measurements and simplify the } \\
\text { development of the scaling } \\
\text { relationships. Slurry samples will } \\
\text { be collected from the PEP at } \\
\text { appropriate points during the } \\
\text { testing, transported to a laboratory, } \\
\text { and subjected to analogous } \\
\text { laboratory testing. Filter flux } \\
\text { measurements for slurries with low } \\
\text { and high solids concentrations, } \\
\text { caustic leaching, and oxidative } \\
\text { leaching will each be examined in } \\
\text { this fashion. }\end{array}$ & NA & $\begin{array}{l}\text { This objective was addressed in reports } \\
\text { WTP-RPT-186, Rev. 0, WTP-RPT-188, Rev. 0, and } \\
\text { WTP-RPT-197, Rev. 0. The laboratory-scale } \\
\text { leaching results to support the development of } \\
\text { scaling factors are presented in Section } 6.0 .\end{array}$ \\
\hline 3) & $\begin{array}{l}\text { Permeate precipitation testing will } \\
\text { be performed to evaluate the } \\
\text { propensity of the Phase I simulant } \\
\text { permeates to precipitate solids. }\end{array}$ & $\mathrm{Y}$ & $\begin{array}{l}\text { This objective is addressed in Section 7.0. It was } \\
\text { found that the majority of the wash solutions formed } \\
\text { precipitates that appeared to be sodium oxalate and } \\
\text { sodium phosphate, which were at their solubility } \\
\text { limits but not within the range of feed concentrations } \\
\text { expected during pretreatment of the actual HLW. }\end{array}$ \\
\hline 4) & $\begin{array}{l}\text { Develop an understanding of the } \\
\text { post-filtration precipitation } \\
\text { phenomenon. }\end{array}$ & Y & $\begin{array}{l}\text { This objective is addressed in Section 7.0. It was } \\
\text { found that the precipitates are mainly sodium oxalate } \\
\text { and sodium phosphate, which were at their solubility } \\
\text { limits but not within the range of feed concentrations } \\
\text { expected during pretreatment of the actual HLW. }\end{array}$ \\
\hline 5) & $\begin{array}{l}\text { Develop empirical information } \\
\text { needed to 1) understand the } \\
\text { operating bounds in concentration } \\
\text { and temperature to avoid } \\
\text { post-filtration precipitation in the } \\
\text { caustic leach process solutions, and } \\
\text { 2) identify and assess a need for a } \\
\text { change to the flowsheet, if } \\
\text { required, to improve process } \\
\text { operability. }\end{array}$ & NA & $\begin{array}{l}\text { These results will be presented in a report that is yet } \\
\text { to be released. }\end{array}$ \\
\hline
\end{tabular}




\section{Test Exceptions}

Four test exceptions were issued for Test Plan TP-RPP-WTP-509. These test exceptions are summarized in Table S.3 along with a brief description of how each exception impacted existing objectives and the test plan scope.

There were two test exceptions issued for Test Plan TP-WTP-PEP-044. These test exceptions are summarized in Table S.4 along with a brief description of how each exception impacted existing objectives and the test plan scope.

Table S.3. Test Exceptions for Test Plan TP-RPP-WTP-509

\begin{tabular}{|c|c|}
\hline List Test Exceptions & Describe Test Exceptions \\
\hline $\begin{array}{l}\text { 24590-WTP-TEF-RT-07-00016, Rev. } 0 \\
\text { (ICN-TP-RPP-WTP-509_R0.1) }\end{array}$ & $\begin{array}{l}\text { This test exception released PNNL from test Objective } 2 \text { (see } \\
\text { Table S.1). }\end{array}$ \\
\hline $\begin{array}{l}\text { 24590-WTP-TEF-RT-08-00013, Rev. } 0 \\
\text { (ICN-TP-RPP-WTP-509_R0.2) }\end{array}$ & $\begin{array}{l}\text { This test exception did not affect any existing test plan objectives. It } \\
\text { added test objectives concerned with 1) aging of the PEP simulants } \\
\text { during storage, and 2) leaching of the chromium simulant. These are } \\
\text { Objectives } 6 \text { and } 7 \text { in Table S.1. }\end{array}$ \\
\hline $\begin{array}{l}\text { 24590-WTP-TEF-RT-08-00014, Rev. } 0 \\
\text { (ICN-TP-RPP-WTP-509_R0.3 and } \\
\text { ICN-TP-RPP-WTP-509_R0.4) }\end{array}$ & $\begin{array}{l}\text { This test exception both affected existing test objectives and added new } \\
\text { test objectives. Tests associated with Objective } 4 \text { were modified } \\
\text { slightly in response to this test exception. Specifically, the temperatures } \\
\text { to study the filter performance were changed from } 25^{\circ} \mathrm{C}, 45^{\circ} \mathrm{C} \text {, and } \\
65^{\circ} \mathrm{C} \text { to } 25^{\circ} \mathrm{C}, 35^{\circ} \mathrm{C} \text {, and } 45^{\circ} \mathrm{C} \text {. In addition, a new objective concerned } \\
\text { with the influence of temperature and aeration on caustic leaching } \\
\text { processes was added to TP-RPP-WTP-509. These are summarized in } \\
\text { Objective } 8 \text { in Table S.1. }\end{array}$ \\
\hline $\begin{array}{l}\text { 24590-WTP-TEF-RT-08-00015, Rev. } 0 \\
\text { (ICN-TP-RPP-WTP-509_R0.5) }\end{array}$ & $\begin{array}{l}\text { This test exception did not affect any existing objectives in } \\
\text { TP-RPP-WTP-509. It added three new test objectives concerned with } \\
\text { 1) in-depth assessment of the boehmite leaching kinetics in the presence } \\
\text { of dissolved aluminate in significant concentration, and 2) the extent of } \\
\text { leaching under planned PEP operating conditions. These are } \\
\text { summarized in Objectives } 9,10 \text {, and } 11 \text { in Table S.1. }\end{array}$ \\
\hline
\end{tabular}


Table S.4. Test Exceptions for Test Plan TP-WTP-PEP-044

\begin{tabular}{|l|l||}
\hline \hline List Test Exceptions & Describe Test Exceptions \\
\hline $\begin{array}{l}\text { 24590-WTP-TEF-RT-09-00001, Rev. 1 } \\
\text { (ICN-TP-WTP-PEP-044_R0.2) }\end{array}$ & $\begin{array}{l}\text { This test exception did not affect any existing test plan objectives. It } \\
\text { added test objectives concerned with 1) understanding the operating } \\
\text { bounds in concentration and temperature to avoid post-filtration } \\
\text { precipitation in the caustic leach process solutions, and 2) identifying } \\
\text { and assessing a need for a change to the flowsheet, if required, to } \\
\text { improve process operability. This is Objective 5 in Table S.2. }\end{array}$ \\
\hline $\begin{array}{l}\text { 24590-WTP-TEF-RT-09-00002, Rev. 0 } \\
\text { (ICN-TP-WTP-PEP-044_R0.1) }\end{array}$ & $\begin{array}{l}\text { This test exception did not affect any existing test plan objectives. It } \\
\text { added test objectives concerned with developing an understanding of the } \\
\text { post-filtration precipitation process. This is Objective } 4 \text { in Table S.2. }\end{array}$ \\
\hline
\end{tabular}

\section{Results and Performance Against Success Criteria}

The success criteria described in TP-RPP-WTP-509, Rev. 0.5, which are listed in Table S.5, do not apply to this report. The success criterion described in TP-WTP-PEP-044, Rev 0.2 that this testing will meet is presenting results that allow a scale-up factor from the laboratory to the PEP testing to be generated. The work described in this report supplies the laboratory-scale leaching results for the development of this scale-up factor. None of the other success criteria, which are listed in Table S.6, are met by this report.

Table S.5. Results and Performance Against Success Criteria of TP-RPP-WTP-509, Rev. 0.5

\begin{tabular}{|c|c|}
\hline List Success Criteria & $\begin{array}{l}\text { Explain How the Tests Did or Did Not } \\
\text { Meet the Success Criteria }\end{array}$ \\
\hline $\begin{array}{l}\text { 1. Develop empirical information that allows } \\
\text { determination of the effect of initial aluminate ion } \\
\text { concentration on the kinetics of boehmite leaching } \\
\text { in a waste simulant. }\end{array}$ & $\begin{array}{l}\text { This criterion is addressed in report WTP-RPT-184, } \\
\text { Rev. } 1 .\end{array}$ \\
\hline $\begin{array}{l}\text { 2. Determine the sensitivity of boehmite leaching to } \\
\text { carbonate, free-hydroxide, nitrate, nitrite, oxalate, } \\
\text { phosphate, and sulfate anions in a waste-simulant } \\
\text { solution. }\end{array}$ & $\begin{array}{l}\text { As of March 20, 2008, PNNL was released from this } \\
\text { objective by Test Exception } \\
\text { 24590-WTP-TEF-RT-07-00016. }\end{array}$ \\
\hline $\begin{array}{l}\text { 3. Determine the effect of scaling the length of the } \\
\text { ultrafilter element from } 2 \mathrm{ft} \text { to } 8 \mathrm{ft} \text { on the } \\
\text { performance of the filter over the expected process } \\
\text { operating range in transmembrane pressure, axial } \\
\text { velocity, and ultrafiltration temperature. }\end{array}$ & $\begin{array}{l}\text { This criterion is addressed in report WTP-RPT-168, } \\
\text { Rev. } 0 .\end{array}$ \\
\hline $\begin{array}{l}\text { 4. Determine the effect of temperature on the filtration } \\
\text { flux for the waste simulant over the range of solids } \\
\text { concentrations and temperature conditions for the } \\
\text { leaching processes. }\end{array}$ & $\begin{array}{l}\text { This criterion is addressed in report WTP-RPT-168, } \\
\text { Rev. } 0 .\end{array}$ \\
\hline $\begin{array}{l}\text { 5. Determine the effect of fine-particle concentration } \\
\text { on the propensity of the waste simulant to foul the } \\
\text { ultrafilter element over the range of concentrations } \\
\text { of operating solids in the waste simulant. }\end{array}$ & $\begin{array}{l}\text { This criterion is addressed in report WTP-RPT-183, } \\
\text { Rev. } 0 .\end{array}$ \\
\hline
\end{tabular}


Table S.6. Results and Performance Against Success Criteria of TP-WTP-PEP-044, Rev. 0.2

\begin{tabular}{|c|c|c|}
\hline \multicolumn{2}{|c|}{ List Success Criteria } & \multirow{2}{*}{$\begin{array}{l}\text { Explain How the Tests Did or Did Not } \\
\text { Meet the Success Criteria } \\
\text { Test results were generated that were used in generating } \\
\text { a scale-up factor from the laboratory testing to the PEP } \\
\text { testing. The laboratory testing data are given in } \\
\text { Section 5.0, and the scale-up factor to the PEP testing is } \\
\text { discussed in reports WTP-RPT-186, Rev. } 0 \text { and } \\
\text { WTP-RPT-188, Rev. 0, and will be further addressed in } \\
\text { WTP-RPT-197, Rev. } 0 \text {. }\end{array}$} \\
\hline & $\begin{array}{l}\text { Generate testing results that allow a scale-up factor } \\
\text { from the laboratory testing to the PEP testing to be } \\
\text { generated. }\end{array}$ & \\
\hline & $\begin{array}{l}\text { Determine the mineralogy of the precipitate phase, } \\
\text { precipitate composition, and the solution phase } \\
\text { saturation composition for the composite samples } \\
\text { from demonstration Test A. }\end{array}$ & $\begin{array}{l}\text { This criterion is not addressed in this report and will be } \\
\text { addressed in WTP-RPT-205, Rev. } 0 \text {. }\end{array}$ \\
\hline & $\begin{array}{l}\text { Determine the rate at which the anions-phosphate, } \\
\text { oxalate, sulfate, silicate, and fluoride-approach } \\
\text { equilibrium solution composition (saturation } \\
\text { concentration) in post-caustic-leach slurry at } 25^{\circ} \mathrm{C} \\
\text { before filtration. }\end{array}$ & $\begin{array}{l}\text { This criterion is not addressed in this report and will be } \\
\text { addressed in WTP-RPT-205, Rev. } 0 \text {. }\end{array}$ \\
\hline & $\begin{array}{l}\text { Identify precipitates formed at ambient temperature } \\
\text { in the presence of phosphate, oxalate, sulfate, } \\
\text { silicate, and fluoride anions in the } \\
\text { post-caustic-leachate solution. Also, determine } \\
\text { particle-size distribution (PSD), crystal shape and } \\
\text { habit (morphology), quantity, and the settling rate } \\
\text { of precipitates formed. }\end{array}$ & $\begin{array}{l}\text { This criterion is not addressed in this report and will be } \\
\text { addressed in WTP-RPT-205, Rev. } 0 \text {. }\end{array}$ \\
\hline & $\begin{array}{l}\text { Determine the dilution required to redissolve the } \\
\text { post-filtration precipitate through incremental } \\
\text { dilution of the slurry with water at } 25^{\circ} \mathrm{C} \text {. }\end{array}$ & $\begin{array}{l}\text { This criterion is not addressed in this report and will be } \\
\text { addressed in WTP-RPT-205, Rev. } 0 \text {. }\end{array}$ \\
\hline 6) & $\begin{array}{l}\text { Determine solution supersaturation in the } \\
\text { post-caustic-leach filtrate during the dewatering } \\
\text { period, based on the samples collected at discrete } \\
\text { times during Test B in the PEP. The } \\
\text { supersaturation shall be expressed as both the } \\
\text { centrifuged volume fraction and as weight fraction } \\
\text { of the slurry sample. Also, determine the } \\
\text { temperature at which the precipitate goes into total } \\
\text { solution. }\end{array}$ & $\begin{array}{l}\text { This criterion is not addressed in this report and will be } \\
\text { addressed in WTP-RPT-205, Rev. } 0 \text {. }\end{array}$ \\
\hline & $\begin{array}{l}\text { Determine the effects of blending during the } \\
\text { post-caustic-leach dewatering and wash periods in } \\
\text { Test B in the PEP. }\end{array}$ & $\begin{array}{l}\text { This criterion is not addressed in this report and will be } \\
\text { addressed in WTP-RPT-205, Rev. } 0 \text {. }\end{array}$ \\
\hline
\end{tabular}

\section{Quality Requirements}

The PNNL quality assurance (QA) program is based upon the requirements as defined in the U.S. Department of Energy (DOE) Order 414.1C, Quality Assurance, and 10 CFR 830, Energy/Nuclear Safety 
Management, Subpart A-Quality Assurance Requirements (a.k.a. the Quality Rule). PNNL has chosen to implement the following consensus standards in a graded approach:

- ASME NQA-1-2000, Quality Assurance Requirements for Nuclear Facility Applications, Part 1, Requirements for Quality Assurance Programs for Nuclear Facilities.

- ASME NQA-1-2000, Part II, Subpart 2.7, Quality Assurance Requirements for Computer Software for Nuclear Facility Applications.

- ASME NQA-1-2000, Part IV, Subpart 4.2, Graded Approach Application of Quality Assurance Requirements for Research and Development.

The procedures necessary to implement the requirements are documented through PNNL's Standards-Based Management System (SBMS).

PNNL implements the RPP-WTP quality requirements by performing work in accordance with the River Protection Project-Hanford Tank Waste Treatment and Immobilization Plant Support Program (RPP-WTP) Quality Assurance Plan (RPP-WTP-QA-001, QAP). Work was performed to the quality requirements of NQA-1-1989 Part I, Basic and Supplementary Requirements, NQA-2a-1990, Part 2.7, and DOE/RW-0333P, Rev 13, Quality Assurance Requirements and Descriptions (QARD), as applicable. These quality requirements are implemented through the River Protection Project-Waste Treatment Plant Support Program (RPP-WTP) Quality Assurance Manual (RPP-WTP-QA-003, QAM). The requirements of DOE/RW-0333P, Rev 13, Quality Assurance Requirements and Descriptions (QARD) and 10 CFR 830, Subpart A, were not required for this work.

The RPP-WTP addresses internal verification and validation activities by conducting an independent technical review of the final data report in accordance with RPP-WTP's procedure QA-RPP-WTP-604. This review procedure is part of PNNL's RPP-WTP Quality Assurance Manual (RPP-WTP-QA-003). Following this procedure, a technical review would verify that the reported results are traceable, that inferences and conclusions are soundly based, and the reported work satisfies the objectives.

Key analytes in the laboratory control sample (LCS) were plotted over time to look for anomalies. In general, the plots of concentrations associated with the inductively coupled plasma (ICP) and ion chromatography (IC) analysis of solutions show recovery within limits of $80 \%$ to $120 \%$.

Additional equipment that may be used includes a thermometer, clock and balances. The thermometer for monitoring the batch-contact temperature and the timepiece are standard laboratory equipment for use as indicators only. Balances are calibrated annually by a certified contractor, QC Services, Portland, Oregon.

\section{R\&T Test Conditions}

The research and technology $(\mathrm{R} \& \mathrm{~T})$ test conditions, as defined in the Test Specifications 24590-WTP-TSP-RT-07-004, Rev. $0^{\text {(a) }}$ associated with the Test Plan TP-RPP-WTP-509, Rev 0.5, and 24590-PTF-TSP-RT-07-001, Rev. 2 (Huckaby 2008) associated with the Test Plan TP-WTP-PEP-044, Rev 0.2, are summarized in Table S.7 and S.8, respectively.

(a) PS Sundar. 2007. Simulant Testing in Support of Phase I Demonstration of the Ultrafiltration and Leaching Processes in the Integrated Test Facility. WTP Project Doc. No. 24590-WTP-TSP-RT-07-004, Rev. 0. 
Table S.7. R\&T Test Conditions for TP-RPP-WTP-509. Rev. 0.5

\begin{tabular}{|c|c|c|}
\hline \multicolumn{2}{|c|}{ List R\&T Test Conditions } & \multirow{2}{*}{$\begin{array}{l}\text { Were Test Conditions Followed? } \\
\text { Not applicable to this report. Results } \\
\text { discussed in WTP-RPT-184. }\end{array}$} \\
\hline & $\begin{array}{l}\text { Boehmite Dissolution Tests-Examine the impact of } \\
\text { aluminate, hydroxide, and other principal anions on } \\
\text { boehmite dissolution kinetics. }\end{array}$ & \\
\hline & $\begin{array}{l}\text { Boehmite Dissolution Tests-Verify the effect of } \\
\text { temperature on the dissolution of boehmite component } \\
\text { B7 and verify the effect of aluminate ion on the } \\
\text { performance of the boehmite component } \mathrm{B} 3 \text { during } \\
\text { caustic leach at temperatures lower than } 100^{\circ} \mathrm{C} \text {. }\end{array}$ & $\begin{array}{l}\text { Not applicable to this report. Results } \\
\text { discussed in WTP-RPT-184. }\end{array}$ \\
\hline & $\begin{array}{l}\text { Boehmite Dissolution Tests-Provide greater } \\
\text { discrimination on anion impact by performing tests } \\
\text { under a greater range of anion concentrations. }\end{array}$ & $\begin{array}{l}\text { Not applicable to current testing. PNNL } \\
\text { was released from this requirement by Test } \\
\text { Exception } 24590-\text { WTP-TEF-RT-07-00016. }\end{array}$ \\
\hline & $\begin{array}{l}\text { Filtration Tests-Test a base simulant under identical } \\
\text { process conditions with } 2-\mathrm{ft} \text { and } 8 \text {-ft filter elements. }\end{array}$ & $\begin{array}{l}\text { Not applicable to this report. Results } \\
\text { discussed in WTP-RPT-168. }\end{array}$ \\
\hline & $\begin{array}{l}\text { Filtration Tests-Increase the fines loading in filtration } \\
\text { test base simulant to evaluate the impact of fouling on } \\
\text { filtration performance. }\end{array}$ & $\begin{array}{l}\text { Not applicable to this report. Results } \\
\text { discussed in WTP-RPT-183. }\end{array}$ \\
\hline & $\begin{array}{l}\text { Filtration Tests- Use an } 8 \text {-ft filter element to measure } \\
\text { the filtration rate as a function of temperature up to } \\
45^{\circ} \mathrm{C} \text { for the base filtration simulant. }\end{array}$ & $\begin{array}{l}\text { Not applicable to this report. Results } \\
\text { discussed in WTP-RPT- } 168 \text {. Tests were } \\
\text { originally planned to examine temperatures } \\
\text { up to } 65^{\circ} \mathrm{C} \text {, but test exception } \\
24590 \text {-WTP-TEF-RT- } 08-00014 \text { limited the } \\
\text { upper temperature range to } 45^{\circ} \mathrm{C} \text {. }\end{array}$ \\
\hline & $\begin{array}{l}\text { Aging Tests-Perform in the } 250 \text {-gal tote and a 1-gal } \\
\text { container in the laboratory, a container in a heat-cycled } \\
\text { oven, and a baffled } 1 \text {-gal container that is mixed in the } \\
\text { laboratory. Samples are taken throughout the tests and } \\
\text { characterized by PSD, settling, rheology, and } \\
\text { centrifuged solids content to evaluate the effect of } \\
\text { aging on the behavior of the simulant. }\end{array}$ & $\begin{array}{l}\text { Not applicable to this report. Results } \\
\text { discussed in WTP-RPT-198. }\end{array}$ \\
\hline & $\begin{array}{l}\text { Chromium Simulant Leaching Tests-Perform with } \\
\text { both a caustic leach and an oxidative leach to evaluate } \\
\text { the leaching performance of the various vendor batches } \\
\text { of Cr-simulant. }\end{array}$ & $\begin{array}{l}\text { All four of the vendor Cr-simulants were } \\
\text { tested by caustic leaching with } \mathrm{NaOH} \text { at } \\
100^{\circ} \mathrm{C} \text { for } 24 \text { hours and oxidative leaching } \\
\text { using } 1 \mathrm{M} \mathrm{NaMnO}_{4} \text { at room temperature for } \\
6 \text { hours. This is described in more detail in } \\
\text { Section } 8.2 \text { along with the data being } \\
\text { presented. }\end{array}$ \\
\hline & $\begin{array}{l}\text { PEP Leaching Support Tests-Carry out with the } \\
\text { vendor-produced } 250 \text {-gal batch of the PEP simulant } \\
\text { and the vendor-produced CrOOH Test Batch } 1 \\
\text { simulant slurry. The tests are directed to determine the } \\
\text { mass loss and aluminum and chromium dissolution } \\
\text { rates during caustic leaching under varying temperature } \\
\text { processing conditions without aeration in both } \\
\text { UFP-1A/B and UFP-2A/B vessels as well as to } \\
\text { measure the effect of aeration on chromium leaching in } \\
\text { UFP-2A/B. }\end{array}$ & $\begin{array}{l}\text { The vendor-produced } 250 \text {-gallon batch of } \\
\text { PEP simulant along with the } \\
\text { vendor-produced } \mathrm{CrOOH} \text { simulant were } \\
\text { used for these tests. Five leach tests were } \\
\text { conducted at } 20-\mathrm{wt} \% \text { undissolved solids } \\
\text { (UDS), and four leach tests were conducted } \\
\text { at } 5 \text {-wt } \% \text { UDS. One of the } 20 \text {-wt } \% \text { samples } \\
\text { was aerated at } \sim 85-\mathrm{mL} / \mathrm{min} \text { during the } \\
\text { leaching time and leached at } 85^{\circ} \mathrm{C} \text {. One } \\
\text { leach test from each } \mathrm{UDS} \text { concentration was } \\
\text { conducted at } 100^{\circ} \mathrm{C}, 95^{\circ} \mathrm{C}, 85^{\circ} \mathrm{C} \text {, and } 80^{\circ} \mathrm{C} \text {. } \\
\text { The vessels were heated over } 6 \text { hours to the }\end{array}$ \\
\hline
\end{tabular}


Table S.7. R\&T Test Conditions for TP-RPP-WTP-509. Rev. 0.5

\begin{tabular}{|c|c|}
\hline List R\&T Test Conditions & Were Test Conditions Followed? \\
\hline & $\begin{array}{l}\text { leach temperature and held at the leach } \\
\text { temperature for } 24 \text { hours. Samples were } \\
\text { taken at } 1,2,4,8,12,16,20 \text {, and } 24 \text { hours. } \\
\text { This is described in more detail in } \\
\text { Section } 5.0 \text { along with the data being } \\
\text { presented. }\end{array}$ \\
\hline $\begin{array}{l}\text { 10) PEP Leaching Support Tests-Perform using a } \\
\text { vendor-produced } 250 \text {-gal batch of the PEP simulant. } \\
\text { The tests are directed to measure the extent of } \\
\text { boehmite conversion expected under leaching } \\
\text { conditions during the planned testing in the PEP. }\end{array}$ & $\begin{array}{l}\text { The first three tests were performed by } \\
\text { centrifuging } 5 \text {-wt } \% \text { UDS PEP simulant to } \\
\text { remove enough supernate to concentrate it to } \\
20 \text {-wt } \% \text { UDS. Then the } 20 \text {-wt } \% \text { UDS PEP } \\
\text { simulant was placed into the test vessel with } \\
\text { the correct amount of deionized (DI) water } \\
\text { and NaOH. The fifth test was performed in } \\
\text { the same manner as the first three, except it } \\
\text { was not centrifuged, and the original } 5 \text {-wt } \% \\
\text { UDS PEP simulant was used. The fourth test } \\
\text { was performed by placing the original } \\
5 \text {-wt } \% \text { UDS PEP simulant into the test } \\
\text { vessel with the correct amount of DI water } \\
\text { and NaOH and heating it to } 60^{\circ} \mathrm{C} \text {, holding } \\
\text { for } 8 \text { hours, and then cooling. After the } \\
\text { simulant was cooled, it was centrifuged, and } \\
\text { supernate was removed to concentrate it to } \\
20 \text {-wt } \% \text { UDS. Then the } 20 \text {-wt } \% \text { UDS PEP } \\
\text { simulant was placed into the test vessel with } \\
\text { the correct amount of DI water and NaOH. } \\
\text { The vessels were then heated to the target } \\
\text { leach temperature and an initial sample was } \\
\text { taken. Samples were also taken at } 1,2,4,8 \text {, } \\
12,24 \text {, and } 30 \text { hours. This is described in } \\
\text { more detail in Section } 5.0 \text { along with the } \\
\text { data being presented. }\end{array}$ \\
\hline
\end{tabular}


Table S.8. R\&T Test Conditions for TP-WTP-PEP-044, Rev. 0.2

\begin{tabular}{|c|c|c|}
\hline \multicolumn{2}{|r|}{ List R\&T Test Conditions } & \multirow{2}{*}{$\begin{array}{l}\text { Were Test Conditions Followed? } \\
\text { Not applicable to this report. It is addressed } \\
\text { in reports WTP-RPT-185 and } \\
\text { WTP-RPT-203. }\end{array}$} \\
\hline 1) & $\begin{array}{l}\text { A total of four tests are anticipated. Two of these tests } \\
\text { will be conducted entirely with a low-solids } \\
\text { concentration simulant (approximately } 5 \text {-wt } \% \text { ), and the } \\
\text { others will be conducted as the simulant is increased to } \\
\text { a high-solids concentration (target of } 20 \text {-wt } \% \text {, which is } \\
\text { PEP's dewatered high-solids target) through } \\
\text { dewatering. The first low-solids and dewatering test } \\
\text { will be performed with as-received simulant as will the } \\
\text { first dewater test. The other tests will be performed } \\
\text { with leached materials. These conditions are outlined } \\
\text { in Table } 5.1 \text {. These test conditions are identical to } \\
\text { those that will be used in PEP testing (using samples } \\
\text { pulled from PEP). }\end{array}$ & \\
\hline 2) & $\begin{array}{l}\text { These caustic leaching tests will be conducted with } \\
\text { slurry samples collected from the PEP leaching vessels } \\
\text { just before steam heating is initiated in the PEP after } \\
\text { the NaOH has been added. A total of four tests is } \\
\text { anticipated. Two of these tests will emulate } \\
\text { UFP-VSL-T01A/B (UFP-1) caustic leaching } \\
\text { conditions, and two tests will emulate UFP-VSL-T02A } \\
\text { (UFP-2) conditions. Test conditions will be specified } \\
\text { by WTP to match the PEP. }\end{array}$ & $\begin{array}{l}\text { These tests were performed using samples } \\
\text { from PEP taken both just before and just } \\
\text { after the caustic solution was added. } \\
\text { According to instructions from WTP, the } \\
\text { specified amount of caustic, DI water and } \\
\text { sample was added and leached at the } \\
\text { specified temperature for } 24 \text { hours after the } \\
\text { specified heating time. Samples were taken } \\
\text { at } 10^{\circ} \mathrm{C} \text { below specified temperature and } \\
\text { then } 1 \text { and } 2 \text { hours after temperature was } \\
\text { reached and every } 2 \text { hours over the } 24 \text {-hour } \\
\text { period at } 98^{\circ} \mathrm{C} \text { for the Functional tests, Tests } \\
\text { A and } \mathrm{B} \text {, and } 85^{\circ} \mathrm{C} \text { for Test D. These results } \\
\text { are presented in Section } 6.0 \text {. }\end{array}$ \\
\hline 3) & $\begin{array}{l}\text { Stirred-reactor oxidative leaching tests will be } \\
\text { conducted with slurry samples collected from UFP-2 in } \\
\text { the PEP just before permanganate is added in the PEP. } \\
\text { A total of four tests is anticipated, one for each of the } \\
\text { four integrated process tests. }\end{array}$ & $\begin{array}{l}\text { These tests were performed using samples } \\
\text { from PEP just before the permanganate was } \\
\text { to be added. According to instructions from } \\
\text { WTP, the specified amount of } 1 \mathrm{M} \mathrm{NaMnO}_{4} \\
\text { was added to the specified amount of } \\
\text { sample. The samples were then leached at } \\
\text { room temperature for } 8 \text { hours with a sample } \\
\text { taken every hour. These results are } \\
\text { presented in Section } 6.5 \text {. }\end{array}$ \\
\hline 4) & $\begin{array}{l}\text { Several permeate samples will be collected throughout } \\
\text { the testing from the PEP, maintained for } 10 \text { days at } \\
\text { room temperature, and then examined for precipitates. } \\
\text { Some will be kept separate, and others will be mixed to } \\
\text { provide various conditions. }\end{array}$ & $\begin{array}{l}\text { This was performed on PEP Test A } \\
\text { post-caustic wash solutions by allowing } \\
\text { several samples to sit for } 10 \text { days; several } \\
\text { other samples were blended and then } \\
\text { allowed to sit for } 10 \text { days also. All of the } \\
\text { samples showed significant precipitation at } \\
\text { the end of the } 10 \text { days that appeared to be a } \\
\text { mixture of oxalate and phosphate. These } \\
\text { results are presented in Section } 7.0 \text {. }\end{array}$ \\
\hline
\end{tabular}




\section{Simulant Use}

Actual Hanford tank waste cannot be used in the PEP because of safety, cost, and volume concerns. To address the need to demonstrate separation and leaching processes at PEP, PNNL developed a waste simulant that mimics the chemical, leaching, and ultrafiltration behaviors of actual tank waste under Test Plan TP-RPP-WTP-469, Rev. 0. A simulant formulation developed under TP-RPP-WTP-469 was used for simulant tests described in the controlling test plans, TP-RPP-WTP-509 and TP-WTP-PEP-044.

PEP process testing was performed with a nonradioactive aqueous slurry of simulant waste chemicals and solids. The simulant composition and make-up recipe were provided by WTP as documented in Simulant Recommendation for Phase 1 Testing in the Pretreatment Engineering Platform. ${ }^{\text {(a) }}$ Aqueous chemical concentrations were within ranges expected for waste feeds to the PTF except for the hydroxide, oxalate and phosphate anions. The hydroxide concentration was near one standard deviation from the average concentration expected in the feeds to the plant. The oxalate and phosphate components were at or near their respective solubility limits. The solids components and blend were selected to obtain targeted solids mass loss (aluminum and chromium leaching and oxalate washing) and treatment time. The simulant was not selected to represent any particular Hanford tank waste type.

The simulant was blended from the components listed below. The basis for selecting the individual components and the comparison to actual waste behavior are provided where applicable in the indicated references.

- Boehmite (for Al) (Russell et al. 2009a)

- Gibbsite (for Al) (Russell et al. 2009b)

- Chromium oxyhydroxide (CrOOH) slurry (Rapko et al. 2007)

- Sodium oxalate

- Filtration simulant (Russell et al. 2009c)

- Supernate.

Because the high-temperature caustic leaching process was found to dissolve significant amounts of the $\mathrm{CrOOH}$ solids, a separate chromium solids simulant was prepared and added to the PEP process after post-caustic-leach washing (a non-prototypic addition) in Integrated Tests A and B. In Test D, the chromium solids component of the simulant was added to the feed to demonstrate the PTF permanganate addition strategy.

Simulant was procured from NOAH Technologies Corporation (San Antonio, TX). Samples of each simulant batch were characterized to make certain that chemical and physical property requirements were met. Batches of the simulant were procured as follows:

- A 15-gallon trial batch of the blended simulant for laboratory testing to demonstrate the efficacy of the simulant fabrication procedure.

- A 250-gallon scale-up batch of the blended simulant to demonstrate scale-up of the simulant fabrication procedure to an intermediate scale.

(a) PS Sundar. 2008. Simulant Recommendation for Phase 1 Testing in the Pretreatment Engineering Platform. 24590-PTF-RT-08-006, Rev 0, Bechtel National, Inc., Richland, Washington. 
- Batches 0, 1, and 2, each nominally 3500 gallons, of blended simulant for the Shakedown/Functional Tests and Integrated Tests A and B. These batches did not contain the $\mathrm{CrOOH}$ component.

- Batch 3, nominally 1200 gal, for Integrated Test D. This batch contained the CrOOH solids component.

- The CrOOH solids slurry for the Shakedown/Functional Test and Tests A and B was obtained in two separate batches containing nominally 18 and $36 \mathrm{~kg}$ of $\mathrm{Cr}$ as $\mathrm{CrOOH}$.

\section{Discrepancies and Follow-on Tests}

None. 


\subsection{Background}

Pacific Northwest National Laboratory (PNNL) has been tasked by Bechtel National Inc. (BNI) on the River Protection Project-Hanford Tank Waste Treatment and Immobilization Plant (RPP-WTP) project to perform research and development activities to resolve technical issues identified for the Pretreatment Facility (PTF). The Pretreatment Engineering Platform (PEP) was designed, constructed, and operated as part of a plan to respond to issue M12, "Undemonstrated Leaching Processes," of the External Flowsheet Review Team (EFRT) issue response plan. ${ }^{\text {(a) }}$ The PEP is a $1 / 4.5$-scale test platform designed to simulate the WTP pretreatment caustic leaching, oxidative leaching, ultrafiltration solids concentration, and slurry washing processes. The PEP replicates the WTP leaching processes using prototypic equipment and control strategies. A simplified flow diagram of the PEP system is shown in Figure 1.1.

Two operating scenarios are currently being evaluated for the ultrafiltration process (UFP) and leaching operations. The first scenario has caustic leaching performed in the UFP-2 ultrafiltration feed vessels (i.e., vessel UFP-VSL-T02A in the PEP and vessels UFP-VSL-00002A and B in the WTP PTF). The second scenario has caustic leaching conducted in the UFP-1 ultrafiltration feed preparation vessels (i.e., vessels UFP-VSL-T01A and B in the PEP and vessels UFP-VSL-00001A and B in the WTP PTF).

In both scenarios, 19-M sodium hydroxide solution ( $\mathrm{NaOH}$, caustic) is added to the waste slurry in the vessels to leach solid aluminum compounds (e.g., gibbsite, boehmite). Caustic addition is followed by a heating step that uses direct injection of steam to accelerate the leach process. Following the caustic leach, the vessel contents are cooled using vessel cooling jackets and/or external heat exchangers. The main difference between the two scenarios is that for leaching in UFP-VSL-T01A and B, the 19-M NaOH is added to unconcentrated waste slurry (3- to 8 -wt $\%$ solids), while for leaching in UFP-VSL-T02A, the slurry is concentrated to nominally 20 -wt $\%$ solids using cross-flow ultrafiltration before adding caustic.

The PEP testing program was conducted under Test Plan TP-RPP-WTP-506 $6^{(\mathrm{b})}$ using a waste simulant, which was developed in response to Task 5 from the M-12 EFRT issue response plan. The testing included the following tests with simulated Hanford tank waste:

- Shakedown/Functional Testing: Tested process operations (e.g., slurry transfers, steam heating of the vessels and the accumulation of condensate, filter backpulsing, and flushing), process controls (e.g., transmembrane pressure and axial flow velocity in the filter loop), and certain test functions (e.g., in-line slurry sampling accuracy and precision).

- Integrated Test A: Demonstrated integrated processing when caustic leaching $\left(98^{\circ} \mathrm{C}\right)$ is performed in UFP-VSL-00001A/B with the Cr simulant component added after the post-caustic-leach washing step.

- Integrated Test B: Demonstrated integrated processing when the caustic leaching $\left(98^{\circ} \mathrm{C}\right)$ is performed in UFP-VSL-00002A with the Cr simulant component added after the post-caustic-leach washing step.

(a) SM Barnes and R Voke. 2006. "Issue Response Plan for Implementation of External Flowsheet Review Team (EFRT) Recommendations - M12: Undemonstrated Leaching Process.” 24590-WTP-PL-ENG-06-0024 Rev. 0.

(b) GB Josephson, OP Bredt, JK Young, and DE Kurath. 2008. Pretreatment Engineering Platform (PEP) Testing (Phase I). TP-RPP-WTP-506, Rev. 0.3, Pacific Northwest National Laboratory, Richland, Washington. 
- Integrated Test D: Demonstrated integrated processing when the caustic leaching is performed at a lower temperature $\left(85^{\circ} \mathrm{C}\right)$ in UFP-VSL-00002A and with the $\mathrm{Cr}$ simulant component added to the initial batch of simulant.

Integrated Test $\mathrm{C}$ was deleted from the scope of the testing (ICN-TP-RPP-WTP-506_R0.2).

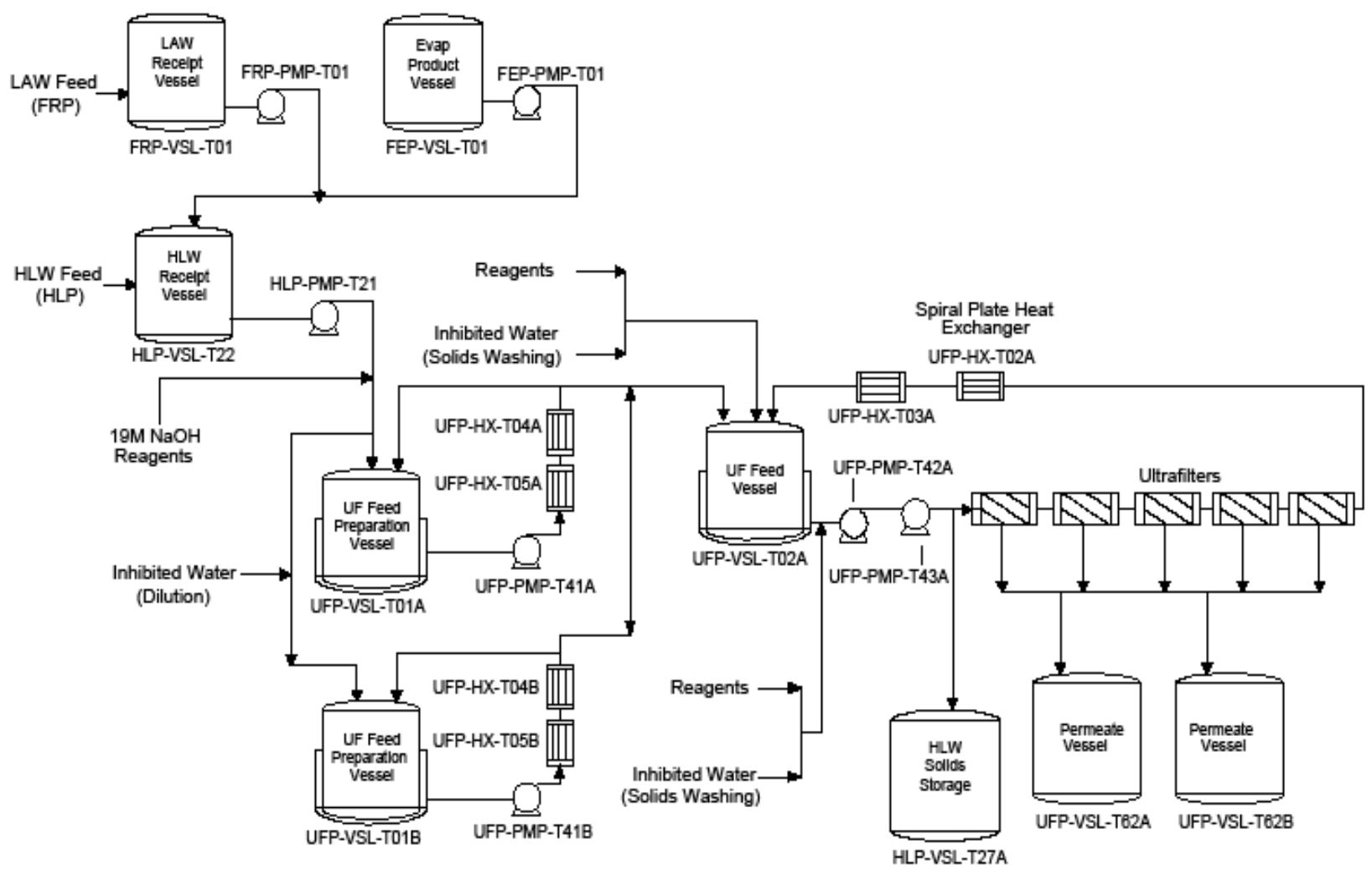

Figure 1.1. PEP Simplified Flow Diagram

Caustic-leaching data are needed on the various types of wastes to be processed through the WTP to support the plant design. The data needed include 1) removal of key HLW sludge components (e.g., Al, $\mathrm{Cr}, \mathrm{P}$, and $\mathrm{S}$ ) as a function of caustic concentration, temperature, and time, 2) the behavior of radionuclides during the leaching process, 3) particle-size distribution (PSD), and 4) identification of the chemical and mineral forms of important sludge components (e.g., Al, Cr, and P) in the sludge solids. These new data will support the development of various waste simulants for scaled process demonstrations.

Aluminum in the wastes is believed to be present in the two most common mineralogical phases: gibbsite (monoclinic $\mathrm{Al}(\mathrm{OH})_{3}$ ) and boehmite (orthorhombic $\mathrm{AlOOH}$ ). Other phases present include bayorite, dawsonite, alumina silicates, and amorphous aluminum hydroxide. The dissolution rates of the two primary mineralogical phases are considerably different. Therefore, the leaching kinetics will depend on the relative amounts of these phases in the waste as well as particle size, crystal habit (i.e., size and shape), operating temperature, hydroxide activity, aluminum solubility limits, particle Reynolds number associated with the mixing system, etc. While there may be other phases of aluminum compounds in the 
waste solids, they are present in relatively small amounts and therefore are considered less significant to the caustic leaching for removing aluminum from the high-level waste (HLW).

This report addresses several testing objectives. The experimental methods used in this testing are described in Section 3. The first testing objective provided input to a boehmite dissolution model and is described in Section 4 of this report with the model being described in more detail in Appendix A. The objectives of the next tests were to determine the effect of temperature on the solids mass loss during caustic leaching, the reaction rate constant for the boehmite dissolution, and the effect of aeration in enhancing the chromium dissolution during caustic leaching using the PEP simulant. This is described in Section 5. Section 6 describes the tests that were performed in parallel with the PEP tests to support the development of scaling factors for caustic and oxidative leaching. A study was performed to determine if precipitate formed in the wash solution after the caustic leach in the PEP and is described in Section 7. The final task was to examine the leaching characteristics of different chromium compounds under different conditions to determine the best one to use in further testing. This task is described in Section 8.

Table 1.1 summarizes the testing objectives and sample identification nomenclature covered in this report.

Table 1.1. Testing Objectives and Sample Identification Nomenclature

\begin{tabular}{|c|c|c|c|}
\hline Section & Test Objectives & Sample Types & Sample Identification Nomenclature \\
\hline 4 & $\begin{array}{l}\text { Develop boehmite } \\
\text { dissolution model as a } \\
\text { function of test time. }\end{array}$ & $\begin{array}{l}\text { Filtrate samples from } \\
\text { caustic boehmite } \\
\text { leaching tests. }\end{array}$ & $\begin{array}{l}\text { B-AL1 to B-AL20. } \\
\text { B-AL1a to B-AL10a. }\end{array}$ \\
\hline 5 & $\begin{array}{l}\text { Determine the effect of } \\
\text { temperature on boehmite } \\
\text { leaching coefficient and the } \\
\text { effect of aeration on } \\
\text { chromium leaching. }\end{array}$ & $\begin{array}{l}\text { Slurry and supernate } \\
\text { taken as a function of } \\
\text { time and temperature. }\end{array}$ & $\begin{array}{l}\text { PST-1 to PST-9. } \\
\text { PST2-1 to PST2-7. } \\
\text { INS = initial slurry. } \\
\text { FIS = initial washed slurry before heating. } \\
\text { FWS = final washed slurry after cooled. }\end{array}$ \\
\hline 6 & Determine scale factors betw & en PEP and laboratory tests & \\
\hline 6.1 & $\begin{array}{l}\text { Make direct comparison } \\
\text { with the PEP Functional } \\
\text { Caustic Leach Tests. }\end{array}$ & $\begin{array}{l}\text { Slurry and supernate } \\
\text { taken as a function of } \\
\text { time and temperature. }\end{array}$ & $\begin{array}{l}\text { PL-1 to PL-4. } \\
\text { LAB CL = Lab Caustic Leach. } \\
\text { IS_SL = initial washed slurry. } \\
\text { FS } 1 \text { to } 3 \text { _SL = final washed slurry sample } 1 \\
\text { to } 3 \text {. } \\
\text { A_LAB_CL_1_IS_SL, etc. }=\text {. } \\
\text { in Tables } 6.5 \text { thru } 6.8 \text {, and } 6.13, \mathrm{~A}=\mathrm{PL} \text {. } \\
\text { IN_SL = initial slurry. } \\
\text { FS = final washed slurry. } \\
\text { SUP = supernate. }\end{array}$ \\
\hline 6.2 & $\begin{array}{l}\text { Make laboratory } \\
\text { comparisons to PEP Test A } \\
\text { Caustic Leach Tests. }\end{array}$ & $\begin{array}{l}\text { Slurry and supernate } \\
\text { taken as a function of } \\
\text { time and temperature. }\end{array}$ & $\begin{array}{l}\text { A_LAB_CL = PEP Parallel Test A } \\
\text { Laboratory Caustic Leach. } \\
\text { SL = slurry. } \\
\text { ISW = initial washed slurry. } \\
\text { IS = initial unwashed slurry. } \\
\text { FS1 through FS3 = final washed slurry. } \\
\text { FS4 = final unwashed slurry. }\end{array}$ \\
\hline
\end{tabular}




\begin{tabular}{|c|c|c|c|}
\hline 6.3 & $\begin{array}{l}\text { Make laboratory } \\
\text { comparisons to PEP Test B } \\
\text { Caustic Leach Tests. }\end{array}$ & $\begin{array}{l}\text { Slurry and supernate } \\
\text { taken as a function of } \\
\text { time and temperature. }\end{array}$ & $\begin{array}{l}\text { B_LAB_CL }=\text { PEP Parallel Test B Laboratory } \\
\text { Caustic Leach. } \\
\text { SL = slurry. } \\
\text { ISW = initial washed slurry. } \\
\text { IS = initial unwashed slurry. } \\
\text { FS1 and FS2 = final washed slurry. } \\
\text { FS3 = final unwashed slurry. }\end{array}$ \\
\hline 6.4 & $\begin{array}{l}\text { Make laboratory } \\
\text { comparisons to PEP Test D } \\
\text { Caustic Leach Tests. }\end{array}$ & $\begin{array}{l}\text { Slurry and supernate } \\
\text { taken as a function of } \\
\text { time and temperature. }\end{array}$ & $\begin{array}{l}\text { D_LAB_CL }=\text { PEP Parallel Test D } \\
\text { Laboratory Caustic Leach. } \\
\text { SL = slurry. } \\
\text { ISW = initial washed slurry. } \\
\text { IS = initial unwashed slurry. } \\
\text { FSW = final washed slurry. } \\
\text { FS = final unwashed slurry. }\end{array}$ \\
\hline 6.5 & $\begin{array}{l}\text { Make laboratory } \\
\text { comparisons to PEP } \\
\text { Oxidative Leach Tests. }\end{array}$ & $\begin{array}{l}\text { Slurry and supernate } \\
\text { taken as a function of } \\
\text { time and temperature. }\end{array}$ & $\begin{array}{l}\text { A_LAB_OL }=\text { PEP Parallel Test A } \\
\text { Laboratory Oxidative Leach. } \\
\text { B_LAB_OL }=\text { PEP Parallel Test B Laboratory } \\
\text { D_LAB_OL }=\text { PEP Parallel Test D } \\
\text { Laboratory Oxidative Leach. } \\
\text { ORG_SUP }=\text { original supernate before } \mathrm{MnO}_{4}^{-} \\
\text {addition. } \\
\text { INA_SUP = initial supernate after } \mathrm{MnO}_{4}^{-} \\
\text {addition. } \\
\mathrm{SL}=\text { slurry. } \\
\text { IS = initial slurry after } \mathrm{MnO}_{4}^{-} \text {addition. } \\
\text { ISW = initial washed slurry after } \mathrm{MnO}_{4}^{-} \\
\text {addition. } \\
\text { OSW = initial washed slurry before } \mathrm{MnO}_{4}^{-} \\
\text {addition. } \\
\text { OS = initial slurry before } \mathrm{MnO}_{4}^{-} \text {addition. } \\
\text { FWS = final washed slurry. } \\
\text { FS = final slurry. }\end{array}$ \\
\hline 8 & $\begin{array}{l}\text { Test leaching properties of } \\
\text { different chromium } \\
\text { compounds under different } \\
\text { conditions. }\end{array}$ & $\begin{array}{l}\text { Supernate taken as a } \\
\text { function of time and } \\
\text { temperature. }\end{array}$ & $\begin{array}{l}\text { CL-C1 to CL-C7, OL-C1 to OL-C7. } \\
\text { FMC1 to FMC5, FMO1 to FMO5. } \\
\text { CL-CO1 to CL-CO6, OL-CO1 to OL-CO6. } \\
\text { CLI1 to CLI5. } \\
\text { CL = caustic leach. } \\
\text { OL = oxidative leach. } \\
\text { FMC = full-matrix caustic leach. } \\
\text { FMO = full-matrix oxidative leach. }\end{array}$ \\
\hline
\end{tabular}




\subsection{Quality Assurance}

The PNNL QA program is based upon the requirements as defined in the U.S. Department of Energy (DOE) Order 414.1C, Quality Assurance, and 10 CFR 830, Energy/Nuclear Safety Management, Subpart A-Quality Assurance Requirements (a.k.a. the Quality Rule). PNNL has chosen to implement the following consensus standards in a graded approach:

- ASME NQA-1-2000, Quality Assurance Requirements for Nuclear Facility Applications, Part 1, Requirements for Quality Assurance Programs for Nuclear Facilities.

- ASME NQA-1-2000, Part II, Subpart 2.7, Quality Assurance Requirements for Computer Software for Nuclear Facility Applications.

- ASME NQA-1-2000, Part IV, Subpart 4.2, Graded Approach Application of Quality Assurance Requirements for Research and Development.

The procedures necessary to implement the requirements are documented through PNNL's Standards-Based Management System (SBMS).

PNNL implements the RPP-WTP quality requirements by performing work in accordance with the River Protection Project-Hanford Tank Waste Treatment and Immobilization Plant Support Program (RPP-WTP) Quality Assurance Plan (RPP-WTP-QA-001, QAP). Work was performed to the quality requirements of NQA-1-1989 Part I, Basic and Supplementary Requirements, NQA-2a-1990, Part 2.7, and DOE/RW-0333P, Rev 13, Quality Assurance Requirements and Descriptions (QARD), as applicable. These quality requirements are implemented through the River Protection Project - Waste Treatment Plant Support Program (RPP-WTP) Quality Assurance Manual (RPP-WTP-QA-003, QAM). The requirements of DOE/RW-0333P Rev 13, Quality Assurance Requirements and Descriptions (QARD) and 10 CFR 830 Subpart A were not required for this work.

The RPP-WTP addresses internal verification and validation activities by conducting an independent technical review of the final data report in accordance with RPP-WTP's procedure QA-RPP-WTP-604. This review procedure is part of PNNL's RPP-WTP Quality Assurance Manual (RPP-WTP-QA-003). Following this procedure, a technical review would verify that the reported results are traceable, that inferences and conclusions are soundly based, and the reported work satisfies the objectives.

Key analytes in the laboratory control sample (LCS) were plotted over time to look for anomalies. In general, the plots of concentrations associated with the inductively coupled plasma (ICP) and ion chromatography (IC) analysis of solutions show recovery within limits of $80 \%$ to $120 \%$.

Additional equipment that may be used includes a thermometer, clock, and balances. The thermometer for monitoring the batch-contact temperature and the timepiece are standard laboratory equipment for use as indicators only. Balances are calibrated annually by a certified contractor, QC Services, Portland, Oregon. 



\subsection{Experimental Methods}

The caustic leaching tests were set up as shown in Figure 3.1 using 1-wt\% solids or PEP slurry in 800 to 900 grams of total solution (solids + liquid) that was heated to the appropriate temperature while stirring in a 1-liter polymethyl pentene (PMP) reaction vessel. The material to be leached is added to the reaction vessel through the sample port while stirring after the leaching solution has reached leaching temperature, which starts the clock for the test when powders are being leached. When slurries are being leached, they are added at the beginning and heated along with the leach solution. The test solution is sampled while stirring at the specified sampling hours. Each supernatant sample consists of 5-mL slurry, which is filtered through a $0.45-\mu \mathrm{m}$ syringe filter to produce supernatant.

Five reaction vessel systems were typically run in parallel, allowing five tests to be performed at once. Each reaction vessel is a straight-side wide-mouth jar with an inside height of $116 \mathrm{~mm}$ and an inside diameter of $110 \mathrm{~mm}$. It has three PMP baffles, $92 \mathrm{~mm}$ long, $5 \mathrm{~mm}$ thick, and $19 \mathrm{~mm}$ wide, evenly spaced around the vessel. Figure 3.2 shows a picture of the testing vessel. A stainless steel stir shaft ( $8 \mathrm{~mm}$ in diameter and $305 \mathrm{~mm}$ long) with a $95 \mathrm{~mm}$ wide blade welded on the bottom, is used to stir the vessel contents as shown in Figure 3.3. The blade is $13 \mathrm{~mm}$ tall, and each blade has a $45^{\circ}$ pitch and pushes the fluid down while stirring. Stirring was consistently performed at $150 \mathrm{rpm}$ for these tests, which provides complete mixing. A heating jacket is wrapped around the vessel to maintain the test mixture at a constant temperature throughout the test. The temperature is measured with a calibrated thermocouple (TC) and controlled with a calibrated temperature controller. Aeration was performed by passing building air through a rotometer and into a metal tube that had been placed near the bottom of the reaction vessel.

All tests were monitored for evaporation by comparing the sodium levels in the tests because the sodium level should not have been changing. When aeration was not performed, the tests were so short and the vessels were so tightly sealed that evaporation was not an issue. However, when aeration was performed, a level in the reaction vessel was maintained by marking the initial solution level and maintaining that by adding deionized water periodically.

Some slurry samples were also analyzed by the same methods as the supernate samples after they were washed three times in $0.01 \mathrm{M} \mathrm{NaOH}$ before being submitted for chemical analysis. The samples were chemically and physically analyzed by inductively coupled plasma-optical emission spectrometry (ICP-OES), inductively coupled plasma-atomic emission spectrometry (ICP-AES), ion chromatography (IC), potentiometric titration for free $\mathrm{OH}$, gravimetric measurement for density, and undissolved solids (UDS). However, not all samples were analyzed by all methods. The majority of the analyses were performed at Southwest Research Institute, Inc. (SWRI) with some of the analyses performed by PNNL. All chemical analyses were performed to the requirements of the Hanford Analytical Services Quality Assurance Requirements Documents, DOE/RL-96-68, HASQARD. 


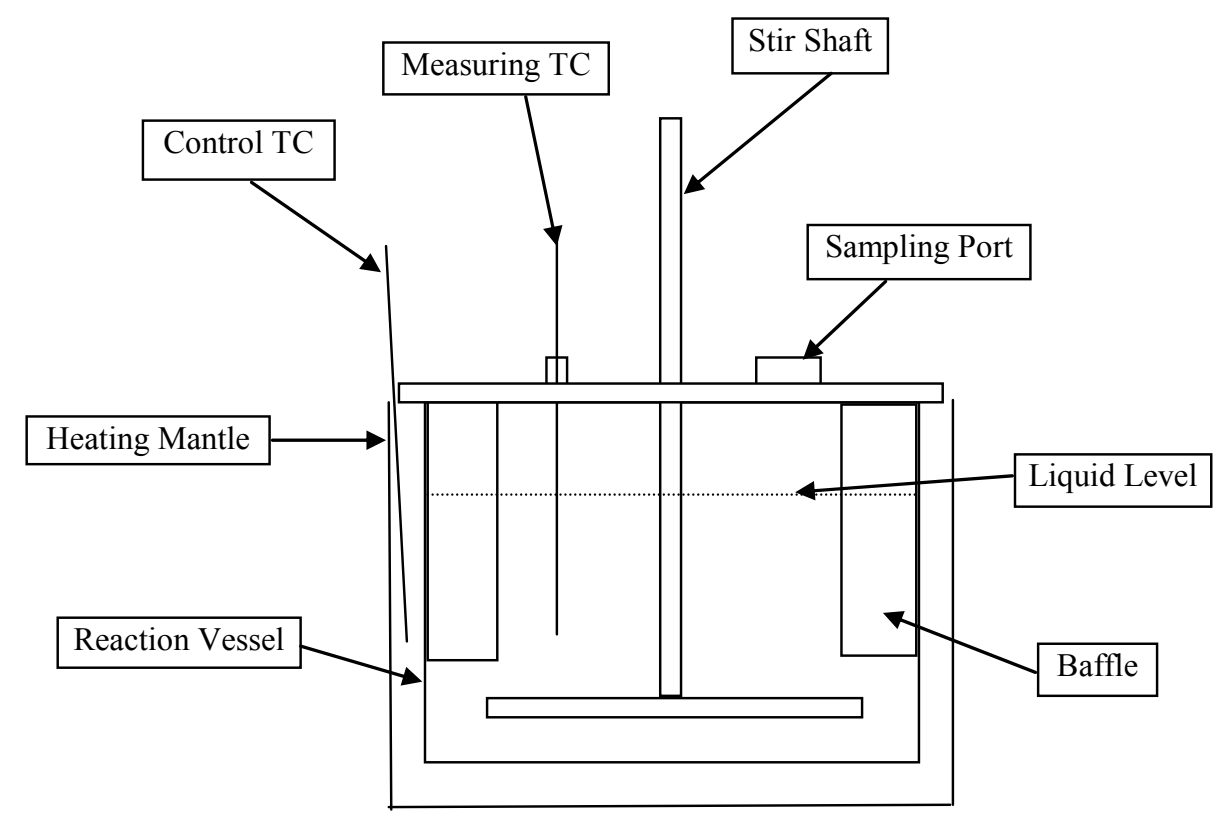

Figure 3.1. Schematic Drawing of the Caustic Leaching Test Setup

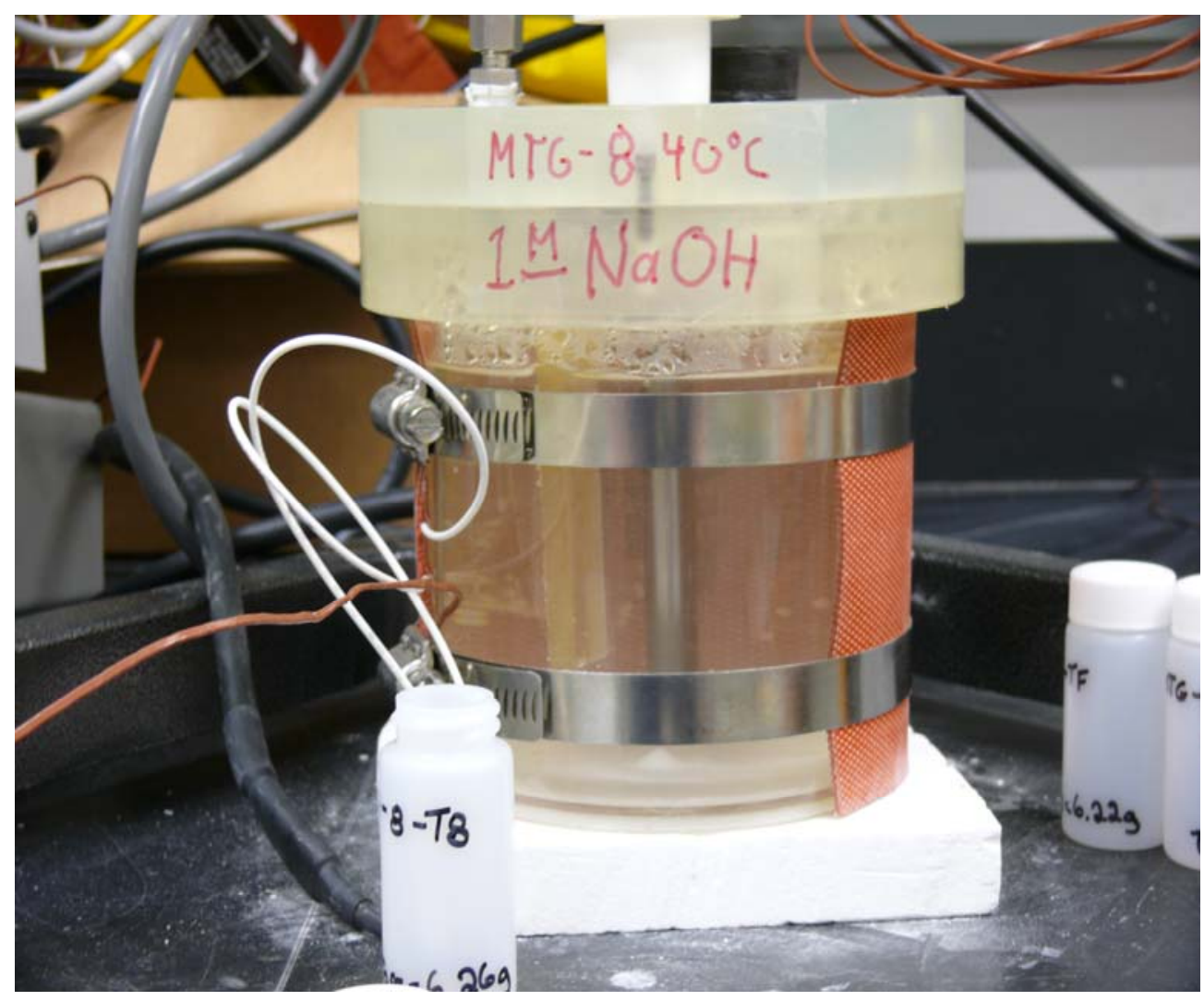

Figure 3.2. Simulant Leaching Vessel 


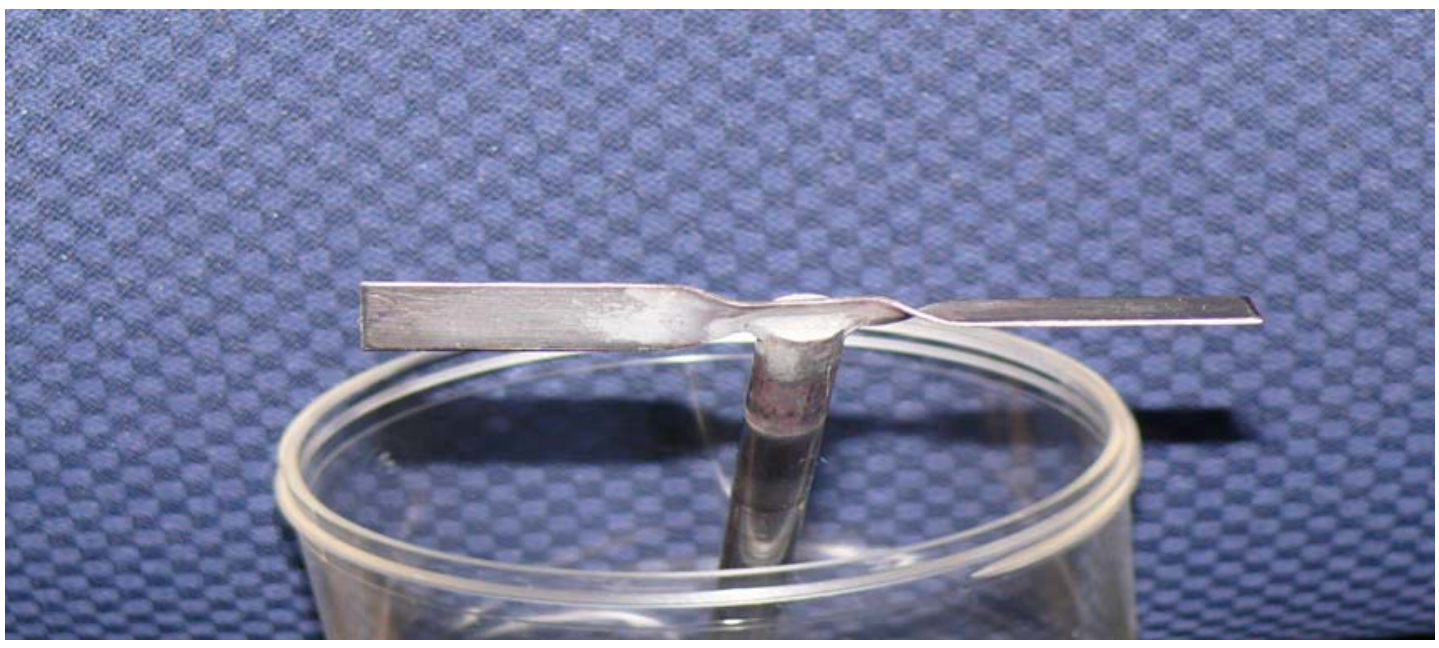

Figure 3.3. Simulant Leaching Vessel Stir Assembly 



\subsection{Model Input Boehmite Testing}

The objective of these tests was to determine the effect of aluminate ion concentration and temperature on the rate of boehmite leaching. These tests were specified in

ICN-TP-RPP-WTP-509_R0.5. Table 4.1 and Table 4.2 show the test matrices that were used for these tests. Boehmite type B3 indicates that Nabaltec APYRAL AOH20 was used, and boehmite type B7 indicates that Nabaltec APYRAL AOH180E was used. Boehmite type B3 was used in the PEP simulant for all of the PEP testing. The first 10 tests in Table 4.1 were designed to determine the impact of aluminate ion concentration on boehmite leaching. Tests 11 through 16 were designed to determine the effect of temperature on the boehmite dissolution, and tests 17 through 20 were designed to verify the effect of aluminate ion at lower reaction temperatures in the development of the boehmite dissolution rate equation.

The tests in Table 4.2 were also designed to determine the boehmite dissolution rate incorporating the impact of aluminate ion. These tests varied the amount of gibbsite present and determined the effect that it had on the boehmite dissolution. Data from tests in both Table 4.1 and Table 4.2 were all used in the boehmite dissolution rate equation development.

Table 4.1. Initial Set of Boehmite Model Input Tests

\begin{tabular}{ccccccc}
\hline Test ID & $\begin{array}{c}\text { Boehmite } \\
\text { Type }\end{array}$ & $\begin{array}{c}\text { Boehmite } \\
(\mathrm{g})\end{array}$ & $\begin{array}{c}\text { Gibbsite } \\
(\mathrm{g})\end{array}$ & $\begin{array}{c}5 \mathrm{M} \\
\mathrm{NaOH}(\mathrm{g})\end{array}$ & $\begin{array}{c}\text { Temperature } \\
\left({ }^{\circ} \mathrm{C}\right)\end{array}$ & $\begin{array}{c}\text { Mixing } \\
\text { Speed (rpm) }\end{array}$ \\
\hline B-AL1 & B3 & 36.00 & 0.00 & 900.00 & 100 & 120 \\
B-AL2 & B3 & 36.00 & 5.06 & 900.00 & 100 & 120 \\
B-AL3 & B3 & 36.00 & 10.13 & 900.01 & 100 & 120 \\
B-AL4 & B3 & 36.00 & 15.20 & 899.99 & 100 & 120 \\
B-AL5 & B3 & 36.00 & 20.26 & 900.01 & 100 & 120 \\
B-AL6 & B3 & 36.00 & 25.33 & 900.01 & 100 & 120 \\
B-AL7 & B3 & 36.00 & 30.39 & 900.00 & 100 & 120 \\
B-AL8 & B3 & 36.00 & 40.52 & 900.02 & 100 & 120 \\
B-AL9 & B3 & 36.00 & 50.65 & 900.00 & 100 & 120 \\
B-AL10 & B3 & 36.00 & 60.78 & 900.01 & 100 & 120 \\
B-AL11 & B3 & 36.00 & 0.00 & 900.02 & 90 & 120 \\
B-AL12 & B3 & 36.01 & 0.00 & 900.00 & 85 & 120 \\
B-AL13 & B3 & 36.01 & 0.00 & 900.00 & 80 & 120 \\
B-AL14 & B7 & 36.00 & 0.00 & 900.01 & 90 & 120 \\
B-AL15 & B7 & 36.00 & 0.00 & 899.98 & 85 & 120 \\
B-AL16 & B7 & 36.01 & 0.00 & 900.00 & 80 & 120 \\
\hline B-AL17 & B3 & 36.00 & 10.38 & 900.01 & 85 & 120 \\
B-AL18 & B3 & 36.01 & 20.76 & 900.00 & 85 & 120 \\
B-AL19 & B3 & 36.01 & 31.14 & 900.01 & 85 & 120 \\
B-AL20 & B3 & 36.01 & 41.52 & 900.00 & 85 & 120 \\
\hline
\end{tabular}


Table 4.2. Second Set of Boehmite Model Input Tests

\begin{tabular}{ccccccc}
\hline Test ID & $\begin{array}{c}\text { Boehmite } \\
\text { Type }\end{array}$ & $\begin{array}{c}\text { Boehmite } \\
(\mathrm{g})\end{array}$ & $\begin{array}{c}\text { Gibbsite } \\
(\mathrm{g})\end{array}$ & $\begin{array}{c}5 \mathrm{M} \\
\mathrm{NaOH}(\mathrm{g})\end{array}$ & $\begin{array}{c}\text { Temperature } \\
\left({ }^{\circ} \mathrm{C}\right)\end{array}$ & $\begin{array}{c}\text { Mixing } \\
\text { Speed }(\mathrm{rpm})\end{array}$ \\
\hline B-AL1a & B3 & 36.00 & 0.00 & 900.01 & 100 & 120 \\
B-AL2a & B3 & 36.00 & 6.15 & 900.00 & 100 & 120 \\
B-AL3a & B3 & 36.00 & 12.31 & 900.01 & 100 & 120 \\
B-AL4a & B3 & 35.99 & 18.46 & 900.00 & 100 & 120 \\
B-AL5a & B3 & 36.01 & 24.62 & 900.01 & 100 & 120 \\
B-AL6a & B3 & 35.99 & 30.77 & 900.01 & 100 & 120 \\
B-AL7a & B3 & 36.01 & 36.93 & 900.00 & 100 & 120 \\
B-AL8a & B3 & 36.00 & 49.23 & 900.01 & 100 & 120 \\
B-AL9a & B3 & 36.01 & 61.54 & 900.00 & 100 & 120 \\
B-AL10a & B3 & 36.00 & 73.85 & 900.00 & 100 & 120 \\
\hline
\end{tabular}

All of the tests in Table 4.1 and Table 4.2 were performed by placing $900 \mathrm{~g}$ of $5 \mathrm{M} \mathrm{NaOH}$ and the required amount of gibbsite in the test vessel, attaching the lid and heating to the leaching temperature over approximately 1 to 2 hours while stirring. When the vessel reached the target leaching temperature $\pm 2{ }^{\circ} \mathrm{C}$ and all of the gibbsite was completely dissolved, the initial sample was taken, and the boehmite was added. Samples of approximately $5-\mathrm{mL}$ were taken with a $10-\mathrm{mL}$ syringe through a sample port while stirring at $1,2,4,8,24,30$, and 48 hours and then filtered through a 0.45 - $\mu \mathrm{m}$ syringe filter immediately. At the end of the first 10 tests shown in Table 4.1, the remaining solids were removed, washed three times with $0.01 \mathrm{M} \mathrm{NaOH}$, dried, and weighed to determine how much solids were remaining for a material balance. The dry solids weights are shown in Table 4.3. The supernate samples were then analyzed by ICP-AES for Al and Na concentration, and the results are shown in Table 4.4 through Table 4.7 for both sets of tests. The model that was derived from this work is described and demonstrated in Appendix A.

Table 4.3. Dry-Solids Weight of Boehmite from the Model Input Leaching Tests

\begin{tabular}{cccc}
\hline Test ID & Solids wt $(\mathrm{g})$ & Test ID & Solids wt $(\mathrm{g})$ \\
\hline B-AL1 & 6.89 & B-AL6 & 16.48 \\
B-AL2 & 9.82 & B-AL7 & 14.61 \\
B-AL3 & 11.46 & B-AL8 & 25.03 \\
B-AL4 & 13.64 & B-AL9 & 27.77 \\
B-AL5 & 15.84 & B-AL10 & 30.48 \\
\hline
\end{tabular}


Table 4.4. Al Results for Filtrate from the Initial Set of Boehmite Model Input Leaching Tests

\begin{tabular}{|c|c|c|c|c|c|c|c|c|c|c|}
\hline Time (hr) & $\begin{array}{l}\text { B-AL1 (mg } \\
\text { Al/kg soln) }\end{array}$ & $\begin{array}{l}\text { B-AL2 (mg } \\
\text { Al/kg soln) }\end{array}$ & $\begin{array}{l}\text { B-AL3 (mg } \\
\text { Al/kg soln) }\end{array}$ & $\begin{array}{l}\text { B-AL4 (mg } \\
\text { Al/kg soln) }\end{array}$ & $\begin{array}{l}\text { B-AL5 (mg } \\
\text { Al/kg soln) }\end{array}$ & $\begin{array}{l}\text { B-AL6 (mg } \\
\text { Al/kg soln) }\end{array}$ & $\begin{array}{l}\text { B-AL7 (mg } \\
\text { Al/kg soln) }\end{array}$ & $\begin{array}{l}\text { B-AL8 (mg } \\
\text { Al/kg soln) }\end{array}$ & $\begin{array}{l}\text { B-AL9 (mg } \\
\text { Al/kg soln) }\end{array}$ & $\begin{array}{c}\text { B-AL10 (mg } \\
\text { Al/kg soln) }\end{array}$ \\
\hline 0 & $<4.14$ & 2,010 & 3,870 & 5,760 & 7,640 & 9,260 & 11,200 & 14,400 & 17,100 & 20,800 \\
\hline 1 & 1,390 & 2,820 & 4,450 & 6,670 & 7,860 & 9,770 & 11,400 & 14,400 & 16,300 & 21,200 \\
\hline 2 & 2,540 & 3,690 & 5,190 & 6,860 & 7,840 & 10,100 & 11,700 & 14,500 & 16,600 & 21,400 \\
\hline 4 & 4,440 & 4,930 & 6,340 & 7,800 & 8,410 & 11,200 & 10,300 & 15,300 & 18,400 & 21,900 \\
\hline 8 & 7,030 & 7,220 & 8,560 & 9,730 & 10,300 & 12,600 & 13,500 & 15,900 & 18,300 & 21,600 \\
\hline 24 & 12,400 & 12,600 & 12,700 & 14,500 & 14,800 & 17,700 & 18,000 & 19,500 & 19,800 & 19,000 \\
\hline 30 & 13,700 & 11,400 & 13,400 & 15,100 & 16,200 & 19,100 & 20,200 & 20,400 & 20,800 & 23,700 \\
\hline 48 & 17,100 & 17,300 & 16,200 & 17,400 & 19,000 & 23,000 & 23,200 & 24,400 & 23,100 & 26,200 \\
\hline Final & 17,400 & 16,800 & 16,000 & 17,700 & 19,300 & 23,400 & 23,500 & 24,400 & 22,300 & 25,400 \\
\hline
\end{tabular}

Table 4.4 (cont)

\begin{tabular}{|c|c|c|c|c|c|c|c|c|c|c|}
\hline Time (hr) & $\begin{array}{l}\text { B-AL11 (mg } \\
\text { Al/kg soln) }\end{array}$ & $\begin{array}{c}\text { B-AL12 (mg } \\
\text { Al/kg soln) }\end{array}$ & $\begin{array}{c}\text { B-AL13 (mg } \\
\text { Al/kg soln) }\end{array}$ & $\begin{array}{c}\text { B-AL14 (mg } \\
\text { Al/kg soln) }\end{array}$ & $\begin{array}{l}\text { B-AL15 (mg } \\
\text { Al/kg soln) }\end{array}$ & $\begin{array}{l}\text { B-AL16 (mg } \\
\text { Al/kg soln) }\end{array}$ & $\begin{array}{c}\text { B-AL17 (mg } \\
\text { Al/kg soln) }\end{array}$ & $\begin{array}{c}\text { B-AL18 (mg } \\
\text { Al/kg soln) }\end{array}$ & $\begin{array}{c}\text { B-AL19 (mg } \\
\text { Al/kg soln) }\end{array}$ & $\begin{array}{c}\text { B-AL20 (mg } \\
\text { Al/kg soln) }\end{array}$ \\
\hline 0 & $<4.38$ & $<3.66$ & 7.59 & $<3.92$ & $<3.78$ & $<3.85$ & 3,660 & 7,140 & 11,000 & 15,200 \\
\hline 0.25 & 253 & 146 & 115 & 895 & 647 & 288 & 3,740 & 7,680 & 11,100 & 15,000 \\
\hline 0.50 & 429 & 265 & 193 & 1,860 & 1,150 & 527 & 3,670 & 7,710 & 11,100 & 15,300 \\
\hline 0.75 & 614 & 370 & 263 & 2,710 & 1,580 & 764 & 3,950 & 7,730 & 10,900 & 15,000 \\
\hline 1 & 802 & 473 & 332 & 3,480 & 1,770 & 917 & 3,950 & 7,590 & 11,700 & 15,300 \\
\hline 2 & 1,410 & 847 & 528 & 5,870 & 3,490 & 1,740 & 3,990 & 7,790 & 12,100 & 15,000 \\
\hline 4 & 2,580 & 1,490 & 912 & 9,320 & 5,930 & 3,210 & 4,200 & 7,980 & 11,200 & 15,100 \\
\hline 8 & 4,090 & 2,480 & 1,470 & 12,700 & 9,360 & 5,600 & 4,640 & 7,750 & 11,700 & 14,900 \\
\hline 24 & 7,780 & 5,510 & 3,460 & 18,000 & 15,300 & 10,700 & 6,590 & 9,260 & 13,100 & 14,600 \\
\hline 30 & 8,920 & 6,350 & 4,150 & 18,100 & 16,000 & 11,200 & 7,170 & 9,850 & 13,000 & 15,400 \\
\hline 48 & 11,200 & 8,290 & 5,800 & 20,100 & 17,700 & 12,400 & 8,420 & 11,400 & 13,800 & 16,200 \\
\hline Final & 10,500 & 8,320 & 5,650 & 19,600 & 18,300 & 13,400 & 8,770 & 11,400 & 13,700 & 16,400 \\
\hline
\end{tabular}


Table 4.5. Na Results for Filtrate from the Initial Set of Boehmite Model Input Leaching Tests

\begin{tabular}{|c|c|c|c|c|c|c|c|c|c|c|}
\hline Time (hr) & $\begin{array}{l}\mathrm{B}-\mathrm{AL} 1 \text { (mg } \\
\mathrm{Na} / \mathrm{kg} \text { soln) }\end{array}$ & $\begin{array}{l}\mathrm{B}-\mathrm{AL} 2(\mathrm{mg} \\
\mathrm{Na} / \mathrm{kg} \text { soln })\end{array}$ & $\begin{array}{l}\mathrm{B}-\mathrm{AL} 3 \text { (mg } \\
\mathrm{Na} / \mathrm{kg} \text { soln) }\end{array}$ & $\begin{array}{l}\text { B-AL4 (mg } \\
\mathrm{Na} / \mathrm{kg} \text { soln) }\end{array}$ & $\begin{array}{l}\text { B-AL5 (mg } \\
\text { Na/kg soln) }\end{array}$ & $\begin{array}{l}\text { B-AL6 (mg } \\
\mathrm{Na} / \mathrm{kg} \text { soln) }\end{array}$ & $\begin{array}{l}\text { B-AL7 (mg } \\
\mathrm{Na} / \mathrm{kg} \text { soln) }\end{array}$ & $\begin{array}{l}\text { B-AL8 (mg } \\
\mathrm{Na} / \mathrm{kg} \text { soln) }\end{array}$ & $\begin{array}{l}\text { B-AL9 (mg } \\
\mathrm{Na} / \mathrm{kg} \text { soln) }\end{array}$ & $\begin{array}{c}\text { B-AL10 (mg } \\
\mathrm{Na} / \mathrm{kg} \text { soln) }\end{array}$ \\
\hline 0 & 87,900 & 86,500 & 89,400 & 90,100 & 88,100 & 86,900 & 87,100 & 85,800 & 80,800 & 78,400 \\
\hline 1 & 89,400 & 92,000 & 87,100 & 92,700 & 89,000 & 88,700 & 86,600 & 84,900 & 75,300 & 81,600 \\
\hline 2 & 87,800 & 92,000 & 88,700 & 87,600 & 87,200 & 88,000 & 86,900 & 85,200 & 77,600 & 81,800 \\
\hline 4 & 91,900 & 89,400 & 89,500 & 88,500 & 84,900 & 88,900 & 71,100 & 87,800 & 82,900 & 82,900 \\
\hline 8 & 94,800 & 90,200 & 93,700 & 90,800 & 88,700 & 91,300 & 86,200 & 88,700 & 80,700 & 82,300 \\
\hline 24 & 98,100 & 96,100 & 89,900 & 92,100 & 89,100 & 96,400 & 89,100 & 93,900 & 81,300 & 74,100 \\
\hline 30 & 100,000 & 79,100 & 87,900 & 89,900 & 91,500 & 97,400 & 92,800 & 95,000 & 82,700 & 85,500 \\
\hline 48 & 105,000 & 104,000 & 91,300 & 90,500 & 95,100 & 101,000 & 93,000 & 103,000 & 85,800 & 90,600 \\
\hline Final & 107,000 & 101,000 & 90,500 & 92,200 & 97,100 & 104,000 & 94,500 & 104,000 & 81,800 & 89,000 \\
\hline
\end{tabular}

Table 4.5 (cont)

\begin{tabular}{|c|c|c|c|c|c|c|c|c|c|c|}
\hline Time (hr) & $\begin{array}{l}\text { B-AL11 (mg } \\
\mathrm{Na} / \mathrm{kg} \text { soln) }\end{array}$ & $\begin{array}{l}\text { B-AL12 (mg } \\
\mathrm{Na} / \mathrm{kg} \text { soln) }\end{array}$ & $\begin{array}{l}\text { B-AL13 (mg } \\
\mathrm{Na} / \mathrm{kg} \text { soln) }\end{array}$ & $\begin{array}{l}\text { B-AL14 (mg } \\
\mathrm{Na} / \mathrm{kg} \text { soln) }\end{array}$ & $\begin{array}{l}\text { B-AL15 (mg } \\
\mathrm{Na} / \mathrm{kg} \text { soln) }\end{array}$ & $\begin{array}{l}\text { B-AL16 (mg } \\
\text { Na/kg soln) }\end{array}$ & $\begin{array}{c}\text { B-AL17 (mg } \\
\mathrm{Na} / \mathrm{kg} \text { soln) }\end{array}$ & $\begin{array}{l}\text { B-AL18 (mg } \\
\mathrm{Na} / \mathrm{kg} \text { soln) }\end{array}$ & $\begin{array}{l}\text { B-AL19 (mg } \\
\text { Na/kg soln) }\end{array}$ & $\begin{array}{c}\text { B-AL20 (mg } \\
\mathrm{Na} / \mathrm{kg} \text { soln) }\end{array}$ \\
\hline 0 & 87,600 & 90,300 & 90,800 & 92,800 & 91,400 & 90,500 & 89,000 & 81,300 & 82,800 & 88,700 \\
\hline 0.25 & 91,400 & 89,800 & 90,600 & 87,400 & 87,400 & 92,500 & 89,000 & 86,600 & 83,400 & 88,400 \\
\hline 0.50 & 88,600 & 90,800 & 89,300 & 92,100 & 90,700 & 92,400 & 86,600 & 87,000 & 83,500 & 89,200 \\
\hline 0.75 & 91,600 & 88,900 & 89,500 & 92,600 & 90,700 & 92,700 & 91,600 & 86,600 & 83,000 & 89,400 \\
\hline 1 & 92,400 & 89,400 & 91,600 & 92,000 & 81,600 & 91,400 & 92,000 & 85,000 & 89,600 & 89,900 \\
\hline 2 & 92,900 & 88,500 & 87,600 & 92,900 & 92,200 & 88,300 & 90,500 & 85,400 & 91,600 & 87,000 \\
\hline 4 & 93,000 & 89,800 & 91,000 & 91,900 & 90,800 & 88,400 & 89,800 & 86,400 & 85,300 & 89,000 \\
\hline 8 & 95,900 & 89,000 & 85,100 & 88,900 & 91,700 & 88,400 & 89,700 & 80,700 & 88,000 & 87,200 \\
\hline 24 & 94,100 & 91,300 & 91,600 & 103,000 & 95,900 & 92,500 & 92,300 & 84,900 & 92,300 & 83,200 \\
\hline 30 & 96,400 & 93,600 & 91,400 & 102,000 & 94,200 & 87,700 & 92,200 & 85,600 & 90,300 & 88,000 \\
\hline 48 & 99,300 & 93,300 & 93,700 & 109,000 & 97,800 & 86,700 & 91,000 & 90,300 & 89,700 & 90,400 \\
\hline Final & 92,900 & 95,500 & 92,800 & 106,000 & 103,000 & 91,200 & 93,500 & 90,400 & 90,300 & 91,500 \\
\hline
\end{tabular}


Table 4.6. Al Results for Filtrate from the Second Set of Boehmite Model Input Leaching Tests

\begin{tabular}{|c|c|c|c|c|c|c|c|c|c|c|}
\hline Time (hr) & $\begin{array}{c}\text { B-AL1a (mg } \\
\text { Al/kg soln) }\end{array}$ & $\begin{array}{c}\text { B-AL2a (mg } \\
\text { Al/kg soln) }\end{array}$ & $\begin{array}{c}\text { B-AL3a (mg } \\
\text { Al/kg soln) }\end{array}$ & $\begin{array}{c}\text { B-AL4a (mg } \\
\text { Al/kg soln) }\end{array}$ & $\begin{array}{c}\text { B-AL5a (mg } \\
\text { Al/kg soln) }\end{array}$ & $\begin{array}{c}\text { B-AL6a (mg } \\
\text { Al/kg soln) }\end{array}$ & $\begin{array}{c}\text { B-AL 7a (mg } \\
\text { Al/kg soln) }\end{array}$ & $\begin{array}{c}\text { B-AL8a (mg } \\
\text { Al/kg soln) }\end{array}$ & $\begin{array}{c}\text { B-AL9a (mg } \\
\text { Al/kg soln) }\end{array}$ & $\begin{array}{c}\text { B-AL10a (mg } \\
\text { Al/kg soln) }\end{array}$ \\
\hline 0 & $<3.62$ & 2,210 & 4,410 & 6,840 & 9,060 & 11,200 & 12,900 & 17,600 & 22,000 & 26,200 \\
\hline 0.25 & 543 & 2,490 & 4,630 & 6,610 & 8,800 & 11,100 & 13,500 & 17,600 & 20,800 & 26,200 \\
\hline 0.50 & 950 & 2,600 & 5,050 & 6,950 & 9,160 & 11,200 & 13,400 & 17,400 & 21,000 & 26,400 \\
\hline 0.75 & 1,320 & 2,770 & 4,770 & 7,060 & 9,160 & 11,400 & 13,800 & 17,700 & 20,800 & 25,700 \\
\hline 1 & 1,610 & 2,940 & 5,300 & 7,280 & 8,860 & 11,000 & 13,000 & 18,100 & 21,000 & 27,200 \\
\hline 2 & 2,850 & 3,870 & 5,990 & 7,570 & 9,440 & 11,700 & 13,800 & 16,700 & 21,000 & 27,400 \\
\hline 4 & 4,720 & 5,380 & 7,280 & 8,060 & 10,200 & 12,700 & 14,400 & 18,100 & 21,600 & 27,300 \\
\hline 8 & 7,400 & 7,910 & 9,220 & 10,000 & 11,600 & 13,600 & 15,600 & 18,600 & 21,900 & 26,500 \\
\hline 24 & 12,800 & 13,000 & 13,500 & 14,400 & 15,400 & 17,800 & 18,900 & 20,900 & 23,700 & 28,200 \\
\hline 30 & 14,900 & 14,800 & 14,600 & 16,200 & 16,600 & 19,100 & 18,800 & 21,100 & 23,900 & 29,100 \\
\hline 48 & 16,900 & 16,800 & 17,900 & 18,200 & 18,700 & 24,400 & 20,600 & 22,100 & 26,400 & 31,200 \\
\hline Final & 17,300 & 15,900 & 17,900 & 17,700 & 19,100 & 23,900 & 20,700 & 22,800 & 28,000 & 31,400 \\
\hline
\end{tabular}

Table 4.7. Na Results for Filtrate from the Second Set of Boehmite Model Input Leaching Tests

\begin{tabular}{|c|c|c|c|c|c|c|c|c|c|c|}
\hline Time (hr) & $\begin{array}{c}\text { B-ALla (mg } \\
\mathrm{Na} / \mathrm{kg} \text { soln) }\end{array}$ & $\begin{array}{c}\text { B-AL2a (mg } \\
\mathrm{Na} / \mathrm{kg} \text { soln) }\end{array}$ & $\begin{array}{c}\text { B-AL3a (mg } \\
\mathrm{Na} / \mathrm{kg} \text { soln) }\end{array}$ & $\begin{array}{c}\text { B-AL4a (mg } \\
\mathrm{Na} / \mathrm{kg} \text { soln) }\end{array}$ & $\begin{array}{l}\text { B-AL5a (mg } \\
\mathrm{Na} / \mathrm{kg} \text { soln) }\end{array}$ & $\begin{array}{l}\text { B-AL6a (mg } \\
\mathrm{Na} / \mathrm{kg} \text { soln) }\end{array}$ & $\begin{array}{l}\mathrm{B}-\mathrm{AL} 7 \mathrm{a}(\mathrm{mg} \\
\mathrm{Na} / \mathrm{kg} \text { soln) }\end{array}$ & $\begin{array}{c}\text { B-AL8a } \\
\text { (mg Na/kg } \\
\text { soln) }\end{array}$ & $\begin{array}{c}\text { B-AL9a (mg } \\
\mathrm{Na} / \mathrm{kg} \text { soln) }\end{array}$ & $\begin{array}{c}\text { B-AL10a (mg } \\
\text { Na/kg soln) }\end{array}$ \\
\hline 0 & 95,800 & 87,700 & 88,000 & 88,200 & 87,900 & 87,600 & 83,700 & 88,400 & 83,600 & 85,600 \\
\hline 0.25 & 90,100 & 93,100 & 88,900 & 85,400 & 84,800 & 85,900 & 84,100 & 85,000 & 82,600 & 85,000 \\
\hline 0.50 & 91,800 & 90,900 & 90,200 & 88,900 & 87,300 & 86,500 & 83,000 & 86,100 & 83,400 & 85,400 \\
\hline 0.75 & 93,700 & 90,800 & 87,800 & 87,600 & 86,700 & 87,400 & 87,500 & 84,800 & 80,400 & 83,000 \\
\hline 1 & 91,700 & 88,600 & 89,100 & 89,500 & 84,100 & 84,600 & 81,200 & 85,300 & 81,800 & 88,500 \\
\hline 2 & 94,300 & 90,800 & 90,000 & 88,400 & 86,500 & 88,300 & 85,300 & 79,000 & 82,400 & 89,100 \\
\hline 4 & 91,600 & 90,000 & 90,900 & 83,800 & 87,000 & 86,400 & 85,900 & 85,700 & 83,600 & 89,000 \\
\hline 8 & 92,300 & 92,500 & 91,500 & 87,500 & 88,200 & 87,100 & 87,600 & 86,700 & 83,800 & 87,000 \\
\hline 24 & 98,500 & 91,000 & 89,200 & 87,200 & 87,300 & 94,800 & 87,100 & 88,900 & 88,200 & 92,100 \\
\hline 30 & 101,000 & 92,800 & 88,600 & 90,200 & 88,500 & 96,700 & 86,500 & 87,300 & 85,900 & 93,800 \\
\hline 48 & 105,000 & 95,100 & 95,300 & 89,300 & 89,400 & 103,000 & 86,400 & 86,200 & 92,400 & 101,000 \\
\hline Final & 107,000 & 90,100 & 95,600 & 87,000 & 91,300 & 101,000 & 86,700 & 89,400 & 92,200 & 102,000 \\
\hline
\end{tabular}





\subsection{PEP Support Leaching Tests}

The objective of these tests was to determine the effect of temperature and aeration on the extent of chromium dissolution during caustic leaching and to determine the rate and extent of dissolution of aluminum under UFP-T01A/B caustic leaching conditions.

Tests specified in ICN-TP-RPP-WTP-509_R0.3 were performed according to the test matrix shown in Table 5.1. WTP staff were responsible for interpreting these data and for assessing the impact of the data on planned PEP operations. As such, the data are presented herein, but there is no interpretation provided.

In addition, a second set of tests was required so the current kinetics model for boehmite dissolution in the presence of gibbsite (predictions) could be confirmed before the PEP testing. These tests were specified in ICN-TP-RPP-WTP-509_R0.5. These tests were performed according to the test matrix shown in Table 5.2. Hydroxide concentration was not measured as it was assumed that the amount added was correct. WTP staff were responsible for interpreting these data and for assessing the impact of the data on planned PEP operations. As such, the data are presented herein, but there is no interpretation provided. Also note that there was some evaporation of water throughout the test; however, no attempt has been made to determine the quantity of water evaporated or to perform a material balance for these tests.

Table 5.1. Test Matrix for the PEP Support Leaching Tests

\begin{tabular}{ccccccc}
\hline & $\begin{array}{c}\text { Initial Wt\% } \\
\text { Solids }\end{array}$ & $\begin{array}{c}\text { 250 Gal Batch } \\
\text { PEP Simulant } \\
(\mathrm{g})\end{array}$ & $\begin{array}{c}\text { CrOOH } \\
\text { Slurry } \\
(\mathrm{g})\end{array}$ & $\begin{array}{c}19 \mathrm{M} \mathrm{NaOH} \\
(\mathrm{g})\end{array}$ & $\begin{array}{c}\text { Leach } \\
\text { DI water }(\mathrm{g})\end{array}$ & $\begin{array}{c}\text { Temperature }\left({ }^{\circ} \mathrm{C}\right) \\
\text { Test }\end{array}$ \\
\hline PST-1 & 20 & 481.90 & 118.16 & 381.83 & 105.41 & 100 \\
PST-2 & 20 & 481.90 & 118.16 & 381.83 & 105.41 & 95 \\
PST-3 & 20 & 481.90 & 118.16 & 381.83 & 105.41 & 85 \\
PST-4 & 20 & 481.90 & 118.16 & 381.83 & 105.41 & 80 \\
PST-5 & 5 & 596.34 & 40.62 & 192.27 & 100.63 & 100 \\
PST-6 & 5 & 596.34 & 40.62 & 192.27 & 100.63 & 95 \\
PST-7 & 5 & 596.34 & 40.62 & 192.27 & 100.63 & 85 \\
PST-8 & 5 & 596.34 & 40.62 & 192.27 & 100.63 & 80 \\
PST-9 & 20 & 481.90 & 118.16 & 381.83 & 105.41 & 85 \\
\hline
\end{tabular}

Table 5.2. Test Matrix for the PEP Support Leaching Tests \#2

\begin{tabular}{cccccc}
\hline Test ID & $\begin{array}{c}\text { Initial Wt } \% \\
\text { Solids }\end{array}$ & $\begin{array}{c}250 \text { Gal Batch PEP } \\
\text { Simulant }(\mathrm{g})\end{array}$ & $\begin{array}{c}19 \mathrm{M} \mathrm{NaOH} \\
(\mathrm{g})\end{array}$ & $\begin{array}{c}\text { Leach Temperature } \\
\left({ }^{\circ} \mathrm{C}\right)\end{array}$ \\
\hline PST2-1 & 5 & 576.01 & 145.04 & 79.02 & 98 \\
PST2-2 & 20 & 449.01 & 261.02 & 90.08 & 98 \\
PST2-3 & 5 & 513.00 & 209.01 & 78.01 & 80 \\
PST2-4 & 20 & 332.00 & 379.00 & 89.08 & 80 \\
PST2-5 & 5 & 538.01 & 182.98 & 79.01 & 98 \\
PST2-6 & 5 & 510.00 & 211.99 & 78.02 & 85 \\
PST2-7 & 20 & 388.01 & 323.02 & 89.02 & 85 \\
\hline
\end{tabular}


All of these tests were performed by placing the correct amount of PEP simulant with the appropriate wt\% UDS into the test vessel. The CrOOH slurry (if needed), deionized (DI) water, and $19 \mathrm{M} \mathrm{NaOH}$ were added to it. The lid was attached, and stirring at $120 \mathrm{rpm}$ was initiated. PST-9 was aerated at $\sim 85-\mathrm{mL} / \mathrm{min}$ per the rotometer reading at laboratory conditions during the test with air being injected into the slurry below the surface near the bottom of the vessel. Two initial samples were removed (one as supernatant and one as slurry), and then it was heated to temperature over a 6-hour timeframe. When the test vessel reached the appropriate temperature after 6 hours, another sample was removed as the time 0 sample. Then 5-mL supernatant samples were obtained while stirring at 1,2, 4, 8, 12, 16, 20, and 24 hours by filtering slurry through a $0.45-\mu \mathrm{m}$ syringe filter immediately for both sets of tests as well as 3,5 , and 6 hours for the second set of tests. The slurry samples were washed three times with $0.01 \mathrm{M}$ $\mathrm{NaOH}$ and were then analyzed by ICP-AES for $\mathrm{Al}, \mathrm{Cr}$, and $\mathrm{Na}$ concentration. The results from the slurry samples from the first set of tests are shown in Table 5.3. The supernate was also analyzed for $\mathrm{Al}, \mathrm{Cr}$, and $\mathrm{Na}$ by ICP-AES, and the results are shown in Table 5.4 through Table 5.6 for the first set of tests. The slurry samples from the second set of tests were analyzed by ICP-AES for Al, Fe, and $\mathrm{Na}$ and the results are shown in Table 5.7. The supernatant samples from the second set of tests were analyzed by ICP-AES for $\mathrm{Al}$ and $\mathrm{Na}$ with the results shown in Table 5.8 and Table 5.9. 
Table 5.3. Results for Solids from the PEP Support Leaching Tests

\begin{tabular}{|c|c|c|c|c|c|c|c|c|c|}
\hline & $\begin{array}{c}\text { PST-1-INS } \\
\text { (mg/kg } \\
\text { slurry) } \\
\end{array}$ & $\begin{array}{c}\text { PST-2-INS } \\
\text { (mg/kg } \\
\text { slurry) }\end{array}$ & $\begin{array}{c}\text { PST-3-INS } \\
\text { (mg/kg slurry) }\end{array}$ & $\begin{array}{c}\text { PST-4-INS } \\
\text { (mg/kg slurry) }\end{array}$ & $\begin{array}{c}\text { PST-5-INS } \\
\text { (mg/kg slurry) }\end{array}$ & $\begin{array}{c}\text { PST-6-FIS } \\
\text { (mg/kg } \\
\text { slurry) } \\
\end{array}$ & $\begin{array}{c}\text { PST-7-FIS } \\
\text { (mg/kg } \\
\text { slurry) } \\
\end{array}$ & $\begin{array}{c}\text { PST-8-FIS } \\
\text { (mg/kg } \\
\text { slurry) }\end{array}$ & $\begin{array}{c}\text { PST-9-FIS } \\
\text { (mg/kg } \\
\text { slurry) }\end{array}$ \\
\hline $\mathrm{Al}$ & 19,600 & 19,300 & 17,900 & 18,300 & 9,040 & 8,880 & 9,020 & 9,030 & 20,600 \\
\hline $\mathrm{Cr}$ & 1,020 & 1,020 & 1,030 & 1,010 & 407 & 400 & 392 & 406 & 1,005 \\
\hline $\mathrm{Na}$ & 139,000 & 135,000 & 139,000 & 134,000 & 115,000 & 113,000 & 112,000 & 111,000 & 132,000 \\
\hline
\end{tabular}

Table 5.3 (cont)

\begin{tabular}{|c|c|c|c|c|c|c|c|c|c|}
\hline & $\begin{array}{l}\text { PST-1-FWS } \\
\text { (mg/kg slurry) }\end{array}$ & $\begin{array}{l}\text { PST-2-FWS } \\
\text { (mg/kg slurry) }\end{array}$ & $\begin{array}{l}\text { PST-3-FWS } \\
\text { (mg/kg slurry) }\end{array}$ & $\begin{array}{l}\text { PST-4-FWS } \\
\text { (mg/kg slurry) }\end{array}$ & $\begin{array}{l}\text { PST-5-FWS } \\
\text { (mg/kg slurry) }\end{array}$ & $\begin{array}{l}\text { PST-6-FWS } \\
\text { (mg/kg slurry) }\end{array}$ & $\begin{array}{l}\text { PST-7-FWS } \\
\text { (mg/kg slurry) }\end{array}$ & $\begin{array}{l}\text { PST-8-FWS } \\
\text { (mg/kg slurry) }\end{array}$ & $\begin{array}{c}\text { PST-9-FWS } \\
\text { (mg/kg } \\
\text { slurry) }\end{array}$ \\
\hline $\mathrm{Al}$ & 83,600 & 89,400 & 110,000 & 104,000 & 63,800 & 68,200 & 69,900 & 63,700 & 94,600 \\
\hline $\mathrm{Cr}$ & 4,690 & 4,440 & 4,340 & 4,210 & 2,610 & 2,010 & 2,720 & 2,700 & 3,620 \\
\hline $\mathrm{Na}$ & 19,500 & 12,800 & 11,200 & 18,400 & 3,220 & 17,800 & 3,710 & 2,390 & 5,670 \\
\hline
\end{tabular}

Table 5.4. Al Results for Filtrate from the PEP Support Leaching Tests

\begin{tabular}{|c|c|c|c|c|c|c|c|c|c|}
\hline Time (hr) & $\begin{array}{l}\text { PST-1 (mg } \\
\mathrm{Al} / \mathrm{kg} \text { soln) }\end{array}$ & $\begin{array}{l}\text { PST-2 (mg } \\
\mathrm{Al} / \mathrm{kg} \text { soln) }\end{array}$ & $\begin{array}{l}\text { PST-3 (mg } \\
\text { Al/kg soln) }\end{array}$ & $\begin{array}{l}\text { PST-4 (mg } \\
\text { Al/kg soln) }\end{array}$ & $\begin{array}{l}\text { PST-5 (mg } \\
\text { Al/kg soln) }\end{array}$ & $\begin{array}{l}\text { PST-6 (mg } \\
\text { Al/kg soln) }\end{array}$ & $\begin{array}{l}\text { PST-7 (mg } \\
\text { Al/kg soln) }\end{array}$ & $\begin{array}{l}\text { PST-8 (mg } \\
\text { Al/kg soln) }\end{array}$ & $\begin{array}{l}\text { PST-9 (mg } \\
\mathrm{Al} / \mathrm{kg} \text { soln) }\end{array}$ \\
\hline Initial & 5,450 & 4,890 & 5,020 & 4,980 & 2,570 & 2,730 & 2,630 & 2,680 & 5,560 \\
\hline 0 & 12,300 & 12,100 & 10,600 & 10,700 & 5,440 & 5,430 & 5,430 & 5,540 & 10,700 \\
\hline 1 & 12,600 & 12,300 & 11,000 & 10,600 & 5,550 & 5,820 & 4,990 & 5,260 & 10,600 \\
\hline 2 & 12,500 & 12,700 & 11,100 & 10,900 & 5,620 & 5,470 & 5,340 & 5,200 & 10,600 \\
\hline 4 & 13,300 & 11,800 & 11,100 & 11,100 & 5,930 & 5,830 & 5,300 & 5,680 & 10,900 \\
\hline 8 & 13,400 & 12,600 & 11,100 & 11,200 & 6,080 & 5,830 & 5,420 & 5,430 & 11,400 \\
\hline 12 & 16,300 & 14,100 & 11,300 & 11,100 & 6,610 & 6,100 & 5,580 & 5,480 & 11,800 \\
\hline 16 & 17,500 & 15,600 & 11,600 & 11,200 & 6,790 & 6,530 & 5,940 & 5,610 & 12,200 \\
\hline 20 & 18,700 & 16,600 & 11,700 & 11,200 & 7,420 & 6,920 & 5,860 & 5,670 & 12,300 \\
\hline 24 & 20,000 & 17,900 & 12,200 & 11,500 & 7,700 & 7,470 & 5,740 & 5,770 & 13,100 \\
\hline Final & 18,400 & 15,600 & 11,400 & 11,300 & 7,570 & 7,150 & 5,520 & 5,670 & 13,400 \\
\hline
\end{tabular}


Table 5.5. Cr Results for Filtrate from the PEP Support Leaching Tests

\begin{tabular}{|c|c|c|c|c|c|c|c|c|c|}
\hline Time (hr) & $\begin{array}{l}\text { PST-1 (mg } \\
\text { Cr/kg soln) }\end{array}$ & $\begin{array}{l}\text { PST-2 (mg } \\
\mathrm{Cr} / \mathrm{kg} \text { soln) }\end{array}$ & $\begin{array}{l}\text { PST-3 (mg } \\
\text { Cr/kg soln) }\end{array}$ & $\begin{array}{l}\text { PST-4 (mg } \\
\text { Cr/kg soln) }\end{array}$ & $\begin{array}{l}\text { PST-5 (mg } \\
\text { Cr/kg soln) }\end{array}$ & $\begin{array}{l}\text { PST-6 (mg } \\
\text { Cr/kg soln) }\end{array}$ & $\begin{array}{l}\text { PST-7 (mg } \\
\text { Cr/kg soln) }\end{array}$ & $\begin{array}{l}\text { PST-8 (mg } \\
\text { Cr/kg soln) }\end{array}$ & $\begin{array}{l}\text { PST-9 (mg } \\
\text { Cr/kg soln) }\end{array}$ \\
\hline Initial & 57.6 & 58.5 & 62.8 & 60.8 & 12.1 & 17.6 & 17.3 & 17.8 & 79.3 \\
\hline 0 & 403 & 387 & 344 & 323 & 161 & 155 & 135 & 123 & 376 \\
\hline 1 & 418 & 398 & 384 & 356 & 177 & 181 & 147 & 140 & 407 \\
\hline 2 & 422 & 424 & 397 & 378 & 187 & 184 & 167 & 154 & 422 \\
\hline 4 & 452 & 449 & 417 & 397 & 211 & 213 & 181 & 187 & 454 \\
\hline 8 & 457 & 494 & 454 & 433 & 236 & 237 & 218 & 207 & 523 \\
\hline 12 & 553 & 520 & 490 & 454 & 274 & 271 & 246 & 235 & 572 \\
\hline 16 & 591 & 575 & 525 & 487 & 278 & 304 & 282 & 256 & 625 \\
\hline 20 & 629 & 614 & 562 & 529 & 309 & 332 & 291 & 275 & 655 \\
\hline 24 & 660 & 669 & 608 & 562 & 321 & 351 & 297 & 294 & 724 \\
\hline Final & 657 & 635 & 593 & 549 & 319 & 343 & 286 & 284 & 723 \\
\hline
\end{tabular}

Table 5.6. Na Results for Filtrate from the PEP Support Leaching Tests

\begin{tabular}{|c|c|c|c|c|c|c|c|c|c|}
\hline Time (hr) & $\begin{array}{l}\mathrm{PST}-1 \text { (mg } \\
\mathrm{Na} / \mathrm{kg} \text { soln) }\end{array}$ & $\begin{array}{l}\text { PST-2 (mg } \\
\mathrm{Na} / \mathrm{kg} \text { soln) }\end{array}$ & $\begin{array}{l}\mathrm{PST}-3(\mathrm{mg} \\
\mathrm{Na} / \mathrm{kg} \text { soln) }\end{array}$ & $\begin{array}{l}\text { PST-4 (mg } \\
\mathrm{Na} / \mathrm{kg} \text { soln) }\end{array}$ & $\begin{array}{l}\text { PST-5 (mg } \\
\mathrm{Na} / \mathrm{kg} \text { soln) }\end{array}$ & $\begin{array}{l}\text { PST-6 (mg } \\
\mathrm{Na} / \mathrm{kg} \text { soln) }\end{array}$ & $\begin{array}{l}\text { PST-7 (mg } \\
\mathrm{Na} / \mathrm{kg} \text { soln) }\end{array}$ & $\begin{array}{l}\text { PST-8 (mg } \\
\mathrm{Na} / \mathrm{kg} \text { soln) }\end{array}$ & $\begin{array}{l}\text { PST-9 (mg } \\
\mathrm{Na} / \mathrm{kg} \text { soln) }\end{array}$ \\
\hline Initial & 140,000 & 139,000 & 142,000 & 139,000 & 114,000 & 114,000 & 112,000 & 109,000 & 136,000 \\
\hline 0 & 144,000 & 144,000 & 138,000 & 138,000 & 117,000 & 116,000 & 119,000 & 122,000 & 141,000 \\
\hline 1 & 144,000 & 142,000 & 142,000 & 138,000 & 115,000 & 124,000 & 117,000 & 118,000 & 140,000 \\
\hline 2 & 142,000 & 146,000 & 143,000 & 141,000 & 119,000 & 116,000 & 116,000 & 113,000 & 140,000 \\
\hline 4 & 144,000 & 146,000 & 143,000 & 142,000 & 116,000 & 133,000 & 113,000 & 121,000 & 141,000 \\
\hline 8 & 134,000 & 148,000 & 144,000 & 144,000 & 116,000 & 116,000 & 117,000 & 115,000 & 146,000 \\
\hline 12 & 149,000 & 144,000 & 140,000 & 140,000 & 119,000 & 119,000 & 119,000 & 117,000 & 148,000 \\
\hline 16 & 148,000 & 150,000 & 142,000 & 141,000 & 116,000 & 121,000 & 126,000 & 119,000 & 150,000 \\
\hline 20 & 152,000 & 152,000 & 141,000 & 142,000 & 116,000 & 124,000 & 119,000 & 123,000 & 149,000 \\
\hline 24 & 149,000 & 158,000 & 144,000 & 143,000 & 118,000 & 128,000 & 117,000 & 122,000 & 153,000 \\
\hline Final & 151,000 & 149,000 & 140,000 & 142,000 & 115,000 & 126,000 & 117,000 & 119,000 & 156,000 \\
\hline
\end{tabular}


Table 5.7. Results for Solids from the PEP Support Leaching Tests \#2

\begin{tabular}{|c|c|c|c|c|c|c|c|c|c|}
\hline & $\begin{array}{l}\text { PST2-1- } \\
\text { FWS (mg/kg } \\
\text { slurry) }\end{array}$ & $\begin{array}{c}\text { PST2-2- } \\
\text { FWS (mg/kg } \\
\text { slurry) }\end{array}$ & $\begin{array}{c}\text { PST2-3- } \\
\text { FWS (mg/kg } \\
\text { slurry) }\end{array}$ & $\begin{array}{c}\text { PST2-4- } \\
\text { FWS (mg/kg } \\
\text { slurry) }\end{array}$ & $\begin{array}{c}\text { PST2-5- } \\
\text { FWS (mg/kg } \\
\text { slurry) }\end{array}$ & $\begin{array}{c}\text { PST2-6- } \\
\text { FWS (mg/kg } \\
\text { slurry) }\end{array}$ & $\begin{array}{l}\text { PST2-7-FWS } \\
\text { (mg/kg slurry) }\end{array}$ & $\begin{array}{l}\text { Initial } 5-\mathrm{wt}^{\%} \% \\
\text { UDS (mg/kg } \\
\text { slurry) }\end{array}$ & $\begin{array}{c}\text { Initial } 20-\mathrm{wt}^{0} \% \\
\text { UDS (mg/kg } \\
\text { slurry) }\end{array}$ \\
\hline $\mathrm{Al}$ & 183,000 & 133,000 & 215,000 & 223,000 & 142,000 & 229,000 & 211,000 & 255,000 & 283,000 \\
\hline $\mathrm{Fe}$ & 212,000 & 228,000 & 170,000 & 170,000 & 237,000 & 155,000 & 141,000 & 114,000 & 87,300 \\
\hline $\mathrm{Na}$ & 14,000 & 29,000 & $<9,100$ & $<9,200$ & 11,000 & {$[19,000]$} & {$[24,000]$} & $<9,000$ & $<8.700$ \\
\hline
\end{tabular}

Table 5.8. Al Results for Filtrate from the PEP Support Leaching Tests \#2

\begin{tabular}{|c|c|c|c|c|c|c|c|}
\hline Time (hr) & $\begin{array}{l}\text { PST2-1 (mg } \\
\text { Al/L soln) }\end{array}$ & $\begin{array}{l}\text { PST2-2 (mg } \\
\text { Al/L soln) }\end{array}$ & $\begin{array}{l}\text { PST2-3 (mg } \\
\text { Al/L soln) }\end{array}$ & $\begin{array}{l}\text { PST2-4 (mg } \\
\text { Al/L soln) }\end{array}$ & $\begin{array}{c}\text { PST2-5 (mg A1/L } \\
\text { soln) }\end{array}$ & $\begin{array}{l}\text { PST2-6 (mg } \\
\text { A1/L soln) }\end{array}$ & $\begin{array}{c}\text { PST2-7 (mg } \\
\text { Al/L soln) }\end{array}$ \\
\hline Initial & 2,620 & 2,340 & 2,540 & 1,920 & 2,560 & 2,980 & 2,850 \\
\hline 0 & 4,020 & 4,940 & 3,780 & 3,220 & 3,930 & 5,900 & 12,300 \\
\hline 1 & 4,020 & 5,090 & 3,810 & 2,850 & 3,980 & 5,980 & 12,500 \\
\hline 2 & 4,070 & 5,370 & 3,810 & 3,250 & 4,140 & 5,960 & 12,600 \\
\hline 3 & 4,060 & 5,470 & 3,840 & 2,900 & 4,200 & 6,100 & 12,700 \\
\hline 4 & 4,260 & 5,730 & 3,860 & 3,150 & 4,280 & 6,180 & 13,000 \\
\hline 5 & 4,230 & 5,820 & 3,860 & 3,400 & 4,390 & 6,080 & 12,600 \\
\hline 6 & 4,240 & 6,000 & 3,720 & 3,540 & 4,610 & 6,230 & 13,200 \\
\hline 8 & 4,380 & 6,270 & 3,780 & 3,140 & 4,860 & 6,260 & 13,400 \\
\hline 12 & 4,640 & 6,830 & 3,830 & 3,760 & 5,180 & 6,380 & 14,000 \\
\hline 16 & 4,890 & 7,470 & 3,840 & 3,970 & 5,590 & 6,580 & 14,700 \\
\hline 20 & 5,060 & 8,120 & 3,860 & 4,150 & 5,940 & 6,660 & 15,300 \\
\hline 24 & 5,300 & 8,370 & 3,980 & 4,290 & 6,150 & 6,940 & 15,900 \\
\hline Final & 5,380 & 8,470 & 3,980 & 4,390 & 6,190 & 6,770 & 16,000 \\
\hline
\end{tabular}


Table 5.9. Na Results for Filtrate from the PEP Support Leaching Tests \#2

\begin{tabular}{|c|c|c|c|c|c|c|c|}
\hline Time (hr) & $\begin{array}{l}\text { PST2-1 (mg } \\
\mathrm{Na} / \mathrm{L} \text { soln) }\end{array}$ & $\begin{array}{c}\text { PST2-2 (mg } \\
\mathrm{Na} / \mathrm{L} \text { soln) }\end{array}$ & $\begin{array}{l}\text { PST2-3 (mg } \\
\mathrm{Na} / \mathrm{L} \text { soln) }\end{array}$ & $\begin{array}{l}\text { PST2-4 (mg } \\
\text { Na/L soln) }\end{array}$ & $\begin{array}{l}\text { PST2-5 (mg } \\
\text { Na/L soln) }\end{array}$ & $\begin{array}{c}\text { PST2-6 (mg } \\
\mathrm{Na} / \mathrm{L} \text { soln) }\end{array}$ & $\begin{array}{l}\text { PST2-7 (mg } \\
\mathrm{Na} / \mathrm{L} \text { soln) }\end{array}$ \\
\hline Initial & 140,000 & 181,000 & 164,000 & 217,000 & 152,000 & 166,000 & 204,000 \\
\hline 0 & 140,000 & 180,000 & 165,000 & 220,000 & 155,000 & 170,000 & 210,000 \\
\hline 1 & 141,000 & 178,000 & 166,000 & 218,000 & 156,000 & 170,000 & 209,000 \\
\hline 2 & 139,000 & 183,000 & 164,000 & 222,000 & 157,000 & 169,000 & 212,000 \\
\hline 3 & 140,000 & 181,000 & 167,000 & 219,000 & 160,000 & 169,000 & 212,000 \\
\hline 4 & 143,000 & 184,000 & 167,000 & 220,000 & 161,000 & 170,000 & 214,000 \\
\hline 5 & 141,000 & 183,000 & 166,000 & 222,000 & 161,000 & 171,000 & 211,000 \\
\hline 6 & 138,000 & 181,000 & 165,000 & 220,000 & 166,000 & 172,000 & 212,000 \\
\hline 8 & 143,000 & 183,000 & 165,000 & 219,000 & 168,000 & 172,000 & 215,000 \\
\hline 12 & 142,000 & 185,000 & 167,000 & 222,000 & 170,000 & 173,000 & 215,000 \\
\hline 16 & 144,000 & 186,000 & 168,000 & 226,000 & 175,000 & 172,000 & 216,000 \\
\hline 20 & 142,000 & 186,000 & 163,000 & 222,000 & 177,000 & 173,000 & 218,000 \\
\hline 24 & 145,000 & 185,000 & 169,000 & 222,000 & 176,000 & 172,000 & 218,000 \\
\hline Final & 147,000 & 191,000 & 168,000 & 220,000 & 182,000 & 171,000 & 220,000 \\
\hline
\end{tabular}




\subsection{PEP Parallel Leaching Tests}

The objectives of these tests were to provide data to support the development of scale factors between laboratory process measurements and those of PEP. These tests are specified in Test Plan TP-WTP-PEP-044, Rev. 0.2 and were carried out as specified by WTP for each PEP test that was performed.

Four laboratory-scale caustic-leach tests were performed to support the PEP functional tests (Section 6.1), and two laboratory-scale caustic-leach tests were performed to support each of PEP Test A (Section 6.2), Test B (Section 6.3), and Test D (Section 6.4). Four laboratory-scale oxidative-leach tests were performed to support PEP Test A, Test B, and Test D with one test each for PEP Test A and PEP Test B. There were two tests for PEP Test $\mathrm{D}$ with different amounts of permanganate added (Section 6.5). The analysis of test results presented in this section is reported in WTP-RPT-186 (Mahoney et al. 2009) and WTP-RPT-188 (Rapko et al. 2009). Integrated PEP Test $C$ was deleted from the scope of the testing (ICN-TP-RPP-WTP-506_R0.2).

\subsection{PEP Functional Test Caustic Leach}

The PEP Functional Test was conducted under three Test Instructions, TI-WTP-PEP-062 (TI-062), TI-WTP-PEP-067 (TI-067), and TI-WTP-PEP-032 (TI-032). The scope of TI-067 included collecting data from UFP-VSL-T01A. Data from the UFP-VSL-T01A and UFP-VSL-T02A leach processes was collected during test TI-032. TI-062 included caustic addition only.

According to TI-032, simulant stored in HLP-VSL-T22 was transferred into vessel UFP-VSL-T01A. A sample of the feed slurry (without added caustic) was taken from the inner Coriolis densitometer (CD) sample loop at the middle-elevation port in UFP-VSL-T01A for use as feed in the laboratory-scale caustic leach tests (PL_LAB_CL_3). This slurry sample was taken on December 20, 2008 at 9:23.

An anti-foaming agent (AFA), Dow Corning Q2-3183A, was added directly to UFP-VSL-T01A in a quantity expected to produce a nominal concentration of $350 \mathrm{ppm}$ in the slurry. After this addition, caustic reagent (nominally $19 \mathrm{M} \mathrm{NaOH}$ ) was injected into the feed transfer line and added directly into UFP-VSL-T01A. This was thoroughly mixed for 30 minutes, and then another sample of slurry (comprising feed plus caustic and AFA) was taken from the inner CD sample loop at the middle-elevation port in UFP-VSL-T01A for use as feed in the laboratory-scale caustic leach tests (PL_LAB_CL_1). This slurry sample was taken on December 20, 2008 at 10:38. The sample, when taken, was at $71^{\circ} \mathrm{C}$ and was rapidly cooled to ambient temperature (approximately $20^{\circ} \mathrm{C}$ to $25^{\circ} \mathrm{C}$ ) to minimize any further leaching reaction.

For the samples taken from the UFP-VSL-T02A tank, feed stored in HLP-VSL-T22 was transferred into vessel UFP-VSL-T01A. AFA was added directly to UFP-VSL-T01A in a quantity expected to produce a nominal concentration of $350 \mathrm{ppm}$ in the slurry. The slurry was then transferred from the UFP-VSL-T01A feed tank to UFP-VSL-T02A, and permeate was removed from UFP-VSL-T02A through the first ultrafilter bundle to increase the solids concentration of the slurry. As permeate was removed, the volume (and level) in UFP-VSL-T02A fell, triggering the transfer of small refill batches (i.e., $11 \mathrm{gal}$ ) of fresh simulant from UFP-VSL-T01A. The filtering and refill process continued, leaving a target quantity of slurry at about 20 -wt $\%$ UDS in the UFP-VSL-T02A vessel and filter loop. When the 
solids concentration process was complete, a sample of the feed slurry (without added caustic) was taken from the inner CD sample loop at the middle-elevation port in UFP-VSL-T02A for use as feed in the laboratory-scale caustic leach tests (PL_LAB_CL_4). This slurry sample was taken on January 2, 2009 at $7: 19$.

A solution of $19 \mathrm{M}$ caustic was added upstream of the filter loop pumps while the slurry was circulated through the loop. The caustic slurry in UFP-VSL-T02A was heated to about $71^{\circ} \mathrm{C}$ using the heat of dilution of the concentrated $\mathrm{NaOH}$ and mechanical heat from the filter loop recirculation pumps. The filter loop pumps were then turned off, and a portion of the concentrated slurry in the filter loop was flushed back into UFP-VSL-T02A before the loop was closed off from the vessel. Because the total loop volume ( 82 gal, excluding dead volumes) was significantly greater than the flush volume ( $\sim 6$ gal), none of the flush solution $(0.01 \mathrm{M} \mathrm{NaOH})$ is expected to have entered the vessel. After the flush, a sample of slurry was taken from the middle-low region of vessel UFP-VSL-T02A using the CD sampler for use as feed in the laboratory-scale caustic leach test (PL_LAB_CL_2). The sample was rapidly cooled to ambient temperature (approximately $20^{\circ} \mathrm{C}$ to $25^{\circ} \mathrm{C}$ ) to minimize any further leaching reaction. This slurry sample was taken on January 2, 2009 at 11:41.

The laboratory-scale feed was stored at laboratory ambient temperature until it was used. The delay between the time when the feed was acquired from PEP and the time laboratory-scale testing started was about 23 days for tests PL_LAB_CL_1 and PL_LAB_CL_3 (from December 20, 2008 to January 12, 2009) and about 10 days for tests PL_LAB_CL_2 and PL_LAB_CL_4 (from January 2, 2009 to January 12, 2009).

The PEP sample ID, laboratory sample ID, and the amounts of components used in the functional laboratory-scale tests are shown in Table 6.1.

Table 6.1. PEP Parallel Functional Test Matrix

\begin{tabular}{cccccc}
\hline & & PEP & 19 M & DI \\
PEP Test ID & Lab Test ID & Test Matrix & $\begin{array}{c}\text { Simutat } \\
\text { (g) }\end{array}$ & $\begin{array}{c}\text { NaOH } \\
\text { (g) }\end{array}$ & $\begin{array}{c}\text { Water } \\
(\mathrm{g})\end{array}$ \\
\hline S_01AML_020_XX_0749_CUF_4 & PL_LAB_CL_1 & $\begin{array}{c}\text { After Caustic Addition } \\
\text { to UFP-T01A } \\
\text { After Caustic Addition } \\
\text { to UFP-T02A }\end{array}$ & 702.83 & -- & 97.19 \\
S_02_AML_017_XX_0977_CUF_4 & PL_LAB_CL_2 & $\begin{array}{c}\text { Before Caustic } \\
\text { Addition to UFP-T01A }\end{array}$ & 538.30 & 156.40 & 105.30 \\
S_01AIM_020_XX_0748_CUF_4 & PL_LAB_CL_3 & $\begin{array}{c}\text { Before Caustic } \\
\text { Addition to UFP-T02A }\end{array}$ & 356.72 & 290.81 & 152.61 \\
\hline S_02AML_015_XX_0976_CUF_4 & PL_LAB_CL_4 & & & & \\
\hline
\end{tabular}


These tests were performed by placing the required amount of PEP simulant, $19 \mathrm{M} \mathrm{NaOH}$, and DI water in the test vessel, attaching the lid, and heating to $58^{\circ} \mathrm{C}$ for tests PL_LAB_CL_1 and PL_LAB_CL_3 and to $71^{\circ} \mathrm{C}$ for tests PL_LAB_CL_2 and PL_LAB_CL_4 while stirring at $120 \mathrm{rpm}$. After reaching the target temperature for each test, the vessel contents were heated at a linear rate to $98 \pm 2{ }^{\circ} \mathrm{C}$ over a 3 -hour time span. When the vessel reached $88 \pm 2{ }^{\circ} \mathrm{C}$, a sample was taken to represent the collection at $-10^{\circ} \mathrm{C}$ below the leaching temperature and was labeled as the "- $0.85 \mathrm{hr}$ " sample. At $98 \pm 2{ }^{\circ} \mathrm{C}$, the time zero $(0 \mathrm{hr})$ sample was taken, and the test was held for 24 hours at this temperature. Samples were taken at 1, 2, 4, 8, 10, 12, 14, 16, 20, 22, and 24 hours and immediately filtered through a $0.45-\mu \mathrm{m}$ syringe filter. These samples were then analyzed by ICP-AES for Al, Cr, and Na concentration at SWRI, and the results are shown in Table 6.2 through Table 6.4. Slurry samples were also taken at the beginning and end of the test. Some slurry samples were washed three times with $0.01 \mathrm{M} \mathrm{NaOH}$, and others were not washed. These samples were analyzed by ICP-AES for major cations at SWRI, and these results are shown in Table 6.5 through Table 6.8. The samples were also analyzed by ion chromatography (IC) for anion concentrations, which are shown in Table 6.9 through Table 6.12 for the supernate and Table 6.13 for the slurries. The density and wt\% solids of the initial slurry for each test were measured by SWRI using pycnometers for the density, and the slurry and supernate were dried for the wt $\%$ solids. These results are shown in Table 6.14 and Table 6.15. The $\mathrm{OH}^{-}$was titrated in the supernate samples by SWRI, and the results are shown in Table 6.16 in terms of milli-equivalents per gram (meq/g).

Table 6.2. Supernate Al Analysis from PEP Functional Test Caustic Leach

\begin{tabular}{cccccc}
\hline $\begin{array}{c}\text { Time } \\
(\mathrm{hrs})\end{array}$ & $\begin{array}{c}\text { Temp. } \\
\left({ }^{\circ} \mathrm{C}\right)\end{array}$ & $\begin{array}{c}\text { PL_LAB_CL_1 } \\
(\mathrm{mg} \text { A1/kg soln })\end{array}$ & $\begin{array}{c}\text { PL_LAB_CL_2 } \\
(\mathrm{mg} \mathrm{Al} / \mathrm{kg} \text { soln })\end{array}$ & $\begin{array}{c}\text { PL_LAB_CL_3 } \\
(\mathrm{mg} \text { A1/kg soln })\end{array}$ & $\begin{array}{c}\text { PL_LAB_CL_4 } \\
(\mathrm{mg} \text { A1/kg soln })\end{array}$ \\
\hline Initial & 25.1 & 4,640 & 11,100 & 2,370 & 3,940 \\
-0.85 & 88.1 & 7,120 & 12,100 & 6,720 & 12,000 \\
0 & 99.2 & 7,200 & 12,500 & 6,850 & 12,100 \\
1 & 99.1 & 7,150 & 12,800 & 6,990 & 12,800 \\
2 & 99.0 & 7,370 & 13,200 & 7,000 & 13,100 \\
4 & 99.0 & 7,670 & 14,100 & 7,280 & 13,900 \\
8 & 98.5 & 8,600 & 16,800 & 7,680 & No sample \\
10 & 98.9 & 8,800 & 16,300 & 8,020 & No sample \\
12 & 98.9 & 9,050 & 16,800 & 8,390 & 17,300 \\
14 & 98.9 & 9,600 & 17,500 & 8,640 & 17,700 \\
16 & 98.6 & 10,100 & 18,100 & 8,970 & 18,600 \\
18 & 99.0 & 10,400 & 19,000 & 9,420 & 19,100 \\
20 & 99.1 & 10,500 & 18,500 & 9,590 & 19,600 \\
22 & 99.1 & 10,800 & 19,800 & 9,870 & 20,100 \\
24 & 99.1 & 11,100 & 19,500 & 10,100 & 20,900 \\
\hline
\end{tabular}


Table 6.3. Supernate Cr Analysis from PEP Functional Test Caustic Leach

\begin{tabular}{cccccc}
\hline Time $(\mathrm{hrs})$ & $\begin{array}{c}\text { Temp. } \\
\left({ }^{\circ} \mathrm{C}\right)\end{array}$ & $\begin{array}{c}\text { PL_LAB_CL_1 } \\
(\mathrm{mg} \mathrm{Cr} / \mathrm{kg} \operatorname{soln})\end{array}$ & $\begin{array}{c}\text { PL_LAB_CL_2 } \\
(\mathrm{mg} \text { Cr/kg soln })\end{array}$ & $\begin{array}{c}\text { PL_LAB_CL_3 } \\
(\mathrm{mg} \text { Cr/kg soln })\end{array}$ & $\begin{array}{c}\text { PL_LAB_CL_4 } \\
(\mathrm{mg} \text { Cr/kg soln })\end{array}$ \\
\hline Initial & 25.1 & 1.16 & 1.54 & 1.10 & 0.85 \\
-0.85 & 88.1 & 1.26 & 2.22 & 1.19 & 1.36 \\
0 & 99.2 & 1.29 & 2.46 & 1.30 & 1.57 \\
1 & 99.1 & 1.30 & 2.55 & 1.36 & 1.67 \\
2 & 99.0 & 1.35 & 2.61 & 1.32 & 1.76 \\
4 & 99.0 & 1.38 & 2.77 & 1.38 & 1.88 \\
8 & 98.5 & 1.51 & 3.24 & 1.52 & No sample \\
10 & 98.9 & 1.49 & 3.01 & 1.50 & No sample \\
12 & 98.9 & 1.51 & 3.08 & 1.51 & 2.09 \\
14 & 98.9 & 1.62 & 3.14 & 1.48 & 2.10 \\
16 & 98.6 & 1.63 & 3.25 & 1.48 & 2.14 \\
18 & 99.0 & 1.65 & 3.38 & 1.59 & 2.21 \\
20 & 99.1 & 1.62 & 3.22 & 1.56 & 2.21 \\
22 & 99.1 & 1.69 & 3.37 & 1.69 & 2.26 \\
24 & 99.1 & 1.74 & 3.41 & 1.72 & 2.30 \\
\hline
\end{tabular}

Table 6.4. Supernate Na Analysis from PEP Functional Test Caustic Leach

\begin{tabular}{cccccc}
\hline Time $(\mathrm{hrs})$ & $\begin{array}{c}\text { Temp. } \\
\left({ }^{\circ} \mathrm{C}\right)\end{array}$ & $\begin{array}{c}\text { PL_LAB_CL_1 } \\
(\mathrm{mg} \mathrm{Na} / \mathrm{kg} \text { soln })\end{array}$ & $\begin{array}{l}\text { PL_LAB_CL_2 } \\
(\mathrm{mg} \mathrm{Na} / \mathrm{kg} \text { soln })\end{array}$ & $\begin{array}{l}\text { PL_LAB_CL_3 } \\
(\mathrm{mg} \text { Na/kg soln })\end{array}$ & $\begin{array}{c}\text { PL_LAB_CL_4 } \\
(\mathrm{mg} \mathrm{Na} / \mathrm{kg} \text { soln })\end{array}$ \\
\hline Initial & 25.1 & 131,000 & 149,000 & 129,000 & 159,000 \\
-0.85 & 88.1 & 133,000 & 160,000 & 124,000 & 151,000 \\
0 & 99.2 & 132,000 & 163,000 & 124,000 & 150,000 \\
1 & 99.1 & 130,000 & 163,000 & 123,000 & 151,000 \\
2 & 99.0 & 133,000 & 163,000 & 123,000 & 151,000 \\
4 & 99.0 & 132,000 & 164,000 & 124,000 & 152,000 \\
8 & 98.5 & 139,000 & 167,000 & 124,000 & No sample \\
10 & 98.9 & 132,000 & 165,000 & 124,000 & No sample \\
12 & 98.9 & 137,000 & 165,000 & 123,000 & 154,000 \\
14 & 98.9 & 142,000 & 167,000 & 127,000 & 153,000 \\
16 & 98.6 & 144,000 & 171,000 & 127,000 & 152,000 \\
18 & 99.0 & 145,000 & 174,000 & 132,000 & 154,000 \\
20 & 99.1 & 144,000 & 171,000 & 131,000 & 154,000 \\
22 & 99.1 & 145,000 & 174,000 & 132,000 & 156,000 \\
24 & 99.1 & 147,000 & 174,000 & 132,000 & 158,000 \\
\hline
\end{tabular}


Table 6.5. Slurry Analysis from PEP Functional Test Caustic Leach Test 1

\begin{tabular}{|c|c|c|c|c|c|}
\hline & $\begin{array}{l}\text { A_LAB_- } \\
\text { CL_1_IN_SL } \\
\text { (mg/kg slurry) }\end{array}$ & $\begin{array}{c}\text { A_LAB_- } \\
\text { CL_1_IS_SL } \\
\text { (mg/kg slurry) }\end{array}$ & $\begin{array}{l}\text { A_LAB_- } \\
\text { CL_1_FS1_SL } \\
\text { (mg/kg slurry) }\end{array}$ & $\begin{array}{l}\text { A_LAB_- } \\
\text { CL_1_FS2_SL } \\
\text { (mg/kg slurry) }\end{array}$ & $\begin{array}{l}\text { A_LAB_ } \\
\text { CL_1_FS3_SL } \\
\text { (mg/kg slurry) }\end{array}$ \\
\hline $\begin{array}{c}\text { Sample } \\
\text { Description }\end{array}$ & $\begin{array}{c}\text { Test } 1 \text { Initial } \\
\text { Unwashed } \\
\text { Slurry }\end{array}$ & $\begin{array}{c}\text { Test } 1 \text { Initial } \\
\text { Washed Slurry }\end{array}$ & $\begin{array}{c}\text { Test } 1 \text { Final } \\
\text { Washed Slurry }-1\end{array}$ & $\begin{array}{c}\text { Test } 1 \text { Final } \\
\text { Washed Slurry }-2\end{array}$ & $\begin{array}{c}\text { Test } 1 \text { Final } \\
\text { Washed Slurry -3 }\end{array}$ \\
\hline $\mathrm{Al}$ & 9,850 & 27,200 & 15,100 & 16,900 & 39,000 \\
\hline $\mathrm{Ca}$ & 96.9 & 511 & 653 & 675 & 648 \\
\hline $\mathrm{Cr}$ & 1.66 & 3.08 & 2.17 & 2.08 & 15.5 \\
\hline $\mathrm{Fe}$ & 3,070 & 16,400 & 20,300 & 20,800 & 20,200 \\
\hline $\mathrm{Mg}$ & 65.0 & 348 & 434 & 440 & 432 \\
\hline $\mathrm{Mn}$ & 671 & 3,450 & 4,180 & 4,230 & 4,100 \\
\hline $\mathrm{Ni}$ & 93.6 & 507 & 629 & 644 & 629 \\
\hline $\mathrm{Na}$ & 125,000 & 901 & 1,270 & 1,400 & 1,290 \\
\hline $\mathrm{P}$ & 1,280 & 146 & 202 & 231 & 255 \\
\hline $\mathrm{Sr}$ & 27.2 & 138 & 168 & 173 & 169 \\
\hline $\mathrm{Zr}$ & 82.4 & 384 & 458 & 466 & 524 \\
\hline
\end{tabular}

Table 6.6. Slurry Analysis from PEP Functional Test Caustic Leach Test 2

\begin{tabular}{|c|c|c|c|c|c|}
\hline & $\begin{array}{l}\text { A_LAB_- } \\
\text { CL_2_IN_SL } \\
\text { (mg/kg slurry) }\end{array}$ & $\begin{array}{l}\text { A_LAB_- } \\
\text { CL_2_IS_SL } \\
\text { (mg/kg slurry) }\end{array}$ & $\begin{array}{l}\text { A_LAB_- } \\
\text { CL_2_FS1_SL } \\
\text { (mg/kg slurry) }\end{array}$ & $\begin{array}{l}\text { A_LAB_- } \\
\text { CL_2_FS2_SL } \\
\text { (mg/kg slurry) }\end{array}$ & $\begin{array}{l}\text { A_LAB_ } \\
\text { CL_2_FS3_SL } \\
\text { (mg/kg slurry) }\end{array}$ \\
\hline $\begin{array}{c}\text { Sample } \\
\text { Description }\end{array}$ & $\begin{array}{c}\text { Test } 2 \text { Initial } \\
\text { Unwashed } \\
\text { Slurry }\end{array}$ & $\begin{array}{c}\text { Test } 2 \text { Initial } \\
\text { Washed Slurry }\end{array}$ & $\begin{array}{c}\text { Test } 2 \text { Final } \\
\text { Washed Slurry }-1\end{array}$ & $\begin{array}{c}\text { Test } 2 \text { Final Washed } \\
\text { Slurry }-2\end{array}$ & $\begin{array}{c}\text { Test } 2 \text { Final } \\
\text { Washed Slurry }-3\end{array}$ \\
\hline $\mathrm{Al}$ & 19,700 & 22,500 & 37,000 & 41,200 & 42,400 \\
\hline $\mathrm{Ca}$ & 229 & 1,030 & 1,220 & 1,230 & 1,250 \\
\hline $\mathrm{Cr}$ & 3.20 & 17.0 & 32.9 & 33.4 & 33.8 \\
\hline $\mathrm{Fe}$ & 7,260 & 32,700 & 38,600 & 38,900 & 39,600 \\
\hline $\mathrm{Mg}$ & 155 & 708 & 811 & 821 & 834 \\
\hline $\mathrm{Mn}$ & 1,630 & 6,630 & 7,750 & 7,800 & 8,070 \\
\hline $\mathrm{Ni}$ & 221 & 1,010 & 1,200 & 1,210 & 1,240 \\
\hline $\mathrm{Na}$ & 141,000 & 2,050 & 3,060 & 3,090 & 3,240 \\
\hline $\mathrm{P}$ & 756 & 101 & 217 & 175 & 199 \\
\hline $\mathrm{Sr}$ & 64.5 & 274 & 325 & 322 & 329 \\
\hline $\mathrm{Zr}$ & 196 & 695 & 712 & 371 & 323 \\
\hline
\end{tabular}


Table 6.7. Slurry Analysis from PEP Functional Test Caustic Leach Test 3

\begin{tabular}{|c|c|c|c|c|c|}
\hline & $\begin{array}{l}\text { A_LAB_- } \\
\text { CL_3_IN_SL } \\
\text { (mg/kg slurry) }\end{array}$ & $\begin{array}{c}\text { A_LAB_- } \\
\text { CL_3_IS_SL } \\
\text { (mg/kg slurry) }\end{array}$ & $\begin{array}{l}\text { A_LAB_- } \\
\text { CL_3_FS1_SL } \\
\text { (mg/kg slurry) }\end{array}$ & $\begin{array}{l}\text { A_LAB_- } \\
\text { CL_3_FS2_SL } \\
\text { (mg/kg slurry) }\end{array}$ & $\begin{array}{l}\text { A_LAB_- } \\
\text { CL_3_FS3_SL } \\
\text { (mg/kg slurry) }\end{array}$ \\
\hline $\begin{array}{c}\text { Sample } \\
\text { Description }\end{array}$ & $\begin{array}{c}\text { Test } 3 \text { Initial } \\
\text { Unwashed } \\
\text { Slurry }\end{array}$ & $\begin{array}{c}\text { Test } 3 \text { Initial } \\
\text { Washed Slurry }\end{array}$ & $\begin{array}{c}\text { Test } 3 \text { Final } \\
\text { Washed Slurry }-1\end{array}$ & $\begin{array}{c}\text { Test } 3 \text { Final } \\
\text { Washed Slurry }-2\end{array}$ & $\begin{array}{c}\text { Test } 3 \text { Final } \\
\text { Washed Slurry }-3\end{array}$ \\
\hline $\mathrm{Al}$ & 8,790 & 37,500 & 17,700 & 18,100 & 17,100 \\
\hline $\mathrm{Ca}$ & 98.1 & 491 & 629 & 635 & 635 \\
\hline $\mathrm{Cr}$ & 1.52 & 3.20 & 2.18 & 2.05 & 2.00 \\
\hline $\mathrm{Fe}$ & 3,050 & 15,600 & 19,700 & 20,000 & 20,000 \\
\hline $\mathrm{Mg}$ & 65.5 & 339 & 422 & 435 & 418 \\
\hline $\mathrm{Mn}$ & 678 & 3,300 & 4,060 & 4,080 & 4,070 \\
\hline $\mathrm{Ni}$ & 94.2 & 484 & 608 & 620 & 624 \\
\hline $\mathrm{Na}$ & 122,000 & 823 & 1,370 & 1,220 & 1,190 \\
\hline $\mathrm{P}$ & 714 & 79.1 & 298 & 183 & 195 \\
\hline $\mathrm{Sr}$ & 27.2 & 133 & 166 & 171 & 167 \\
\hline $\mathrm{Zr}$ & 68.9 & 409 & 429 & 472 & 469 \\
\hline
\end{tabular}

Table 6.8. Slurry Analysis from PEP Functional Test Caustic Leach Test 4

\begin{tabular}{|c|c|c|c|c|c|}
\hline & $\begin{array}{l}\text { A_LAB_- } \\
\text { CL_4_IN_SL } \\
\text { (mg/kg slurry) }\end{array}$ & $\begin{array}{l}\text { A_LAB_- } \\
\text { CL_4_IS_SL } \\
\text { (mg/kg slurry) }\end{array}$ & $\begin{array}{l}\text { A_LAB_- } \\
\text { CL_4_FS1_SL } \\
\text { (mg/kg slurry) }\end{array}$ & $\begin{array}{l}\text { A_LAB_- } \\
\text { CL_4_FS2_SL } \\
\text { (mg/kg slurry) }\end{array}$ & $\begin{array}{c}\text { A_LAB_ } \\
\text { CL_4_FS3_SL } \\
\text { (mg/kg slurry) }\end{array}$ \\
\hline $\begin{array}{c}\text { Sample } \\
\text { Description }\end{array}$ & $\begin{array}{c}\text { Test } 4 \text { Initial } \\
\text { Unwashed } \\
\text { Slurry }\end{array}$ & $\begin{array}{c}\text { Test } 4 \text { Initial } \\
\text { Washed Slurry }\end{array}$ & $\begin{array}{c}\text { Test } 4 \text { Final } \\
\text { Washed Slurry }-1\end{array}$ & $\begin{array}{c}\text { Test } 4 \text { Final } \\
\text { Washed Slurry }-2\end{array}$ & $\begin{array}{c}\text { Test } 4 \text { Final } \\
\text { Washed Slurry }-3\end{array}$ \\
\hline $\mathrm{Al}$ & 20,000 & 60,200 & 32,000 & 31,100 & 31,400 \\
\hline $\mathrm{Ca}$ & 222 & 857 & 1,200 & 1,210 & 1,180 \\
\hline $\mathrm{Cr}$ & 2.53 & 27.8 & 32.9 & 30.6 & 31.0 \\
\hline $\mathrm{Fe}$ & 6,850 & 26,800 & 38,500 & 38,500 & 38,000 \\
\hline $\mathrm{Mg}$ & 151 & 579 & 801 & 818 & 801 \\
\hline $\mathrm{Mn}$ & 1,560 & 5,590 & 7,750 & 7,750 & 7,680 \\
\hline $\mathrm{Ni}$ & 220 & 830 & 1,210 & 1,200 & 1,190 \\
\hline $\mathrm{Na}$ & 146,000 & 1,740 & 2,900 & 3,010 & 2,910 \\
\hline $\mathrm{P}$ & 448 & 74.2 & 207 & 247 & 173 \\
\hline $\mathrm{Sr}$ & 62.0 & 228 & 320 & 321 & 312 \\
\hline $\mathrm{Zr}$ & 187 & 703 & 633 & 683 & 504 \\
\hline
\end{tabular}


Table 6.9. Anion Analysis of PL_LAB_CL_1 Supernate

\begin{tabular}{cccccccc}
\hline Time $(\mathrm{hrs})$ & $\begin{array}{c}\text { Temp. } \\
\left({ }^{\circ} \mathrm{C}\right)\end{array}$ & $\begin{array}{c}\text { Chloride } \\
(\mathrm{mg} / \mathrm{kg} \\
\text { soln })\end{array}$ & $\begin{array}{c}\text { Nitrate } \\
(\mathrm{mg} / \mathrm{kg} \\
\text { soln })\end{array}$ & $\begin{array}{c}\text { Nitrite } \\
(\mathrm{mg} / \mathrm{kg} \\
\text { soln) }\end{array}$ & $\begin{array}{c}\text { Oxalate } \\
(\mathrm{mg} / \mathrm{kg} \\
\text { soln })\end{array}$ & $\begin{array}{c}\text { Phosphate } \\
(\mathrm{mg} / \mathrm{kg} \text { soln })\end{array}$ & $\begin{array}{c}\text { Sulfate } \\
(\mathrm{mg} / \mathrm{kg} \\
\text { soln})\end{array}$ \\
\hline Initial & 25.1 & 964 & 58,000 & 13,100 & 164 & 2,030 & 10,200 \\
-0.85 & 88.1 & 810 & 60,700 & 13,400 & 197 & 4,110 & 10,400 \\
0 & 99.2 & 836 & 62,400 & 13,900 & 192 & 4,170 & 10,600 \\
1 & 99.1 & 811 & 60,200 & 13,400 & 189 & 4,050 & 10,400 \\
2 & 99.0 & 835 & 61,600 & 13,600 & 176 & 4,080 & 10,400 \\
4 & 99.0 & 869 & 63,300 & 14,000 & 173 & 4,170 & 10,700 \\
8 & 98.5 & 858 & 63,300 & 14,100 & 159 & 4,200 & 10,800 \\
10 & 98.9 & 865 & 63,300 & 14,100 & 152 & 4,200 & 10,800 \\
\hline 12 & 98.9 & 860 & 63,300 & 14,200 & 164 & 4,200 & 10,800 \\
14 & 98.9 & 875 & 64,700 & 14,400 & 149 & 4,260 & 10,800 \\
16 & 98.6 & 898 & 66,000 & 14,700 & 141 & 4,320 & 11,100 \\
18 & 99.0 & 901 & 66,900 & 14,900 & 137 & 4,380 & 11,100 \\
20 & 99.1 & 928 & 67,300 & 15,000 & 170 & 4,410 & 11,100 \\
22 & 99.1 & 929 & 68,600 & 15,200 & 145 & 4,470 & 11,200 \\
\hline 24 & 99.1 & 952 & 69,500 & 15,500 & 150 & 4,440 & 11,300 \\
\hline
\end{tabular}

Table 6.10. Anion Analysis of PL_LAB_CL_2 Supernate

\begin{tabular}{cccccccc}
\hline Time $(\mathrm{hrs})$ & $\begin{array}{c}\text { Temp. } \\
\left({ }^{\circ} \mathrm{C}\right)\end{array}$ & $\begin{array}{c}\text { Chloride } \\
(\mathrm{mg} / \mathrm{kg} \\
\text { soln })\end{array}$ & $\begin{array}{c}\text { Nitrate } \\
(\mathrm{mg} / \mathrm{kg} \\
\text { soln })\end{array}$ & $\begin{array}{c}\text { Nitrite } \\
(\mathrm{mg} / \mathrm{kg} \\
\text { soln })\end{array}$ & $\begin{array}{c}\text { Oxalate } \\
(\mathrm{mg} / \mathrm{kg} \\
\text { soln })\end{array}$ & $\begin{array}{c}\text { Shosphate } \\
(\mathrm{mg} / \mathrm{kg} \text { soln })\end{array}$ & $\begin{array}{c}\text { Sulfate } \\
(\mathrm{mg} / \mathrm{kg} \\
\text { soln })\end{array}$ \\
\hline Initial & 25.1 & 666 & 37,700 & 8,670 & 71.7 & 1,410 & 7,030 \\
-0.85 & 88.1 & 525 & 39,200 & 8,900 & 135 & 2,790 & 7,330 \\
0 & 99.2 & 510 & 39,100 & 9,000 & 109 & 2,750 & 7,230 \\
1 & 99.1 & 538 & 39,600 & 9,070 & 94.4 & 2,860 & 7,330 \\
2 & 99.0 & 525 & 39,400 & 9,040 & 94.2 & 2,790 & 7,430 \\
4 & 99.0 & 537 & 39,900 & 9,070 & 108 & 1,080 & 7,370 \\
8 & 98.5 & 552 & 40,500 & 9,920 & 73.4 & 2,810 & 7,810 \\
10 & 98.9 & 529 & 39,900 & 9,170 & 85.4 & 2,680 & 7,420 \\
12 & 98.9 & 522 & 39,900 & 9,230 & 79.0 & 2,700 & 7,510 \\
14 & 98.9 & 544 & 40,500 & 9,360 & 84.2 & 2,610 & 7,570 \\
16 & 98.6 & 558 & 41,200 & 9,430 & 75.5 & 2,720 & 7,620 \\
18 & 99.0 & 532 & 39,300 & 9,330 & 63.6 & 2,220 & 7,510 \\
20 & 99.1 & 566 & 42,200 & 9,590 & 78.0 & 2,690 & 7,770 \\
22 & 99.1 & 564 & 42,100 & 9,630 & 79.3 & 1,020 & 7,810 \\
\hline 24 & 99.1 & 549 & 40,800 & 9,300 & 76.8 & 2,590 & 7,510 \\
\hline
\end{tabular}


Table 6.11. Anion Analysis of PL_LAB_CL_3 Supernate

\begin{tabular}{cccccccc}
\hline $\begin{array}{c}\text { Time } \\
(\mathrm{hrs})\end{array}$ & $\begin{array}{c}\text { Temp. } \\
\left({ }^{\circ} \mathrm{C}\right)\end{array}$ & $\begin{array}{c}\text { Chloride } \\
(\mathrm{mg} / \mathrm{kg} \text { soln })\end{array}$ & $\begin{array}{c}\text { Nitrate } \\
(\mathrm{mg} / \mathrm{kg} \text { soln })\end{array}$ & $\begin{array}{c}\text { Nitrite } \\
(\mathrm{mg} / \mathrm{kg} \text { soln })\end{array}$ & $\begin{array}{c}\text { Oxalate } \\
(\mathrm{mg} / \mathrm{kg} \\
\text { soln })\end{array}$ & $\begin{array}{c}\text { Phosphate } \\
(\mathrm{mg} / \mathrm{kg} \mathrm{soln})\end{array}$ & $\begin{array}{c}\text { Sulfate } \\
(\mathrm{mg} / \mathrm{kg} \\
\text { soln })\end{array}$ \\
\hline Initial & 25.1 & 978 & 60,200 & 13,400 & 264 & 1,960 & 10,400 \\
-0.85 & 88.1 & 819 & 58,500 & 13,000 & 213 & 3,980 & 10,100 \\
0 & 99.2 & 805 & 57,600 & 12,900 & 203 & 3,800 & 9,960 \\
1 & 99.1 & 830 & 59,300 & 13,300 & 205 & 1,760 & 10,400 \\
2 & 99.0 & 829 & 58,500 & 13,000 & 208 & 3,950 & 10,100 \\
4 & 99.0 & 819 & 58,500 & 13,100 & 209 & 3,920 & 10,200 \\
8 & 98.5 & 818 & 58,900 & 13,200 & 242 & 3,920 & 10,300 \\
10 & 98.9 & 819 & 58,900 & 13,200 & 195 & 3,920 & 10,200 \\
12 & 98.9 & 837 & 58,900 & 13,200 & 195 & 3,950 & 10,300 \\
14 & 98.9 & 820 & 58,500 & 13,200 & 183 & 3,920 & 10,100 \\
16 & 98.6 & 841 & 59,300 & 13,300 & 194 & 2,820 & 10,300 \\
18 & 99.0 & 875 & 62,000 & 14,000 & 156 & 4,140 & 10,700 \\
20 & 99.1 & 898 & 64,200 & 14,200 & 169 & 4,230 & 10,900 \\
22 & 99.1 & 897 & 62,900 & 14,100 & 162 & 4,200 & 10,700 \\
24 & 99.1 & 892 & 63,300 & 14,100 & 160 & 4,170 & 10,800 \\
\hline
\end{tabular}

Table 6.12. Anion Analysis of PL_LAB_CL_4 Supernate

\begin{tabular}{cccccccc}
\hline $\begin{array}{c}\text { Time } \\
(\mathrm{hrs})\end{array}$ & $\begin{array}{c}\text { Temp. } \\
\left({ }^{\circ} \mathrm{C}\right)\end{array}$ & $\begin{array}{c}\text { Chloride } \\
(\mathrm{mg} / \mathrm{kg} \text { soln })\end{array}$ & $\begin{array}{c}\text { Nitrate } \\
(\mathrm{mg} / \mathrm{kg} \text { soln })\end{array}$ & $\begin{array}{c}\text { Nitrite } \\
(\mathrm{mg} / \mathrm{kg} \text { soln })\end{array}$ & $\begin{array}{c}\text { Oxalate } \\
(\mathrm{mg} / \mathrm{kg} \text { soln })\end{array}$ & $\begin{array}{c}\text { Phosphate } \\
(\mathrm{mg} / \mathrm{kg} \text { soln })\end{array}$ & $\begin{array}{c}\text { Sulfate } \\
(\mathrm{mg} / \mathrm{kg} \text { soln })\end{array}$ \\
\hline Initial & 25.1 & 632 & 33,000 & 7,720 & 74.4 & 1,250 & 6,020 \\
-0.85 & 88.1 & 446 & 32,700 & 7,560 & 72.6 & 2,330 & 5,990 \\
0 & 99.2 & 453 & 32,900 & 7,590 & 84.4 & 2,360 & 5,850 \\
1 & 99.1 & 454 & 32,900 & 7,560 & 81.1 & 1,010 & 5,940 \\
2 & 99.0 & 467 & 33,600 & 7,690 & 81.7 & 2,290 & 6,020 \\
4 & 99.0 & 454 & 33,100 & 7,690 & 92.0 & 2,220 & 6,000 \\
12 & 98.9 & 467 & 33,300 & 7,750 & 71.0 & 2,170 & 5,950 \\
14 & 98.9 & 460 & 33,100 & 7,750 & 68.1 & 1,720 & 5,890 \\
16 & 98.6 & 453 & 32,800 & 7,660 & 64.2 & 2,090 & 5,860 \\
18 & 99.0 & 460 & 33,200 & 7,720 & 69.4 & 2,090 & 5,900 \\
20 & 99.1 & 470 & 33,800 & 7,790 & 66.8 & 2,110 & 6,000 \\
22 & 99.1 & 477 & 34,300 & 7,850 & 64.4 & 2,070 & 6,080 \\
24 & 99.1 & 477 & 34,100 & 7,890 & 58.8 & 1,980 & 6,060 \\
\hline
\end{tabular}


Table 6.13. Anion Analysis of Slurry Samples

\begin{tabular}{|c|c|c|c|c|c|c|c|}
\hline Sample ID & $\begin{array}{c}\text { Sample } \\
\text { Description }\end{array}$ & $\begin{array}{c}\text { Chloride } \\
\text { (mg/kg } \\
\text { slurry) }\end{array}$ & $\begin{array}{l}\text { Nitrate } \\
\text { (mg/kg } \\
\text { slurry) }\end{array}$ & $\begin{array}{l}\text { Nitrite } \\
\text { (mg/kg } \\
\text { slurry) }\end{array}$ & $\begin{array}{l}\begin{array}{l}\text { Oxalate } \\
\text { (mg/kg } \\
\text { slurry) }\end{array}\end{array}$ & $\begin{array}{c}\text { Phosphate } \\
\text { (mg/kg slurry) }\end{array}$ & $\begin{array}{l}\text { Sulfate } \\
\text { (mg/kg } \\
\text { slurry) }\end{array}$ \\
\hline A_LAB_CL_1_IS_SL & $\begin{array}{c}\text { Test } 1 \text { Initial } \\
\text { Washed Slurry }\end{array}$ & 21.7 & $<87$ & $<65$ & $<19.7$ & 108 & $<19.7$ \\
\hline PL_LAB_CL_1_IN_SL & $\begin{array}{l}\text { Test } 1 \text { Initial } \\
\text { Slurry -1 }\end{array}$ & 673 & 50,000 & 11,100 & 2,950 & 3,460 & 8,660 \\
\hline A_LAB_CL_1_FS1_SL & $\begin{array}{c}\text { Test } 1 \text { Final } \\
\text { Washed Slurry }-1\end{array}$ & $<18.9$ & $<84$ & $<62$ & $<18.9$ & 316 & $<18.9$ \\
\hline A_LAB_CL_1_FS2_SL & $\begin{array}{c}\text { Test } 1 \text { Final } \\
\text { Washed Slurry -2 }\end{array}$ & 53.5 & $<85$ & $<63$ & 25.5 & 383 & $<19.3$ \\
\hline A_LAB_CL_1_FS3_SL & $\begin{array}{c}\text { Test } 1 \text { Final } \\
\text { Washed Slurry -3 }\end{array}$ & 36.7 & 87 & $<63$ & $<19.1$ & 325 & $<19.1$ \\
\hline A_LAB_CL_2_IS_SL & $\begin{array}{c}\text { Test } 2 \text { Initial } \\
\text { Washed Slurry }\end{array}$ & 21.9 & 83 & $<56$ & 66.3 & 157 & $<17.1$ \\
\hline PL_LAB_CL_2_IN_SL & $\begin{array}{l}\text { Test } 2 \text { Initial } \\
\text { Slurry }-1\end{array}$ & 427 & 32,400 & 7,390 & 6,270 & 2,180 & 5,950 \\
\hline A_LAB_CL_2_FS1_SL & $\begin{array}{c}\text { Test } 2 \text { Final } \\
\text { Washed Slurry }-1\end{array}$ & 64.7 & 103 & $<62$ & 219 & 561 & 21.4 \\
\hline A_LAB_CL_2_FS2_SL & $\begin{array}{c}\text { Test } 2 \text { Final } \\
\text { Washed Slurry -2 }\end{array}$ & $<18.4$ & 91 & $<60$ & 163 & 907 & $<18.4$ \\
\hline A_LAB_CL_2_FS3_SL & $\begin{array}{c}\text { Test } 2 \text { Final } \\
\text { Washed Slurry -3 }\end{array}$ & 25.9 & 96 & $<59$ & 156 & 913 & $<17.9$ \\
\hline PL_LAB_CL_3_IN_SL & $\begin{array}{l}\text { Test } 3 \text { Initial } \\
\text { Slurry -1 }\end{array}$ & 720 & 52,300 & 11,600 & 3,120 & 1,920 & 9,030 \\
\hline A_LAB_CL_3_IS_SL & $\begin{array}{l}\text { Test } 3 \text { Initial } \\
\text { Slurry }-2\end{array}$ & $<18.3$ & $<81$ & $<60$ & $<18.3$ & $<56$ & $<18.3$ \\
\hline A_LAB_CL_3_FS1_SL & $\begin{array}{c}\text { Test } 3 \text { Final } \\
\text { Washed Slurry -1 }\end{array}$ & 35.4 & $<84$ & $<62$ & 21.3 & 573 & $<19.0$ \\
\hline A_LAB_CL_3_FS2_SL & $\begin{array}{c}\text { Test } 3 \text { Final } \\
\text { Washed Slurry -2 }\end{array}$ & 20.5 & $<85$ & $<63$ & 32.8 & 283 & $<19.3$ \\
\hline A_LAB_CL_3_FS3_SL & $\begin{array}{c}\text { Test } 3 \text { Final } \\
\text { Washed Slurry -3 }\end{array}$ & 27.2 & $<84$ & $<62$ & 23.5 & 276 & $<19.0$ \\
\hline PL_LAB_CL_4_IN_SL & $\begin{array}{l}\text { Test } 4 \text { Initial } \\
\text { Slurry -1 }\end{array}$ & 389 & 28,400 & 6,510 & 5,950 & 1,840 & 5,100 \\
\hline A_LAB_CL_4_IS_SL & $\begin{array}{l}\text { Test } 4 \text { Initial } \\
\text { Slurry -2 }\end{array}$ & 93.3 & 86 & $<57$ & 103 & $<53$ & 20.0 \\
\hline A_LAB_CL_4_FS1_SL & $\begin{array}{c}\text { Test } 4 \text { Final } \\
\text { Washed Slurry -1 }\end{array}$ & 89.6 & 90 & $<61$ & 180 & 591 & $<18.7$ \\
\hline A_LAB_CL_4_FS2_SL & $\begin{array}{c}\text { Test } 4 \text { Final } \\
\text { Washed Slurry -2 }\end{array}$ & 33.6 & 92 & $<60$ & 174 & 598 & $<18.2$ \\
\hline A_LAB_CL_4_FS3_SL & $\begin{array}{c}\text { Test } 4 \text { Final } \\
\text { Washed Slurry -3 }\end{array}$ & 21.8 & 91 & $<62$ & 173 & 598 & $<18.8$ \\
\hline
\end{tabular}


Table 6.14. PEP Parallel Functional Test Density

\begin{tabular}{lcc}
\hline \multicolumn{1}{c}{ Sample ID } & Sample Description & Density $(\mathrm{g} / \mathrm{mL})$ \\
\hline PL-LAB_CL_1_IN_SUP & Test 1 Initial Supernate & 1.274 \\
PL-LAB_CL_2_IN_SUP & Test 2 Initial Supernate & 1.271 \\
PL-LAB_CL_3_IN_SUP & Test 3 Initial Supernate & 1.252 \\
PL-LAB_CL_4_IN_SUP & Test 4 Initial Supernate & 1.326 \\
PL-LAB_CL_1_IN_SL & Test 1 Initial Slurry & 1.304 \\
PL-LAB_CL_2_IN_SL & Test 2 Initial Slurry & 1.362 \\
PL-LAB_CL_3_IN_SL & Test 3 Initial Slurry & 1.302 \\
PL-LAB_CL_4_IN_SL & Test 4 Initial Slurry & 1.374 \\
\hline
\end{tabular}

Table 6.15. PEP Parallel Functional Test Physical Properties of Initial Slurries

\begin{tabular}{|c|c|c|c|c|}
\hline Property & $\begin{array}{c}\text { PL_LAB_CL } \\
11 \text { IN_SL }\end{array}$ & $\begin{array}{l}\text { PL_LAB_CL } \\
\text { ___IN_SL }\end{array}$ & $\begin{array}{l}\text { PL_LAB_CL } \\
\text { 3_IN_SL }\end{array}$ & $\begin{array}{c}\text { PL_LAB_CL } \\
4 \text { _IN_SL }\end{array}$ \\
\hline Sample Description & $\begin{array}{l}\text { Test } 1 \text { Initial } \\
\text { Slurry }\end{array}$ & $\begin{array}{l}\text { Test } 2 \text { Initial } \\
\text { Slurry }\end{array}$ & $\begin{array}{c}\text { Test } 3 \text { Initial } \\
\text { Slurry }\end{array}$ & $\begin{array}{l}\text { Test } 4 \text { Initial } \\
\text { Slurry }\end{array}$ \\
\hline Bulk Density $(\mathrm{g} / \mathrm{mL})$ & 1.304 & 1.355 & 1.294 & 1.355 \\
\hline Density of Cent. Solids $(\mathrm{g} / \mathrm{mL})$ & 1.823 & 1.802 & 2.056 & 1.841 \\
\hline Vol\% of Cent. Solids & 10.0 & 15.0 & 7.50 & 15.0 \\
\hline Wt $\%$ Cent. Solids & 14.0 & 20.0 & 11.9 & 20.4 \\
\hline Supernate Density $(\mathrm{g} / \mathrm{mL})$ & 1.240 & 1.268 & 1.225 & 1.261 \\
\hline $\mathrm{Wt} \%$ Total Solids & 31.8 & 36.9 & 31.4 & 37.5 \\
\hline Wt $\%$ Oven Dried Solids & 42.7 & 50.6 & 45.7 & 50.6 \\
\hline $\mathrm{Wt} \%$ UDS & 2.49 & 5.08 & 2.73 & 5.04 \\
\hline $\mathrm{Wt} \%$ Dissolved Solids & 30.2 & 33.7 & 29.6 & 34.4 \\
\hline $\mathrm{Wt} \%$ Supernate Liquid & 85.6 & 79.6 & 87.6 & 79.1 \\
\hline
\end{tabular}


Table 6.16. PEP Parallel Functional Test Free OH Concentration

\begin{tabular}{|c|c|c|c|}
\hline Sample ID & $\begin{array}{c}\text { OH Conc. } \\
\text { (mol/kg soln) }\end{array}$ & Sample ID & $\begin{array}{c}\text { OH Conc. } \\
\text { (mol/kg soln) }\end{array}$ \\
\hline PL-LAB_CL_1_IN_SUP & 3.46 & PL-LAB_CL_3_IN_SUP & 3.45 \\
\hline PL-LAB_CL_1_010C & 3.50 & PL-LAB_CL_3_010C & 3.18 \\
\hline PL-LAB_CL_1_000 & 3.55 & PL-LAB_CL_3_000 & 3.22 \\
\hline PL-LAB_CL_1_001 & 3.54 & PL-LAB_CL_3_001 & 3.24 \\
\hline PL-LAB_CL_1_002 & 3.52 & PL-LAB_CL_3_002 & 3.19 \\
\hline PL-LAB_CL_1_004 & 3.63 & PL-LAB_CL_3_004 & 3.20 \\
\hline PL-LAB_CL_1_008 & 3.63 & PL-LAB_CL_3_008 & 3.16 \\
\hline PL-LAB_CL_1_010 & 3.61 & PL-LAB_CL_3_010 & 3.19 \\
\hline PL-LAB_CL_1_012 & 3.63 & PL-LAB_CL_3_012 & 3.21 \\
\hline PL-LAB_CL_1_014 & 3.69 & PL-LAB_CL_3_014 & 3.24 \\
\hline PL-LAB_CL_1_016 & 3.79 & PL-LAB_CL_3_016 & 3.17 \\
\hline PL-LAB_CL_1_018 & 3.77 & PL-LAB_CL_3_018 & 3.35 \\
\hline PL-LAB_CL_1_020 & 3.71 & PL-LAB_CL_3_020 & 3.27 \\
\hline PL-LAB_CL_1_022 & 3.75 & PL-LAB_CL_3_022 & 3.30 \\
\hline PL-LAB_CL_1_024 & 3.77 & PL-LAB_CL_3_024 & 3.26 \\
\hline PL-LAB_CL_2_IN_SUP & 4.55 & PL-LAB_CL_4_IN_SUP & 5.37 \\
\hline PL-LAB_CL_2_010C & 4.77 & PL-LAB_CL_4_010C & 4.95 \\
\hline PL-LAB_CL_2_000 & 4.81 & PL-LAB_CL_4_000 & 4.93 \\
\hline PL-LAB_CL_2_001 & 4.79 & PL-LAB_CL_4_001 & 4.94 \\
\hline PL-LAB_CL_2_002 & 4.82 & PL-LAB_CL_4_002 & 4.93 \\
\hline PL-LAB_CL_2_004 & 4.74 & PL-LAB_CL_4_004 & 4.87 \\
\hline PL-LAB_CL_2_008 & 5.14 & PL-LAB_CL_4_008 & NM \\
\hline PL-LAB_CL_2_010 & 4.74 & PL-LAB_CL_4_010 & NM \\
\hline PL-LAB_CL_2_012 & 4.81 & PL-LAB_CL_4_012 & 4.86 \\
\hline PL-LAB_CL_2_014 & 4.82 & PL-LAB_CL_4_014 & 4.82 \\
\hline PL-LAB_CL_2_016 & 4.81 & PL-LAB_CL_4_016 & 4.68 \\
\hline PL-LAB_CL_2_018 & 5.01 & PL-LAB_CL_4_018 & 4.79 \\
\hline PL-LAB_CL_2_020 & 4.80 & PL-LAB_CL_4_020 & NM \\
\hline PL-LAB_CL_2_022 & 4.81 & PL-LAB_CL_4_022 & 4.77 \\
\hline PL-LAB_CL_2_024 & 4.74 & PL-LAB_CL_4_024 & NM \\
\hline $\mathrm{NM}=$ not measured & & & \\
\hline
\end{tabular}

\subsection{PEP Test A Caustic Leach}

In PEP Test A, which was conducted under Test Instruction TI-WTP-PEP-065 (TI-065), simulant stored in HLP-VSL-T22 was transferred into vessel UFP-VSL-T01A together with caustic reagent (nominally $19 \mathrm{M} \mathrm{NaOH}$ ) that was injected into the feed transfer line. A sample of the feed slurry (without added caustic) was taken by grab sample from HLP-VSL-T22 for use in the laboratory-scale testing (A_LAB_CL_2). This slurry sample was taken on January 31, 2009 at 9:53. 
An AFA, Dow Corning Q2-3183A, was added directly to UFP-VSL-T01A in a quantity expected to produce a nominal concentration of $350 \mathrm{ppm}$ in the slurry. After this addition, another sample of slurry (composed of feed plus caustic and AFA) was taken from the inner CD sample loop at the middle-elevation port in UFP-VSL-T01A for use as feed in the laboratory-scale caustic leach tests (A_LAB_CL_1). This slurry sample was taken on January 31, 2009 at 13:30. The sample was rapidly cooled in a cold water bath to ambient temperature to minimize any further leaching reaction.

The laboratory-scale feed was stored at laboratory ambient temperature (approximately $20^{\circ} \mathrm{C}$ to $25^{\circ} \mathrm{C}$ ) until it was used. The delay between the time when the feed was acquired from PEP and the time laboratory-scale testing started was about 9 days (from January 31, 2009 to February 9, 2009).

The PEP sample ID, laboratory sample ID, and the amounts of components used in these laboratory-scale tests are shown in Table 6.17.

Table 6.17. PEP Parallel Test A Matrix

\begin{tabular}{ccccc}
\hline & & PEP Sample & 19M NaOH & DI Water \\
PEP Sample ID & Lab Test ID & $(\mathrm{g})$ & $(\mathrm{g})$ & $(\mathrm{g})$ \\
\hline A_01AIM_002_XX_1523_CUF_4 & A_LAB_CL_1 & 684.01 & --- & 116.04 \\
A_T22GM_002_XX_1508_CUF_4 & A_LAB_CL_2 & 510.01 & 166.01 & 124.00 \\
\hline
\end{tabular}

These tests were performed by placing the required amount of PEP simulant, $\mathrm{NaOH}$, and DI water in the test vessel, attaching the lid, and heating to $57^{\circ} \mathrm{C}$ while stirring. At $57^{\circ} \mathrm{C}$, the vessel contents were heated at a linear rate to $98 \pm 2{ }^{\circ} \mathrm{C}$ over a 4-hour and 10-minute time span. When the vessel reached $88 \pm 2{ }^{\circ} \mathrm{C}$, a sample was taken as the " $-0.95 \mathrm{hr}$ " target sample. At $98 \pm 2^{\circ} \mathrm{C}$, a sample was taken (time 0 ), and the test was held for 24 hours at this temperature. Samples were taken at 1, 2, 4, 8, 10, 12, 14, 16, 20, 22 , and 24 hours and immediately filtered through a $0.45-\mu \mathrm{m}$ syringe filter. These samples were then analyzed by ICP-AES for Al, Cr, and Na concentration at SWRI, and the results are shown in Table 6.18 through Table 6.20. Slurry samples were also taken at the beginning and end of the test. Some slurry samples were washed three times with $0.01 \mathrm{M} \mathrm{NaOH}$ and others were not washed. These samples were analyzed by ICP-AES for major cations at SWRI and these results are shown in Table 6.21 and Table 6.22. The samples were also analyzed by IC for anion concentrations, which are shown in Table 6.23 and Table 6.24 for the supernate and Table 6.25 for the slurries. The $\mathrm{OH}^{-}$concentration was also measured on the supernates and the slurries and is shown in Table 6.26 and Table 6.27 . The wt\% solids for the two tests were also measured and are shown in Table 6.28 and Table 6.29. 
Table 6.18. Supernate Al Analysis from PEP Parallel Test A Caustic Leach

\begin{tabular}{cccc}
\hline $\begin{array}{c}\text { Time } \\
(\mathrm{hrs})\end{array}$ & $\begin{array}{c}\text { Temperature } \\
\left({ }^{\circ} \mathrm{C}\right)\end{array}$ & $\begin{array}{c}\text { A_LAB_CL_1 } \\
(\mathrm{mg} \mathrm{A1} / \mathrm{kg} \text { soln })\end{array}$ & $\begin{array}{c}\text { A_LAB_CL_2 } \\
(\mathrm{mg} \mathrm{A1} / \mathrm{kg} \text { soln })\end{array}$ \\
\hline Initial & 23.2 & 4,610 & 2,000 \\
-0.95 & 88.0 & 6,360 & 6,050 \\
0 & 98.2 & 6,550 & 6,140 \\
1 & 98.0 & 6,730 & 6,270 \\
2 & 98.0 & 6,580 & 6,430 \\
4 & 98.1 & 7,220 & 5,810 \\
8 & 98.1 & 7,790 & 7,210 \\
10 & 98.0 & 7,570 & 7,460 \\
12 & 97.4 & 8,060 & 8,320 \\
14 & 97.8 & 8,340 & 9,200 \\
16 & 97.9 & 8,330 & 9,190 \\
18 & 98.0 & 8,610 & 9,620 \\
20 & 98.0 & 8,800 & 9,960 \\
22 & 97.9 & 8,970 & 10,300 \\
24 & 98.1 & 9,600 & 10,600 \\
Final & 57.8 & 9,320 & 10,500 \\
\hline
\end{tabular}

Table 6.19. Supernate Cr Analysis from PEP Parallel Test A Caustic Leach

\begin{tabular}{cccc}
\hline $\begin{array}{c}\text { Time } \\
(\mathrm{hrs})\end{array}$ & $\begin{array}{c}\text { Temperature } \\
\left({ }^{\circ} \mathrm{C}\right)\end{array}$ & $\begin{array}{c}\text { A_LAB_CL_1 } \\
(\mathrm{mg} \text { Cr/kg soln })\end{array}$ & $\begin{array}{c}\text { A_LAB_CL_2 } \\
(\mathrm{mg} \text { Cr/kg soln })\end{array}$ \\
\hline Initial & 23.2 & 1.05 & 0.97 \\
-0.95 & 88.0 & 1.17 & 1.32 \\
0 & 98.2 & 1.31 & 1.65 \\
1 & 98.0 & 1.36 & 2.26 \\
2 & 98.0 & 1.41 & 2.09 \\
4 & 98.1 & 1.81 & 2.10 \\
8 & 98.1 & 1.45 & 2.87 \\
10 & 98.0 & 1.43 & 3.06 \\
12 & 97.4 & 130 & 3.47 \\
14 & 97.8 & 1.64 & 3.57 \\
\hline 16 & 97.9 & 1.58 & 3.55 \\
18 & 98.0 & 1.50 & 3.73 \\
\hline 20 & 98.0 & 1.51 & 3.80 \\
\hline 22 & 97.9 & 1.50 & 3.71 \\
\hline 24 & 98.1 & 1.53 & 4.06 \\
\hline Final & 57.8 & 1.52 & 3.80 \\
\hline
\end{tabular}


Table 6.20. Supernate Na Analysis from PEP Parallel Test A Caustic Leach

\begin{tabular}{cccc}
\hline $\begin{array}{c}\text { Time } \\
(\mathrm{hrs})\end{array}$ & $\begin{array}{c}\text { Temperature } \\
\left({ }^{\circ} \mathrm{C}\right)\end{array}$ & $\begin{array}{c}\text { A_LAB_CL_1 } \\
(\mathrm{mg} \mathrm{Na} / \mathrm{kg} \text { soln })\end{array}$ & $\begin{array}{c}\text { A_LAB_CL_2 } \\
(\mathrm{mg} \mathrm{Na} / \mathrm{kg} \text { soln })\end{array}$ \\
\hline Initial & 23.2 & 118,000 & 109,000 \\
-0.95 & 88.0 & 122,000 & 120,000 \\
0 & 98.2 & 123,000 & 121,000 \\
1 & 98.0 & 126,000 & 121,000 \\
2 & 98.0 & 122,000 & 121,000 \\
4 & 98.1 & 127,000 & 106,000 \\
8 & 98.1 & 131,000 & 125,000 \\
10 & 98.0 & 124,000 & 125,000 \\
12 & 97.4 & 127,000 & 134,000 \\
14 & 97.8 & 129,000 & 145,000 \\
16 & 97.9 & 127,000 & 141,000 \\
18 & 98.0 & 127,000 & 145,000 \\
20 & 98.0 & 128,000 & 147,000 \\
22 & 97.9 & 127,000 & 147,000 \\
24 & 98.1 & 134,000 & 147,000 \\
Final & 57.8 & 130,000 & 148,000 \\
\hline
\end{tabular}


Table 6.21. Slurry Analysis from PEP Parallel Test A Caustic Leach Test 1

\begin{tabular}{|c|c|c|c|c|c|c|c|}
\hline & $\begin{array}{l}\text { A_LAB_- } \\
\text { CL_1_IS2_SL } \\
\text { (mg/kg slurry) }\end{array}$ & $\begin{array}{l}\text { A_LAB_- } \\
\text { CL_1_IS3_SL } \\
\text { (mg/kg slurry) }\end{array}$ & $\begin{array}{c}\text { A_LAB_- } \\
\text { CL_1_ISW_SL } \\
\text { (mg/kg slurry) }\end{array}$ & $\begin{array}{l}\text { A_LAB_- } \\
\text { CL_1_FS1_SL } \\
\text { (mg/kg slurry) }\end{array}$ & $\begin{array}{l}\text { A_LAB_- } \\
\text { CL_1_FS2_SL } \\
\text { (mg/kg slurry) }\end{array}$ & $\begin{array}{l}\text { A_LAB_- } \\
\text { CL_1_FS3_SL } \\
\text { (mg/kg slurry) }\end{array}$ & $\begin{array}{l}\text { A_LAB_- } \\
\text { CL_1_FS4_SL } \\
\text { (mg/kg slurry) }\end{array}$ \\
\hline & Initial Slurry-2 & Initial Slurry-3 & Initial Washed Slurry & Final Washed Slurry-1 & Final Washed Slurry-2 & Final Washed Slurry-3 & Final Slurry-4 \\
\hline $\mathrm{Al}$ & 9,490 & 9,560 & 17,100 & 10,100 & 10,000 & 9,790 & 11,600 \\
\hline $\mathrm{Ca}$ & 92.1 & 91.0 & 280 & 333 & 329 & 330 & 97.4 \\
\hline $\mathrm{Cr}$ & 1.64 & 1.67 & 1.89 & 1.09 & 1.04 & 1.06 & 1.88 \\
\hline $\mathrm{Fe}$ & 2,910 & 2,880 & 9,070 & 10,800 & 10,500 & 10,500 & 3,030 \\
\hline $\mathrm{Mg}$ & 62.4 & 61.8 & 190 & 229 & 227 & 227 & 64.0 \\
\hline $\mathrm{Mn}$ & 623 & 624 & 1,980 & 2,350 & 2,280 & 2,260 & 660 \\
\hline $\mathrm{Ni}$ & 92.6 & 91.8 & 289 & 353 & 347 & 345 & 96.3 \\
\hline $\mathrm{Na}$ & 117,000 & 115,000 & 466 & 664 & 812 & 631 & 125,000 \\
\hline $\mathrm{P}$ & 974 & 1,040 & 57.1 & 61.9 & 60.2 & 57.7 & 1,090 \\
\hline $\mathrm{Sr}$ & 26.2 & 26.2 & 80.2 & 94.0 & 92.0 & 91.7 & 27.9 \\
\hline $\mathrm{Zr}$ & 52.3 & 56.3 & 245 & 251 & 243 & 264 & 74.8 \\
\hline
\end{tabular}

vi

Table 6.22. Slurry Analysis from PEP Parallel Test A Caustic Leach Test 2

\begin{tabular}{|c|c|c|c|c|c|c|c|}
\hline & $\begin{array}{l}\text { A_LAB_ } \\
\text { CL_2_IS2_SL } \\
\text { (mg/kg slurry) }\end{array}$ & $\begin{array}{l}\text { A_LAB_ } \\
\text { CL_2_IS3_SL } \\
\text { (mg/kg slurry) }\end{array}$ & $\begin{array}{l}\text { A_LAB_- } \\
\text { CL_2_ISW_SL } \\
\text { (mg/kg slurry) }\end{array}$ & $\begin{array}{l}\text { A_LAB_ } \\
\text { CL_2_FS1_SL } \\
(\mathrm{mg} / \mathrm{kg} \text { slurry) }\end{array}$ & $\begin{array}{l}\text { A_LAB_ } \\
\text { CL_2_FS2_SL } \\
(\mathrm{mg} / \mathrm{kg} \text { slurry) }\end{array}$ & $\begin{array}{c}\text { A_LAB_- } \\
\text { CL_2_FS3_SL } \\
(\mathrm{mg} / \mathrm{kg} \text { slurry })\end{array}$ & $\begin{array}{l}\text { A_LAB_- } \\
\text { CL_2_FS4_SL } \\
\text { (mg/kg slurry) }\end{array}$ \\
\hline & Initial Slurry-2 & Initial Slurry-3 & Initial Washed Slurry & Final Washed Slurry-1 & Final Washed Slurry-2 & Final Washed Slurry-3 & Final Slurry-4 \\
\hline $\mathrm{Al}$ & 9,340 & 9,230 & 19,700 & 9,180 & 9,100 & 9,500 & 13,000 \\
\hline $\mathrm{Ca}$ & 87.3 & 85.1 & 256 & 353 & 355 & 366 & 105 \\
\hline $\mathrm{Cr}$ & 1.63 & 1.54 & 1.71 & 1.15 & 1.20 & 1.25 & 3.77 \\
\hline $\mathrm{Fe}$ & 2,870 & 2,800 & 8,670 & 11,600 & 11,800 & 12,400 & 3,410 \\
\hline $\mathrm{Mg}$ & 63.8 & 59.3 & 184 & 251 & 255 & 262 & 73.6 \\
\hline $\mathrm{Mn}$ & 609 & 611 & 1,870 & 2,460 & 2,500 & 2,640 & 733 \\
\hline $\mathrm{Ni}$ & 91.0 & 87.2 & 277 & 373 & 372 & 391 & 107 \\
\hline $\mathrm{Na}$ & 115,000 & 113,000 & 626 & 855 & 873 & 890 & 139,000 \\
\hline $\mathrm{P}$ & 724 & 680 & 62.0 & 50.2 & 47.5 & 54.9 & 1,230 \\
\hline $\mathrm{Sr}$ & 25.9 & 25.8 & 76.3 & 102 & 102 & 107 & 31.1 \\
\hline $\mathrm{Zr}$ & 56.0 & 54 & 237 & 273 & 271 & 282 & 88.8 \\
\hline
\end{tabular}


Table 6.23. Anion Analysis of A_LAB_CL_1 Supernate

\begin{tabular}{cccccccc}
\hline Time $(\mathrm{hrs})$ & $\begin{array}{c}\text { Temperature } \\
\left({ }^{\circ} \mathrm{C}\right)\end{array}$ & $\begin{array}{c}\text { Chloride } \\
(\mathrm{mg} / \mathrm{kg} \text { soln })\end{array}$ & $\begin{array}{c}\text { Nitrate } \\
(\mathrm{mg} / \mathrm{kg} \text { soln })\end{array}$ & $\begin{array}{c}\text { Nitrite } \\
(\mathrm{mg} / \mathrm{kg} \text { soln })\end{array}$ & $\begin{array}{c}\text { Oxalate } \\
(\mathrm{mg} / \mathrm{kg} \text { soln })\end{array}$ & $\begin{array}{c}\text { Phosphate } \\
(\mathrm{mg} / \mathrm{kg} \text { soln })\end{array}$ & $\begin{array}{c}\text { Sulfate } \\
(\mathrm{mg} / \mathrm{kg} \text { soln })\end{array}$ \\
\hline Initial & 23.2 & 736 & 55,400 & 12,300 & 183 & 1,940 & 9,490 \\
-0.95 & 88.0 & 763 & 57,100 & 12,600 & 969 & 3,800 & 9,810 \\
0 & 98.2 & 736 & 54,900 & 12,400 & 1,030 & 3,740 & 9,540 \\
1 & 98.0 & 775 & 58,000 & 12,900 & 1,200 & 3,860 & 9,960 \\
2 & 98.0 & 760 & 56,700 & 12,600 & 1,220 & 3,770 & 9,700 \\
4 & 98.1 & 768 & 57,100 & 12,700 & 1,130 & 3,800 & 9,810 \\
8 & 98.1 & 794 & 59,300 & 13,200 & 1,080 & 3,770 & 10,100 \\
10 & 98.0 & 778 & 57,600 & 12,800 & 1,070 & 3,770 & 9,850 \\
12 & 97.4 & 800 & 58,900 & 13,000 & 1,050 & 3,980 & 9,990 \\
14 & 97.8 & 793 & 59,300 & 13,100 & 1,090 & 3,430 & 10,100 \\
16 & 97.9 & 793 & 58,900 & 13,100 & 932 & 3,620 & 9,960 \\
18 & 98.0 & 817 & 60,700 & 13,400 & 671 & 3,490 & 10,000 \\
20 & 98.0 & 805 & 59,800 & 13,300 & 882 & 3,890 & 9,920 \\
22 & 97.9 & 797 & 59,300 & 13,200 & 855 & 3,890 & 9,930 \\
24 & 98.1 & 854 & 63,300 & 14,100 & 946 & 4,510 & 10,500 \\
Final & 57.8 & 812 & 61,100 & 13,600 & 377 & 3,920 & 10,300 \\
\hline
\end{tabular}

Table 6.24. Anion Analysis of A_LAB_CL_2 Supernate

\begin{tabular}{cccccccc}
\hline Time $(\mathrm{hrs})$ & $\begin{array}{c}\text { Temperature } \\
\left({ }^{\circ} \mathrm{C}\right)\end{array}$ & $\begin{array}{c}\text { Chloride } \\
(\mathrm{mg} / \mathrm{kg} \text { soln })\end{array}$ & $\begin{array}{c}\text { Nitrate } \\
(\mathrm{mg} / \mathrm{kg} \text { soln })\end{array}$ & $\begin{array}{c}\text { Nitrite } \\
(\mathrm{mg} / \mathrm{kg} \text { soln })\end{array}$ & $\begin{array}{c}\text { Oxalate } \\
(\mathrm{mg} / \mathrm{kg} \text { soln })\end{array}$ & $\begin{array}{c}\text { Phosphate } \\
(\mathrm{mg} / \mathrm{kg} \text { soln })\end{array}$ & $\begin{array}{c}\text { Sulfate } \\
(\mathrm{mg} / \mathrm{kg} \text { soln })\end{array}$ \\
\hline Initial & 23.2 & 741 & 51,800 & 12,000 & 323 & 3,710 & 9,330 \\
-0.95 & 88.0 & 758 & 53,100 & 12,500 & 1,000 & 3,770 & 9,640 \\
0 & 98.2 & 789 & 57,600 & 12,700 & 1,310 & 3,830 & 9,910 \\
1 & 98.0 & 756 & 56,200 & 12,500 & 1,350 & 3,740 & 9,650 \\
2 & 98.0 & 778 & 57,100 & 12,700 & 1,250 & 3,770 & 9,790 \\
4 & 98.1 & 775 & 56,700 & 12,500 & 1,210 & 3,740 & 9,770 \\
8 & 98.1 & 778 & 54,000 & 12,600 & 1,060 & 3,590 & 9,650 \\
10 & 98.0 & 779 & 54,500 & 12,500 & 1,200 & 3,800 & 9,640 \\
12 & 97.4 & 863 & 60,200 & 13,600 & 1,340 & 3,590 & 10,400 \\
14 & 97.8 & 932 & 64,700 & 14,900 & 886 & 4,510 & 11,400 \\
16 & 97.9 & 922 & 64,200 & 14,700 & 720 & 4,170 & 11,100 \\
18 & 98.0 & 976 & 67,800 & 15,400 & 640 & 4,200 & 11,600 \\
20 & 98.0 & 960 & 66,400 & 15,200 & 711 & 4,290 & 11,400 \\
22 & 97.9 & 968 & 67,300 & 15,100 & 728 & 4,410 & 11,300 \\
24 & 98.1 & 975 & 67,800 & 15,300 & 718 & 4,510 & 11,300 \\
Final & 57.8 & 965 & 66,900 & 15,300 & 293 & 4,260 & 11,500 \\
\hline
\end{tabular}


Table 6.25. Anion Analysis of Slurry Samples

\begin{tabular}{|c|c|c|c|c|c|c|c|}
\hline Sample ID & $\begin{array}{c}\text { Sample } \\
\text { Description }\end{array}$ & $\begin{array}{c}\text { Chloride } \\
\text { (mg/kg } \\
\text { slurry) }\end{array}$ & $\begin{array}{l}\text { Nitrate } \\
\text { (mg/kg } \\
\text { slurry) }\end{array}$ & $\begin{array}{l}\text { Nitrite } \\
(\mathrm{mg} / \mathrm{kg} \\
\text { slurry) }\end{array}$ & $\begin{array}{l}\text { Oxalate } \\
\text { (mg/kg } \\
\text { slurry) }\end{array}$ & $\begin{array}{c}\text { Phosphate } \\
\text { (mg/kg } \\
\text { slurry) }\end{array}$ & $\begin{array}{l}\text { Sulfate } \\
(\mathrm{mg} / \mathrm{kg} \\
\text { slurry) }\end{array}$ \\
\hline A_LAB_CL_1_ISW_SL & $\begin{array}{c}\text { Test } 1 \text { Initial } \\
\text { Washed } \\
\text { Slurry }\end{array}$ & $<18.7$ & $<83$ & $<61$ & $<18.7$ & $<57$ & $<18.7$ \\
\hline A_LAB_CL_1_IS2_SL & $\begin{array}{c}\text { Test } 1 \text { Initial } \\
\text { Slurry }-1\end{array}$ & 631 & 45,600 & 10,700 & 2,620 & 3,340 & 8,310 \\
\hline A_LAB_CL_1_IS3_SL & $\begin{array}{c}\text { Test } 1 \text { Initial } \\
\text { Slurry -2 }\end{array}$ & 636 & 46,100 & 10,700 & 2,590 & 3,190 & 8,370 \\
\hline A_LAB_CL_1_FS1_SL & $\begin{array}{c}\text { Test } 1 \text { Final } \\
\text { Washed } \\
\text { Slurry -1 }\end{array}$ & $<19.3$ & 86 & $<63$ & $<19.3$ & 129 & $<19.3$ \\
\hline A_LAB_CL_1_FS2_SL & $\begin{array}{c}\text { Test } 1 \text { Final } \\
\text { Washed } \\
\text { Slurry -2 }\end{array}$ & $<18.8$ & 85 & $<62$ & $<18.8$ & 138 & $<18.8$ \\
\hline A_LAB_CL_1_FS3_SL & $\begin{array}{c}\text { Test } 1 \text { Final } \\
\text { Washed } \\
\text { Slurry -3 }\end{array}$ & $<19.5$ & 89 & $<64$ & $<19.5$ & 166 & $<19.5$ \\
\hline A_LAB_CL_1_FS4_SL & $\begin{array}{c}\text { Test } 1 \text { Final } \\
\text { Slurry }-3\end{array}$ & 695 & 50,000 & 11,600 & 2,770 & 2,620 & 9,100 \\
\hline A_LAB_CL_2_ISW_SL & $\begin{array}{c}\text { Test } 2 \text { Initial } \\
\text { Washed } \\
\text { Slurry }\end{array}$ & $<18.8$ & $<83$ & $<62$ & $<18.8$ & $<58$ & $<18.8$ \\
\hline A_LAB_CL_2_IS2_SL & $\begin{array}{l}\text { Test } 2 \text { Initial } \\
\text { Slurry }-1\end{array}$ & 659 & 46,500 & 10,800 & 2,040 & 1,960 & 8,370 \\
\hline A_LAB_CL_2_IS3_SL & $\begin{array}{c}\text { Test } 2 \text { Initial } \\
\text { Slurry }-2\end{array}$ & 655 & 46,900 & 11,100 & 2,180 & 1,970 & 8,450 \\
\hline A_LAB_CL_2_FS1_SL & $\begin{array}{c}\text { Test } 2 \text { Final } \\
\text { Washed } \\
\text { Slurry -1 }\end{array}$ & $<19.7$ & 90 & $<65$ & $<19.7$ & 166 & $<19.7$ \\
\hline A_LAB_CL_2_FS2_SL & $\begin{array}{c}\text { Test } 2 \text { Final } \\
\text { Washed } \\
\text { Slurry -2 }\end{array}$ & $<19.1$ & 87 & $<63$ & $<19.1$ & 142 & $<19.1$ \\
\hline A_LAB_CL_2_FS3_SL & $\begin{array}{c}\text { Test } 2 \text { Final } \\
\text { Washed } \\
\text { Slurry }-3\end{array}$ & $<18.9$ & 85 & $<62$ & $<18.9$ & 161 & $<18.9$ \\
\hline A_LAB_CL_2_FS4_SL & $\begin{array}{l}\text { Test } 2 \text { Final } \\
\text { Slurry -3 }\end{array}$ & 808 & 57,100 & 13,300 & 2,590 & 3,800 & 10,100 \\
\hline
\end{tabular}


Table 6.26. PEP Parallel Test A Free OH Supernate Concentration

\begin{tabular}{lclc}
\hline \multicolumn{1}{c}{ Sample ID } & $\begin{array}{c}\text { OH Conc. } \\
(\mathrm{mol} / \mathrm{L})\end{array}$ & \multicolumn{1}{c}{ Sample ID } & $\begin{array}{c}\text { OH Conc. } \\
(\mathrm{mol} / \mathrm{L})\end{array}$ \\
\hline A_LAB_CL_1_IN_SUP & 4.06 & A_LAB_CL_2_IN_SUP & 3.87 \\
A_LAB_CL_1_010C & 4.05 & A_LAB_CL_2_010C & 3.84 \\
\hline A_LAB_CL_1_000 & 4.08 & A_LAB_CL_2_000 & 3.70 \\
A_LAB_CL_1_001 & 4.00 & A_LAB_CL_2_001 & 3.76 \\
A_LAB_CL_1_002 & 3.97 & A_LAB_CL_2_002 & 3.62 \\
A_LAB_CL_1_004 & 3.87 & A_LAB_CL_2_004 & 3.61 \\
A_LAB_CL_1_008 & 3.95 & A_LAB_CL_2_008 & 3.76 \\
A_LAB_CL_1_010 & 3.94 & A_LAB_CL_2_010 & 4.00 \\
A_LAB_CL_1_012 & 4.16 & A_LAB_CL_2_012 & 4.49 \\
A_LAB_CL_1_014 & 4.01 & A_LAB_CL_2_014 & 4.83 \\
A_LAB_CL_1_016 & 4.00 & A_LAB_CL_2_016 & 4.81 \\
A_LAB_CL_1_018 & 4.06 & A_LAB_CL_2_018 & 4.90 \\
A_LAB_CL_1_020 & 4.34 & A_LAB_CL_2_020 & 4.81 \\
A_LAB_CL_1_022 & 4.13 & A_LAB_CL_2_022 & 4.88 \\
A_LAB_CL_1_024 & 3.96 & A_LAB_CL_2_024 & 4.75 \\
A_LAB_CL_1_F & 4.01 & A_LAB_CL_2_F & 4.73 \\
\hline
\end{tabular}

Table 6.27. PEP Parallel Test A Slurry Free OH Concentration

\begin{tabular}{|c|c|c|c|c|c|}
\hline Sample ID & $\begin{array}{c}\text { Sample } \\
\text { Description }\end{array}$ & $\begin{array}{c}\mathrm{OH} \\
\text { Conc. } \\
\text { (meq } / \mathrm{g} \text { ) }\end{array}$ & Sample ID & $\begin{array}{c}\text { Sample } \\
\text { Description }\end{array}$ & $\begin{array}{c}\mathrm{OH} \\
\text { Conc. } \\
\text { (meq/g) }\end{array}$ \\
\hline A_LAB_CL_1_IS_SL & $\begin{array}{l}\text { Test } 1 \text { Initial } \\
\text { Slurry }\end{array}$ & 0.0320 & A_LAB_CL_3_IS_SL & $\begin{array}{c}\text { Test } 3 \text { Initial } \\
\text { Slurry }\end{array}$ & 0.0283 \\
\hline A_LAB_CL_1_FS1_SL & $\begin{array}{c}\text { Test } 1 \text { Final } \\
\text { Washed Slurry-1 }\end{array}$ & 0.0417 & A_LAB_CL_3_FS1_SL & $\begin{array}{c}\text { Test } 3 \text { Final } \\
\text { Washed Slurry-1 }\end{array}$ & 0.0474 \\
\hline A_LAB_CL_1_FS2_SL & $\begin{array}{c}\text { Test } 1 \text { Final } \\
\text { Washed Slurry-2 }\end{array}$ & 0.0483 & A_LAB_CL_3_FS2_SL & $\begin{array}{c}\text { Test } 3 \text { Final } \\
\text { Washed Slurry-2 }\end{array}$ & 0.0424 \\
\hline A_LAB_CL_1_FS3_SL & $\begin{array}{c}\text { Test } 1 \text { Final } \\
\text { Washed Slurry-3 }\end{array}$ & 0.0425 & A_LAB_CL_3_FS3_SL & $\begin{array}{c}\text { Test } 3 \text { Final } \\
\text { Washed Slurry-3 }\end{array}$ & 0.0399 \\
\hline A_LAB_CL_2_IS_SL & $\begin{array}{l}\text { Test } 2 \text { Initial } \\
\text { Slurry }\end{array}$ & 0.0850 & A_LAB_CL_4_IS_SL & $\begin{array}{c}\text { Test } 4 \text { Initial } \\
\text { Slurry }\end{array}$ & 0.0661 \\
\hline A_LAB_CL_2_FS1_SL & $\begin{array}{c}\text { Test } 2 \text { Final } \\
\text { Washed Slurry-1 }\end{array}$ & 0.105 & A_LAB_CL_4_FS1_SL & $\begin{array}{c}\text { Test } 4 \text { Final } \\
\text { Washed Slurry-1 }\end{array}$ & 0.111 \\
\hline A_LAB_CL_2_FS2_SL & $\begin{array}{c}\text { Test } 2 \text { Final } \\
\text { Washed Slurry-2 }\end{array}$ & 0.111 & A_LAB_CL_4_FS2 2 SL & $\begin{array}{c}\text { Test } 4 \text { Final } \\
\text { Washed Slurry-2 }\end{array}$ & 0.111 \\
\hline A_LAB_CL_2_FS3_SL & $\begin{array}{c}\text { Test } 2 \text { Final } \\
\text { Washed Slurry-3 }\end{array}$ & 0.118 & A_LAB_CL_4_FS3_SL & $\begin{array}{c}\text { Test } 4 \text { Final } \\
\text { Washed Slurry-3 }\end{array}$ & 0.110 \\
\hline
\end{tabular}


Table 6.28. PEP Parallel Test A Physical Properties for Test 1

\begin{tabular}{lccc}
\hline \multicolumn{1}{c}{ Property } & A_LAB_CL_1_ISW_SL & A_LAB_CL_1_IS2_SL & A_LAB_CL_1_IS3_SL \\
\hline Sample Description & Initial Washed Slurry & Initial Slurry-2 & Initial Slurry-3 \\
Bulk Density $(\mathrm{g} / \mathrm{mL})$ & 1.044 & 1.325 & 1.304 \\
Density of Cent. Solids $(\mathrm{g} / \mathrm{mL})$ & 1.354 & 1.756 & 1.812 \\
Vol\% of Cent. Solids & 16.0 & 10.2 & 10.0 \\
Wt\% Cent. Solids & 20.7 & 13.5 & 13.9 \\
Supernate Density $(\mathrm{g} / \mathrm{mL})$ & 0.983 & 1.272 & 1.243 \\
Wt\% Total Solids & 7.15 & 31.8 & 31.9 \\
Wt\% Oven Dried Solids & 34.2 & 41.3 & 41.3 \\
Wt\% UDS & 7.10 & 2.12 & 2.17 \\
Wt\% Dissolved Solids & 0.056 & 30.4 & 30.4 \\
Wt\% Supernate Liquid & 79.1 & 86.2 & 85.8 \\
\hline
\end{tabular}

Table 6.28 (cont)

\begin{tabular}{|c|c|c|c|c|}
\hline Property & $\begin{array}{c}\text { A_LAB_CL_1 } \\
\text { FS1_SL }\end{array}$ & $\begin{array}{c}\text { A_LAB_CL_1 } \\
\text { FS2_SL }\end{array}$ & $\begin{array}{c}\text { A_LAB_CL_1 } \\
\text { FS3_SL }\end{array}$ & $\begin{array}{c}\text { A_LAB_CL_1 } \\
\text { FS4_SL }\end{array}$ \\
\hline Sample Description & Final Washed Slurry-1 & $\begin{array}{c}\text { Final Washed } \\
\text { Slurry-2 }\end{array}$ & $\begin{array}{c}\text { Final Washed } \\
\text { Slurry-3 }\end{array}$ & Final Slurry \\
\hline Bulk Density $(\mathrm{g} / \mathrm{mL})$ & 1.017 & 1.016 & 1.017 & 1.361 \\
\hline Density of Cent. Solids $(\mathrm{g} / \mathrm{mL})$ & 1.451 & 1.372 & 1.421 & 1.853 \\
\hline Vol\% of Cent. Solids & 12.0 & 11.8 & 11.8 & 10.4 \\
\hline Wt\% Cent. Solids & 17.1 & 15.9 & 16.4 & 14.2 \\
\hline Supernate Density $(\mathrm{g} / \mathrm{mL})$ & 0.955 & 0.960 & 0.954 & 1.300 \\
\hline Wt $\%$ Total Solids & 5.23 & 5.12 & 5.13 & 33.8 \\
\hline Wt $\%$ Oven Dried Solids & 30.3 & 32.0 & 30.9 & 40.3 \\
\hline $\mathrm{Wt} \%$ UDS & 5.18 & 5.08 & 5.06 & 1.59 \\
\hline Wt\% Dissolved Solids & 0.057 & 0.039 & 0.072 & 32.8 \\
\hline Wt $\%$ Supernate Liquid & 82.6 & 83.4 & 82.8 & 85.6 \\
\hline
\end{tabular}

Table 6.29. PEP Parallel Test A Physical Properties for Test 2

\begin{tabular}{lccc}
\hline \multicolumn{1}{c}{ Property } & A_LAB_CL_2_ISW_SL & A_LAB_CL_2_IS2_SL & A_LAB_CL_2_IS3_SL \\
\hline Sample Description & Initial Washed Slurry & Initial Slurry-2 & Initial Slurry-3 \\
\hline Bulk Density $(\mathrm{g} / \mathrm{mL})$ & 1.033 & 1.287 & 1.298 \\
Density of Cent. Solids $(\mathrm{g} / \mathrm{mL})$ & 1.439 & 1.553 & 1.651 \\
Vol\% of Cent. Solids & 15.7 & 10.0 & 10.0 \\
Wt\% Cent. Solids & 21.9 & 12.1 & 12.7 \\
Supernate Density $(\mathrm{g} / \mathrm{mL})$ & 0.953 & 1.254 & 1.258 \\
Wt $\%$ Total Solids & 7.70 & 31.3 & 31.1 \\
Wt $\%$ Oven Dried Solids & 35.2 & 40.0 & 41.1 \\
Wt\% UDS & 7.70 & 1.71 & 2.07 \\
Wt $\%$ Dissolved Solids & 0.005 & 30.1 & 29.6 \\
Wt $\%$ Supernate Liquid & 77.8 & 87.7 & 87.2 \\
\hline
\end{tabular}


Table 6.29 (cont)

\begin{tabular}{|c|c|c|c|c|}
\hline Property & $\begin{array}{c}\text { A_LAB_CL_2 } \\
\text { FS1_SL }\end{array}$ & $\begin{array}{c}\text { A_LAB_CL_2 } \\
\text { FS2_SL }\end{array}$ & $\begin{array}{c}\text { A_LAB_CL_2 } \\
\text { FS3_SL }\end{array}$ & $\begin{array}{c}\text { A_LAB_CL_2 } \\
\text { FS4_SL }\end{array}$ \\
\hline Sample Description & $\begin{array}{c}\text { Final Washed } \\
\text { Slurry-1 }\end{array}$ & $\begin{array}{c}\text { Final Washed } \\
\text { Slurry-2 }\end{array}$ & $\begin{array}{c}\text { Final Washed } \\
\text { Slurry-3 }\end{array}$ & Final Slurry \\
\hline Bulk Density $(\mathrm{g} / \mathrm{mL})$ & 1.014 & 1.042 & 1.023 & 1.382 \\
\hline Density of Cent. Solids $(\mathrm{g} / \mathrm{mL})$ & 1.465 & 1.541 & 1.655 & 2.313 \\
\hline Vol\% of Cent. Solids & 11.8 & 12.0 & 11.8 & 10.2 \\
\hline Wt $\%$ Cent. Solids & 17.0 & 17.7 & 19.0 & 17.1 \\
\hline Supernate Density $(\mathrm{g} / \mathrm{mL})$ & 0.950 & 0.971 & 0.935 & 1.274 \\
\hline Wt\% Total Solids & 5.25 & 5.36 & 5.75 & 38.2 \\
\hline Wt $\%$ Oven Dried Solids & 30.6 & 30.2 & 29.9 & 42.1 \\
\hline $\mathrm{Wt} \%$ UDS & 5.20 & 5.35 & 5.68 & 1.27 \\
\hline $\mathrm{Wt} \%$ Dissolved Solids & 0.049 & 0.00 & 0.076 & 37.5 \\
\hline $\mathrm{Wt} \%$ Supernate Liquid & 82.6 & 82.0 & 80.7 & 82.8 \\
\hline
\end{tabular}

\subsection{PEP Test B Caustic Leach}

The caustic leach process for PEP Test B was conducted under Test Instruction TI-WTP-PEP-066 (TI-066) in the PEP UFP-VSL-T02A tank and was completed as follows. Feed stored in HLP-VSL-T22 was transferred into vessel UFP-VSL-T01A. AFA was added directly to UFP-VSL-T01A in a quantity expected to produce a nominal concentration of $350 \mathrm{ppm}$ in the slurry. The slurry was then transferred from the UFP-VSL-T01A feed tank to UFP-VSL-T02A, and permeate was removed from UFP-VSL-T02A through the first ultrafilter bundle to increase the solids concentration of the slurry. As permeate was removed, the volume (and level) in UFP-VSL-T02A fell, triggering the transfer of small refill batches (i.e., $11 \mathrm{gal}$ ) of fresh simulant from UFP-VSL-T01A. The filtering and refill process continued, leaving a target quantity of slurry at about 20-wt\% UDS in the UFP-VSL-T02A vessel and filter loop. When the solids concentration process was complete, the permeate valves were closed on the filter system, caustic reagent was introduced upstream of the filter loop pumps, and more AFA was added to maintain the 350-ppm target concentration.

The caustic slurry in UFP-VSL-T02A was heated to about $71^{\circ} \mathrm{C}$ using the heat of dilution of the concentrated $\mathrm{NaOH}$ and mechanical heat from the filter loop recirculation pumps. The filter loop pumps were then turned off, and a portion of the concentrated slurry in the filter loop was flushed back into UFP-VSL-T02A before the loop was closed off from the vessel. Because the total loop volume (82 gal excluding dead volume) was significantly greater than the flush volume ( $\sim 20$ gal), none of the flush solution $(0.01 \mathrm{M} \mathrm{NaOH})$ is expected to have entered the vessel. After the flush, a sample of slurry was taken from the middle-low region of vessel UFP-VSL-T02A using the CD sampler for use as feed in the laboratory-scale caustic leach tests (B_LAB_CL_1 and B_LAB_CL_2 as duplicates) on March 15, 2009 at $17: 15$. The samples were rapidly cooled in a cold water bath to ambient temperature ${ }^{(a)}$ to minimize any further leaching reaction.

(a) According to an interview with the lead sample handler, for one of the Test B laboratory-scale 1-L samples, rapid cooling proceeded only for about 1 hour before it had to be moved from the cold-water bath to make room in the bath for analytical PEP samples. Cooling of the laboratory-scale 1-L sample was continued by running it under cold water in the sink. 
The laboratory-scale feed was stored at laboratory ambient temperature until it was used. The delay between the time when the feed was acquired from PEP and the time laboratory-scale testing started was about 4 days for Test B (from March 15, 2009 to March 19, 2009).

The PEP sample ID, laboratory sample ID, and the amount of water used in the parallel laboratory-scale tests are shown in Table 6.30 .

Table 6.30. PEP Parallel Test B Matrix

\begin{tabular}{cccc}
\hline PEP Sample ID & $\begin{array}{c}\text { Laboratory } \\
\text { Test ID }\end{array}$ & $\begin{array}{c}\text { PEP Sample } \\
(\mathrm{g})\end{array}$ & $\begin{array}{c}\text { DI Water } \\
(\mathrm{g})\end{array}$ \\
\hline B_02AML_016_XX_4469_CUF_4 & B_LAB_CL_1 & 669.02 & 131.00 \\
B_02AML_016_XX_4469_CUF_4 & B_LAB_CL_2 & 669.01 & 131.02 \\
\hline
\end{tabular}

These tests were performed by placing the required amount of PEP simulant and DI water in the test vessel, attaching the lid, and heating to $71^{\circ} \mathrm{C}$ while stirring. At $71{ }^{\circ} \mathrm{C}$, the vessel contents were heated at a linear rate to $98 \pm 2{ }^{\circ} \mathrm{C}$ over a 2.6-hour time span. When the vessel reached $88 \pm 2{ }^{\circ} \mathrm{C}$, a sample was taken as the "- $0.95 \mathrm{hr}$ " sample. At $98 \pm 2{ }^{\circ} \mathrm{C}$, the time zero $(0 \mathrm{hr})$ sample was taken, and the test was held for 24 hours at this temperature. Samples were taken at 1, 2, 4, 8, 10, 12, 14, 16, 20, 22, and 24 hours and immediately filtered through a $0.45-\mu \mathrm{m}$ syringe filter. These samples were then analyzed by ICP-AES for $\mathrm{Al}, \mathrm{Cr}$, and Na concentration at SWRI, and the results are shown in Table 6.31 through Table 6.33. Slurry samples were also taken at the beginning and end of the test. Some slurry samples were washed three times with $0.01 \mathrm{M} \mathrm{NaOH}$ and others were not washed. These samples were analyzed by ICP-AES for major cations at SWRI and these results are shown in Table 6.34 through Table 6.35. The samples were also analyzed by IC for anion concentrations, which are shown in Table 6.36 and Table 6.37 for the supernate and Table 6.38 for the slurries. The $\mathrm{OH}^{-}$concentration, which was also measured on the supernates, is shown in Table 6.39. The wt \% solids of the slurry (both washed and unwashed) for each test were measured and are shown in Table 6.40 and Table 6.41.

Table 6.31. Supernate Al Analysis from PEP Parallel Test B Caustic Leach

\begin{tabular}{cccc}
\hline $\begin{array}{c}\text { Time } \\
(\mathrm{hrs})\end{array}$ & $\begin{array}{c}\text { Temperature } \\
\left({ }^{\circ} \mathrm{C}\right)\end{array}$ & $\begin{array}{c}\text { B-LAB_CL_1 } \\
(\mathrm{mg} \mathrm{Al} / \mathrm{kg} \mathrm{soln})\end{array}$ & $\begin{array}{c}\text { B-LAB_CL_2 } \\
(\mathrm{mg} \mathrm{A1} / \mathrm{kg} \text { soln })\end{array}$ \\
\hline Initial & 23.4 & 12,400 & 12,200 \\
-0.95 & 88.2 & 12,300 & 12,600 \\
0 & 98.2 & 13,000 & 12,200 \\
1 & 98.0 & 13,700 & 13,300 \\
2 & 98.0 & 13,600 & 13,400 \\
4 & 98.0 & 14,500 & 14,500 \\
8 & 98.0 & 15,800 & 16,300 \\
10 & 97.7 & 16,100 & 16,100 \\
12 & 98.0 & 16,900 & 16,800 \\
14 & 98.0 & 17,200 & 17,300 \\
16 & 98.2 & 17,700 & 18,200 \\
18 & 97.9 & 18,200 & 19,000 \\
21 & 98.0 & 18,900 & 19,300 \\
24 & 98.1 & 19,800 & 19,800 \\
\hline
\end{tabular}


Table 6.32. Supernate Cr Analysis from PEP Parallel Test B Caustic Leach

\begin{tabular}{cccc}
\hline $\begin{array}{c}\text { Time } \\
(\mathrm{hrs})\end{array}$ & $\begin{array}{c}\text { Temperature } \\
\left({ }^{\circ} \mathrm{C}\right)\end{array}$ & $\begin{array}{c}\text { B-LAB_CL_1 } \\
(\mathrm{mg} \mathrm{Cr} / \mathrm{kg} \text { soln })\end{array}$ & $\begin{array}{c}\text { B-LAB_CL_2 } \\
(\mathrm{mg} \mathrm{Cr} / \mathrm{kg} \text { soln })\end{array}$ \\
\hline Initial & 23.4 & 2.10 & 2.26 \\
-0.95 & 88.2 & 2.36 & 2.61 \\
0 & 98.2 & 3.03 & 2.80 \\
1 & 98.0 & 3.48 & 3.28 \\
2 & 98.0 & 3.41 & 3.39 \\
4 & 98.0 & 3.77 & 3.84 \\
8 & 98.0 & 3.92 & 3.86 \\
10 & 97.7 & 4.30 & 4.01 \\
12 & 98.0 & 4.21 & 4.05 \\
14 & 98.0 & 4.20 & 4.28 \\
16 & 98.2 & 4.40 & 4.36 \\
18 & 97.9 & 4.46 & 4.49 \\
21 & 98.0 & 4.58 & 4.44 \\
24 & 98.1 & 4.71 & 4.72 \\
\hline
\end{tabular}

Table 6.33. Supernate Na Analysis from PEP Parallel Test B Caustic Leach

\begin{tabular}{cccc}
\hline $\begin{array}{c}\text { Time } \\
(\mathrm{hrs})\end{array}$ & $\begin{array}{c}\text { Temperature } \\
\left({ }^{\circ} \mathrm{C}\right)\end{array}$ & $\begin{array}{c}\text { B-LAB_CL_1 } \\
(\mathrm{mg} \mathrm{Na} / \mathrm{kg} \mathrm{soln})\end{array}$ & $\begin{array}{c}\text { B-LAB_CL_2 } \\
(\mathrm{mg} \mathrm{Na} / \mathrm{kg} \mathrm{soln})\end{array}$ \\
\hline Initial & 23.4 & 133,000 & 135,000 \\
-0.95 & 88.2 & 134,000 & 137,000 \\
0 & 98.2 & 135,000 & 138,000 \\
1 & 98.0 & 138,000 & 135,000 \\
2 & 98.0 & 136,000 & 137,000 \\
4 & 98.0 & 137,000 & 143,000 \\
8 & 98.0 & 138,000 & 144,000 \\
10 & 97.7 & 138,000 & 141,000 \\
12 & 98.0 & 138,000 & 141,000 \\
14 & 98.0 & 138,000 & 139,000 \\
16 & 98.2 & 138,000 & 142,000 \\
18 & 97.9 & 137,000 & 142,000 \\
21 & 98.0 & 139,000 & 142,000 \\
24 & 98.1 & 140,000 & 144,000 \\
\hline
\end{tabular}


Table 6.34. Slurry Analysis from PEP Parallel Test B Caustic Leach Test 1

\begin{tabular}{|c|c|c|c|c|c|c|}
\hline & $\begin{array}{c}\text { B_LAB_CL_1_SS1_SL } \\
(\mathrm{mg} / \mathrm{kg} \text { slurry })\end{array}$ & $\begin{array}{c}\text { B_LAB_CL_1_SS2_SL } \\
(\mathrm{mg} / \mathrm{kg} \text { slurry })\end{array}$ & $\begin{array}{l}\text { AB_CL_1_SS3 } \\
(\mathrm{mg} / \mathrm{kg} \text { slurry) }\end{array}$ & $\begin{array}{c}\text { B_LAB_CL_1_FS1_SL } \\
(\mathrm{mg} / \mathrm{kg} \text { slurry })\end{array}$ & $\begin{array}{c}\text { B_LAB_CL_1_FS2_SL } \\
(\mathrm{mg} / \mathrm{kg} \text { slurry })\end{array}$ & $\begin{array}{l}\text { LAB_CL_1_FS3_SL } \\
\text { (mg/kg slurry) }\end{array}$ \\
\hline & Initial Washed Slurry-1 & Initial Washed Slurry-2 & Initial Slurry & Final Washed Slurry-1 & Final Washed Slurry-2 & Final Slurry \\
\hline $\mathrm{Al}$ & 37,800 & 39,400 & 26,800 & 29,500 & 27,700 & 28,300 \\
\hline $\mathrm{Ca}$ & 758 & 768 & 286 & 905 & 888 & 308 \\
\hline $\mathrm{Cr}$ & 29.3 & 22 & 16 & 31.8 & 31.8 & 15.5 \\
\hline $\mathrm{Fe}$ & 23,500 & 24,200 & 8,960 & 28,100 & 27,900 & 9,660 \\
\hline $\mathrm{Mg}$ & 454 & 469 & 169 & 534 & 525 & 182 \\
\hline $\mathrm{Mn}$ & 5,260 & 5,310 & 1,940 & 6,370 & 5,950 & 2,090 \\
\hline $\mathrm{Ni}$ & 760 & 784 & 281 & 897 & 887 & 304 \\
\hline $\mathrm{Na}$ & 2,250 & 1,790 & 132,000 & 2,610 & 2,850 & 142,000 \\
\hline $\mathrm{P}$ & 116 & 125 & 742 & 152 & 155 & 661 \\
\hline $\mathrm{Sr}$ & 209 & 213 & 80.6 & 250 & 244 & 87.2 \\
\hline $\mathrm{Zr}$ & 696 & 697 & 242 & 811 & 824 & 184 \\
\hline
\end{tabular}

i

Table 6.35. Slurry Analysis from PEP Parallel Test B Caustic Leach Test 2

\begin{tabular}{|c|c|c|c|c|c|c|}
\hline & $\begin{array}{c}\text { B_LAB_CL_2_SS1_SL } \\
(\mathrm{mg} / \mathrm{kg} \text { slurry })\end{array}$ & $\begin{array}{c}\text { B_LAB_CL_2_SS2_SL } \\
(\mathrm{mg} / \mathrm{kg} \text { slurry })\end{array}$ & $\begin{array}{c}\text { AB_CL_2_SS3 } \\
(\mathrm{mg} / \mathrm{kg} \text { slurry) }\end{array}$ & $\begin{array}{c}\text { B_LAB_CL_2_FS1_SI } \\
(\mathrm{mg} / \mathrm{kg} \text { slurry })\end{array}$ & $\begin{array}{c}\text { B_LAB_CL_2_FS2_SL } \\
(\mathrm{mg} / \mathrm{kg} \text { slurry })\end{array}$ & $\begin{array}{l}\text { LAB_CL_2_FS3_SL } \\
\text { (mg/kg slurry) }\end{array}$ \\
\hline & Initial Washed Slurry-1 & Initial Washed Slurry-2 & Initial Slurry & Final Washed Slurry-1 & Final Washed Slurry-2 & Final Slurry \\
\hline $\mathrm{Al}$ & 36,700 & 39,500 & 25,700 & 26,800 & 26,300 & 28,700 \\
\hline $\mathrm{Ca}$ & 763 & 749 & 285 & 842 & 846 & 312 \\
\hline $\mathrm{Cr}$ & 25.1 & 24.5 & 14.5 & 31.1 & 33.6 & 15.9 \\
\hline $\mathrm{Fe}$ & 23,900 & 23,500 & 8,850 & 26,300 & 26,400 & 9,730 \\
\hline $\mathrm{Mg}$ & 461 & 451 & 169 & 502 & 500 & 186 \\
\hline $\mathrm{Mn}$ & 5,330 & 5,360 & 1,910 & 5,700 & 5,600 & 2,120 \\
\hline $\mathrm{Ni}$ & 771 & 758 & 276 & 842 & 842 & 307 \\
\hline $\mathrm{Na}$ & 1,820 & 1,760 & 130,000 & 2,760 & 2,930 & 143,000 \\
\hline$P$ & 125 & 126 & 738 & 163 & 162 & 777 \\
\hline $\mathrm{Sr}$ & 214 & 212 & 79.6 & 231 & 232 & 86.6 \\
\hline $\mathrm{Zr}$ & 684 & 688 & 251 & 765 & 777 & 255 \\
\hline
\end{tabular}


Table 6.36. Anion Analysis of B_LAB_CL_1 Supernate

\begin{tabular}{cccccccc}
\hline Time $(\mathrm{hrs})$ & $\begin{array}{c}\text { Temperature } \\
\left({ }^{\circ} \mathrm{C}\right)\end{array}$ & $\begin{array}{c}\text { Chloride } \\
(\mathrm{mg} / \mathrm{kg} \text { soln })\end{array}$ & $\begin{array}{c}\text { Nitrate } \\
(\mathrm{mg} / \mathrm{kg} \text { soln })\end{array}$ & $\begin{array}{c}\text { Nitrite } \\
(\mathrm{mg} / \mathrm{kg} \text { soln })\end{array}$ & $\begin{array}{c}\text { Oxalate } \\
(\mathrm{mg} / \mathrm{kg} \text { soln })\end{array}$ & $\begin{array}{c}\text { Phosphate } \\
(\mathrm{mg} / \mathrm{kg} \mathrm{soln})\end{array}$ & $\begin{array}{c}\text { Sulfate } \\
(\mathrm{mg} / \mathrm{kg} \text { soln })\end{array}$ \\
\hline Initial & 23.4 & 414 & 34,000 & 8,150 & 77.2 & 2,080 & 6,590 \\
-0.95 & 88.2 & 409 & 34,100 & 8,080 & 271 & 2,120 & 6,540 \\
0 & 98.2 & 422 & 34,500 & 8,150 & 490 & 2,280 & 6,690 \\
1 & 98.0 & 429 & 35,100 & 8,350 & 466 & 2,300 & 6,780 \\
2 & 98.0 & 418 & 34,500 & 8,210 & 466 & 2,270 & 6,690 \\
4 & 98.0 & 427 & 35,000 & 8,350 & 458 & 2,240 & 6,760 \\
8 & 98.0 & 428 & 34,700 & 8,310 & 473 & 2,190 & 6,670 \\
10 & 97.7 & 429 & 35,400 & 8,350 & 321 & 2,240 & 6,770 \\
12 & 98.0 & 432 & 35,500 & 8,440 & 289 & 2,120 & 6,730 \\
14 & 98.0 & 426 & 35,000 & 8,310 & 281 & 2,050 & 6,660 \\
16 & 98.2 & 430 & 35,800 & 8,480 & 138 & 2,080 & 6,810 \\
18 & 97.9 & 438 & 35,900 & 8,480 & 158 & 2,130 & 6,860 \\
21 & 98.0 & 438 & 36,300 & 8,480 & 119 & 1,930 & 6,800 \\
24 & 98.1 & 443 & 36,200 & 8,640 & 113 & 2,000 & 6,780 \\
\hline
\end{tabular}

Table 6.37. Anion Analysis of B_LAB_CL_2 Supernate

\begin{tabular}{cccccccc}
\hline Time $(\mathrm{hrs})$ & $\begin{array}{c}\text { Temperature } \\
\left({ }^{\circ} \mathrm{C}\right)\end{array}$ & $\begin{array}{c}\text { Chloride } \\
(\mathrm{mg} / \mathrm{kg} \text { soln })\end{array}$ & $\begin{array}{c}\text { Nitrate } \\
(\mathrm{mg} / \mathrm{kg} \text { soln })\end{array}$ & $\begin{array}{c}\text { Nitrite } \\
(\mathrm{mg} / \mathrm{kg} \text { soln })\end{array}$ & $\begin{array}{c}\text { Oxalate } \\
(\mathrm{mg} / \mathrm{kg} \text { soln })\end{array}$ & $\begin{array}{c}\text { Phosphate } \\
(\mathrm{mg} / \mathrm{kg} \mathrm{soln})\end{array}$ & $\begin{array}{c}\text { Sulfate } \\
(\mathrm{mg} / \mathrm{kg} \text { soln })\end{array}$ \\
\hline Initial & 23.4 & 408 & 33,400 & 7,980 & 69.7 & 1,700 & 6,560 \\
-0.95 & 88.2 & 419 & 34,100 & 8,080 & 283 & 2,150 & 6,530 \\
0 & 98.2 & 426 & 35,000 & 8,450 & 448 & 2,150 & 6,600 \\
1 & 98.0 & 422 & 34,500 & 8,210 & 457 & 2,300 & 6,700 \\
2 & 98.0 & 418 & 34,500 & 8,210 & 388 & 2,250 & 6,630 \\
4 & 98.0 & 456 & 37,500 & 8,670 & 437 & 2,270 & 7,020 \\
8 & 98.0 & 455 & 36,900 & 8,670 & 443 & 2,180 & 6,940 \\
10 & 97.7 & 435 & 35,500 & 8,480 & 315 & 2,140 & 6,810 \\
12 & 98.0 & 439 & 35,200 & 8,310 & 301 & 2,050 & 6,670 \\
14 & 98.0 & 432 & 34,900 & 8,380 & 291 & 2,030 & 6,670 \\
16 & 98.2 & 432 & 35,400 & 8,380 & 192 & 2,000 & 6,700 \\
18 & 97.9 & 433 & 35,500 & 8,380 & 163 & 1,900 & 6,650 \\
21 & 98.0 & 439 & 36,000 & 8,510 & 207 & 1,900 & 6,760 \\
24 & 98.1 & 438 & 35,900 & 8,640 & 141 & 2,020 & 6,690 \\
\hline
\end{tabular}


Table 6.38. Anion Analysis of Slurry Samples for Test B Caustic Leach

\begin{tabular}{|c|c|c|c|c|c|c|c|}
\hline Sample ID & $\begin{array}{c}\text { Sample } \\
\text { Description }\end{array}$ & $\begin{array}{c}\text { Chloride } \\
\text { (mg/kg } \\
\text { slurry) }\end{array}$ & $\begin{array}{l}\text { Nitrate } \\
(\mathrm{mg} / \mathrm{kg} \\
\text { slurry })\end{array}$ & $\begin{array}{l}\text { Nitrite } \\
(\mathrm{mg} / \mathrm{kg} \\
\text { slurry })\end{array}$ & $\begin{array}{l}\begin{array}{l}\text { Oxalate } \\
(\mathrm{mg} / \mathrm{kg} \\
\text { slurry })\end{array} \\
\end{array}$ & $\begin{array}{c}\text { Phosphate } \\
\text { (mg/kg } \\
\text { slurry) }\end{array}$ & $\begin{array}{c}\text { Sulfate } \\
(\mathrm{mg} / \mathrm{kg} \\
\text { slurry) }\end{array}$ \\
\hline B_LAB_CL_1_SS1_SL & $\begin{array}{c}\text { Test } 1 \text { Initial } \\
\text { Washed } \\
\text { Slurry -1 }\end{array}$ & $<18.6$ & 137 & $<61$ & 106 & 101 & 28.2 \\
\hline B_LAB_CL_1_SS2_SL & $\begin{array}{c}\text { Test } 1 \text { Initial } \\
\text { Washed } \\
\text { Slurry -2 }\end{array}$ & $<19.0$ & 107 & $<62$ & 68.6 & 101 & 23.3 \\
\hline B_LAB_CL_1_SS3_SL & $\begin{array}{c}\text { Test } 1 \text { Initial } \\
\text { Slurry }-3\end{array}$ & 334 & 28,000 & 7,130 & 7,860 & 1,980 & 5,580 \\
\hline B_LAB_CL_1_FS1_SL & $\begin{array}{c}\text { Test } 1 \text { Final } \\
\text { Washed } \\
\text { Slurry -1 }\end{array}$ & $<19.8$ & 148 & $<65$ & 224 & 186 & 43.2 \\
\hline B_LAB_CL_1_FS2_SL & $\begin{array}{c}\text { Test } 1 \text { Final } \\
\text { Washed } \\
\text { Slurry -2 }\end{array}$ & $<19.4$ & 139 & $<64$ & 1,040 & 169 & 30.8 \\
\hline B_LAB_CL_1_FS3_SL & $\begin{array}{c}\text { Test } 1 \text { Final } \\
\text { Slurry }-3\end{array}$ & 369 & 30,500 & 7,660 & 8,450 & 1,940 & 6,010 \\
\hline B_LAB_CL_2_SS1_SL & $\begin{array}{c}\text { Test } 1 \text { Initial } \\
\text { Washed } \\
\text { Slurry }-1\end{array}$ & $<19.3$ & 96 & $<63$ & 57.1 & 108 & $<19.3$ \\
\hline B_LAB_CL_2_SS2_SL & $\begin{array}{c}\text { Test } 1 \text { Initial } \\
\text { Washed } \\
\text { Slurry -2 }\end{array}$ & $<18.6$ & 98 & $<61$ & 61.7 & 105 & $<18.6$ \\
\hline B_LAB_CL_2_SS3_SL & $\begin{array}{c}\text { Test } 1 \text { Initial } \\
\text { Slurry }-3\end{array}$ & 342 & 28,200 & 7,100 & 8,050 & 2,040 & 5,520 \\
\hline B_LAB_CL_2_FS1_SL & $\begin{array}{c}\text { Test } 2 \text { Final } \\
\text { Washed } \\
\text { Slurry }-1\end{array}$ & $<18.8$ & 162 & $<62$ & 650 & 184 & 52.6 \\
\hline B_LAB_CL_2_FS2_SL & $\begin{array}{c}\text { Test } 2 \text { Final } \\
\text { Washed } \\
\text { Slurry }-2\end{array}$ & $<19.3$ & 188 & 64 & 676 & 207 & 68.2 \\
\hline B_LAB_CL_2_FS3_SL & $\begin{array}{c}\text { Test } 2 \text { Final } \\
\text { Slurry }-3\end{array}$ & 371 & 30,600 & 7,660 & 8,700 & 1,640 & 6,070 \\
\hline
\end{tabular}


Table 6.39. PEP Parallel Test B Free OH Concentration

\begin{tabular}{cccc}
\hline Sample ID & OH Conc. (mol/L) & Sample ID & OH Conc. (mol/L) \\
\hline B_LAB_CL_1_000 & 5.93 & B_LAB_CL_2_000 & 5.42 \\
B_LAB_CL_1_001 & 5.67 & B_LAB_CL_2_001 & 5.47 \\
B_LAB_CL_1_002 & 5.70 & B_LAB_CL_2_002 & 5.48 \\
B_LAB_CL_1_004 & 5.50 & B_LAB_CL_2_004 & 5.46 \\
B_LAB_CL_1_008 & 5.62 & B_LAB_CL_2_008 & 5.67 \\
B_LAB_CL_1_010 & 5.59 & B_LAB_CL_2_010 & 5.53 \\
B_LAB_CL_1_012 & 5.43 & B_LAB_CL_2_012 & 5.58 \\
B_LAB_CL_1_014 & 5.65 & B_LAB_CL_2_014 & 5.39 \\
B_LAB_CL_1_016 & 5.80 & B_LAB_CL_2_016 & 5.67 \\
B_LAB_CL_1_018 & 5.58 & B_LAB_CL_2_018 & 5.59 \\
B_LAB_CL_1_021 & 4.67 & B_LAB_CL_2_021 & 5.47 \\
B_LAB_CL_1_024 & 5.62 & B_LAB_CL_2_024 & 5.46 \\
\hline
\end{tabular}

Table 6.40. PEP Parallel Test B Physical Properties for Test 1

\begin{tabular}{lccc}
\hline \multicolumn{1}{c}{ Property } & B_LAB_CL_1_SS1_SL & B_LAB_CL_1_SS2_SL & B_LAB_CL_1_SS3_SL \\
\hline Sample Description & Initial Washed Slurry-1 & Initial Washed Slurry-2 & Initial Slurry \\
\hline Bulk Density (g/mL) & 1.146 & 1.103 & 1.370 \\
Density of Cent. Solids $(\mathrm{g} / \mathrm{mL})$ & 1.546 & 1.568 & 1.812 \\
Vol\% of Cent. Solids & 26.5 & 25.5 & 18.0 \\
Wt\% Cent. Solids & 35.8 & 36.2 & 23.8 \\
Supernate Density (g/mL) & 0.989 & 0.934 & 1.270 \\
Wt\% Total Solids & 15.8 & 16.0 & 38.0 \\
Wt\% Oven Dried Solids & 43.5 & 43.7 & 50.2 \\
Wt\% UDS & 15.5 & 15.8 & 5.74 \\
Wt\% Dissolved Solids & 0.373 & 0.225 & 34.3 \\
Wt\% Supernate Liquid & 63.4 & 63.1 & 76.0 \\
\hline
\end{tabular}

Table 6.40 (cont)

\begin{tabular}{lccc}
\hline \multicolumn{1}{c}{ Property } & B_LAB_CL_1_FS1_SL & B_LAB_CL_1_FS2_SL & B_LAB_CL_1_FS3_SL \\
\hline Sample Description & Final Washed Slurry-1 & Final Washed Slurry-2 & Final Slurry \\
Bulk Density $(\mathrm{g} / \mathrm{mL})$ & 1.090 & 1.113 & 1.426 \\
Density of Cent. Solids $(\mathrm{g} / \mathrm{mL})$ & 1.399 & 1.475 & 1.995 \\
Vol\% of Cent. Solids & 24.5 & 23.4 & 18.4 \\
Wt\% Cent. Solids & 31.4 & 31.0 & 25.7 \\
Supernate Density $(\mathrm{g} / \mathrm{mL})$ & 0.984 & 0.996 & 1.296 \\
Wt\% Total Solids & 12.6 & 11.6 & 40.3 \\
Wt\% Oven Dried Solids & 39.5 & 36.3 & 49.4 \\
Wt\% UDS & 12.3 & 11.2 & 5.01 \\
Wt\% Dissolved Solids & 0.341 & 0.488 & 37.2 \\
Wt\% Supernate Liquid & 68.2 & 68.5 & 74.2 \\
\hline
\end{tabular}


Table 6.41. PEP Parallel Test B Physical Properties for Test 2

\begin{tabular}{lccc}
\hline \multicolumn{1}{c}{ Property } & B_LAB_CL_2_SS1_SL & B_LAB_CL_2_SS2_SL & B_LAB_CL_2_SS3_SL \\
\hline Sample Description & Initial Washed Slurry-1 & Initial Washed Slurry-2 & Initial Slurry \\
Bulk Density $(\mathrm{g} / \mathrm{mL})$ & 1.150 & 1.148 & 1.402 \\
Density of Cent. Solids $(\mathrm{g} / \mathrm{mL})$ & 1.559 & 1.532 & 1.855 \\
Vol\% of Cent. Solids & 26.5 & 26.5 & 18.4 \\
Wt\% Cent. Solids & 36.0 & 35.4 & 24.3 \\
Supernate Density $(\mathrm{g} / \mathrm{mL})$ & 0.994 & 1.002 & 1.298 \\
Wt $\%$ Total Solids & 15.7 & 15.8 & 37.7 \\
Wt\% Oven Dried Solids & 43.5 & 44.2 & 49.4 \\
Wt\% UDS & 15.6 & 15.6 & 5.67 \\
Wt $\%$ Dissolved Solids & 0.120 & 0.180 & 34.0 \\
Wt $\%$ Supernate Liquid & 63.5 & 64.1 & 75.6 \\
\hline
\end{tabular}

Table 6.41 (cont)

\begin{tabular}{lccc}
\hline \multicolumn{1}{c}{ Property } & B_LAB_CL_2_FS1_SL & B_LAB_CL_2_FS2_SL & B_LAB_CL_2_FS3_SL \\
\hline Sample Description & Final Washed Slurry-1 & Final Washed Slurry-2 & Final Slurry \\
Bulk Density $(\mathrm{g} / \mathrm{mL})$ & 1.100 & 1.104 & 1.430 \\
Density of Cent. Solids (g/mL) & 1.408 & 1.467 & 2.065 \\
Vol\% of Cent. Solids & 25.0 & 23.4 & 18.4 \\
Wt\% Cent. Solids & 32.0 & 31.1 & 26.5 \\
Supernate Density (g/mL) & 0.991 & 0.985 & 1.284 \\
Wt\% Total Solids & 12.7 & 12.1 & 40.2 \\
Wt\% Oven Dried Solids & 38.9 & 38.0 & 48.6 \\
Wt\% UDS & 12.4 & 11.7 & 4.80 \\
Wt\% Dissolved Solids & 0.423 & 0.437 & 37.2 \\
Wt\% Supernate Liquid & 67.5 & 68.3 & 73.3 \\
\hline
\end{tabular}

\subsection{PEP Test D Caustic Leach}

The caustic leach process for PEP Test D was conducted under Test Instruction TI-WTP-PEP-082 (TI-082) in the PEP UFP-VSL-T02A tank and was completed as follows. Feed stored in HLP-VSL-T22 was transferred into vessel UFP-VSL-T01A. AFA was added directly to UFP-VSL-T01A in a quantity expected to produce a nominal concentration of $350 \mathrm{ppm}$ in the slurry. The slurry was then transferred from the UFP-VSL-T01A feed tank to UFP-VSL-T02A, and permeate was removed from UFP-VSL-T02A through the first ultrafilter bundle to increase the solids concentration of the slurry. As permeate was removed, the volume (and level) in UFP-VSL-T02A fell, triggering the transfer of small refill batches (i.e., $11 \mathrm{gal}$ ) of fresh simulant from UFP-VSL-T01A. The filtering and refill process continued, leaving a target quantity of slurry at about 20 -wt $\%$ UDS in the UFP-VSL-T02A vessel and filter loop. When the solids concentration process was complete, the permeate valves were closed on the filter system, and caustic reagent was introduced upstream of the filter loop pumps.

The caustic slurry in UFP-VSL-T02A was heated to about $70^{\circ} \mathrm{C}$ using the heat of dilution of the concentrated $\mathrm{NaOH}$ and mechanical heat from the filter loop recirculation pumps. A sample of slurry was taken from the middle-low region of vessel UFP-VSL-T02A using the CD sampler for use as feed in the 
laboratory-scale caustic leach tests (D_LAB_CL_1 and D_LAB_CL_2 as duplicates) on March 27, 2009 at 16:07. The sample was rapidly cooled in a cold water bath to ambient temperature to minimize any further leaching reaction.

The laboratory-scale feed was stored at laboratory ambient temperature (approximately $20^{\circ} \mathrm{C}$ to $25^{\circ} \mathrm{C}$ ) until it was used. The delay between the time when the feed was acquired from PEP and the time laboratory-scale testing started was about 12 days for Test D (from March 27, 2009, to April 8, 2009).

The PEP sample ID, laboratory sample ID, and the amount of water used in the parallel laboratory-scale tests are shown in Table 6.42.

Table 6.42. PEP Parallel Test D Matrix

\begin{tabular}{cccc}
\hline & & PEP Sample & DI Water \\
PEP Sample ID & Test ID & $(\mathrm{g})$ & $(\mathrm{g})$ \\
\hline D_02AML_07A_XX_5198_CUF_4 & D_LAB_CL_1 & 685.02 & 115.01 \\
D_02AML_07A_XX_5198_CUF_4 & D_LAB_CL_2 & 685.01 & 115.01 \\
\hline
\end{tabular}

These tests were performed by placing the required amount of PEP simulant and DI water in the test vessel, attaching the lid, and heating to $70^{\circ} \mathrm{C}$ while stirring. At $70^{\circ} \mathrm{C}$, the vessel contents were heated at a linear rate to $85 \pm 2{ }^{\circ} \mathrm{C}$ over a 1 hour and 35 minute time span. When the vessel reached $75 \pm 2^{\circ} \mathrm{C}$, a sample was taken as the $-1.53 \mathrm{hr}$ sample. At $85 \pm 2{ }^{\circ} \mathrm{C}$, the time zero $(0 \mathrm{hr})$ sample was taken, and the test was held for 24 hours at this temperature. Samples were taken at 1, 2, 4, 8, 10, 12, 14, 16, 18, and 24 hours and immediately filtered through a $0.45-\mu \mathrm{m}$ syringe filter. These samples were then analyzed by ICP-AES for Al, Cr, and Na concentration at SWRI, and the results are shown in Table 6.43 through Table 6.45. Slurry samples were also taken at the beginning and end of the test. Some slurry samples were washed three times with $0.01 \mathrm{M} \mathrm{NaOH}$ and others were not washed. These results are shown in Table 6.46 and Table 6.47. The samples were analyzed by IC for anion concentrations, which are shown in Table 6.48 and Table 6.49 for the supernate and Table 6.50 for the slurries. The $\mathrm{OH}^{-}$concentration was also measured on the supernates and is shown in Table 6.51. The wt\% UDS along with other physical properties were measured on the slurries (both washed and unwashed) and are shown in Table 6.52 and Table 6.53 . 
Table 6.43. Supernate Al Analysis from PEP Parallel Test D Caustic Leach

\begin{tabular}{cccc}
\hline $\begin{array}{c}\text { Time } \\
(\mathrm{hrs})\end{array}$ & $\begin{array}{c}\text { Temperature } \\
\left({ }^{\circ} \mathrm{C}\right)\end{array}$ & $\begin{array}{c}\text { D_LAB_CL_1 } \\
(\mathrm{mg} \text { A1/kg soln })\end{array}$ & $\begin{array}{c}\text { D_LAB_CL_2 } \\
(\mathrm{mg} \text { A1/kg soln })\end{array}$ \\
\hline Initial & 23.8 & 9,340 & 10,400 \\
-1.53 & 71.8 & 10,400 & 10,700 \\
0 & 85.4 & 10,200 & 10,500 \\
1 & 85.1 & 10,600 & 10,900 \\
2 & 85.0 & 10,700 & 10,900 \\
4 & 85.1 & 11,500 & 11,500 \\
8 & 85.0 & 12,500 & 11,700 \\
10 & 85.0 & 12,700 & 12,500 \\
12 & 84.9 & 13,000 & 13,400 \\
14 & 85.0 & 13,600 & 13,700 \\
16 & 85.0 & 13,900 & 14,000 \\
18 & 85.0 & 14,400 & 14,600 \\
20 & 84.9 & 14,200 & 14,300 \\
24 & 85.0 & 14,900 & 15,000 \\
Final & 31.6 & 14,700 & 14,200 \\
\hline
\end{tabular}

Table 6.44. Supernate Cr Analysis from PEP Parallel Test D Caustic Leach

\begin{tabular}{cccc}
\hline $\begin{array}{c}\text { Time } \\
(\mathrm{hrs})\end{array}$ & $\begin{array}{l}\text { Temperature } \\
\left({ }^{\circ} \mathrm{C}\right)\end{array}$ & $\begin{array}{l}\text { D_LAB_CL_1 } \\
(\mathrm{mg} \text { Cr/kg soln })\end{array}$ & $\begin{array}{l}\text { D_LAB_CL_2 } \\
(\mathrm{mg} \text { Cr/kg soln })\end{array}$ \\
\hline Initial & 23.8 & 169 & 179 \\
-1.53 & 71.8 & 195 & 199 \\
0 & 85.4 & 307 & 307 \\
1 & 85.1 & 368 & 362 \\
2 & 85.0 & 419 & 414 \\
4 & 85.1 & 497 & 492 \\
8 & 85.0 & 591 & 552 \\
10 & 85.0 & 614 & 615 \\
12 & 84.9 & 650 & 654 \\
14 & 85.0 & 685 & 686 \\
16 & 85.0 & 719 & 721 \\
18 & 85.0 & 757 & 758 \\
20 & 84.9 & 741 & 771 \\
24 & 85.0 & 803 & 821 \\
Final & 31.6 & 787 & 787 \\
\hline
\end{tabular}


Table 6.45. Supernate Na Analysis from PEP Parallel Test D Caustic Leach

\begin{tabular}{cccc}
\hline $\begin{array}{c}\text { Time } \\
(\mathrm{hrs})\end{array}$ & $\begin{array}{c}\text { Temperature } \\
\left({ }^{\circ} \mathrm{C}\right)\end{array}$ & $\begin{array}{c}\text { D_LAB_CL_1 } \\
(\mathrm{mg} \mathrm{Na} / \mathrm{kg} \text { soln })\end{array}$ & $\begin{array}{c}\text { D_LAB_CL_2 } \\
(\mathrm{mg} \mathrm{Na} / \mathrm{kg} \text { soln })\end{array}$ \\
\hline Initial & 23.8 & 154,000 & 161,000 \\
-1.53 & 71.8 & 162,000 & 166,000 \\
0 & 85.4 & 166,000 & 166,000 \\
1 & 85.1 & 166,000 & 166,000 \\
2 & 85.0 & 168,000 & 166,000 \\
4 & 85.1 & 172,000 & 174,000 \\
8 & 85.0 & 179,000 & 164,000 \\
10 & 85.0 & 175,000 & 172,000 \\
12 & 84.9 & 174,000 & 175,000 \\
14 & 85.0 & 176,000 & 174,000 \\
16 & 85.0 & 177,000 & 177,000 \\
18 & 85.0 & 179,000 & 176,000 \\
20 & 84.9 & 174,000 & 168,000 \\
24 & 85.0 & 171,000 & 168,000 \\
Final & 31.6 & 165,000 & 162,000 \\
\hline
\end{tabular}

Table 6.46. Slurry Analysis from PEP Parallel Test D Caustic Leach Test 1

\begin{tabular}{|c|c|c|c|c|c|c|}
\hline & $\begin{array}{c}\text { D_LAB_- } \\
\text { CL_1_IS_SL } \\
\text { (mg/kg slurry) }\end{array}$ & $\begin{array}{c}\text { D_LAB_ } \\
\text { CL_1_ISW1_SL } \\
\text { (mg/kg slurry) }\end{array}$ & $\begin{array}{c}\text { D_LAB_ } \\
\text { CL_1_ISW2_SL } \\
\text { (mg/kg slurry) }\end{array}$ & $\begin{array}{l}\text { D_LAB_- } \\
\text { CL_1_FS1_SL } \\
\text { (mg/kg slurry) }\end{array}$ & $\begin{array}{c}\text { D_LAB_ } \\
\text { CL_1_FSW1_SL } \\
\text { (mg/kg slurry) }\end{array}$ & $\begin{array}{c}\text { D_LAB_ } \\
\text { CL_1_FSW2_SL } \\
\text { (mg/kg slurry) }\end{array}$ \\
\hline & Initial Slurry & $\begin{array}{c}\text { Initial Washed } \\
\text { Slurry-1 }\end{array}$ & $\begin{array}{l}\text { Initial Washed } \\
\text { Slurry-2 }\end{array}$ & Final Slurry & $\begin{array}{c}\text { Final Washed } \\
\text { Slurry-1 }\end{array}$ & $\begin{array}{c}\text { Final Washed } \\
\text { Slurry-2 }\end{array}$ \\
\hline $\mathrm{Al}$ & 22,100 & 41,000 & 39,100 & 25,200 & 33,900 & 30,000 \\
\hline $\mathrm{Ca}$ & 237 & 391 & 513 & 249 & 630 & 627 \\
\hline $\mathrm{Cr}$ & 1,260 & 1,490 & 2,200 & 1,360 & 1,470 & 1,500 \\
\hline $\mathrm{Fe}$ & 7,670 & 11,200 & 16,000 & 8,160 & 20,300 & 20,800 \\
\hline $\mathrm{Mg}$ & 146 & 257 & 323 & 154 & 385 & 389 \\
\hline $\mathrm{Mn}$ & 1,570 & 3,770 & 4,110 & 1,550 & 4,000 & 3,920 \\
\hline $\mathrm{Ni}$ & 241 & 398 & 526 & 256 & 639 & 655 \\
\hline $\mathrm{Na}$ & 150,000 & 1,310 & 1,470 & 162,000 & 2,420 & 4,390 \\
\hline $\mathrm{P}$ & 557 & 26.3 & 39.1 & 588 & 41.0 & 47.8 \\
\hline $\mathrm{Sr}$ & 69.2 & 102 & 143 & 74.0 & 177 & 180 \\
\hline $\mathrm{Zr}$ & 178 & 313 & 436 & 190 & 552 & 560 \\
\hline
\end{tabular}


Table 6.47. Slurry Analysis from PEP Parallel Test D Caustic Leach Test 2

\begin{tabular}{|c|c|c|c|c|c|c|}
\hline & $\begin{array}{l}\text { D_LAB_- } \\
\text { CL_2_IS_SL } \\
\text { (mg/kg slurry) }\end{array}$ & $\begin{array}{c}\text { D_LAB_- } \\
\text { CL_2_ISW1_SL } \\
\text { (mg/kg slurry) }\end{array}$ & $\begin{array}{c}\text { D_LAB_- } \\
\text { CL_2_ISW2_SL } \\
\text { (mg/kg slurry) }\end{array}$ & $\begin{array}{l}\text { D_LAB_- } \\
\text { CL_2_FS1_SL } \\
\text { (mg/kg slurry) }\end{array}$ & $\begin{array}{c}\text { D_LAB_- } \\
\text { CL_2_FSW1_SL } \\
(\mathrm{mg} / \mathrm{kg} \text { slurry) }\end{array}$ & $\begin{array}{c}\text { D_LAB_- } \\
\text { CL_2_FSW2_SL } \\
(\mathrm{mg} / \mathrm{kg} \text { slurry) }\end{array}$ \\
\hline & Initial Slurry & Initial Washed Slurry-1 & Initial Washed Slurry-2 & Final Slurry & Final Washed Slurry-1 & Final Washed Slurry-2 \\
\hline $\mathrm{Al}$ & 22,100 & 36,900 & 39,200 & 24,500 & 29,100 & 31,100 \\
\hline $\mathrm{Ca}$ & 235 & 376 & 532 & 247 & 639 & 635 \\
\hline $\mathrm{Cr}$ & 1,270 & 1,440 & 2,210 & 1,340 & 1,500 & 1,520 \\
\hline $\mathrm{Fe}$ & 7,730 & 10,800 & 16,100 & 8,010 & 21,200 & 21,400 \\
\hline $\mathrm{Mg}$ & 144 & 247 & 335 & 154 & 405 & 395 \\
\hline $\mathrm{Mn}$ & 1,590 & 3,430 & 4,120 & 1,580 & 3,910 & 4,000 \\
\hline $\mathrm{Ni}$ & 243 & 379 & 530 & 251 & 665 & 674 \\
\hline $\mathrm{Na}$ & 149,000 & 1,280 & 1,510 & 156,000 & 2,680 & 3,850 \\
\hline $\mathrm{P}$ & 538 & 24.4 & 34.2 & 576 & 41.9 & 51.5 \\
\hline $\mathrm{Sr}$ & 68.7 & 98.1 & 146 & 70.8 & 184 & 187 \\
\hline $\mathrm{Zr}$ & 175 & 297 & 445 & 191 & 573 & 576 \\
\hline
\end{tabular}

Table 6.48. Anion Analysis of D_LAB_CL_1 Supernate

\begin{tabular}{|c|c|c|c|c|c|c|c|}
\hline $\begin{array}{l}\text { Time } \\
\text { (hrs) }\end{array}$ & $\begin{array}{c}\text { Temperature } \\
\left({ }^{\circ} \mathrm{C}\right)\end{array}$ & Chloride ( $\mathrm{mg} / \mathrm{kg}$ soln) & Nitrate (mg/kg soln) & Nitrite (mg/kg soln) & Oxalate (mg/kg soln) & Phosphate (mg/kg soln) & Sulfate $(\mathrm{mg} / \mathrm{kg}$ soln $)$ \\
\hline Initial & 23.8 & 328 & 26,000 & 6,340 & 31.4 & 1,650 & 2,740 \\
\hline-1.53 & 71.8 & 394 & 32,600 & 7,790 & 99.2 & 2,220 & 3,910 \\
\hline 0 & 85.4 & 332 & 27,300 & 6,600 & 174 & 1,860 & 3,640 \\
\hline 1 & 85.1 & 337 & 27,600 & 6,600 & 175 & 1,890 & 3,590 \\
\hline 2 & 85.0 & 340 & 27,300 & 6,740 & 159 & 1,890 & 3,650 \\
\hline 4 & 85.1 & 349 & 28,100 & 6,770 & 184 & 1,900 & 3,570 \\
\hline 8 & 85.0 & 373 & 29,900 & 7,160 & 174 & 2,030 & 3,510 \\
\hline 10 & 85.0 & 359 & 29,100 & 7,100 & 169 & 2,040 & 3,470 \\
\hline 12 & 84.9 & 362 & 29,000 & 7,100 & 123 & 2,030 & 3,320 \\
\hline 14 & 85.0 & 351 & 28,700 & 7,060 & 121 & 2,020 & 3,220 \\
\hline 16 & 85.0 & 372 & 29,900 & 7,200 & 93.6 & 2,090 & 3,120 \\
\hline 18 & 85.0 & 370 & 30,200 & 7,260 & 70.7 & 2,070 & 2,980 \\
\hline 20 & 84.9 & 384 & 31,100 & 7,430 & 37.0 & 2,140 & 2,810 \\
\hline 24 & 85.0 & 379 & 30,600 & 7,430 & 75.4 & 2,060 & 2,920 \\
\hline Final & 31.6 & 389 & 31,000 & 7,390 & 14.9 & 1,580 & 2,310 \\
\hline
\end{tabular}


Table 6.49. Anion Analysis of D_LAB_CL_2 Supernate

\begin{tabular}{cccccccc}
\hline Time $(\mathrm{hrs})$ & $\begin{array}{c}\text { Temperature } \\
\left({ }^{\circ} \mathrm{C}\right)\end{array}$ & $\begin{array}{c}\text { Chloride } \\
(\mathrm{mg} / \mathrm{kg} \text { soln })\end{array}$ & $\begin{array}{c}\text { Nitrate } \\
(\mathrm{mg} / \mathrm{kg} \text { soln })\end{array}$ & $\begin{array}{c}\text { Nitrite } \\
(\mathrm{mg} / \mathrm{kg} \text { soln })\end{array}$ & $\begin{array}{c}\text { Oxalate } \\
(\mathrm{mg} / \mathrm{kg} \text { soln })\end{array}$ & $\begin{array}{c}\text { Phosphate } \\
(\mathrm{mg} / \mathrm{kg} \text { soln })\end{array}$ & $\begin{array}{c}\text { Sulfate } \\
(\mathrm{mg} / \mathrm{kg} \text { soln })\end{array}$ \\
\hline Initial & 23.8 & 384 & 31,200 & 7,460 & 34.6 & 1,930 & 3,200 \\
-1.53 & 71.8 & 403 & 31,500 & 7,790 & 146 & 2,230 & 4,070 \\
0 & 85.4 & 331 & 26,900 & 6,670 & 190 & 1,900 & 3,790 \\
1 & 85.1 & 349 & 27,600 & 6,670 & 193 & 1,900 & 3,710 \\
2 & 85.0 & 340 & 27,500 & 6,700 & 158 & 1,930 & 3,670 \\
4 & 85.1 & 355 & 27,900 & 6,770 & 208 & 1,940 & 3,750 \\
8 & 85.0 & 342 & 27,700 & 6,770 & 177 & 1,940 & 3,650 \\
10 & 85.0 & 357 & 28,600 & 7,030 & 131 & 2,010 & 3,700 \\
12 & 84.9 & 478 & 29,100 & 6,930 & 97.4 & 2,060 & 3,490 \\
14 & 85.0 & 373 & 29,600 & 7,130 & 130 & 2,040 & 3,610 \\
16 & 85.0 & 359 & 28,700 & 7,030 & 88.0 & 2,000 & 3,560 \\
18 & 85.0 & 358 & 29,000 & 7,100 & 92.9 & 2,020 & 3,520 \\
20 & 84.9 & 371 & 29,500 & 7,200 & 34.3 & 2,050 & 3,240 \\
24 & 85.0 & 359 & 29,000 & 6,930 & 88.2 & 1,980 & 3,230 \\
Final & 31.6 & 318 & 25,000 & 5,950 & 16.3 & 1,280 & 2,220 \\
\hline
\end{tabular}


Table 6.50. Anion Analysis of Slurry Samples for Test D Caustic Leach

\begin{tabular}{|c|c|c|c|c|c|c|c|}
\hline Sample ID & $\begin{array}{c}\text { Sample } \\
\text { Description }\end{array}$ & $\begin{array}{c}\text { Chloride } \\
\text { (mg/kg } \\
\text { slurry) }\end{array}$ & $\begin{array}{l}\begin{array}{l}\text { Nitrate } \\
(\mathrm{mg} / \mathrm{kg} \\
\text { slurry })\end{array} \\
\end{array}$ & $\begin{array}{l}\text { Nitrite } \\
(\mathrm{mg} / \mathrm{kg} \\
\text { slurry) }\end{array}$ & $\begin{array}{l}\text { Oxalate } \\
\text { (mg/kg } \\
\text { slurry) }\end{array}$ & $\begin{array}{c}\text { Phosphate } \\
\text { (mg/kg } \\
\text { slurry) }\end{array}$ & 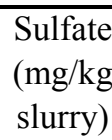 \\
\hline D_LAB_CL_1_IS_SL & $\begin{array}{l}\text { Test } 1 \text { Initial } \\
\text { Slurry }\end{array}$ & 283 & 22,400 & 5,520 & 6,410 & 1,550 & 4,440 \\
\hline D_LAB_CL_1_ISW1_SL & $\begin{array}{c}\text { Test } 1 \text { Initial } \\
\text { Washed } \\
\text { Slurry -1 }\end{array}$ & $<18.6$ & 100 & $<61$ & 150 & $<57$ & 47.4 \\
\hline D_LAB_CL_1_ISW2_SL & $\begin{array}{c}\text { Test } 1 \text { Initial } \\
\text { Washed } \\
\text { Slurry -2 }\end{array}$ & $<19.1$ & 95 & $<63$ & 157 & $<59$ & $<19.1$ \\
\hline D_LAB_CL_1_FS1_SL & $\begin{array}{l}\text { Test } 1 \text { Final } \\
\text { Slurry }-1\end{array}$ & 299 & 23,700 & 5,910 & 6,510 & 1,610 & 4,660 \\
\hline D_LAB_CL_1_FSW1_SL & $\begin{array}{c}\text { Test } 1 \text { Final } \\
\text { Washed } \\
\text { Slurry -2 }\end{array}$ & $<19.9$ & 164 & $<65$ & 392 & $<61$ & 190 \\
\hline D_LAB_CL_1_FSW2_SL & $\begin{array}{c}\text { Test } 1 \text { Final } \\
\text { Washed } \\
\text { Slurry }-3\end{array}$ & $<19.3$ & 366 & 113 & 1,230 & 92 & 365 \\
\hline D_LAB_CL_2_IS_SL & $\begin{array}{l}\text { Test } 2 \text { Initial } \\
\text { Slurry }\end{array}$ & 284 & 22,300 & 5,520 & 6,480 & 1,580 & 4,480 \\
\hline D_LAB_CL_2_ISW1_SL & $\begin{array}{c}\text { Test } 2 \text { Initial } \\
\text { Washed } \\
\text { Slurry -1 }\end{array}$ & $<19.4$ & 103 & $<64$ & 166 & $<59$ & 28.3 \\
\hline D_LAB_CL_2_ISW2_SL & $\begin{array}{c}\text { Test } 2 \text { Initial } \\
\text { Washed } \\
\text { Slurry -2 }\end{array}$ & $<19.4$ & 99 & $<64$ & 158 & $<59$ & $<19.4$ \\
\hline D_LAB_CL_2_FS1_SL & $\begin{array}{l}\text { Test } 2 \text { Final } \\
\text { Slurry }-1\end{array}$ & 292 & 23,100 & 5,750 & 6,360 & 1,560 & 4,460 \\
\hline D_LAB_CL_2_FSW1_SL & $\begin{array}{c}\text { Test } 2 \text { Final } \\
\text { Washed } \\
\text { Slurry -2 }\end{array}$ & $<19.9$ & 183 & 67 & 572 & 67 & 152 \\
\hline D_LAB_CL_2_FSW2_SL & $\begin{array}{c}\text { Test } 2 \text { Final } \\
\text { Washed } \\
\text { Slurry }-3\end{array}$ & $<19.1$ & 331 & 101 & 1,090 & 89 & 260 \\
\hline
\end{tabular}


Table 6.51. PEP Parallel Test D Free $\mathrm{OH}$ Concentration

\begin{tabular}{lclc}
\hline \multicolumn{1}{c}{ Sample ID } & OH Conc. (mol/L) & \multicolumn{1}{c}{ Sample ID } & OH Conc. (mol/L) \\
\hline D_LAB_CL_1_IN_SUP & 8.08 & D_LAB_CL_2_IN_SUP & 7.91 \\
D_LAB_CL_1_010C & 7.86 & D_LAB_CL_2_010C & 8.06 \\
D_LAB_CL_1_000 & 8.11 & D_LAB_CL_2_000 & 8.12 \\
D_LAB_CL_1_001 & 8.03 & D_LAB_CL_2_001 & 8.00 \\
D_LAB_CL_1_002 & 8.13 & D_LAB_CL_2_002 & 8.09 \\
D_LAB_CL_1_004 & 7.99 & D_LAB_CL_2_004 & 8.18 \\
D_LAB_CL_1_008 & 8.17 & D_LAB_CL_2_008 & 7.94 \\
D_LAB_CL_1_010 & 8.34 & D_LAB_CL_2_010 & 8.02 \\
D_LAB_CL_1_012 & 8.35 & D_LAB_CL_2_012 & 8.12 \\
D_LAB_CL_1_014 & 8.41 & D_LAB_CL_2_014 & 8.05 \\
D_LAB_CL_1_016 & 8.13 & D_LAB_CL_2_016 & 8.11 \\
D_LAB_CL_1_018 & 8.36 & D_LAB_CL_2_018 & 8.06 \\
D_LAB_CL_1_020 & 8.42 & D_LAB_CL_2_020 & 7.92 \\
D_LAB_CL_1_024 & 8.21 & D_LAB_CL_2_024 & 8.20 \\
D_LAB_CL_1_F & 8.45 & D_LAB_CL_2_F & 8.54 \\
\hline
\end{tabular}

Table 6.52. PEP Parallel Test D Physical Properties for Test 1

\begin{tabular}{lccc}
\hline \multicolumn{1}{c}{ Property } & D_LAB_CL_1_IS_SL & D_LAB_CL_1_ISW1_SL & D_LAB_CL_1_ISW2_SL \\
\hline Sample Description & Initial Slurry & Initial Washed Slurry-1 & Initial Washed Slurry-2 \\
Bulk Density $(\mathrm{g} / \mathrm{mL})$ & 1.424 & 1.086 & 1.105 \\
Density of Cent. Solids $(\mathrm{g} / \mathrm{mL})$ & 1.729 & 1.519 & 1.447 \\
Vol\% of Cent. Solids & 20.4 & 21.3 & 26.0 \\
Wt\% Cent. Solids & 24.8 & 29.7 & 34.1 \\
Supernate Density $(\mathrm{g} / \mathrm{mL})$ & 1.346 & 0.965 & 0.980 \\
Wt $\%$ Total Solids & 41.5 & 13.7 & 14.4 \\
Wt $\%$ Oven Dried Solids & 50.7 & 46.2 & 42.3 \\
Wt $\%$ UDS & 4.91 & 13.7 & 14.4 \\
W $\%$ Dissolved Solids & 38.5 & 0 & 0.028 \\
Wt $\%$ Supernate Liquid & 75.2 & 70.0 & 65.7 \\
\hline
\end{tabular}

Table 6.52 (cont.)

\begin{tabular}{lccc}
\hline \multicolumn{1}{c}{ Property } & D_LAB_CL_1_FS1_SL & D_LAB_CL_1_FSW1_SL & D_LAB_CL_1_FSW2_SL \\
\hline Sample Description & Final Slurry & Final Washed Slurry-1 & Final Washed Slurry-2 \\
\hline Bulk Density $(\mathrm{g} / \mathrm{mL})$ & 1.408 & 1.081 & 1.090 \\
Density of Cent. Solids $(\mathrm{g} / \mathrm{mL})$ & 1.742 & 1.295 & 1.344 \\
Vol\% of Cent. Solids & 22.0 & 27.1 & 25.0 \\
Wt\% Cent. Solids & 27.2 & 32.5 & 30.8 \\
Supernate Density $(\mathrm{g} / \mathrm{mL})$ & 1.314 & 0.998 & 1.001 \\
Wt\% Total Solids & 43.8 & 12.7 & 11.8 \\
Wt\% Oven Dried Solids & 51.9 & 38.3 & 36.2 \\
Wt\% UDS & 5.13 & 12.4 & 11.0 \\
Wt\% Dissolved Solids & 40.8 & 0.358 & 0.944 \\
Wt\% Supernate Liquid & 72.8 & 67.3 & 68.9 \\
\hline
\end{tabular}


Table 6.53. PEP Parallel Test D Physical Properties for Test 2

\begin{tabular}{lccc}
\hline \multicolumn{1}{c}{ Property } & D_LAB_CL_2_IS_SL & D_LAB_CL_2_ISW1_SL & D_LAB_CL_2_ISW2_SL \\
\hline Sample Description & Initial Slurry & Initial Washed Slurry-1 & Initial Washed Slurry-2 \\
\hline Bulk Density $(\mathrm{g} / \mathrm{mL})$ & 1.425 & 1.101 & 1.130 \\
Density of Cent. Solids (g/mL) & 1.697 & 1.559 & 1.379 \\
Vol\% of Cent. Solids & 20.4 & 20.4 & 28.6 \\
Wt\% Cent. Solids & 24.3 & 28.9 & 34.9 \\
Supernate Density (g/mL) & 1.355 & 0.979 & 1.023 \\
Wt\% Total Solids & 41.6 & 12.9 & 14.6 \\
Wt\% Oven Dried Solids & 51.8 & 44.8 & 41.8 \\
Wt\% UDS & 5.30 & 13.0 & 14.6 \\
Wt\% Dissolved Solids & 38.3 & 0 & 0.025 \\
Wt\% Supernate Liquid & 75.7 & 70.8 & 64.6 \\
\hline
\end{tabular}

Table 6.53 (cont.)

\begin{tabular}{lccc}
\hline \multicolumn{1}{c}{ Property } & D_LAB_CL_2_FS1_SL & D_LAB_CL_2_FSW1_SL & D_LAB_CL_2_FSW2_SL \\
\hline Sample Description & Final Slurry & Final Washed Slurry-1 & Final Washed Slurry-2 \\
\hline Bulk Density $(\mathrm{g} / \mathrm{mL})$ & 1.442 & 1.110 & 1.102 \\
Density of Cent. Solids $(\mathrm{g} / \mathrm{mL})$ & 1.863 & 1.392 & 1.305 \\
Vol\% of Cent. Solids & 18.4 & 25.5 & 27.9 \\
Wt\% Cent. Solids & 23.7 & 32.0 & 33.0 \\
Supernate Density $(\mathrm{g} / \mathrm{mL})$ & 1.347 & 1.009 & 1.021 \\
Wt $\%$ Total Solids & 42.5 & 12.2 & 12.9 \\
Wt $\%$ Oven Dried Solids & 50.8 & 37.2 & 37.4 \\
Wt $\%$ UDS & 4.27 & 11.8 & 12.2 \\
Wt $\%$ Dissolved Solids & 40.0 & 0.385 & 0.809 \\
Wt $\%$ Supernate Liquid & 76.2 & 67.7 & 66.8 \\
\hline
\end{tabular}

\subsection{PEP Tests A, B, and D Oxidative Leach Parallel Laboratory Tests}

For wastes that have significantly high chromium content, the caustic leaching and slurry dewatering are followed by adding sodium permanganate to UFP-VSL-T02A, and the slurry is subjected to oxidative leaching at nominally ambient temperature. The purpose of the oxidative leaching is to selectively oxidize the poorly alkaline-soluble Cr(III) believed to be the insoluble form in Hanford tank sludge to the much more alkaline-soluble $\mathrm{Cr}(\mathrm{VI})$, e.g., chromate.

The objectives of these tests were to provide data to support the development of scale factors between laboratory process measurements and those of PEP related to oxidative leaching. The testing included the following tests with simulated Hanford tank waste:

- Integrated Test A: Demonstrated integrated processing when caustic leaching $\left(98^{\circ} \mathrm{C}\right)$ is performed in UFP-VSL-00001A/B (PEP equivalent: UFP-VSL-T01A\&B) with the $\mathrm{Cr}$ simulant component added after the post-caustic-leach washing step. 
- Integrated Test B: Demonstrated integrated processing when the caustic leaching $\left(98^{\circ} \mathrm{C}\right)$ was performed in UFP-VSL-00002A (PEP equivalent: UFP-VSL-T02A) with the Cr simulant component added after the post-caustic-leach washing step.

- Integrated Test D: Demonstrated integrated processing when the caustic leaching is performed at a lower temperature $\left(85^{\circ} \mathrm{C}\right)$ in UFP-VSL-00002A (PEP equivalent: UFP-VSL-T02A) and with the $\mathrm{Cr}$ simulant component added to the initial batch of simulant.

Following the post-caustic-leach wash, the oxidative leach process in PEP Tests A and B proceeded according to TI-065 and TI-066, respectively. First, chromium oxyhydroxide slurry was added in-line. The slurry was then dewatered, and the slurry was washed to reduce the $\mathrm{OH}^{-}$to a targeted concentration of $\approx 0.25 \mathrm{M}$. During these wash steps, AFA was added periodically. Following completion of the Cr-containing slurry washing, a sample of slurry was taken for use in the laboratory-scale oxidative leach tests (A_LAB_OL_1 and B_LAB_OL_2).

PEP Test D was performed slightly differently in that the CrOOH component was an integral part of the simulant instead of being added after the post-caustic-leach wash, according to TI-082. Therefore, there was not an extra dewater and wash step after the CrOOH slurry was added. For PEP Test D, the laboratory-scale oxidative leach test slurry sample was removed at the conclusion of the post-caustic-leach wash step (D_LAB_OL_3 and D_LAB_OL_4).

The two PEP Test D laboratory-scale tests were performed with different amounts of permanganate as PEP had inadvertently used an excess of permanganate in their testing. Therefore, one test was performed with the amount of permanganate that PEP had used in excess (D_LAB_OL_3), and one was performed using the required amount of permanganate based on the expected quantity of $\mathrm{Cr}$ in the washed slurry based on target removal of $\mathrm{Cr}$ during caustic leach (D_LAB_OL_4). PEP had inadvertently added excess permanganate to the vessel in performing the oxidative leach of Test $\mathrm{D}$. The amounts of components used in these tests are shown in Table 6.54 .

Table 6.54. PEP Parallel Oxidative Test Matrix

\begin{tabular}{llcc}
\hline \multicolumn{1}{c}{ PEP Sample ID } & Test ID & PEP Sample $(\mathrm{g})$ & $1 \mathrm{M} \mathrm{NaMnO}$ (g) \\
\hline A_AOL_018_XX_2383_RHE_4 & A_LAB_OL_1 & 707.13 & 92.85 \\
B_02_AOL_023_XX_4297_CUF_4 & B_LAB_OL_2 & 704.34 & 95.69 \\
D_AOL_017_XX_5517_CUF_4 & D_LAB_OL_3 & 753.42 & 46.60 \\
D_AOL_017_XX_5517_CUF_4 & D_LAB_OL_4 & 766.43 & 33.61 \\
\hline
\end{tabular}

These tests were performed by placing the required amount of PEP simulant and sodium permanganate in the test vessel, attaching the lid, and stirring. The time zero $(0 \mathrm{hr})$ sample was taken, and the test continued for 8 hours at $25 \pm 2{ }^{\circ} \mathrm{C}$. Samples were taken at $1,2,3,4,5,6,7$, and 8 hours and filtered immediately through a $0.45-\mu \mathrm{m}$ syringe filter. These samples were then analyzed by ICP-AES for $\mathrm{Al}, \mathrm{Cr}$, and $\mathrm{Na}$ concentration at SWRI, and the results are shown in Table 6.55 through Table 6.57. The samples were also analyzed by IC for anion concentrations, which are shown in Table 6.58 through Table 6.61. Slurry samples were also taken at the beginning and end of the test. Some slurry samples were washed three times with $0.01 \mathrm{M} \mathrm{NaOH}$ and others were not washed. These results are shown in Table 6.62 through Table 6.65. The density of the supernate and the slurry was measured and is shown in 
Table 6.66 and Table 6.67, respectively. Table 6.68 through Table 6.71 show the measurements of the UDS in the slurries (both washed and unwashed).

Table 6.55. Supernate Al Analysis from PEP Parallel Test Oxidative Leach

\begin{tabular}{ccccc}
\hline $\begin{array}{c}\text { Time } \\
(\mathrm{hrs})\end{array}$ & $\begin{array}{c}\text { A_LAB_OL_1 } \\
(\mathrm{mg} \mathrm{Al} / \mathrm{kg} \\
\text { soln })\end{array}$ & $\begin{array}{c}\text { B_LAB_OL_1 } \\
(\mathrm{mg} \mathrm{Al} / \mathrm{kg} \text { soln })\end{array}$ & $\begin{array}{c}\text { D_LAB_OL_3 } \\
(\mathrm{mg} \mathrm{A1} / \mathrm{kg} \text { soln })\end{array}$ & $\begin{array}{c}\text { D_LAB_OL_4 } \\
(\mathrm{mg} \mathrm{Al} / \mathrm{kg} \\
\text { soln })\end{array}$ \\
\hline Initial & 330 & 247 & 412 & 419 \\
0 & 170 & 117 & 387 & 369 \\
1 & 183 & 135 & 347 & 398 \\
2 & 186 & 141 & 322 & 346 \\
3 & 182 & 146 & 303 & 340 \\
4 & 186 & 149 & 303 & 342 \\
5 & 187 & 145 & 305 & 341 \\
6 & 187 & 143 & 293 & 333 \\
7 & 188 & 143 & 297 & 336 \\
8 & 184 & 147 & 293 & 334 \\
\hline
\end{tabular}

Table 6.56. Supernate Cr Analysis from PEP Parallel Test Oxidative Leach

\begin{tabular}{ccccc}
\hline $\begin{array}{c}\text { Time } \\
(\mathrm{hrs})\end{array}$ & $\begin{array}{c}\text { A_LAB_OL_1 } \\
(\mathrm{mg} \mathrm{Cr} / \mathrm{kg} \\
\text { soln })\end{array}$ & $\begin{array}{c}\text { B_LAB_OL_1 } \\
(\mathrm{mg} \mathrm{Cr} / \mathrm{kg} \text { soln })\end{array}$ & $\begin{array}{c}\text { D_LAB_OL_3 } \\
(\mathrm{mg} \mathrm{Cr} / \mathrm{kg} \text { soln })\end{array}$ & $\begin{array}{c}\text { D_LAB_OL_4 } \\
(\mathrm{mg} \mathrm{Cr} / \mathrm{kg} \\
\text { soln })\end{array}$ \\
\hline Initial & 323 & 172 & 44.4 & 44.9 \\
0 & 6,630 & 6,880 & 116 & 116 \\
1 & 6,770 & 6,800 & 123 & 132 \\
2 & 6,800 & 6,760 & 131 & 132 \\
3 & 6,770 & 6,920 & 132 & 136 \\
4 & 6,880 & 7,010 & 138 & 143 \\
5 & 6,940 & 6,870 & 143 & 143 \\
6 & 6,870 & 6,890 & 139 & 143 \\
7 & 6,840 & 6,870 & 142 & 145 \\
8 & 6,850 & 7,080 & 142 & 146 \\
\hline
\end{tabular}


Table 6.57. Supernate Na Analysis from PEP Parallel Test Oxidative Leach

\begin{tabular}{ccccc}
\hline $\begin{array}{c}\text { Time } \\
\text { (hrs })\end{array}$ & $\begin{array}{c}\text { A_LAB_OL_1 } \\
(\mathrm{mg} \mathrm{Na} / \mathrm{kg} \text { soln })\end{array}$ & $\begin{array}{c}\text { B_LAB_OL_1 } \\
(\mathrm{mg} \mathrm{Na} / \mathrm{kg} \text { soln })\end{array}$ & $\begin{array}{c}\text { D_LAB_OL_3 } \\
(\mathrm{mg} \mathrm{Na} / \mathrm{kg} \text { soln })\end{array}$ & $\begin{array}{c}\text { D_LAB_OL_4 } \\
(\mathrm{mg} \text { Na/kg soln })\end{array}$ \\
\hline Initial & 8,220 & 8,890 & 7,440 & 7,130 \\
0 & 8,750 & 9,390 & 8,150 & 7,840 \\
1 & 9,170 & 9,410 & 8,200 & 8,350 \\
2 & 9,110 & 9,430 & 8,060 & 7,760 \\
3 & 8,820 & 9,550 & 7,710 & 7,550 \\
4 & 9,080 & 9,820 & 7,920 & 7,310 \\
5 & 8,960 & 9,450 & 8,130 & 7,600 \\
6 & 9,080 & 9,690 & 7,690 & 7,450 \\
7 & 9,270 & 9,600 & 7,720 & 7,670 \\
8 & 8,900 & 9,830 & 7,790 & 7,670 \\
\hline
\end{tabular}

Table 6.58. Anion Analysis of A_LAB_OL_1 Supernate from Oxidative Leach Tests

\begin{tabular}{ccccccc}
\hline Time (hrs) & $\begin{array}{c}\text { Chloride } \\
(\mathrm{mg} / \mathrm{kg} \\
\text { soln })\end{array}$ & $\begin{array}{c}\text { Nitrate } \\
(\mathrm{mg} / \mathrm{kg} \\
\text { soln })\end{array}$ & $\begin{array}{c}\text { Nitrite } \\
(\mathrm{mg} / \mathrm{kg} \\
\text { soln })\end{array}$ & $\begin{array}{c}\text { Oxalate } \\
(\mathrm{mg} / \mathrm{kg} \\
\text { soln })\end{array}$ & $\begin{array}{c}\text { Phosphate } \\
(\mathrm{mg} / \mathrm{kg} \text { soln })\end{array}$ & $\begin{array}{c}\text { Sulfate } \\
(\mathrm{mg} / \mathrm{kg} \\
\text { soln })\end{array}$ \\
\hline Initial & 164 & 3,790 & 70.6 & 674 & 275 & 38.4 \\
0 & 135 & 3,120 & 61.1 & 552 & 172 & 132 \\
1 & 133 & 3,190 & 58.5 & 547 & 154 & 132 \\
2 & 137 & 3,150 & 59.5 & 549 & 161 & 137 \\
3 & 134 & 3,140 & 59.5 & 547 & 150 & 134 \\
4 & 136 & 3,210 & 57.8 & 562 & 150 & 136 \\
5 & 137 & 3,140 & 61.4 & 555 & 143 & 137 \\
6 & 136 & 3,170 & 56.8 & 552 & 149 & 136 \\
7 & 131 & 3,110 & 59.8 & 546 & 143 & 135 \\
8 & 134 & 3,100 & 61.8 & 555 & 150 & 132 \\
\hline
\end{tabular}

Table 6.59. Anion Analysis of B_LAB_OL_2 Supernate from Oxidative Leach Tests

\begin{tabular}{ccccccc}
\hline Time $(\mathrm{hrs})$ & $\begin{array}{c}\text { Chloride } \\
(\mathrm{mg} / \mathrm{kg} \text { soln })\end{array}$ & $\begin{array}{c}\text { Nitrate } \\
(\mathrm{mg} / \mathrm{kg} \text { soln })\end{array}$ & $\begin{array}{c}\text { Nitrite }(\mathrm{mg} / \mathrm{kg} \\
\text { soln })\end{array}$ & $\begin{array}{c}\text { Oxalate } \\
(\mathrm{mg} / \mathrm{kg} \text { soln })\end{array}$ & $\begin{array}{c}\text { Phosphate } \\
(\mathrm{mg} / \mathrm{kg} \text { soln })\end{array}$ & $\begin{array}{c}\text { Sulfate } \\
(\mathrm{mg} / \mathrm{kg} \text { soln })\end{array}$ \\
\hline Initial & 160 & 3,840 & 90.4 & 1,530 & 124 & 48.1 \\
0 & 132 & 3,140 & 77.9 & 1,270 & 70.8 & 151 \\
1 & 138 & 3,090 & 80.2 & 1,250 & 65.9 & 151 \\
2 & 137 & 3,130 & 76.9 & 1,270 & 62.8 & 152 \\
3 & 139 & 3,100 & 81.2 & 1,260 & 64.7 & 150 \\
4 & 137 & 3,180 & 76.6 & 1,280 & 61.3 & 153 \\
5 & 139 & 3,140 & 80.5 & 1,270 & 59.8 & 152 \\
6 & 137 & 3,150 & 77.5 & 1,270 & 72.3 & 152 \\
7 & 136 & 3,110 & 82.8 & 1,270 & 64.7 & 147 \\
8 & 137 & 3,120 & 78.9 & 1,260 & 68.3 & 153 \\
\hline
\end{tabular}


Table 6.60. Anion Analysis of D_LAB_OL_3 Supernate from Oxidative Leach Tests

\begin{tabular}{ccccccc}
\hline Time $(\mathrm{hrs})$ & $\begin{array}{c}\text { Chloride } \\
(\mathrm{mg} / \mathrm{kg} \text { soln })\end{array}$ & $\begin{array}{c}\text { Nitrate } \\
(\mathrm{mg} / \mathrm{kg} \text { soln })\end{array}$ & $\begin{array}{c}\text { Nitrite }(\mathrm{mg} / \mathrm{kg} \\
\text { soln })\end{array}$ & $\begin{array}{c}\text { Oxalate } \\
(\mathrm{mg} / \mathrm{kg} \text { soln })\end{array}$ & $\begin{array}{c}\text { Phosphate } \\
(\mathrm{mg} / \mathrm{kg} \text { soln })\end{array}$ & $\begin{array}{c}\text { Sulfate } \\
(\mathrm{mg} / \mathrm{kg} \text { soln })\end{array}$ \\
\hline Initial & $<17.9$ & 509 & 145 & 6,990 & 59.1 & 141 \\
0 & 14.3 & 483 & 129 & 7,980 & 60.7 & 186 \\
1 & 16.0 & 483 & 130 & 7,990 & 68.0 & 190 \\
2 & $<17.9$ & 500 & 142 & 7,980 & 74.2 & 195 \\
3 & $<16.3$ & 483 & 136 & 7,860 & 72.9 & 189 \\
4 & $<16.1$ & 487 & 134 & 8,020 & 72.6 & 190 \\
5 & $<17.6$ & 500 & 137 & 8,030 & 76.0 & 191 \\
6 & $<15.5$ & 478 & 131 & 8,050 & 69.9 & 187 \\
7 & $<18.1$ & 487 & 137 & 7,950 & 64.0 & 191 \\
8 & $<18.8$ & 492 & 140 & 8,110 & 77.8 & 187 \\
\hline
\end{tabular}

Table 6.61. Anion Analysis of D_LAB_OL_4 Supernate from Oxidative Leach Tests

\begin{tabular}{ccccccc}
\hline Time (hrs) & $\begin{array}{c}\text { Chloride } \\
(\mathrm{mg} / \mathrm{kg} \text { soln })\end{array}$ & $\begin{array}{c}\text { Nitrate } \\
(\mathrm{mg} / \mathrm{kg} \text { soln })\end{array}$ & $\begin{array}{c}\text { Nitrite }(\mathrm{mg} / \mathrm{kg} \\
\text { soln })\end{array}$ & $\begin{array}{c}\text { Oxalate } \\
(\mathrm{mg} / \mathrm{kg} \text { soln })\end{array}$ & $\begin{array}{c}\text { Phosphate } \\
(\mathrm{mg} / \mathrm{kg} \text { soln })\end{array}$ & $\begin{array}{c}\text { Sulfate } \\
(\mathrm{mg} / \mathrm{kg} \text { soln })\end{array}$ \\
\hline Initial & $<17.4$ & 523 & 152 & 7,470 & 60.4 & 156 \\
0 & 14.2 & 487 & 134 & 7,610 & 61.6 & 179 \\
1 & $<17.9$ & 514 & 143 & 8,020 & 71.1 & 185 \\
2 & $<16.6$ & 500 & 138 & 7,890 & 68.0 & 177 \\
3 & 16.0 & 492 & 135 & 7,850 & 63.1 & 179 \\
4 & $<17.3$ & 505 & 144 & 7,860 & 68.0 & 178 \\
5 & $<19.6$ & 518 & 146 & 7,860 & 70.5 & 183 \\
6 & $<19.4$ & 509 & 145 & 7,680 & 74.8 & 181 \\
7 & $<19.2$ & 514 & 144 & 7,870 & 72.0 & 182 \\
8 & $<16.3$ & 500 & 138 & 7,960 & 70.8 & 179 \\
\hline
\end{tabular}


Table 6.62. Slurry Analysis from PEP Parallel Oxidative Leach Test A (Test 1)

\begin{tabular}{|c|c|c|c|c|c|c|}
\hline & $\begin{array}{l}\text { A_LAB_ } \\
\text { OL_1_OS_SL } \\
\text { (mg/kg slurry) }\end{array}$ & $\begin{array}{c}\text { A_LAB_ } \\
\text { OL_1_OSW_SL } \\
\text { (mg/kg slurry) }\end{array}$ & $\begin{array}{c}\text { A_LAB_} \\
\text { OL_1_IS_SL } \\
\text { (mg/kg slurry) }\end{array}$ & $\begin{array}{c}\text { A_LAB_ } \\
\text { OL_1_ISW_SL } \\
\text { (mg/kg slurry) }\end{array}$ & $\begin{array}{l}\text { A_LAB_ } \\
\text { OL_1_FS_SL } \\
\text { (mg/kg slurry) }\end{array}$ & $\begin{array}{c}\text { A_LAB_ } \\
\text { OL_1_FSW_SL } \\
\text { (mg/kg slurry) }\end{array}$ \\
\hline & $\begin{array}{c}\text { Original (before } \\
\mathrm{MnO}_{4}^{2-} \text { added) } \\
\text { Slurry }\end{array}$ & $\begin{array}{l}\text { Original Washed } \\
\text { (before } \mathrm{MnO}_{4}^{2-} \\
\text { added) Slurry }\end{array}$ & $\begin{array}{c}\text { Initial (after } \\
\mathrm{MnO}_{4}{ }^{2-} \text { added) } \\
\text { Slurry }\end{array}$ & $\begin{array}{c}\text { Initial (after } \\
\mathrm{MnO}_{4}{ }^{2-} \text { added) } \\
\text { Washed Slurry }\end{array}$ & Final Slurry & $\begin{array}{c}\text { Final Washed } \\
\text { Slurry }\end{array}$ \\
\hline $\mathrm{Al}$ & 36,200 & 54,400 & 32,800 & 51,000 & 32,800 & 49,200 \\
\hline $\mathrm{Ca}$ & 1,010 & 1,380 & 905 & 1,380 & 900 & 1,320 \\
\hline $\mathrm{Cr}$ & 6,860 & 8,860 & 6,120 & 860 & 6,110 & 854 \\
\hline $\mathrm{Fe}$ & 32,700 & 41,800 & 29,200 & 44,400 & 29,200 & 42,400 \\
\hline $\mathrm{Mg}$ & 696 & 987 & 621 & 945 & 617 & 908 \\
\hline $\mathrm{Mn}$ & 6,940 & 9,810 & 11,800 & 17,800 & 11,800 & 17,100 \\
\hline $\mathrm{Ni}$ & 1,010 & 1,340 & 895 & 1,360 & 896 & 1,300 \\
\hline $\mathrm{Na}$ & 10,100 & 4,220 & 11,300 & 4,880 & 10,900 & 4,710 \\
\hline $\mathrm{P}$ & 131 & 199 & 130 & 247 & 121 & 210 \\
\hline $\mathrm{Sr}$ & 382 & 511 & 341 & 523 & 344 & 496 \\
\hline $\mathrm{Zr}$ & 466 & 714 & 402 & 833 & 382 & 703 \\
\hline
\end{tabular}

Table 6.63. Slurry Analysis from PEP Parallel Oxidative Leach Test B (Test 2)

\begin{tabular}{|c|c|c|c|c|c|c|}
\hline & $\begin{array}{c}\text { B_LAB_ } \\
\text { OL_2_OS_SL } \\
\text { (mg/kg slurry) }\end{array}$ & $\begin{array}{c}\text { B_LAB_ } \\
\text { OL_2_OSW_SL } \\
\text { (mg/kg slurry) }\end{array}$ & $\begin{array}{c}\text { B_LAB_ } \\
\text { OL_2_IS_SL } \\
\text { (mg/kg slurry) }\end{array}$ & $\begin{array}{l}\text { B_LAB_ } \\
\text { OL_2_ISW_SL } \\
\text { (mg/kg slurry) }\end{array}$ & $\begin{array}{c}\text { B_LAB_ } \\
\text { OL_2_FS_SL } \\
\text { (mg/kg slurry) }\end{array}$ & $\begin{array}{l}\text { B_LAB_ } \\
\text { OL_2_FSW_SL } \\
\text { (mg/kg slurry) }\end{array}$ \\
\hline & $\begin{array}{c}\text { Original (before } \\
\mathrm{MnO}_{4}{ }^{2-} \text { added) } \\
\text { Slurry }\end{array}$ & $\begin{array}{l}\text { Original Washed } \\
\text { (before } \mathrm{MnO}_{4}{ }^{2-} \\
\text { added) Slurry }\end{array}$ & $\begin{array}{c}\text { Initial (after } \\
\mathrm{MnO}_{4}{ }^{2-} \text { added) } \\
\text { Slurry }\end{array}$ & $\begin{array}{c}\text { Initial (after } \\
\mathrm{MnO}_{4}{ }^{2-} \text { added) } \\
\text { Washed Slurry }\end{array}$ & Final Slurry & $\begin{array}{c}\text { Final Washed } \\
\text { Slurry }\end{array}$ \\
\hline $\mathrm{Al}$ & 40,700 & 50,600 & 34,700 & 54,000 & 34,600 & 47,000 \\
\hline $\mathrm{Ca}$ & 1,150 & 1,370 & 997 & 1,410 & 1,020 & 1,260 \\
\hline $\mathrm{Cr}$ & 6,660 & 7,820 & 5,770 & 540 & 5,970 & 544 \\
\hline $\mathrm{Fe}$ & 36,600 & 44,300 & 31,900 & 46,400 & 33,100 & 41,200 \\
\hline $\mathrm{Mg}$ & 695 & 846 & 606 & 844 & 618 & 762 \\
\hline $\mathrm{Mn}$ & 7,840 & 9,580 & 12,600 & 18,100 & 12,900 & 15,900 \\
\hline $\mathrm{Ni}$ & 1,110 & 1,360 & 977 & 1,420 & 1,010 & 1,250 \\
\hline $\mathrm{Na}$ & 10,600 & 4,330 & 12,000 & 5,370 & 12,400 & 4,770 \\
\hline $\mathrm{P}$ & 149 & 188 & 150 & 171 & 148 & 179 \\
\hline $\mathrm{Sr}$ & 331 & 400 & 291 & 419 & 296 & 367 \\
\hline $\mathrm{Zr}$ & 926 & 1,180 & 841 & 1,150 & 855 & 1,100 \\
\hline
\end{tabular}


Table 6.64. Slurry Analysis from PEP Parallel Oxidative Leach Test D, Excess $\mathrm{MnO}_{4}{ }^{-}$(Test 3 )

\begin{tabular}{|c|c|c|c|c|c|c|}
\hline & $\begin{array}{l}\text { D_LAB_- } \\
\text { OL_3_OS_SL } \\
\text { (mg/kg slurry) }\end{array}$ & $\begin{array}{c}\text { D_LAB_ } \\
\text { OL_3_OSW_SL } \\
\text { (mg/kg slurry) }\end{array}$ & $\begin{array}{c}\text { D_LAB_- } \\
\text { OL_3_IS_SL } \\
\text { (mg/kg slurry) }\end{array}$ & $\begin{array}{c}\text { D_LAB_ } \\
\text { OL_3_ISW_SL } \\
\text { (mg/kg slurry) }\end{array}$ & $\begin{array}{c}\text { D_LAB_- } \\
\text { OL_3_FS_SL } \\
\text { (mg/kg slurry) }\end{array}$ & $\begin{array}{c}\text { D_LAB_ } \\
\text { OL_3_FSW_SL } \\
\text { (mg/kg slurry) }\end{array}$ \\
\hline & $\begin{array}{c}\text { Original (before } \\
\mathrm{MnO}_{4}{ }^{2-} \text { added) } \\
\text { Slurry }\end{array}$ & $\begin{array}{l}\text { Original Washed } \\
\text { (before } \mathrm{MnO}_{4}{ }^{2-} \\
\text { added) Slurry }\end{array}$ & $\begin{array}{c}\text { Initial (after } \\
\mathrm{MnO}_{4}{ }^{-2} \text { added) } \\
\text { Slurry }\end{array}$ & $\begin{array}{c}\text { Initial (after } \\
\mathrm{MnO}_{4}{ }^{2-} \text { added) } \\
\text { Washed Slurry }\end{array}$ & Final Slurry & $\begin{array}{c}\text { Final Washed } \\
\text { Slurry }\end{array}$ \\
\hline $\mathrm{Al}$ & 55,600 & 71,000 & 52,200 & 66,200 & 52,700 & 65,300 \\
\hline $\mathrm{Ca}$ & 1,070 & 1,390 & 1,010 & 1,240 & 1,010 & 1,290 \\
\hline $\mathrm{Cr}$ & 204 & 221 & 193 & 114 & 193 & 118 \\
\hline $\mathrm{Fe}$ & 34,500 & 45,700 & 32,600 & 40,500 & 32,300 & 41,600 \\
\hline $\mathrm{Mg}$ & 652 & 852 & 614 & 755 & 615 & 792 \\
\hline $\mathrm{Mn}$ & 7,560 & 10,000 & 9,700 & 11,800 & 9,850 & 12,700 \\
\hline $\mathrm{Ni}$ & 1,050 & 1,420 & 996 & 1,240 & 981 & 1,270 \\
\hline $\mathrm{Na}$ & 8,710 & 3,540 & 9,450 & 3,860 & 9,280 & 4,150 \\
\hline $\mathrm{P}$ & 88.8 & 97.4 & 84.8 & 84.3 & 83.0 & 86.7 \\
\hline $\mathrm{Sr}$ & 315 & 416 & 299 & 366 & 295 & 378 \\
\hline $\mathrm{Zr}$ & 978 & 1,260 & 926 & 1,120 & 915 & 1,150 \\
\hline
\end{tabular}

Table 6.65. Slurry Analysis from PEP Parallel Oxidative Leach Test D, Less $\mathrm{MnO}_{4}{ }^{-}$(Test 4)

\begin{tabular}{|c|c|c|c|c|c|c|}
\hline & $\begin{array}{l}\text { D_LAB_- } \\
\text { OL_4_OS_SL } \\
\text { (mg/kg slurry) }\end{array}$ & $\begin{array}{c}\text { D_LAB_ } \\
\text { OL_4_OSW_SL } \\
\text { (mg/kg slurry) }\end{array}$ & $\begin{array}{c}\text { D_LAB_- } \\
\text { OL_4_IS_SL } \\
\text { (mg/kg slurry) }\end{array}$ & $\begin{array}{l}\text { D_LAB_- } \\
\text { OL_4_ISW_SL } \\
\text { (mg/kg slurry) }\end{array}$ & $\begin{array}{c}\text { D_LAB_- } \\
\text { OL_4_FS_SL } \\
\text { (mg/kg slurry) }\end{array}$ & $\begin{array}{c}\text { D_LAB_ } \\
\text { OL_4_FSW_SL } \\
\text { (mg/kg slurry) }\end{array}$ \\
\hline & $\begin{array}{c}\text { Original (before } \\
\mathrm{MnO}_{4}{ }^{2-} \text { added) } \\
\text { Slurry }\end{array}$ & $\begin{array}{l}\text { Original Washed } \\
\text { (before } \mathrm{MnO}_{4}{ }^{2-} \\
\text { added) Slurry }\end{array}$ & $\begin{array}{c}\text { Initial (after } \\
\mathrm{MnO}_{4}{ }^{2-} \text { added) } \\
\text { Slurry }\end{array}$ & $\begin{array}{c}\text { Initial (after } \\
\mathrm{MnO}_{4}{ }^{2-} \text { added) } \\
\text { Washed Slurry }\end{array}$ & Final Slurry & $\begin{array}{l}\text { Final Washed } \\
\text { Slurry }\end{array}$ \\
\hline $\mathrm{Al}$ & 58,700 & 75,700 & 54,700 & 71,700 & 50,100 & 64,400 \\
\hline $\mathrm{Ca}$ & 1,090 & 1,390 & 1,020 & 1,330 & 1,030 & 1,250 \\
\hline $\mathrm{Cr}$ & 210 & 223 & 197 & 124 & 198 & 108 \\
\hline $\mathrm{Fe}$ & 35,700 & 45,900 & 33,200 & 44,000 & 33,300 & 40,300 \\
\hline $\mathrm{Mg}$ & 658 & 854 & 622 & 808 & 626 & 762 \\
\hline $\mathrm{Mn}$ & 7,880 & 10,200 & 9,490 & 12,300 & 9,310 & 11,200 \\
\hline $\mathrm{Ni}$ & 1,100 & 1,400 & 1,010 & 1,360 & 1,020 & 1,240 \\
\hline $\mathrm{Na}$ & 8,540 & 3,380 & 8,970 & 3,990 & 9,270 & 3,690 \\
\hline $\mathrm{P}$ & 91.7 & 103 & 89.0 & 91.2 & 87.3 & 87.1 \\
\hline $\mathrm{Sr}$ & 321 & 437 & 310 & 393 & 302 & 368 \\
\hline $\mathrm{Zr}$ & 1,000 & 1,300 & 953 & 1,240 & 943 & 1,120 \\
\hline
\end{tabular}


Table 6.66. PEP Parallel Oxidative Test Supernate Density

\begin{tabular}{lclc}
\hline \multicolumn{1}{c}{ Sample ID } & $\begin{array}{c}\text { Density } \\
(\mathrm{g} / \mathrm{mL})\end{array}$ & \multicolumn{1}{c}{ Sample ID } & $\begin{array}{c}\text { Density } \\
(\mathrm{g} / \mathrm{mL})\end{array}$ \\
\hline A_LAB_OL_1_ORG_SUP & 1.018 & D_LAB_OL_3_ORG_SUP & 1.024 \\
A_LAB_OL_1_INA_SUP & 1.044 & D_LAB_OL_3_INA_SUP & 1.029 \\
A_LAB_OL_1_001 & 1.030 & D_LAB_OL_3_001 & 1.017 \\
A_LAB_OL_1_002 & 1.045 & D_LAB_OL_3_002 & 1.030 \\
A_LAB_OL_1_003 & 1.051 & D_LAB_OL_3_003 & 1.027 \\
A_LAB_OL_1_004 & 1.036 & D_LAB_OL_3_004 & 1.024 \\
A_LAB_OL_1_005 & 1.043 & D_LAB_OL_3_005 & 1.037 \\
A_LAB_OL_1_006 & 1.046 & D_LAB_OL_3_006 & 1.027 \\
A_LAB_OL_1_007 & 1.042 & D_LAB_OL_3_007 & 1.028 \\
A_LAB_OL_1_008 & 1.042 & D_LAB_OL_3_008 & 1.034 \\
B_LAB_OL_2_ORG_SUP & 1.022 & D_LAB_OL_4_ORG_SUP & 1.016 \\
B_LAB_OL_2_INA_SUP & 1.043 & D_LAB_OL_4_INA_SUP & 1.024 \\
B_LAB_OL_2_001 & 1.046 & D_LAB_OL_4_001 & 1.031 \\
B_LAB_OL_2_002 & 1.042 & D_LAB_OL_4_002 & 1.029 \\
B_LAB_OL_2_003 & 1.043 & D_LAB_OL_44003 & 1.023 \\
B_LAB_OL_2_004 & 1.047 & D_LAB_OL_4_004 & 1.029 \\
B_LAB_OL_2_005 & 1.040 & D_LAB_OL_4_005 & 1.026 \\
B_LAB_OL_2_006 & 1.047 & D_LAB_OL_4_006 & 1.029 \\
B_LAB_OL_2_007 & 1.046 & D_LAB_OL_4_007 & 1.031 \\
B_LAB_OL_2_008 & 1.041 & D_LAB_OL_4_008 & 1.030 \\
\hline
\end{tabular}


Table 6.67. PEP Parallel Oxidative Test Slurry Density

\begin{tabular}{|c|c|c|c|c|c|}
\hline Sample ID & $\begin{array}{c}\text { Sample } \\
\text { Description }\end{array}$ & $\begin{array}{l}\text { Density } \\
(\mathrm{g} / \mathrm{mL})\end{array}$ & Sample ID & $\begin{array}{c}\text { Sample } \\
\text { Description }\end{array}$ & $\begin{array}{r}\text { Density } \\
(\mathrm{g} / \mathrm{mL})\end{array}$ \\
\hline A_LAB_OL_1_FS_SL & $\begin{array}{c}\text { Final Slurry- } \\
\text { Test } 1\end{array}$ & 1.145 & D_LAB_OL_3_FS_SL & $\begin{array}{c}\text { Final Slurry- } \\
\text { Test } 3\end{array}$ & 1.156 \\
\hline A_LAB_OL_1_FSW_SL & $\begin{array}{l}\text { Final Washed } \\
\text { Slurry-Test } 1\end{array}$ & 1.149 & $\begin{array}{c}\text { D_LAB_OL_3 } \\
\text { FSW_SL }\end{array}$ & $\begin{array}{c}\text { Final Washed } \\
\text { Slurry-Test } 3\end{array}$ & 1.154 \\
\hline A_LAB_OL_1_IS_SL & $\begin{array}{c}\text { Initial (after } \\
\mathrm{MnO}_{4}{ }^{2-} \text { added) } \\
\text { Slurry-Test } 1\end{array}$ & 1.148 & D_LAB_OL_3_IS_SL & $\begin{array}{c}\text { Initial (after } \\
\mathrm{MnO}_{4}{ }^{2-} \text { added) } \\
\text { Slurry-Test } 3\end{array}$ & 1.149 \\
\hline A_LAB_OL_1_ISW_SL & $\begin{array}{c}\text { Initial (after } \\
\mathrm{MnO}_{4}^{2-} \text { added) } \\
\text { Washed Slurry- } \\
\text { Test } 1\end{array}$ & 1.148 & $\begin{array}{c}\text { D_LAB_OL_3 } \\
\text { ISW_SL }\end{array}$ & $\begin{array}{c}\text { Initial (after } \\
\mathrm{MnO}_{4}^{2-} \text { added) } \\
\text { Washed Slurry- } \\
\text { Test } 3\end{array}$ & 1.143 \\
\hline A_LAB_OL_1_OS_SL & $\begin{array}{c}\text { Original } \\
\text { (before } \mathrm{MnO}_{4}{ }^{2-} \\
\text { added) Slurry- } \\
\text { Test } 1\end{array}$ & 1.143 & $\underset{\text { OS_SL }}{\text { D_LAB_OL_3 }_{-}}$ & $\begin{array}{c}\text { Original } \\
\text { (before } \mathrm{MnO}_{4}^{2-} \\
\text { added) Slurry- } \\
\text { Test } 3\end{array}$ & 1.161 \\
\hline A_LAB_OL_1_OWS_SL & $\begin{array}{c}\text { Original } \\
\text { Washed } \\
\text { (before } \mathrm{MnO}_{4}^{2-} \\
\text { added) Slurry- } \\
\text { Test } 1\end{array}$ & 1.211 & $\begin{array}{c}\text { D_LAB_OL_3 } \\
\text { OWS_SL }\end{array}$ & $\begin{array}{c}\text { Original } \\
\text { Washed (before } \\
\mathrm{MnO}_{4}{ }^{2-} \text { added) } \\
\text { Slurry-Test } 3\end{array}$ & 1.200 \\
\hline B_LAB_OL_2_FS_SL & $\begin{array}{c}\text { Final Slurry- } \\
\text { Test } 2\end{array}$ & 1.151 & D_LAB_OL_4_FS_SL & $\begin{array}{c}\text { Final Slurry- } \\
\text { Test } 4\end{array}$ & 1.159 \\
\hline B_LAB_OL_2_FSW_SL & $\begin{array}{l}\text { Final Washed } \\
\text { Slurry-Test } 2\end{array}$ & 1.141 & $\begin{array}{c}\text { D_LAB_OL_4 } \\
\text { FSW_SL }\end{array}$ & $\begin{array}{l}\text { Final Washed } \\
\text { Slurry-Test } 4\end{array}$ & 1.173 \\
\hline B_LAB_OL_2_IS_SL & $\begin{array}{c}\text { Initial (after } \\
\mathrm{MnO}_{4}{ }^{2-} \text { added) } \\
\text { Slurry-Test } 2\end{array}$ & 1.153 & D_LAB_OL_4_IS_SL & $\begin{array}{c}\text { Initial (after } \\
\mathrm{MnO}_{4}{ }^{2-} \text { added) } \\
\text { Slurry-Test } 4\end{array}$ & 1.141 \\
\hline B_LAB_OL_2_ISW_SL & $\begin{array}{c}\text { Initial (after } \\
\mathrm{MnO}_{4}^{2-} \text { added) } \\
\text { Washed Slurry- } \\
\text { Test } 2\end{array}$ & 1.155 & $\begin{array}{c}\text { D_LAB_OL_4_- } \\
\text { ISW_SL }\end{array}$ & $\begin{array}{c}\text { Initial (after } \\
\mathrm{MnO}_{4}^{2-} \text { added) } \\
\text { Washed Slurry- } \\
\text { Test } 4\end{array}$ & 1.180 \\
\hline B_LAB_OL_2_OS_SL & $\begin{array}{c}\text { Original } \\
\text { (before } \mathrm{MnO}_{4}^{2-} \\
\text { added) Slurry- } \\
\text { Test } 2\end{array}$ & 1.149 & $\underset{\text { OS_SL }}{\text { D_LAB_OL_4 }}$ & $\begin{array}{c}\text { Original } \\
\text { (before } \mathrm{MnO}_{4}^{2-} \\
\text { added) Slurry- } \\
\text { Test } 4\end{array}$ & 1.176 \\
\hline B_LAB_OL_2_OWS_SL & $\begin{array}{c}\text { Original } \\
\text { Washed } \\
\text { (before } \mathrm{MnO}_{4}{ }^{2-} \\
\text { added) Slurry- } \\
\text { Test } 2\end{array}$ & 1.166 & $\begin{array}{c}\text { D_LAB_OL_4 } \\
\text { OWS_SL }\end{array}$ & $\begin{array}{c}\text { Original } \\
\text { Washed (before } \\
\mathrm{MnO}_{4}{ }^{2-} \text { added) } \\
\text { Slurry-Test } 4\end{array}$ & 1.224 \\
\hline
\end{tabular}


Table 6.68. PEP Parallel Oxidative Test A_LAB_OL_1 Physical Properties

\begin{tabular}{|c|c|c|c|c|c|c|}
\hline \multirow[b]{2}{*}{ Property } & \multicolumn{6}{|c|}{ A LAB OL 1} \\
\hline & $\overline{\text { FS_SL }}$ & $\overline{\text { FSW_SL }}$ & IS_SL & ISW_SL & OS_SL & OWS_SL \\
\hline Sample Description & $\begin{array}{l}\text { Final } \\
\text { Slurry }\end{array}$ & $\begin{array}{c}\text { Final } \\
\text { Washed } \\
\text { Slurry }\end{array}$ & $\begin{array}{c}\text { Initial } \\
\text { (after } \\
\mathrm{MnO}_{4}{ }^{2-} \\
\text { added) } \\
\text { Slurry }\end{array}$ & 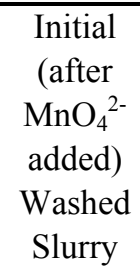 & $\begin{array}{l}\begin{array}{l}\text { Original } \\
\text { (before }\end{array} \\
\mathrm{MnO}_{4}{ }^{2-} \\
\text { added) } \\
\text { Slurry }\end{array}$ & $\begin{array}{l}\text { Original } \\
\text { Washed } \\
\text { (before } \\
\mathrm{MnO}_{4}^{2-} \\
\text { added) } \\
\text { Slurry }\end{array}$ \\
\hline Bulk Density (g/mL) & 1.170 & 1.247 & 1.194 & 1.205 & 1.165 & 1.216 \\
\hline Density of Cent. Solids ( $\mathrm{g} / \mathrm{mL}$ ) & 1.529 & 1.638 & 1.765 & 1.423 & 1.425 & 1.423 \\
\hline Vol\% of Cent. Solids & 30.4 & 41.5 & 27.3 & 46.5 & 36.4 & 46.9 \\
\hline $\mathrm{Wt} \%$ Cent. Solids & 39.8 & 54.5 & 40.3 & 55.0 & 44.5 & 54.9 \\
\hline Supernate Density $(\mathrm{g} / \mathrm{mL})$ & 1.008 & 0.955 & 0.968 & 1.001 & 1.005 & 1.019 \\
\hline $\mathrm{Wt} \%$ Total Solids & 19.3 & 24.6 & 19.3 & 25.3 & 20.4 & 25.8 \\
\hline $\mathrm{Wt} \%$ Oven Dried Solids & 43.6 & 45.0 & 42.9 & 45.8 & 43.1 & 46.7 \\
\hline $\mathrm{Wt} \%$ UDS & 16.6 & 24.5 & 16.5 & 25.1 & 18.6 & 25.6 \\
\hline Wt\% Dissolved Solids & 3.30 & 0.236 & 3.33 & 0.365 & 2.23 & 0.302 \\
\hline $\mathrm{Wt} \%$ Supernate Liquid & 59.9 & 44.8 & 59.0 & 44.4 & 54.9 & 44.5 \\
\hline
\end{tabular}

Table 6.69. PEP Parallel Oxidative Test B LAB_OL_2 Physical Properties

\begin{tabular}{|c|c|c|c|c|c|c|}
\hline \multirow[b]{2}{*}{ Property } & \multicolumn{6}{|c|}{ B_LAB_OL_2 } \\
\hline & FS_SL & FSW_SL & IS_SL & ISW_SL & OS_SL & OWS_SL \\
\hline Sample Description & $\begin{array}{l}\text { Final } \\
\text { Slurry }\end{array}$ & $\begin{array}{c}\text { Final } \\
\text { Washed } \\
\text { Slurry }\end{array}$ & $\begin{array}{c}\text { Initial } \\
\text { (after } \\
\mathrm{MnO}_{4}{ }^{2-} \\
\text { added) } \\
\text { Slurry }\end{array}$ & $\begin{array}{c}\text { Initial } \\
\text { (after } \\
\mathrm{MnO}_{4}^{2-} \\
\text { added) } \\
\text { Washed } \\
\text { Slurry }\end{array}$ & $\begin{array}{l}\text { Original } \\
\text { (before } \\
\mathrm{MnO}_{4}{ }^{2-} \\
\text { added) } \\
\text { Slurry }\end{array}$ & $\begin{array}{l}\text { Original } \\
\text { Washed } \\
\text { (before } \\
\mathrm{MnO}_{4}{ }^{2-} \\
\text { added) } \\
\text { Slurry }\end{array}$ \\
\hline Bulk Density $(\mathrm{g} / \mathrm{mL})$ & 1.208 & 1.226 & 1.186 & 1.255 & 1.255 & 1.184 \\
\hline Density of Cent. Solids $(\mathrm{g} / \mathrm{mL})$ & 1.500 & 1.482 & 1.460 & 1.635 & 1.418 & 1.357 \\
\hline Vol\% of Cent. Solids & 36.4 & 42.9 & 36.4 & 44.1 & 43.6 & 50.0 \\
\hline Wt $\%$ Cent. Solids & 45.2 & 51.8 & 44.8 & 57.5 & 49.3 & 57.3 \\
\hline Supernate Density $(\mathrm{g} / \mathrm{mL})$ & 1.032 & 1.030 & 1.024 & 0.942 & 1.117 & 0.995 \\
\hline $\mathrm{Wt} \%$ Total Solids & 21.0 & 23.0 & 20.9 & 26.1 & 22.5 & 25.2 \\
\hline Wt $\%$ Oven Dried Solids & 42.4 & 44.1 & 42.5 & 45.2 & 43.1 & 43.9 \\
\hline $\mathrm{Wt} \%$ UDS & 18.2 & 22.8 & 18.1 & 25.9 & 20.5 & 25.1 \\
\hline Wt\% Dissolved Solids & 3.52 & 0.263 & 3.46 & 0.268 & 2.52 & 0.205 \\
\hline $\mathrm{Wt} \%$ Supernate Liquid & 54.3 & 48.0 & 54.9 & 42.0 & 50.2 & 42.0 \\
\hline
\end{tabular}


Table 6.70. PEP Parallel Test D LAB_OL_3 Physical Properties

\begin{tabular}{|c|c|c|c|c|c|c|}
\hline \multirow[b]{2}{*}{ Property } & \multicolumn{6}{|c|}{ D_LAB_OL_3 } \\
\hline & FS_SL & $\overline{\text { FSW_SL }}$ & IS_SL & ISW_SL & OS_SL & OWS_SL \\
\hline Sample Description & $\begin{array}{l}\text { Final } \\
\text { Slurry }\end{array}$ & $\begin{array}{c}\text { Final } \\
\text { Washed } \\
\text { Slurry }\end{array}$ & $\begin{array}{l}\text { Initial } \\
\text { (after } \\
\mathrm{MnO}_{4}{ }^{2-} \\
\text { added) } \\
\text { Slurry }\end{array}$ & 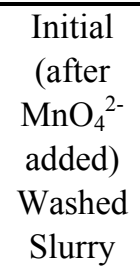 & $\begin{array}{l}\begin{array}{c}\text { Original } \\
\text { (before }\end{array} \\
\mathrm{MnO}_{4}^{2-} \\
\text { added) } \\
\text { Slurry }\end{array}$ & 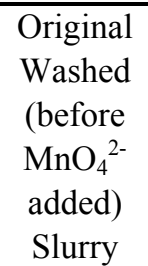 \\
\hline Bulk Density (g/mL) & 1.169 & 1.218 & 1.183 & 1.216 & 1.163 & 1.246 \\
\hline Density of Cent. Solids $(\mathrm{g} / \mathrm{mL})$ & 1.470 & 1.436 & 1.507 & 1.366 & 1.387 & 1.338 \\
\hline Vol\% of Cent. Solids & 41.0 & 51.2 & 40.0 & 52.1 & 42.5 & 56.0 \\
\hline Wt\% Cent. Solids & 51.6 & 60.4 & 51.0 & 58.5 & 50.7 & 60.1 \\
\hline Supernate Density $(\mathrm{g} / \mathrm{mL})$ & 0.940 & 0.978 & 0.954 & 1.036 & 0.986 & 1.108 \\
\hline $\mathrm{Wt} \%$ Total Solids & 23.2 & 27.5 & 23.1 & 26.6 & 23.8 & 28.5 \\
\hline $\mathrm{Wt} \%$ Oven Dried Solids & 42.8 & 45.3 & 43.2 & 45.3 & 44.8 & 47.3 \\
\hline $\mathrm{Wt} \%$ UDS & 21.3 & 27.2 & 21.3 & 26.3 & 22.1 & 28.4 \\
\hline Wt $\%$ Dissolved Solids & 2.40 & 0.476 & 2.34 & 0.361 & 2.18 & 0.160 \\
\hline $\mathrm{Wt} \%$ Supernate Liquid & 47.4 & 39.1 & 48.4 & 40.8 & 48.8 & 39.1 \\
\hline
\end{tabular}

Table 6.71. PEP Parallel Oxidative Test D LAB_OL_4 Physical Properties

\begin{tabular}{|c|c|c|c|c|c|c|}
\hline \multirow[b]{2}{*}{ Property } & \multicolumn{6}{|c|}{ D_LAB_OL_4 } \\
\hline & FS_SL & FSW_SL & IS_SL & ISW_SL & OS_SL & OWS_SL \\
\hline Sample Description & $\begin{array}{l}\text { Final } \\
\text { Slurry }\end{array}$ & $\begin{array}{c}\text { Final } \\
\text { Washed } \\
\text { Slurry }\end{array}$ & $\begin{array}{l}\text { Initial } \\
\text { (after } \\
\mathrm{MnO}_{4}{ }^{2-} \\
\text { added) } \\
\text { Slurry }\end{array}$ & 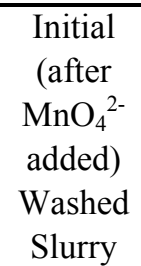 & $\begin{array}{c}\text { Original } \\
\text { (before } \\
\mathrm{MnO}_{4}{ }^{2-} \\
\text { added) } \\
\text { Slurry }\end{array}$ & $\begin{array}{c}\text { Original } \\
\text { Washed } \\
\text { (before } \\
\mathrm{MnO}_{4}{ }^{2-} \\
\text { added) } \\
\text { Slurry }\end{array}$ \\
\hline Bulk Density (g/mL) & 1.116 & 1.177 & 1.255 & 1.286 & 1.196 & 1.262 \\
\hline Density of Cent. Solids $(\mathrm{g} / \mathrm{mL})$ & 1.277 & 1.288 & 1.522 & 1.585 & 1.414 & 1.439 \\
\hline Vol\% of Cent. Solids & 45.0 & 52.5 & 41.7 & 50.0 & 42.1 & 52.6 \\
\hline $\mathrm{Wt} \%$ Cent. Solids & 51.5 & 57.4 & 50.5 & 61.6 & 49.8 & 60.1 \\
\hline Supernate Density $(\mathrm{g} / \mathrm{mL})$ & 0.974 & 1.017 & 1.055 & 0.968 & 1.025 & 1.047 \\
\hline $\mathrm{Wt} \%$ Total Solids & 23.4 & 26.0 & 23.3 & 28.2 & 23.9 & 28.3 \\
\hline Wt\% Oven Dried Solids & 43.2 & 45.1 & 43.9 & 45.5 & 45.8 & 46.9 \\
\hline $\mathrm{Wt} \%$ UDS & 21.6 & 25.8 & 21.5 & 27.9 & 22.2 & 28.1 \\
\hline $\mathrm{Wt} \%$ Dissolved Solids & 2.27 & 0.197 & 2.31 & 0.408 & 2.18 & 0.159 \\
\hline $\mathrm{Wt} \%$ Supernate Liquid & 48.0 & 41.0 & 49.0 & 37.6 & 49.6 & 39.3 \\
\hline
\end{tabular}





\subsection{Precipitation Study Results}

Significant post-filter precipitation was observed in the post-caustic-leachate and wash solutions while the leaching and ultrafiltration process was being demonstrated in the PEP. Precipitation in this stream could lead to accumulation of precipitates in filtrate receipt vessels and in the feed to the ion exchange column. The consequences of post-filtration precipitation in the feeds to the ion-exchange column will have a very significant effect on the operability of the unit and the pretreatment process. Therefore, it is essential to develop an understanding of the post-filtration precipitation phenomenon.

This section contains the results of several different tests and observations that were performed on these samples, including:

- Post-filtration precipitation of Test A solids in the wash solutions

- Blending of Test A washes and blending of Test A washes with the Test A leachate

- Observations of Test A solids in the leachate before blending

- Observations of Test B and Test D solids in the initial concentrations without blending

- Analysis by polarized light microscopy and PSD of Test D leachate precipitation and wash postfiltration precipitation.

In PEP Test A, feed stored in HLP-VSL-T22 with simulant at 5.5-wt\% UDS was transferred to either fill vessel UFP-VSL-T01A or vessel UFP-VSL-T01B in six separate batches. To each simulant batch, $19 \mathrm{M} \mathrm{NaOH}$ (nominal concentration) was added, and caustic leaching was carried out by using steam sparging to raise the temperature to $98^{\circ} \mathrm{C}$ for $16 \mathrm{hr}$, following which the partially cooled leached slurry from all six batches was transferred to UFP-VSL-T02A for solids concentration. Caustic leaching, cooling, and transferring to UFP-VSL-T02A alternated between vessel UFP-VSL-T01A and vessel UFP-VSL-T01B for a total of six batches. During caustic leaching, AFA was added to maintain a target concentration of $350 \mathrm{ppm}$ before starting the post-caustic-leach wash. Solids concentration continued until UFP-VSL-T02A was filled with leached slurry concentrated to 18.3-wt\% UDS for the washing test. The oxalate was present in excess in the solids, and both the oxalate and phosphate were present at the solubility limits in the supernate. Compared to the actual waste, the oxalate was high, but not out of the range possible for the WTP during pretreatment, and the phosphate was in the range that the WTP pretreatment may encounter (and even lower than some) because no phosphate was present in the simulant solids whereas it can be in the actual waste.

The concentrated caustic-leached solids in UFP-VSL-T02A were washed incrementally with $0.01 \mathrm{M}$ $\mathrm{NaOH}$. The wash liquid was added in steps, 11 gallons target volume for each step, 100 steps total. The wash liquid was injected in-line to the filter loop while the pumps ran and permeate was extracted by the filters. During every third wash step, AFA was added to maintain a target concentration of $350 \mathrm{ppm}$. Wash liquid additions were initiated when the level in vessel UFP-VSL-T02A dropped below a set value, occurring approximately every 4 minutes, except between batches 7 and 8 . Between batches 7 and 8 , there was a 14-hr hold time to address recirculation pump problems. During the solids washing, permeate was continuously removed at a rate between 4 and $17 \mathrm{~kg} / \mathrm{min}$ by ultrafiltration through all five filter bundles. In each wash step, permeate was sampled in 1-L bottles and then transferred to the Applied Process Engineering Laboratory (APEL) for further testing. 
The objective of the tests described here was to evaluate the propensity of the permeates from the PEP post-caustic-leach wash solutions to precipitate solids when blended in various ratios as well as when blended with leachate. The following PEP permeate blending scenarios were selected to cover the spectrum of potential permeate blending scenarios as well as to maximize the aluminate and oxalate solubility ranges. Only the samples from PEP Test A were blended and then evaluated for precipitates based on the results obtained in this testing and due to BNI's direction per e-mail confirmation on July 1, 2009, following up verbal instruction. ${ }^{\text {(a) }}$ Only Test D polarized light microscopy and particle size analysis were performed during this work. Additional analysis of Test D and Test B are described in WTP-RPT-205. ${ }^{(b)}$

All of the odd-numbered wash-step permeate samples were stored at room temperature $\left(\sim 18\right.$ to $\left.22^{\circ} \mathrm{C}\right)$ for 10 days and then examined for precipitates. A large majority (38 out of 50) of them contained significant precipitates as shown in Table 7.1. The precipitates from sample bottles \#2341 (wash step \#3) and \#2367 (wash step \#29) were examined by X-ray diffraction (XRD) to determine their composition as shown in Figure 7.1 and Figure 7.2. These showed that the majority of the precipitate is composed of oxalate and phosphate and not aluminum.

Table 7.1. PEP Test A Wash Permeate Samples

\begin{tabular}{|c|c|c|c|}
\hline Sample ID & $\begin{array}{c}\text { Wash } \\
\text { Step \# }\end{array}$ & Solids & Solids Description \\
\hline 2339 & 1 & 3 & Fine white powder type crystal and phosphate-type rods mixed \\
\hline 2341 & 3 & 3 & Fine white powder type crystal \\
\hline 2343 & 5 & 3 & Fine white powder type crystal \\
\hline 2345 & 7 & 3 & Phosphate-type rods only \\
\hline 2347 & 9 & 3 & $1 / 4$ in needle crystals perfectly uniform rods, no other crystal shapes \\
\hline 2349 & 11 & 3 & Phosphate-type rods only \\
\hline 2351 & 13 & 3 & Phosphate-type rods only \\
\hline 2353 & 15 & 3 & Phosphate-type rods only \\
\hline 2355 & 17 & 4 & Crystals attached to bottom of container in solid puck \\
\hline 2357 & 19 & 4 & Crystals attached to bottom of container in solid puck \\
\hline 2359 & 21 & 4 & Crystals attached to bottom of container in solid puck \\
\hline 2361 & 23 & 4 & Crystals attached to bottom of container in solid puck \\
\hline 2363 & 25 & 5 & Crystals attached to bottom of container in solid puck \\
\hline 2365 & 27 & 5 & Crystals attached to bottom of container in solid puck \\
\hline 2367 & 29 & 5 & Crystals attached to bottom of container in solid puck \\
\hline 2369 & 31 & 4 & Large diameter rectangular, clear crystals \\
\hline 2371 & 33 & 3 & $1 / 4$-in. needle crystals non-attached to bottle \\
\hline 2373 & 35 & 3 & $1 / 4$-in. needle crystals non-attached to bottle \\
\hline 2375 & 37 & 3 & $1 / 4$-in. needle crystals non-attached to bottle \\
\hline 2557 & 40 & 4 & $1 / 4$-in. needle crystals non-attached to bottle \\
\hline 2559 & 42 & 4 & $1 / 4$-in. needle crystals \\
\hline 2561 & 44 & 4 & $1 / 4$-in. needle crystals \\
\hline 2563 & 46 & 4 & $1 / 4$-in. needle crystals \\
\hline 2565 & 48 & 4 & $1 / 4$-in. needle crystals \\
\hline
\end{tabular}

(a) E-mail titled "E-mail Request" from Parameshwaran S Sundar on July 1, 2009 to Reid Peterson.

(b) RL Russell, RA Peterson, DE Rinehart, and JV Crum. 2009. Results of the Laboratory Precipitate Study from PEP Simulant. WTP-RPT-205, Rev. A, Bechtel National Inc., Richland, Washington. 
Table 7.1. PEP Test A Wash Permeate Samples

\begin{tabular}{|c|c|c|c|}
\hline Sample ID & $\begin{array}{l}\text { Wash } \\
\text { Step \# }\end{array}$ & Solids & Solids Description \\
\hline 2567 & 50 & 4 & 1/4-in. needle crystals \\
\hline 2569 & 52 & 4 & $1 / 4$-in. needle crystals \\
\hline 2571 & 54 & 4 & $1 / 4$-in. needle crystals \\
\hline 2573 & 56 & 4 & $1 / 4$-in. needle crystals \\
\hline 2575 & 58 & 4 & $1 / 4$-in. needle crystals \\
\hline 2577 & 60 & 4 & $1 / 4$-in. needle crystals \\
\hline 2579 & 62 & 4 & 1/4-in. needle crystals \\
\hline 2581 & 64 & 4 & $1 / 4$-in. needle crystals \\
\hline 2583 & 66 & 4 & 1/4-in. needle crystals \\
\hline 2585 & 68 & 4 & $1 / 4$-in. needle crystals \\
\hline 2587 & 70 & 4 & 1/4-in. needle crystals \\
\hline 2589 & 72 & 4 & $1 / 4$-in. needle crystals \\
\hline 2591 & 74 & 4 & 1/4-in. needle crystals \\
\hline 2593 & 76 & 1 & $\sim 15 \frac{1}{4} 4$-in. crystals non-attached to bottle \\
\hline 2595 & 78 & 0 & No precipitate visible \\
\hline 2597 & 80 & 0 & No precipitate visible \\
\hline 2621 & 82 & 0 & No precipitate visible \\
\hline 2623 & 84 & 0 & No precipitate visible \\
\hline 2625 & 86 & 0 & No precipitate visible \\
\hline 2627 & 88 & 0 & No precipitate visible \\
\hline 2629 & 90 & 0 & No precipitate visible \\
\hline 2631 & 92 & 0 & No precipitate visible \\
\hline 2633 & 94 & 0 & No precipitate visible \\
\hline 2635 & 96 & 0 & No precipitate visible \\
\hline 2637 & 98 & 0 & No precipitate visible \\
\hline 2639 & 100 & 0 & No precipitate visible \\
\hline
\end{tabular}




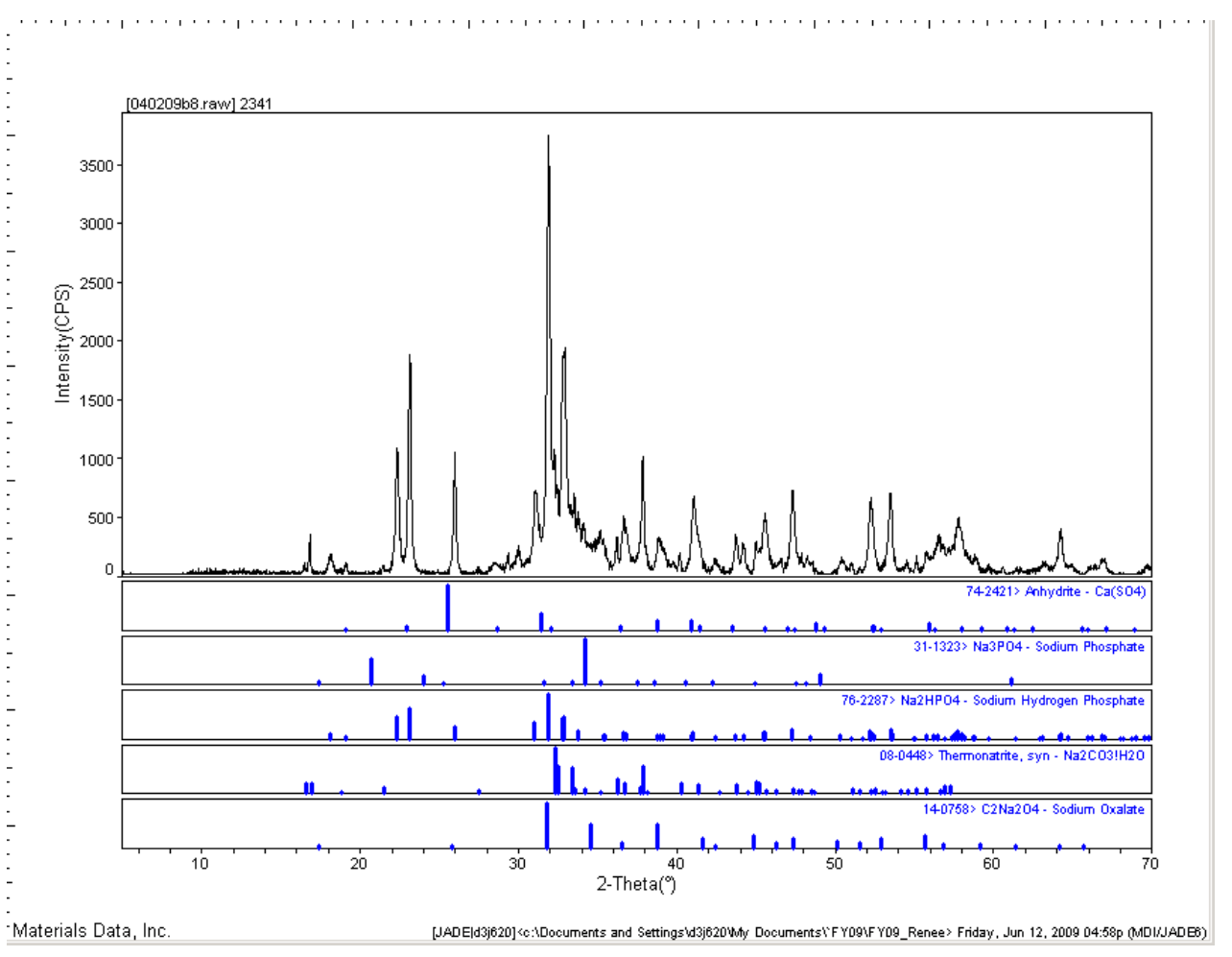

Figure 7.1. XRD Pattern of Crystals from Test A Wash Step \#3 (Sample Bottle \# 2341)

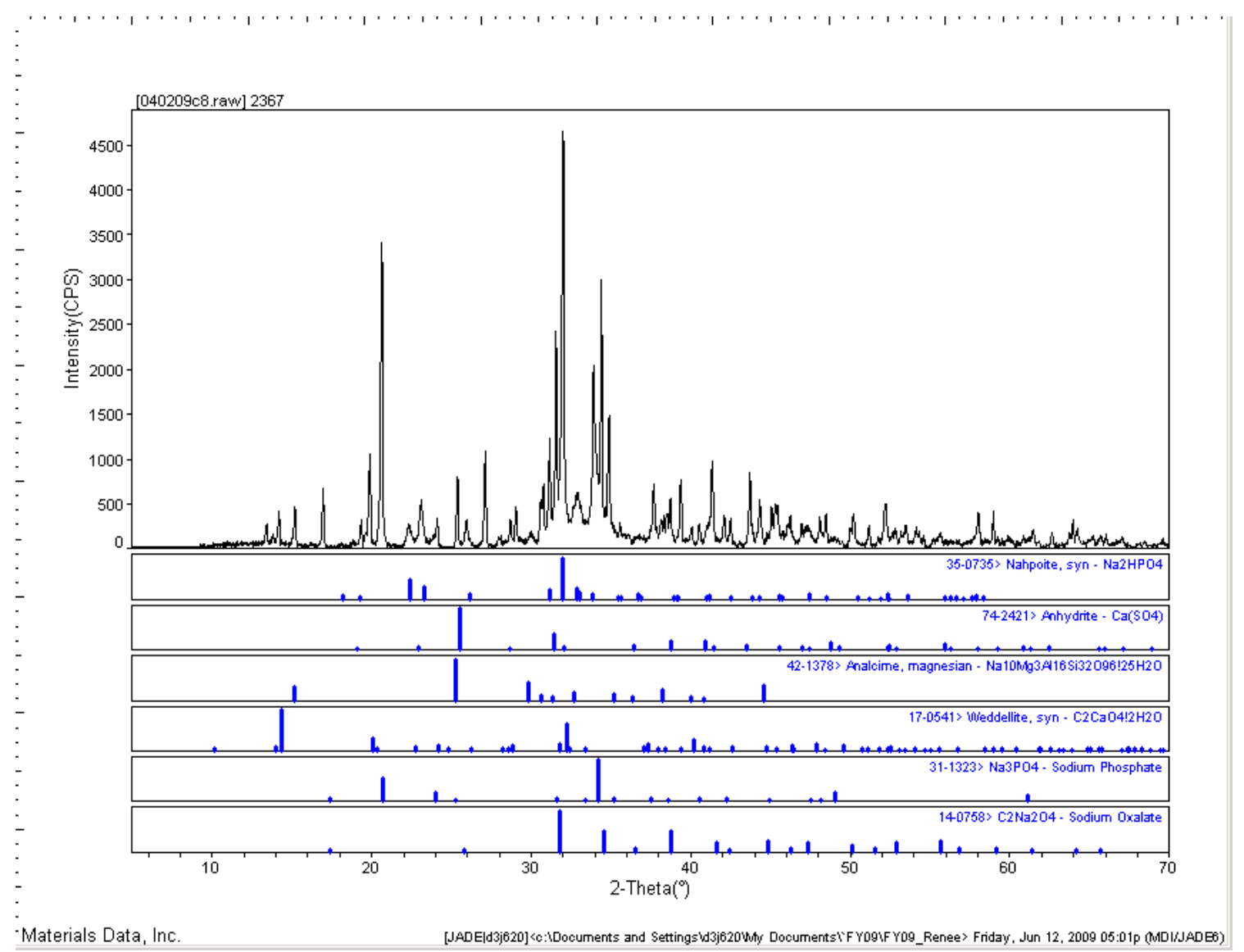


Figure 7.2. XRD Pattern of Crystals from Test A Wash \#29 (Sample Bottle \# 2367)

The solids were filtered, air-dried, and weighed for the odd-numbered wash samples with a Na concentration greater than $1.8 \mathrm{M}$. The weights are given in Table 7.2 along with the weight percent UDS present. Even after filtering, crystals continued to form in the supernate over the next month. Figure 7.3 shows the dried crystals for several of the samples.

Table 7.2. Weights of Filtered Solids in Odd-Numbered Test A Samples

\begin{tabular}{cccc}
\hline & $\begin{array}{c}\text { PEP Post-Caustic- } \\
\text { Leach Wash Step \# }\end{array}$ & $\begin{array}{c}\text { Weight of } \\
\text { Solids (g) }\end{array}$ & Wt\% UDS \\
\hline A_00PF1_015_XX_2339_ARC_4 & 1 & 0.619 & 0.048 \\
A_00PF1_015_XX_2341_ARC_4 & 3 & 1.99 & 0.17 \\
\hline A_00PF1_015_XX_2343_ARC_4 & 5 & 2.76 & 0.22 \\
A_00PF1_015_XX_2345_ARC_4 & 7 & 2.94 & 0.24 \\
A_00PF1_015_XX_2347_ARC_4 & 9 & 2.55 & 0.24 \\
A_00PF1_015_XX_2349_ARC_4 & 11 & 2.61 & 0.24 \\
A_00PF1_015_XX_2351_ARC_4 & 13 & 2.84 & 0.26 \\
A_00PF1_015_XX_2353_ARC_4 & 15 & 2.86 & 0.26 \\
A_00PF1_015_XX_2355_ARC_4 & 17 & 3.23 & 0.30 \\
A_00PF1_015_XX_2357_ARC_4 & 19 & 3.03 & 0.27 \\
A_00PF1_015_XX_2359_ARC_4 & 21 & 3.41 & 0.32 \\
A_00PF1_015_XX_2361_ARC_4 & 23 & 3.57 & 0.34 \\
A_00PF1_015_XX_2363_ARC_4 & 25 & 4.69 & 0.44 \\
A_00PF1_015_XX_2365_ARC_4 & 27 & 3.51 & 0.34 \\
A_00PF1_015_XX_2367_ARC_4 & 29 & 0.924 & 0.090 \\
A_00PF1_015_XX_2369_ARC_4 & 31 & 0.388 & 0.038 \\
\hline
\end{tabular}




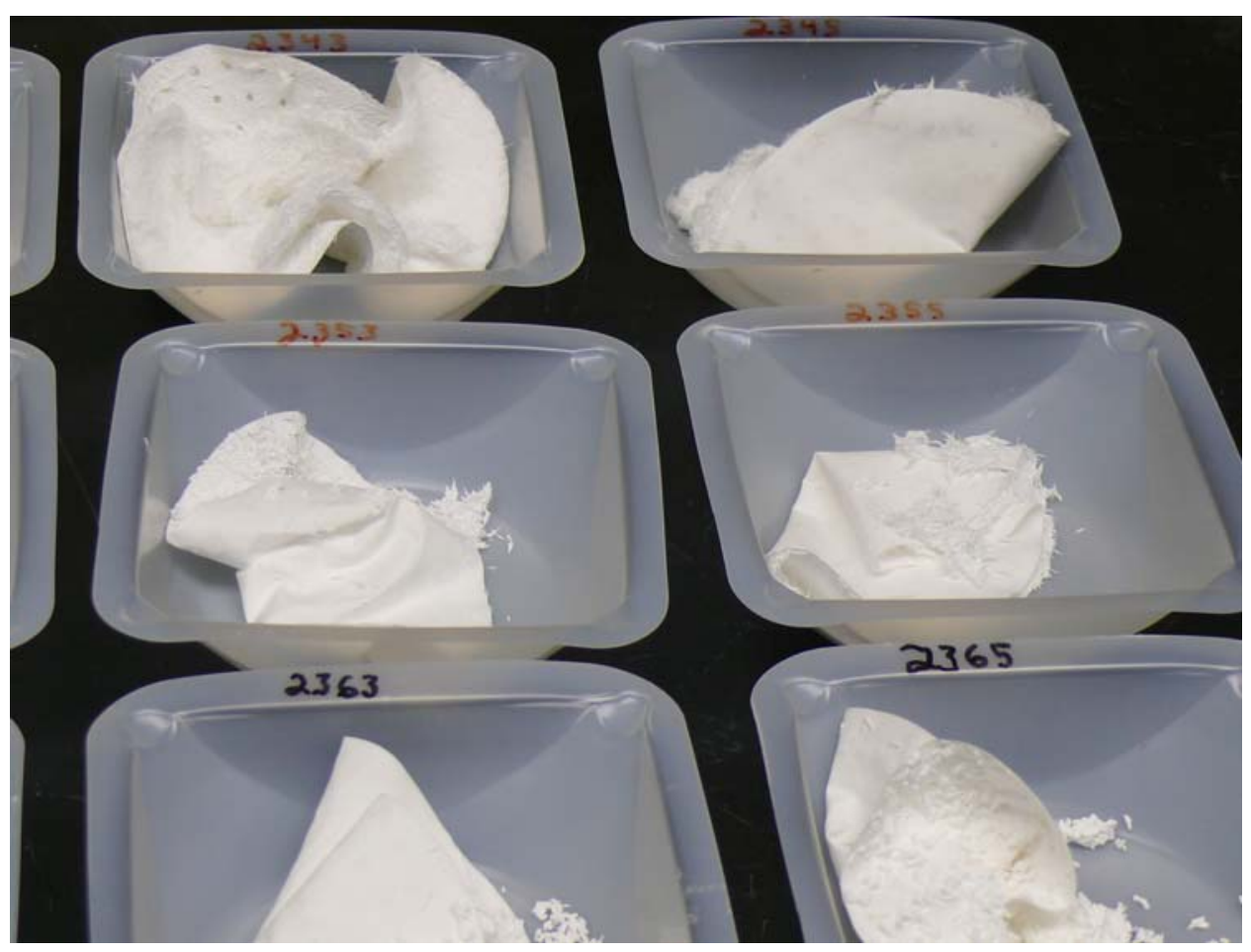

Figure 7.3. Air-Dried Crystals from Various Filtered Odd-Numbered Wash Step Permeate Samples

The even-numbered wash-step permeate samples collected before the slurry sodium ion concentration dropped below 1.8 M (sample \# 2340 to \#2368) all formed crystals covering the bottom of the sample bottle before they could be blended in the correct ratios. Therefore, the bottles were placed in an oven at $80^{\circ} \mathrm{C}$ for 48 hours to re-dissolve the crystals. After the crystals were re-dissolved, 200-mL aliquots of each of these even-numbered wash permeate samples were combined in a single container and stored at room temperature $\left(\sim 18\right.$ to $\left.22^{\circ} \mathrm{C}\right)$ for 10 days. Significant amounts of needle-like crystals were observed in this mixture after 9 days that continued to grow over time as shown in Figure 7.4 and Figure 7.5. These crystals were then filtered through a $0.45-\mu \mathrm{m}$ filter and air-dried as shown in Figure 7.6. There were $34.48 \mathrm{~g}$ of filtered crystals present after drying, which is equivalent to 0.99 -wt $\%$ UDS. After about 5 days, crystals began to form once again in the filtered permeate as shown in Figure 7.7 and appeared to be much smaller crystals than previously.

Another mixture was prepared by taking $200-\mathrm{mL}$ aliquots of each of the same even-numbered wash step permeate samples and combining them in a single container with an equal volume of post-caustic leachate. This 6 -L solution mixture was then stored at room temperature $\left(\sim 18\right.$ to $\left.22^{\circ} \mathrm{C}\right)$ for 10 days. Significant amounts of crystals were observed to form in this solution and appeared to continue to grow over time. These crystals were then filtered through a $0.45-\mu \mathrm{m}$ filter and air-dried. There were $57.72 \mathrm{~g}$ of filtered crystals present after drying, which is equivalent to $0.79-\mathrm{wt} \%$ UDS. These crystals appeared to be smaller and finer than the crystals present in the previous mixture of just the wash-step permeate samples as shown in Figure 7.8 . 


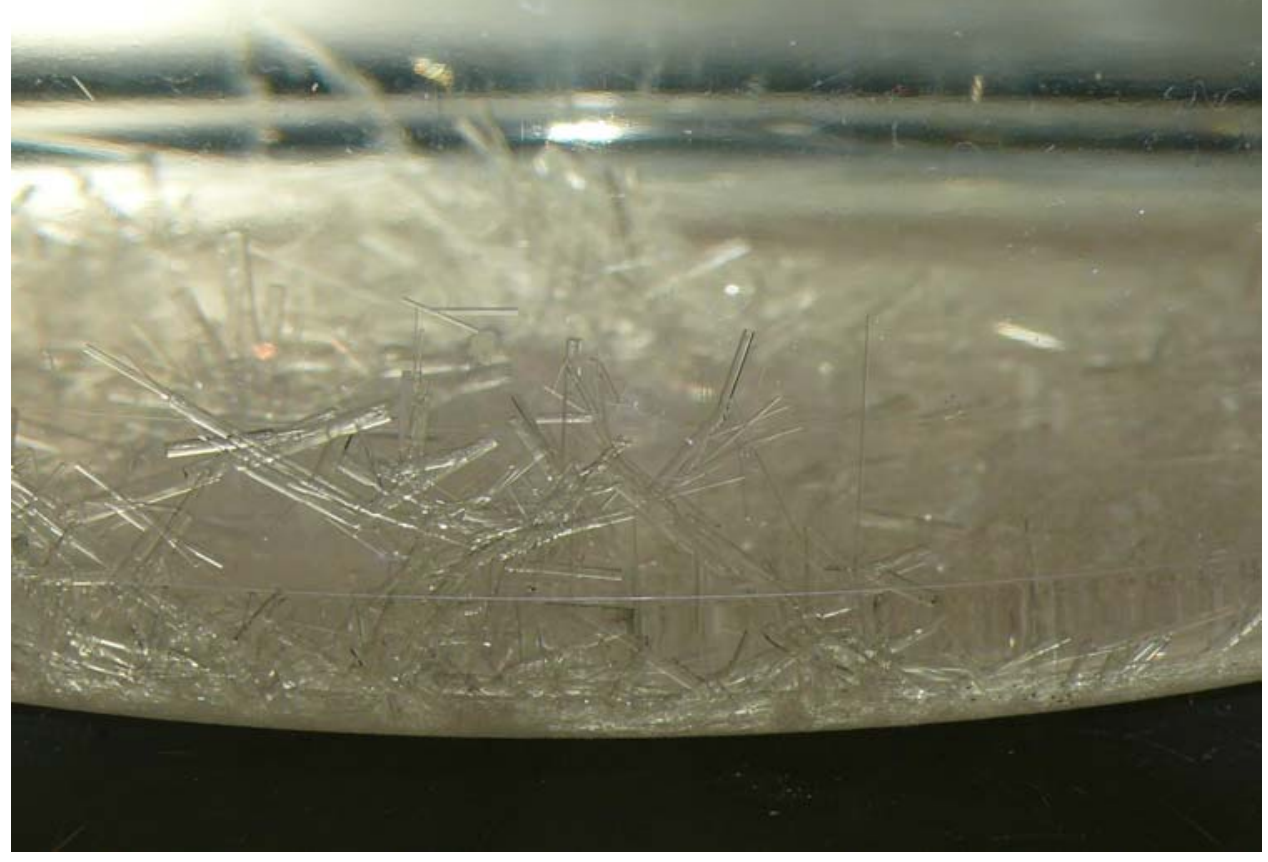

Figure 7.4. Even-Numbered Wash-Step Permeate Blend Crystals

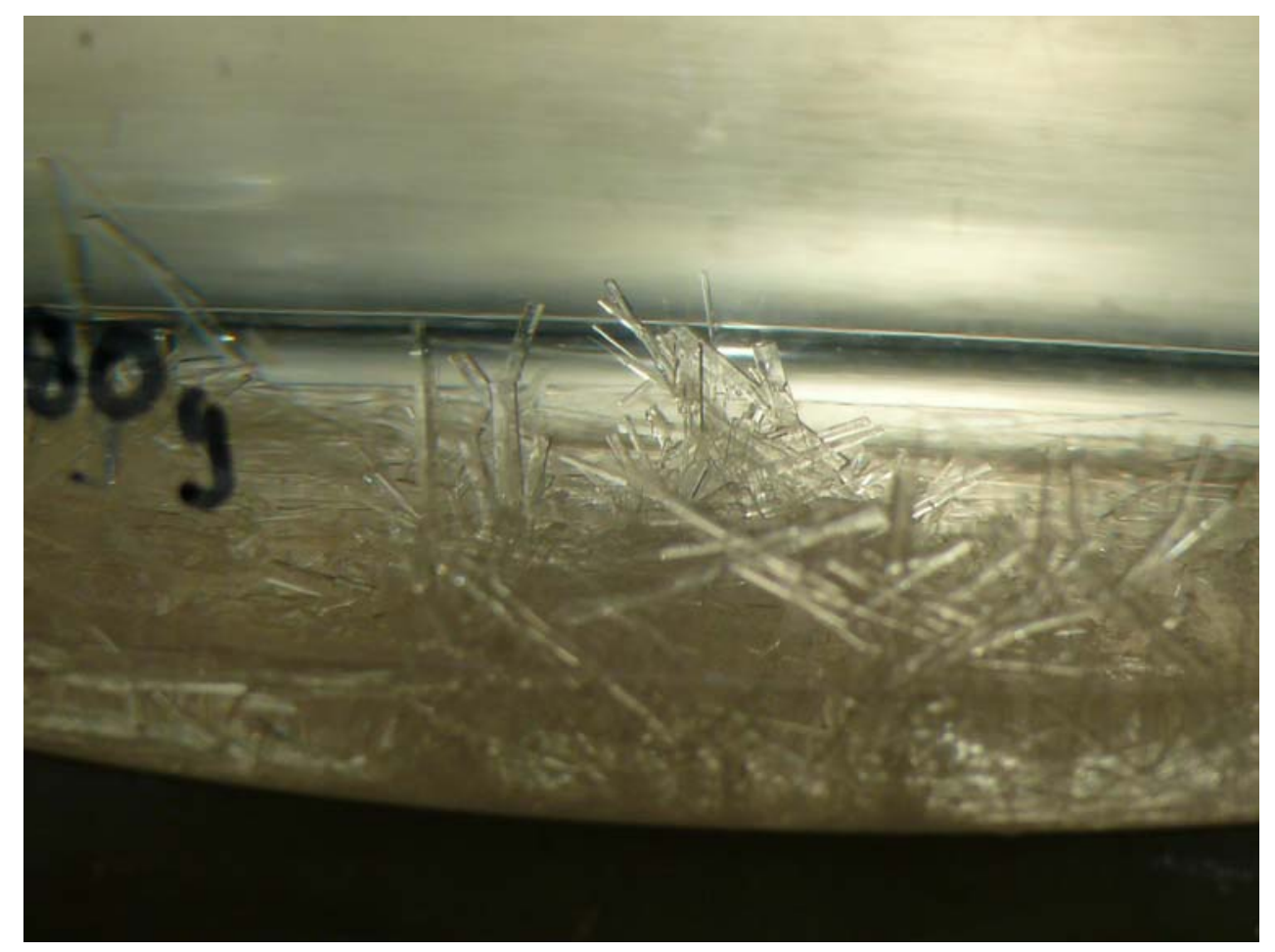

Figure 7.5. More Even-Numbered Wash-Step Permeate Blend Crystals 


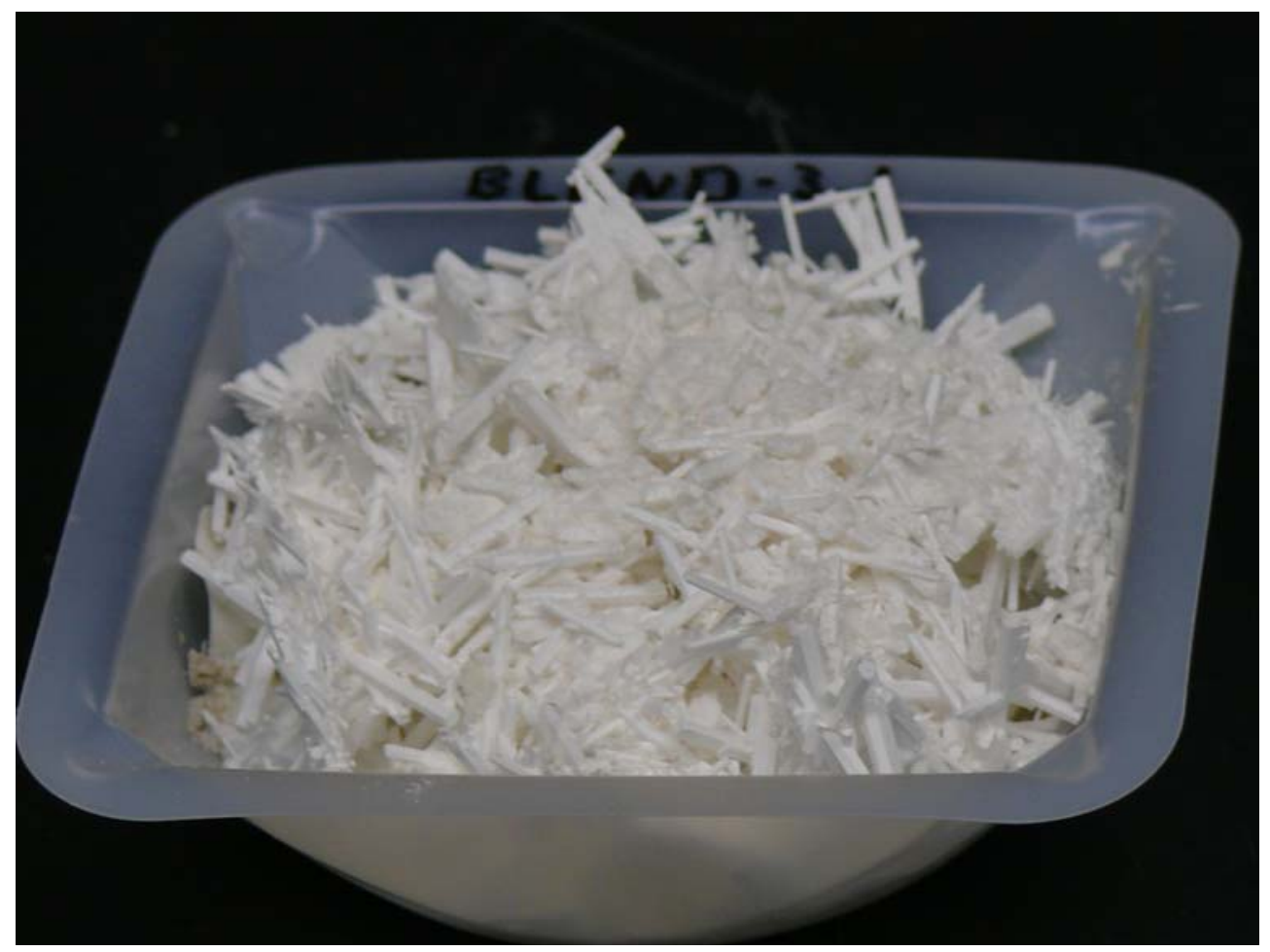

Figure 7.6. Air-Dried Crystals from the Even-Numbered Wash-Step Permeate Mixture

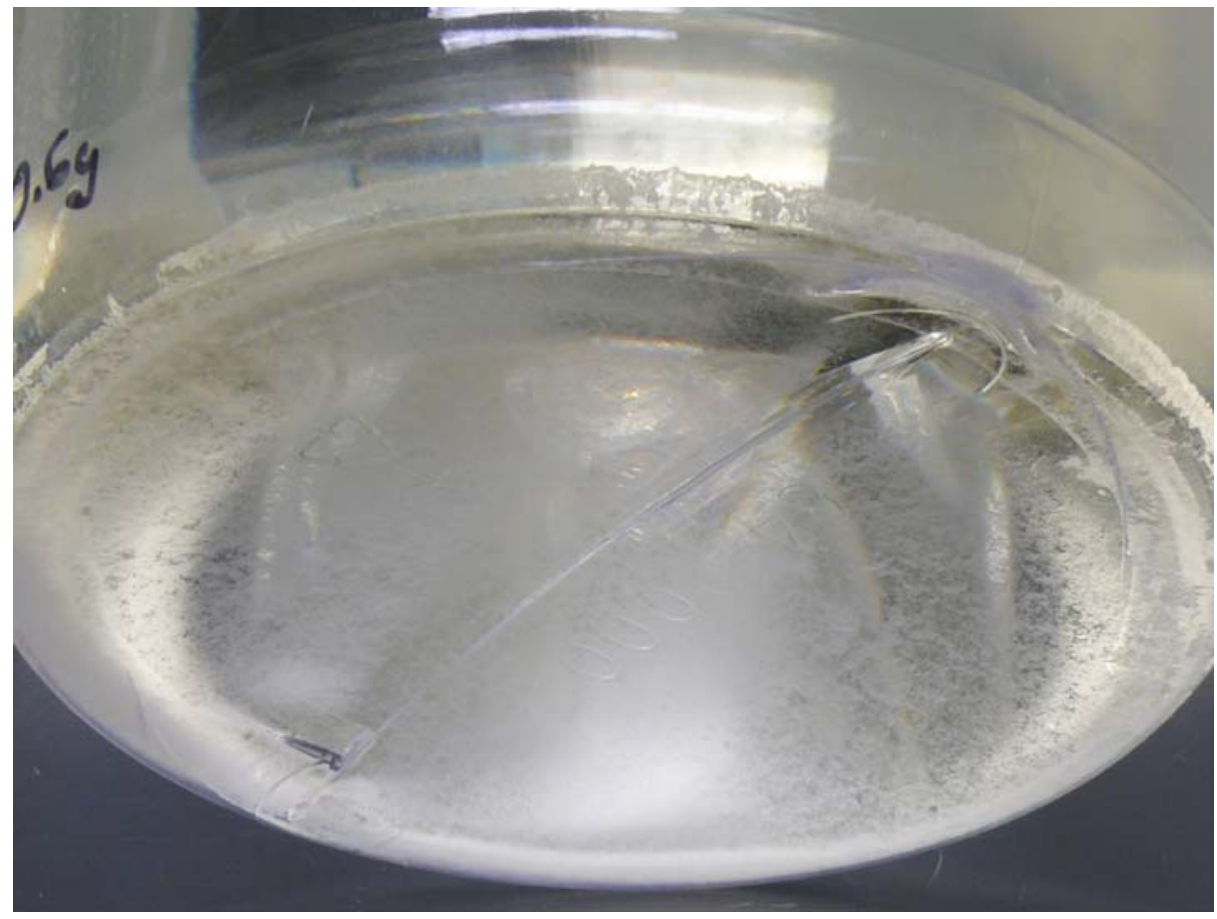

Figure 7.7. Crystals Forming After Filtration of Even-Numbered Wash-Step Permeate Blend 


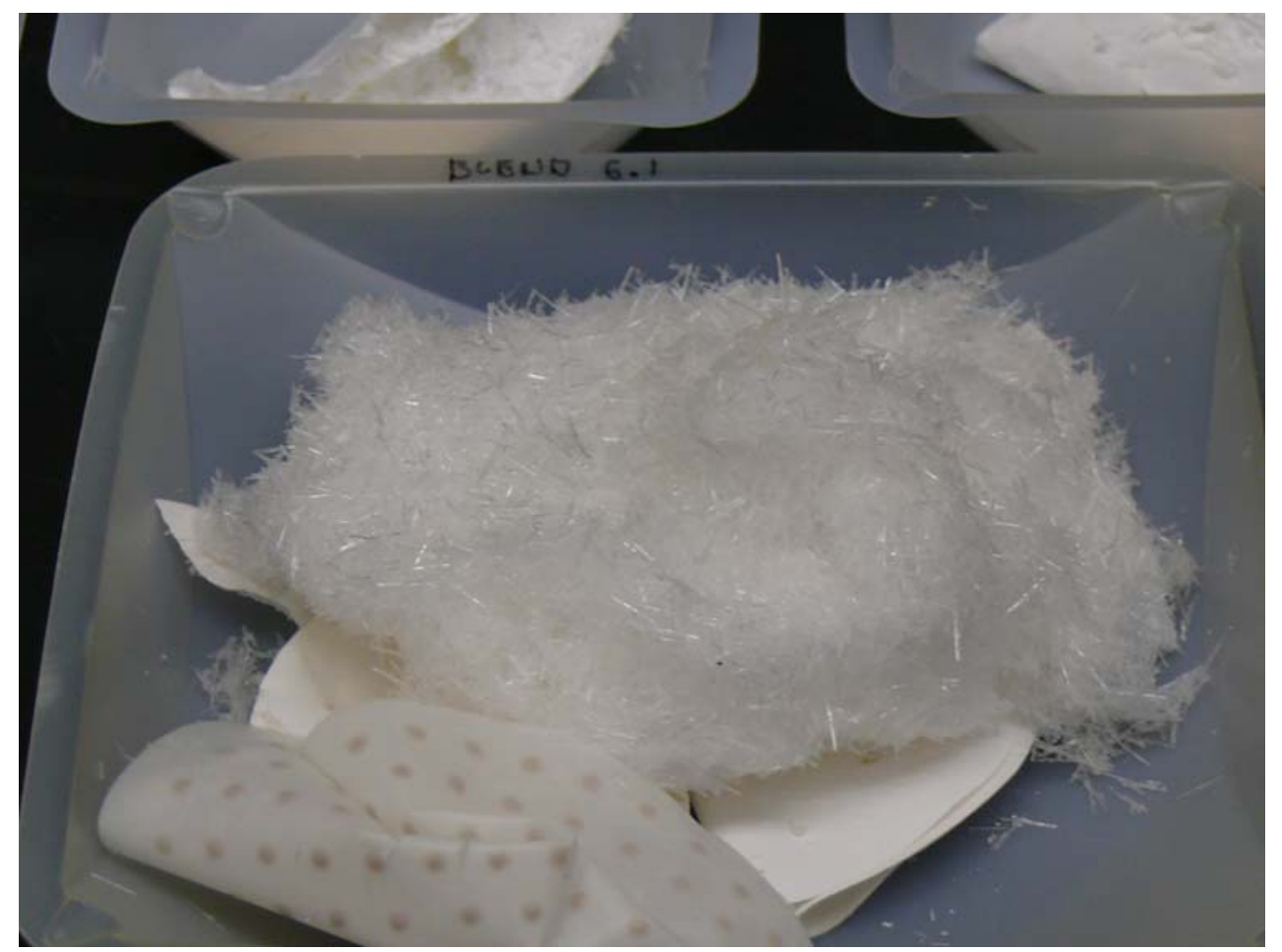

Figure 7.8. Air-Dried Crystals from the 1:1 Wash-Step Permeate: Leachate Mixture

A 2-L permeate sample was collected on February 2, 2009 at 10:01 near the end of the post-caustic-leach solids concentration process for Test A (Sample ID \#

A_00PF1_007_XX_2555_ARC_4). This sample was maintained at laboratory temperature $\left(\sim 18\right.$ to $\left.22^{\circ} \mathrm{C}\right)$ for about 3 months. During the first 2 months, no precipitate appeared. However, during the last month, several needle-like crystals grew in the sample as seen in Figure 7.9. These crystals were then filtered, air-dried, and weighed. There were $2.88 \mathrm{~g}$ of crystals present, which is equivalent to $0.26-\mathrm{wt} \%$.

A 2-L permeate sample was collected near the end of the initial solids concentration processes in Tests B and D. These samples were maintained at laboratory temperature $\left(\sim 18\right.$ to $\left.22^{\circ} \mathrm{C}\right)$ for about 2 months. No precipitates were observed during this timeframe in either sample. Only the PEP Test D washes and leachate were looked at with PLM and had particle size distribution measured from the PLM photos. The PEP Tests B and D leachates were not analyzed during this testing. What analysis was performed on these leachates will be reported in WTP-RPT-205. ${ }^{(a)}$

(a) RL Russell, RA Peterson, DE Rinehart, and JV Crum. 2009. Results of the Laboratory Precipitate Study from PEP Simulant. WTP-RPT-205, Rev. A, Bechtel National Inc., Richland, Washington. 


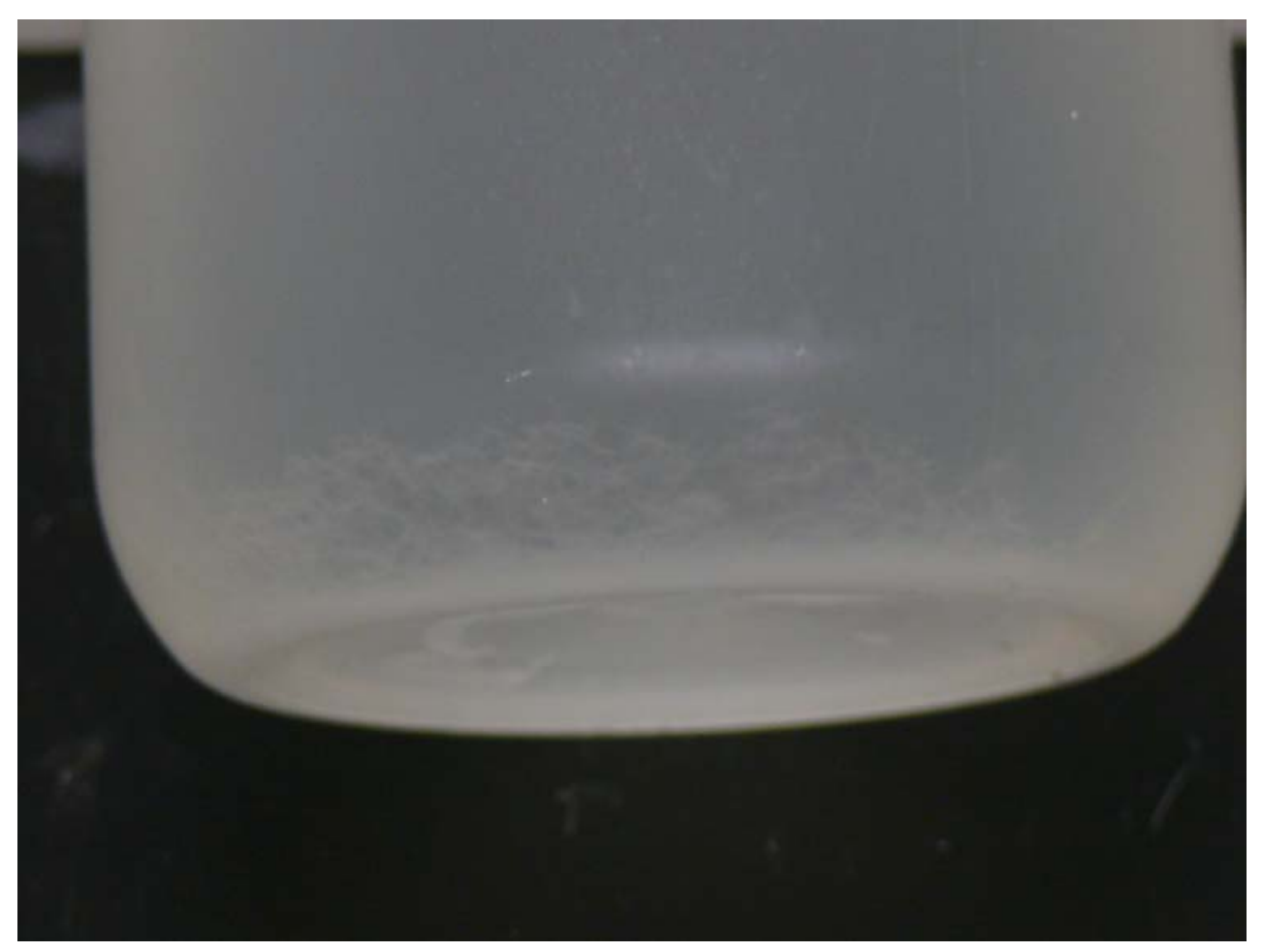

Figure 7.9. Crystals in Test A Initial Solids Concentration Permeate

PEP Test D post-caustic-leach water washing began on March 30, 2009 at 3:48 and continued until 12:00 using 53 wash steps. A 1-liter permeate sample was taken with each wash step and then transferred to a heated trailer that was kept at $80 \pm 1^{\circ} \mathrm{F}$.

Four samples from PEP Test D post-caustic-leach water washing were examined with polarized light microscopy (PLM) to determine particle size and composition of the precipitate that had formed. Pictures of crystals in the very small samples withdrawn from the wash permeate PEP samples were taken after the samples had been at laboratory temperature $\left(\sim 18\right.$ to $\left.22^{\circ} \mathrm{C}\right)$ for 6 days and 4 hours without mixing. A couple of drops of solution with crystals present were removed from the bottle using a large opening pipette and placed on a microscope slide.

To determine the particle composition, the microscope slides were placed on the polarized light microscope after 8 days and 21 hours without mixing and compared to the standard polarized light microscope pictures of sodium phosphate and sodium oxalate. These pictures show that the crystals were mainly sodium phosphate with some sodium oxalate, as shown in Figure 7.10 through Figure 7.13 by the standard PLM photos included next to the figure.

To determine particle size, several pictures at 50× magnification on a non-polarized light microscope were taken at different areas of the microscope slide. These pictures were scaled, and each particle on the pictures was measured for a total of approximately 100 particles. Pictures were taken again after another week ( 6 days and 20.5 hours) to determine the crystal growth in the samples. The crystal size growth is shown in Figure 7.14 through Figure 7.17 by plotting the cumulative particle size measured from the photographs and given in tabular form in Table 7.3 through Table 7.6. Two samples, Sample Bottle \#D_00PF1_015_XX_5444_ARC_4 (wash \#8) and Sample Bottle \#D_00PF1_015_XX_5476_ARC_4 
(wash \#40), appeared to decrease in size over time because the crystals had to be broken up to remove them from the sample bottle and prepare a microscope slide. Therefore, this is probably not an accurate assessment of the crystal growth in these samples. The other samples continued to show the particle size increasing over time.

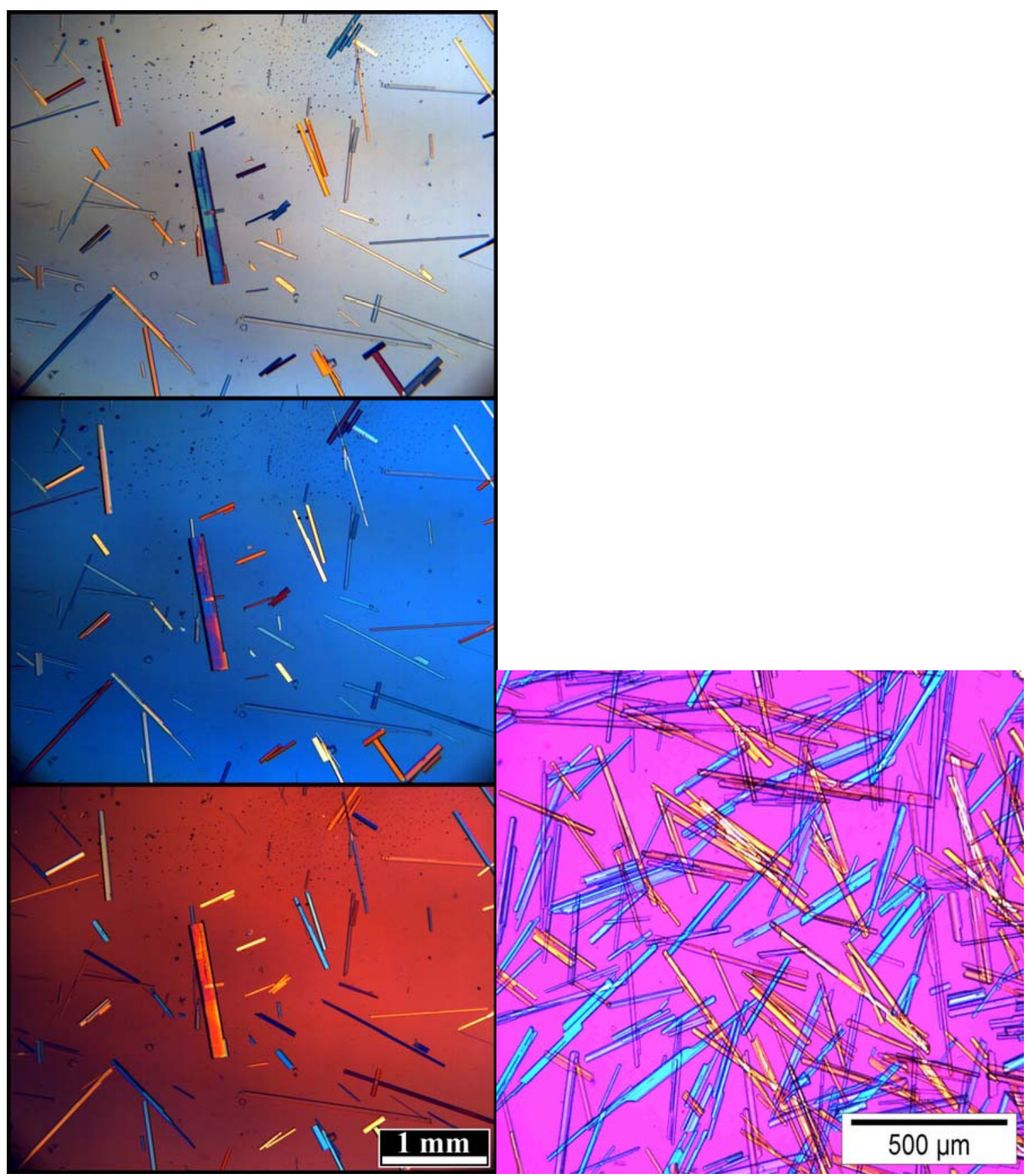

Figure 7.10. Polarized Light Picture of Sample Bottle \#D_00PF1_015_XX_5653_ARC_4 (post-caustic-leach dewater) with a Standard $\mathrm{Na}_{3} \mathrm{PO}_{4}$ Picture Beside it 


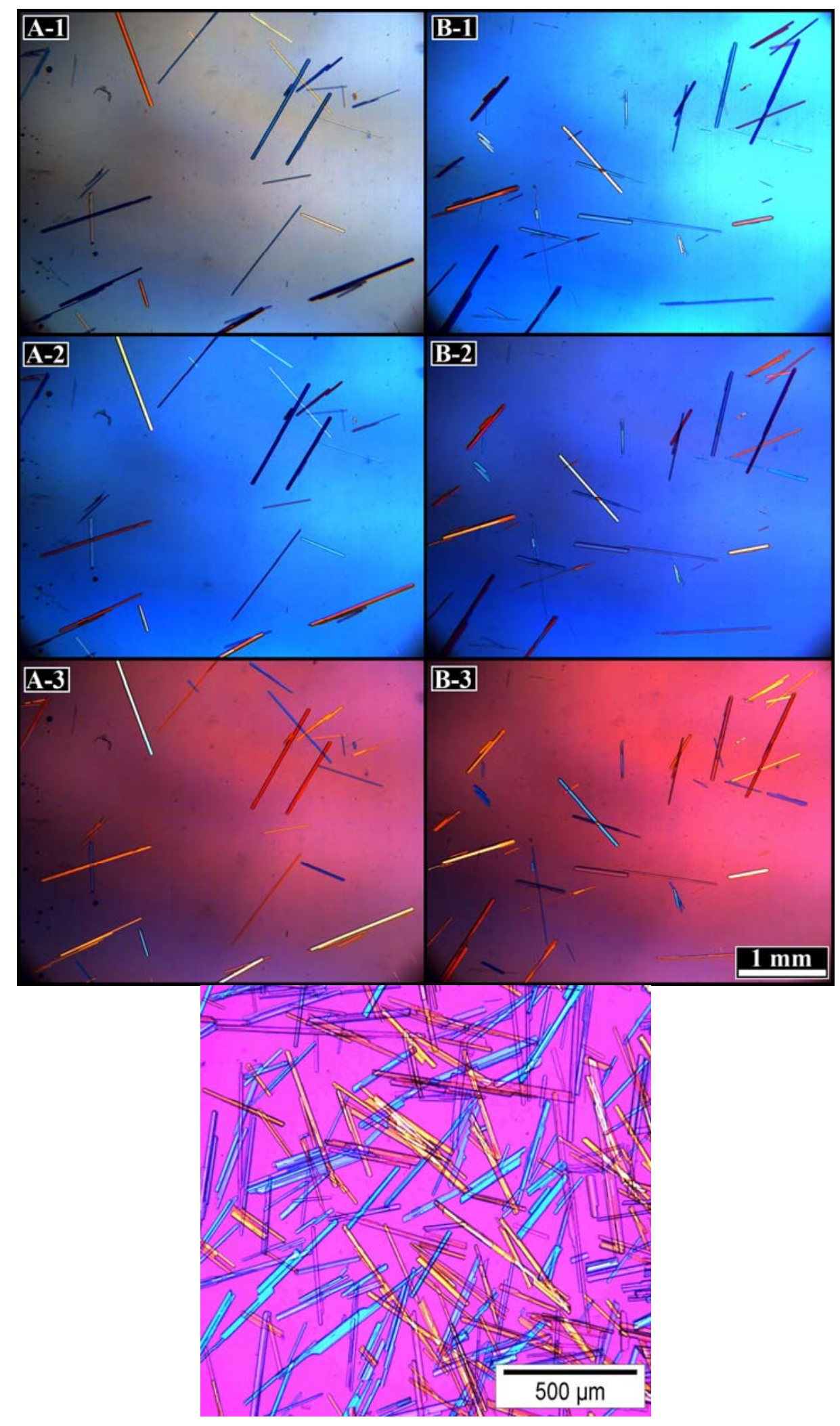

Figure 7.11. Polarized Light Picture of Sample Bottle \#D_00PF1_015_XX_5444_ARC_4 (wash \#8) with a Standard $\mathrm{Na}_{3} \mathrm{PO}_{4}$ Picture Under it 


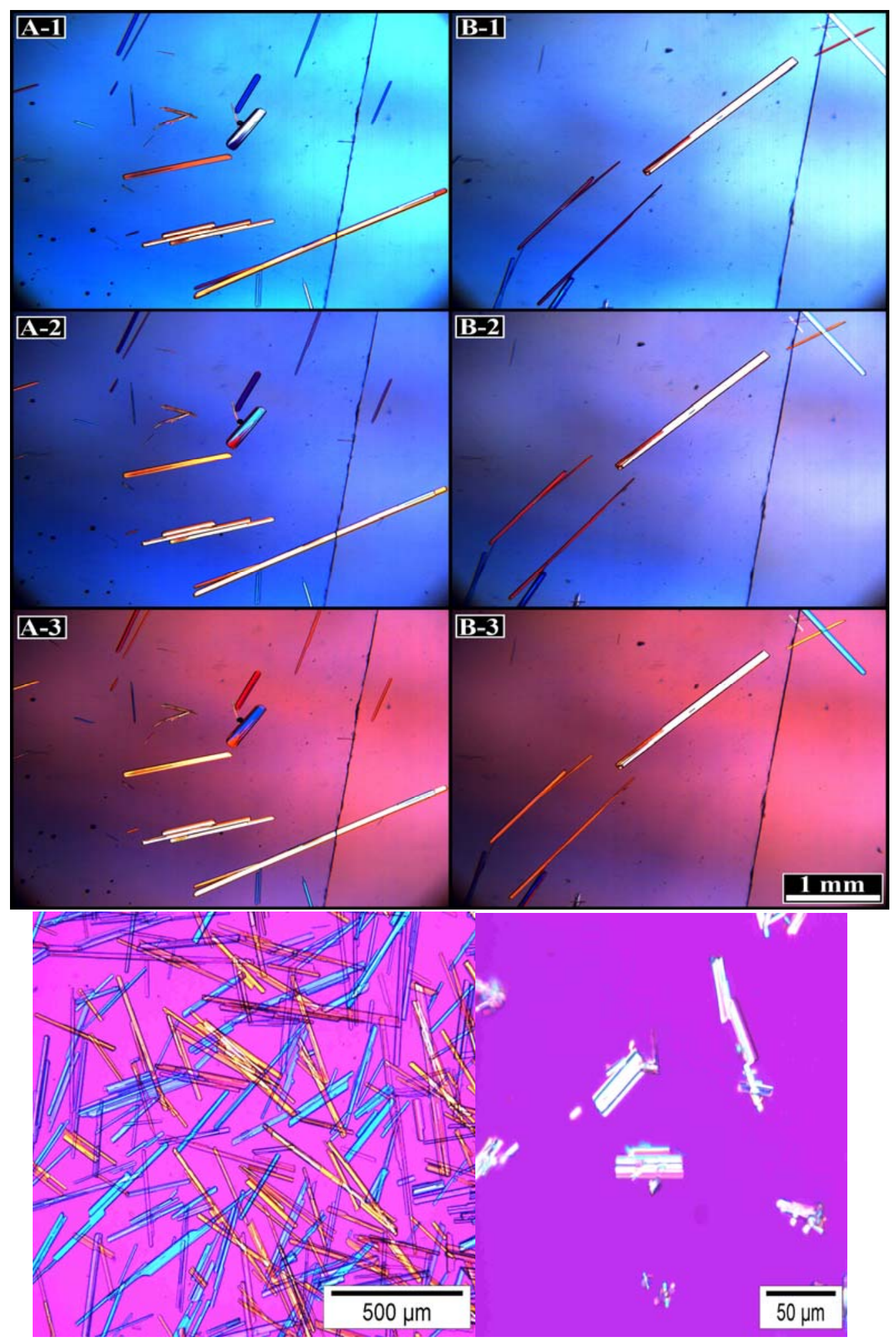

Figure 7.12. Polarized Light Picture of Sample Bottle \#D_00PF1_015_XX_5675_ARC_4 (wash \#13) with a Standard $\mathrm{Na}_{3} \mathrm{PO}_{4}$ Picture (on the left) and a Standard $\mathrm{Na}_{2} \mathrm{C}_{2} \mathrm{O}_{4}$ Picture (on the right) Under it 


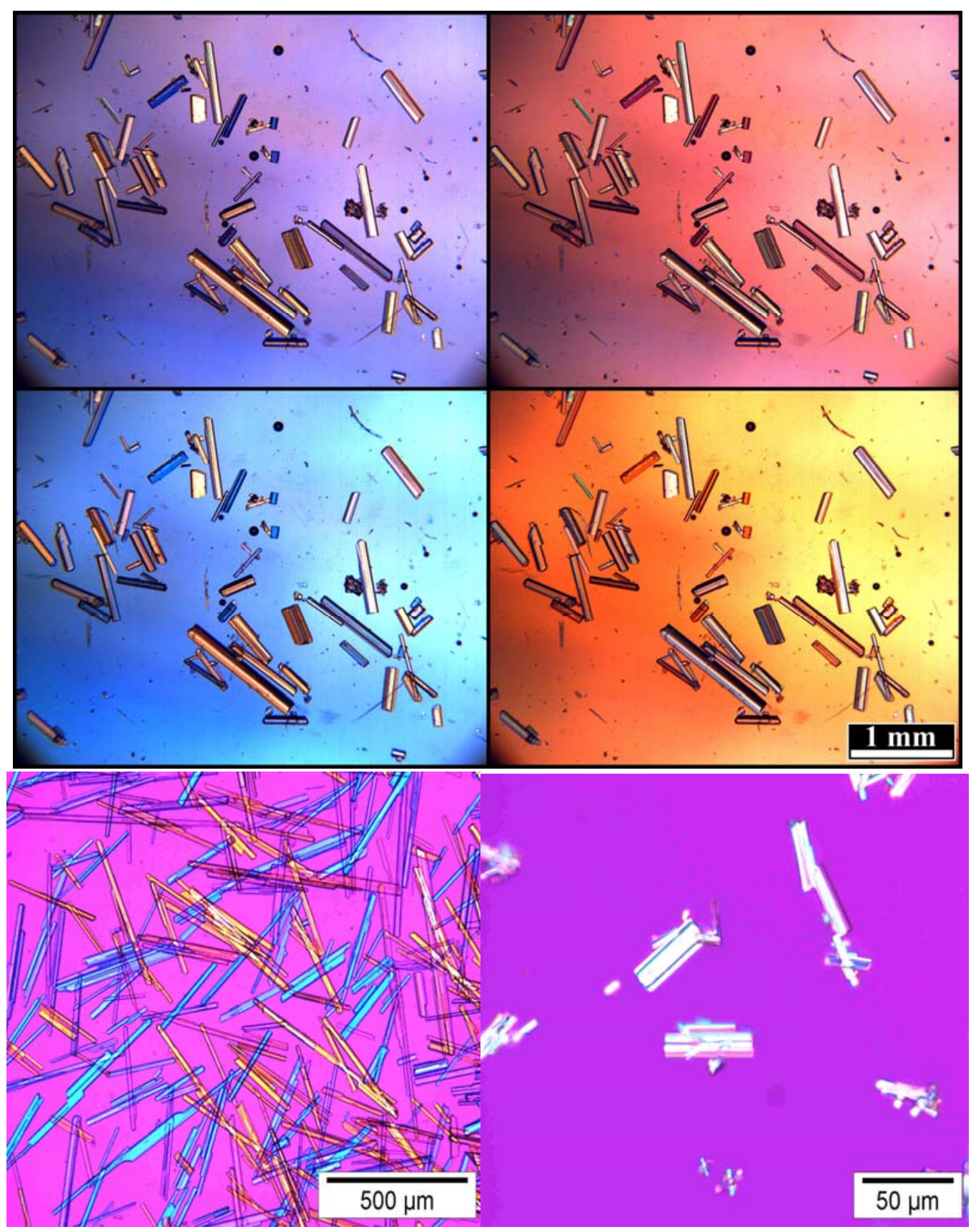

Figure 7.13. Polarized Light Picture of Sample Bottle \#D_00PF1_015_XX_5476_ARC_4 (wash \#40) with a Standard $\mathrm{Na}_{3} \mathrm{PO}_{4}$ Picture (on the left) and a Standard $\mathrm{Na}_{2} \mathrm{C}_{2} \mathrm{O}_{4}$ Picture (on the right) Under it 


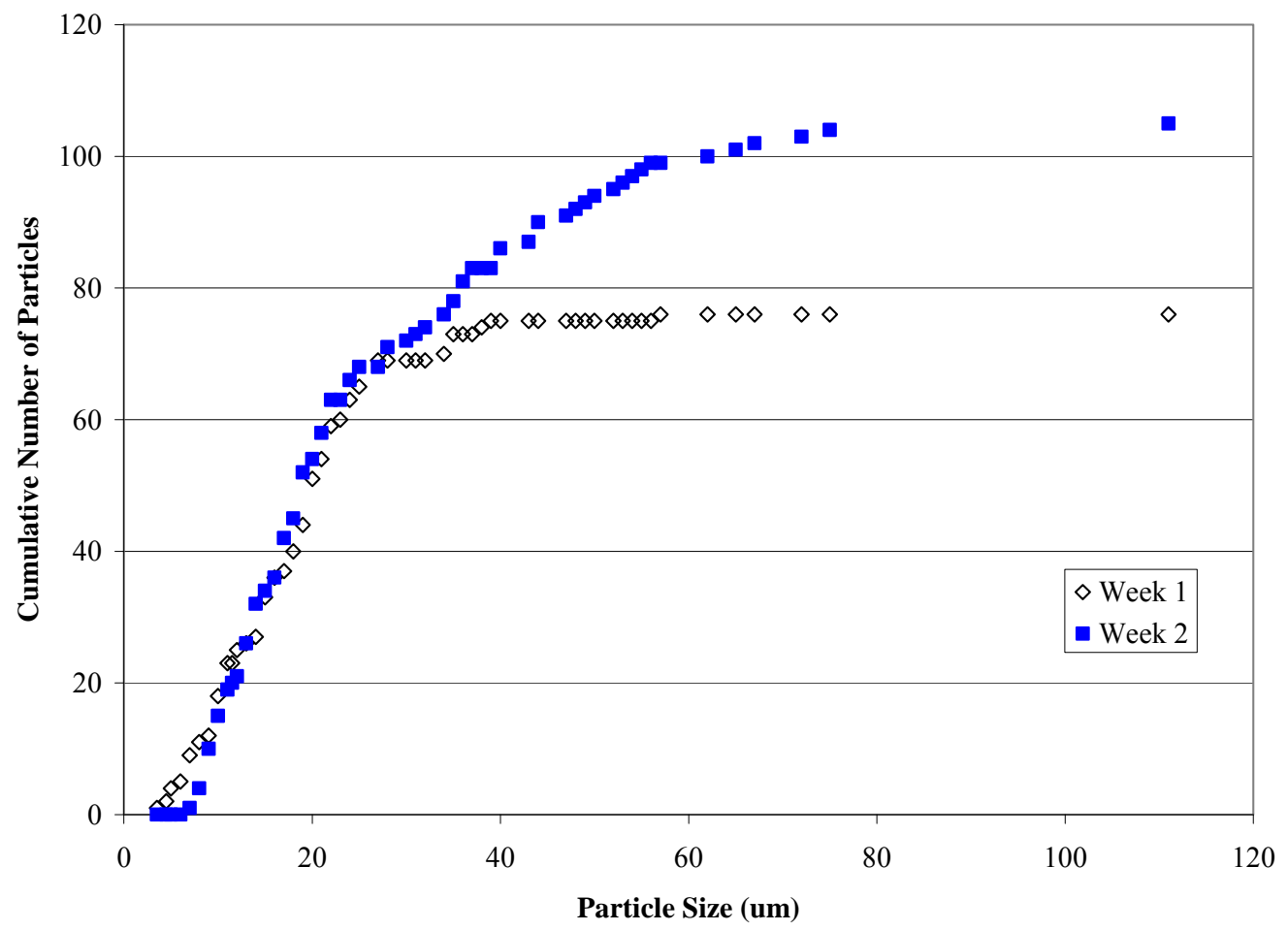

Figure 7.14. Particle Length Distribution of Sample Bottle \#D_00PF1_015_XX_5653_ARC_4 (post-caustic-leach dewater)

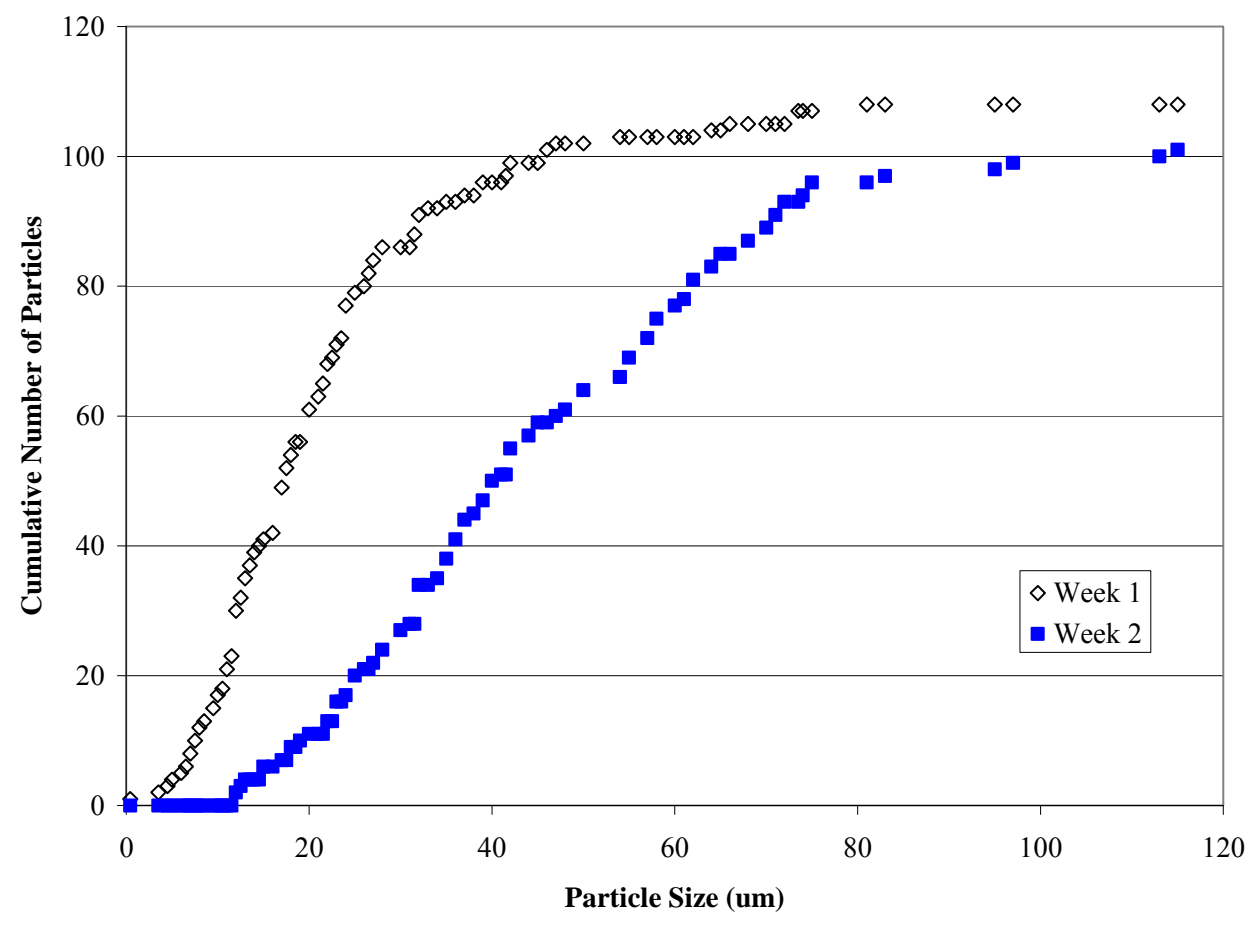

Figure 7.15. Particle Length Distribution of Sample Bottle \#D_00PF1_015_XX_5444_ARC_4 (wash \#8) 


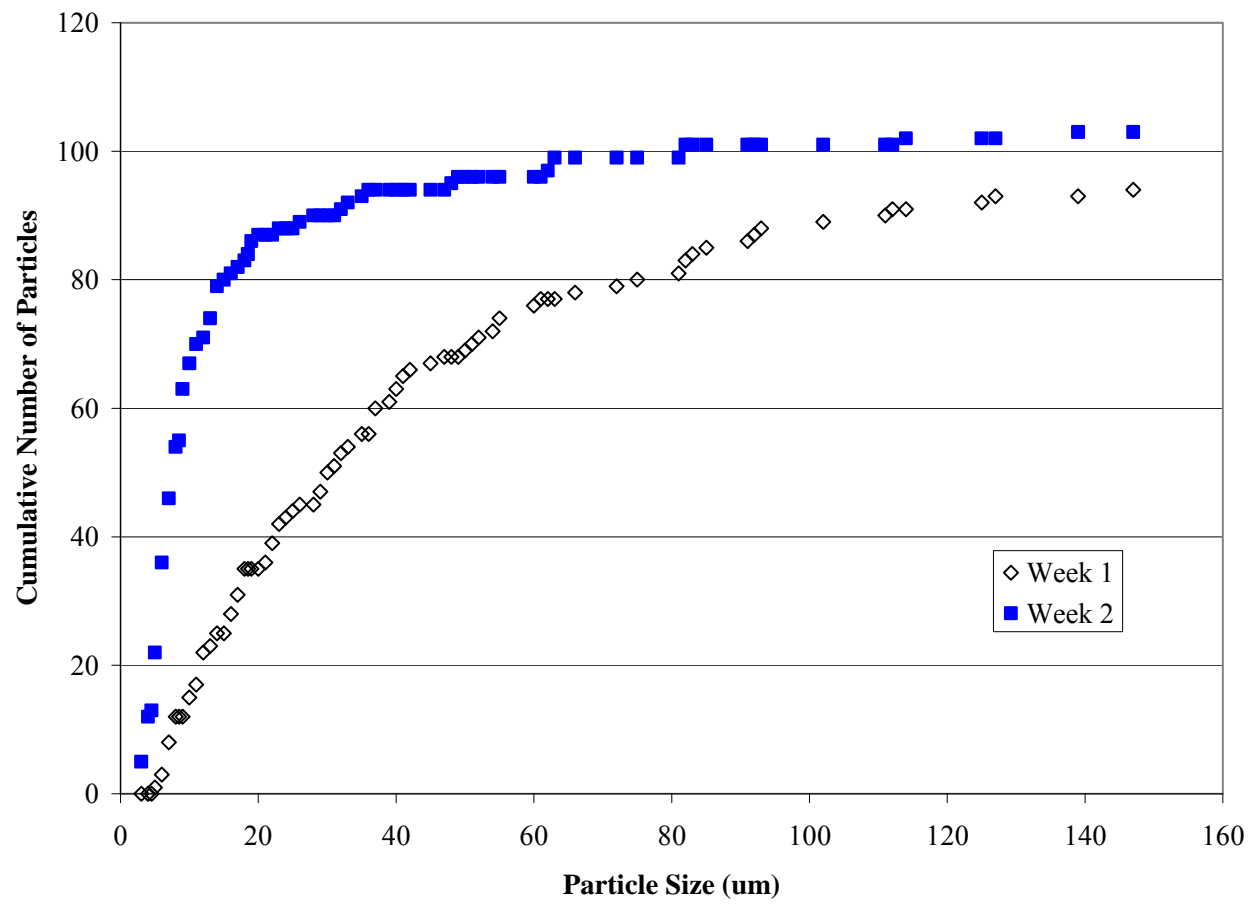

Figure 7.16. Particle Length Distribution of Sample Bottle \#D_00PF1_015_XX_5675_ARC_4 (wash \#13)

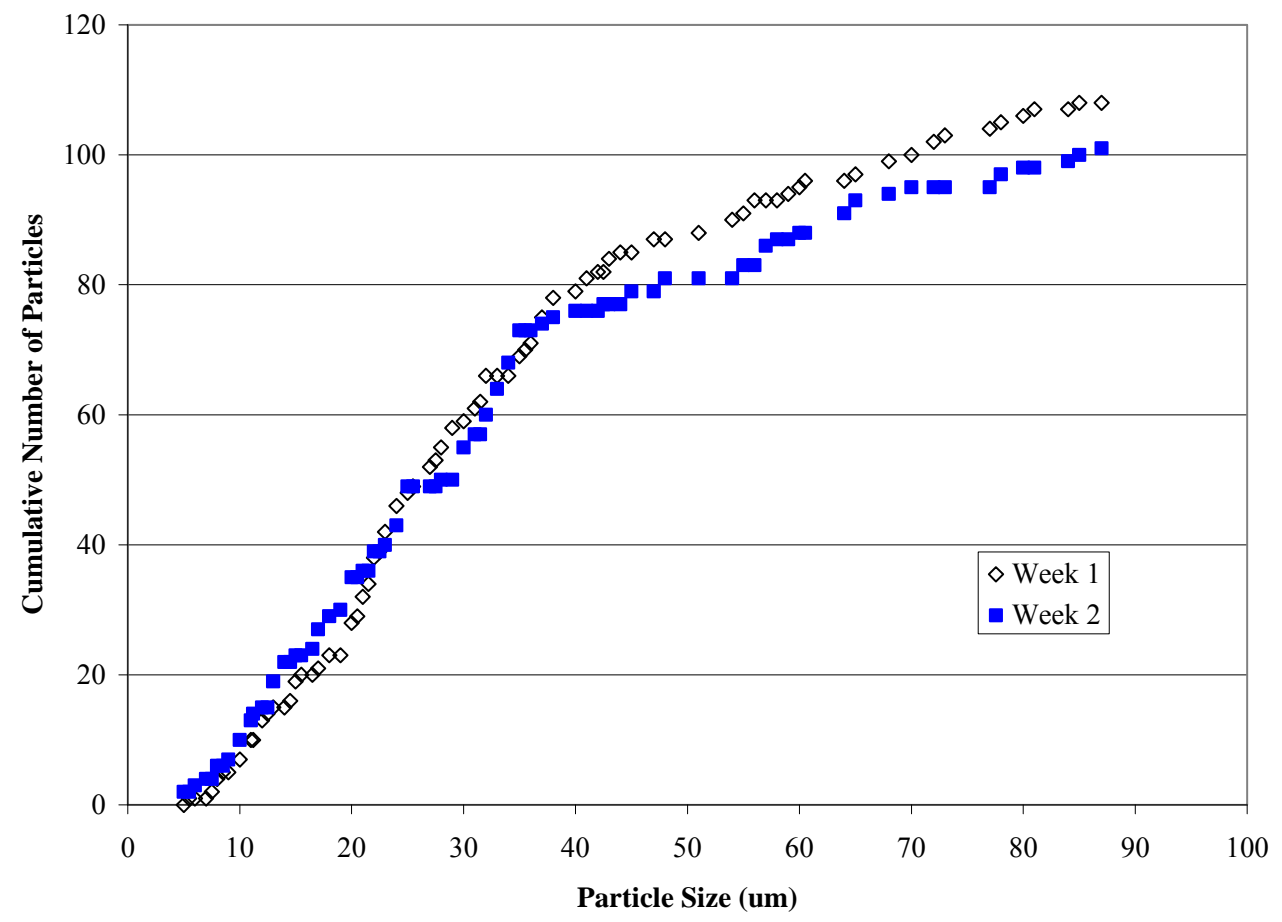

Figure 7.17. Particle Length Distribution of Sample Bottle \#D_00PF1_015_XX_5476_ARC_4 (wash \#40) 
Table 7.3. PSD for Sample 5653

\begin{tabular}{|c|c|c|c|c|c|c|c|}
\hline \multicolumn{8}{|c|}{ Data in $\mathrm{mm}$} \\
\hline \multicolumn{5}{|c|}{ Week $1(100 \times$ and $200 \times$ magnification $)$} & \multicolumn{3}{|c|}{ Week $2(50 \times$ magnification $)$} \\
\hline $5653-1$ & $5653-2$ & $5653-3$ & $5653-4$ & $5653-5$ & $5653-1$ & $5653-2$ & $5653-3$ \\
\hline 39 & 10 & 7 & 12 & 24 & 18 & 47 & 9 \\
\hline 21 & 14 & 11 & 11 & 38 & 17 & 67 & 21 \\
\hline 34 & 15 & 35 & 15 & 23 & 11 & 24 & 72 \\
\hline 27 & 9 & 7 & 10 & 10 & 50 & 11 & 37 \\
\hline 20 & 15 & 21 & 12 & 25 & 17 & 36 & 28 \\
\hline \multirow[t]{33}{*}{22} & 19 & 5 & 24 & 8 & 35 & 54 & 13 \\
\hline & 17 & 10 & 21 & 15 & 9 & 10 & 30 \\
\hline & 19 & 20 & 18 & 16 & 14 & 36 & 15 \\
\hline & 13 & 5 & 22 & 24 & 24 & 19 & 31 \\
\hline & 18 & 11 & 11 & 22 & 24 & 53 & 10 \\
\hline & 25 & 7 & 18 & 19 & 65 & 21 & 48 \\
\hline & 11 & 4.5 & 22 & 20 & 10 & 18 & 9 \\
\hline & 22 & 3.5 & 27 & 10 & 34 & 19 & 22 \\
\hline & 16 & & 10 & 27 & 40 & 8 & 15 \\
\hline & 8 & & 6 & 35 & 36 & 11.5 & \\
\hline & & & 27 & 20 & 16 & 62 & \\
\hline & & & 15 & 35 & 21 & 20 & \\
\hline & & & 15 & 20 & 75 & 7 & \\
\hline & & & 7 & 19 & 11 & 17 & \\
\hline & & & & 57 & 25 & 32 & \\
\hline & & & & 20 & 28 & 17 & \\
\hline & & & & 20 & 43 & 44 & \\
\hline & & & & 16 & 17 & 35 & \\
\hline & & & & & 17 & 44 & \\
\hline & & & & & 22 & 56 & \\
\hline & & & & & 40 & 12 & \\
\hline & & & & & 25 & 9 & \\
\hline & & & & & 14 & 8 & \\
\hline & & & & & 34 & 19 & \\
\hline & & & & & 11 & 18 & \\
\hline & & & & & 9 & 13 & \\
\hline & & & & & 20 & 9 & \\
\hline & & & & & 37 & 13 & \\
\hline & & & & & 49 & 111 & \\
\hline & & & & & 28 & 22 & \\
\hline & & & & & 13 & 55 & \\
\hline & & & & & 10 & 44 & \\
\hline & & & & & 14 & 19 & \\
\hline
\end{tabular}


Table 7.3. PSD for Sample 5653

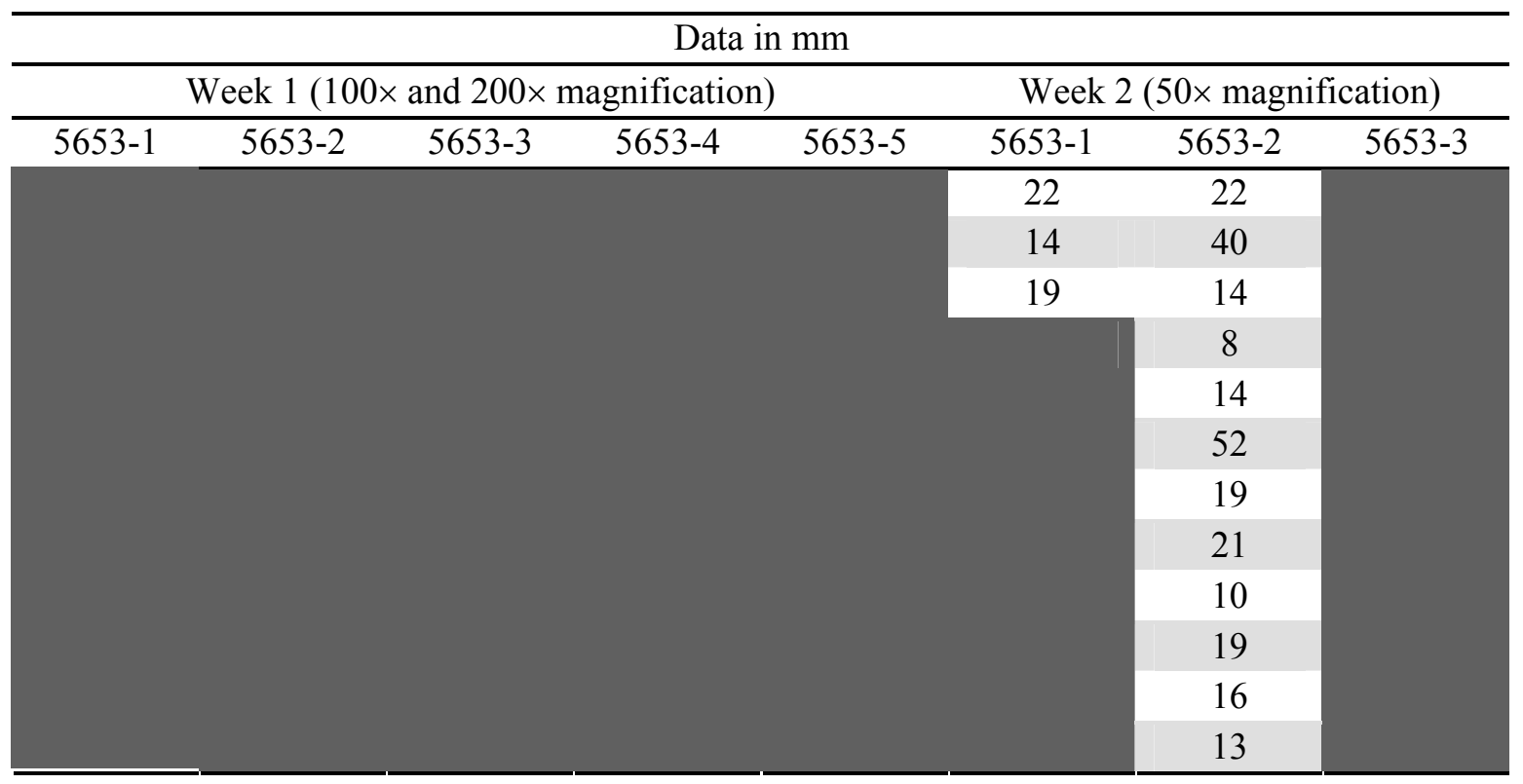


Table 7.4. PSD for Sample 5444

\begin{tabular}{|c|c|c|c|c|c|c|c|}
\hline \multicolumn{8}{|c|}{ Data in $\mathrm{mm}(50 \times$ magnification $)$} \\
\hline \multicolumn{5}{|c|}{ Week 1} & \multicolumn{3}{|c|}{ Week 2} \\
\hline $5444-1$ & $5444-2$ & $5444-3$ & $5444-4$ & $5444-5$ & $5444-1$ & $5444-2$ & $5444-3$ \\
\hline 12 & 14.5 & 8 & 7 & 6.5 & 28 & 12.5 & 28 \\
\hline 23 & 9.5 & 11.5 & 32 & 16 & 37 & 26 & 22 \\
\hline 15 & 10 & 14 & 12 & 7 & 65 & 15 & 19 \\
\hline 17 & 12 & 73.5 & 17 & 4.5 & 50 & 62 & 50 \\
\hline 12 & 3.5 & 23 & 17 & 5 & 23 & 40 & 24 \\
\hline 11 & 41.5 & 7.5 & 20 & 26.5 & 25 & 60 & 25 \\
\hline 12 & 12 & 31.5 & 54 & 13.5 & 83 & 31 & 47 \\
\hline 17.5 & 17 & 28 & 22 & 24 & 71 & 50 & 22 \\
\hline 22.5 & 35 & 21.5 & 27 & 9.5 & 36 & 58 & 37 \\
\hline 20 & 25 & 46 & 8 & 26 & 45 & 42 & 64 \\
\hline 26.5 & 32 & 12.5 & 20 & 8.5 & 42 & 64 & 39 \\
\hline 73.5 & 17 & 46 & 28 & 17 & 40 & 42 & 36 \\
\hline 11.5 & 81 & 21 & 13 & 18.5 & 54 & 30 & 32 \\
\hline 20 & 47 & 6 & 18.5 & 13 & 44 & 42 & 72 \\
\hline 0.42 & 39 & 10 & 10.5 & 24 & 23 & 54 & 115 \\
\hline 24 & 64 & 11 & 24 & 18 & 27 & 39 & 75 \\
\hline 23.5 & 42 & 42 & 27 & 66 & 61 & 58 & 70 \\
\hline 20 & 12.5 & 22 & 21.5 & 32 & 97 & 57 & 75 \\
\hline 33 & 37 & 18 & 22 & 13 & 50 & 32 & 68 \\
\hline 25 & 21 & 12 & 31.5 & 17 & 72 & 113 & 12 \\
\hline & 39 & & 24 & 17.5 & 65 & 45 & 13 \\
\hline & 11 & & 13.5 & 14 & 70 & 60 & 18 \\
\hline & & & 17.5 & 7.5 & 55 & 55 & 95 \\
\hline & & & & & 48 & 35 & 55 \\
\hline & & & & & 20 & 68 & 35 \\
\hline & & & & & 37 & 35 & 32 \\
\hline & & & & & 25 & 57 & 15 \\
\hline & & & & & 71 & 30 & 38 \\
\hline & & & & & 57 & 32 & 32 \\
\hline & & & & & 58 & 23 & 30 \\
\hline & & & & & 62 & 36 & \\
\hline & & & & & 12 & 74 & \\
\hline & & & & & 44 & 62 & \\
\hline & & & & & 40 & 41 & \\
\hline & & & & & 32 & 18 & \\
\hline & & & & & 34 & & \\
\hline & & & & & 17 & & \\
\hline
\end{tabular}


Table 7.5. PSD for Sample 5675

\begin{tabular}{|c|c|c|c|c|c|c|c|c|c|}
\hline \multicolumn{10}{|c|}{ Data in $\mathrm{mm}(50 \times$ magnification $)$} \\
\hline \multicolumn{5}{|c|}{ Week 1} & \multicolumn{5}{|c|}{ Week 2} \\
\hline $5675-1$ & $5675-2$ & $5675-3$ & $5675-4$ & $5675-5$ & $5675-1$ & $5675-2$ & $5675-3$ & $5675-4$ & $5675-5$ \\
\hline 17 & 18 & 7 & 42 & 22 & 3 & 48 & 32 & 5 & 4 \\
\hline 51 & 12 & 30 & 12 & 82 & 9 & 49 & 18.5 & 4 & 11 \\
\hline 7 & 29 & 23 & 125 & 72 & 33 & 14 & 6 & 28 & 4 \\
\hline 10 & 92 & 39 & 18 & 6 & 20 & 5 & 13 & 7 & 4 \\
\hline 6 & 11 & 16 & 60 & 30 & 6 & 9 & 23 & 14 & 4 \\
\hline 8 & 55 & 8 & 32 & 14 & 6 & 5 & 7 & 4 & 139 \\
\hline 33 & 75 & 24 & 41 & 22 & 11 & 7 & 10 & 9 & 7 \\
\hline 12 & 12 & 83 & 35 & 30 & 3 & 11 & 26 & 7 & 6 \\
\hline 22 & 45 & 17 & 37 & 32 & 6 & 7 & 9 & 10 & 5 \\
\hline 14 & 66 & 21 & 8 & 11 & 6 & 8 & 63 & 14 & 12 \\
\hline 7 & 29 & 18 & 10 & 41 & 6 & 7 & 9 & 8 & 6 \\
\hline 5 & 91 & 93 & 7 & & 4.5 & 5 & 5 & 13 & 62 \\
\hline 10 & 81 & 40 & 7 & & 9 & 6 & 3 & 10 & 16 \\
\hline 52 & 31 & 102 & 47 & & 6 & 7 & & 8 & 9 \\
\hline 37 & 60 & 127 & 40 & & 7 & 6 & & 5 & 35 \\
\hline 111 & 85 & 147 & 35 & & 82 & 9 & & 5 & 14 \\
\hline 18 & 112 & 25 & 37 & & 14 & & & 6 & 6 \\
\hline 23 & 50 & 16 & 82 & & 15 & & & 3 & 36 \\
\hline 16 & 55 & 61 & & & 8 & & & 13 & 19 \\
\hline 37 & 12 & 54 & & & 6 & & & 8.5 & 10 \\
\hline 8 & & 23 & & & 82 & & & 5 & 63 \\
\hline 26 & & & & & 114 & & & 4 & 17 \\
\hline 13 & & & & & 8 & & & 8 & 8 \\
\hline \multirow[t]{4}{*}{17} & & & & & & & & 3 & 19 \\
\hline & & & & & & & & & 18 \\
\hline & & & & & & & & & 8 \\
\hline & & & & & & & & & 7 \\
\hline
\end{tabular}


Table 7.6. PSD for Sample 5476

\begin{tabular}{|c|c|c|c|c|c|c|c|c|c|}
\hline \multicolumn{10}{|c|}{ Data in $\mathrm{mm}(50 \times$ magnification $)$} \\
\hline \multicolumn{5}{|c|}{ Week 1} & \multicolumn{5}{|c|}{ Week 2} \\
\hline $5476-1$ & $5476-2$ & $5476-3$ & $5476-4$ & $5476-5$ & $5476-1$ & $5476-2$ & $5476-3$ & $5476-4$ & $5476-5$ \\
\hline 60.5 & 22.5 & 37 & 11 & 18 & 15 & 33 & 84 & 14 & 14 \\
\hline 35 & 17 & 59 & 37 & 22 & 13 & 16.5 & 30 & 20 & 25 \\
\hline 60 & 35 & 77 & 5.5 & 37 & 20 & 18 & 25 & 85 & 24 \\
\hline 73 & 8.5 & 65 & 8 & 20 & 8 & 55 & 68 & 58 & 19 \\
\hline 41 & 35 & 40 & 12 & 68 & 5 & 35 & 25 & 35 & 43 \\
\hline 38 & 15 & 29 & 15.5 & 78 & 33 & 40 & 35 & 70 & 25 \\
\hline 15 & 15 & 32 & 8 & 68 & 17 & 55 & 57 & 25 & 13 \\
\hline 25.5 & 41 & 72 & 10 & 44 & 43 & 78 & 12 & 11.2 & 28 \\
\hline 73 & 22 & 29 & 12.5 & 37 & 21 & 87 & 20 & 25 & 20 \\
\hline 29 & 7.5 & 32 & 21.5 & 81 & 42 & 34 & 24 & 60 & 45 \\
\hline 70 & 14.5 & 21 & 24 & 56 & 35 & 65 & 32 & 23 & 38 \\
\hline 72 & 13 & 56 & 31.5 & 20 & 5 & 24 & 11 & 11 & 6 \\
\hline 36 & 11 & 80 & 20 & 47 & 14 & & 17 & 7 & 48 \\
\hline 27 & 20.5 & 18 & 21.5 & 21 & 87 & & 34 & 17 & 80 \\
\hline 31 & 27.5 & 25 & 35.5 & 30 & 64 & & 65 & 32 & 30 \\
\hline 27 & 22 & 32 & 11 & 42.5 & 37 & & 9 & 34 & 22 \\
\hline 54 & 12 & 24 & 12 & 28 & 78 & & 13 & 31 & 57 \\
\hline 85 & 24 & 38 & 25 & 20 & 30 & & 30 & 33 & 48 \\
\hline 32 & 10 & 54 & 23 & 23 & 35 & & 64 & 64 & 35 \\
\hline 22 & 20 & 51 & 24 & 28 & 57 & & 11 & 10 & 18 \\
\hline 27 & & 31 & & 23 & 33 & & 34 & 8 & 20 \\
\hline 38 & & 21 & & 47 & & & 30 & 10 & 13 \\
\hline \multirow[t]{3}{*}{55} & & & & & & & 45 & 10 & 31 \\
\hline & & & & & & & 22 & 32 & \\
\hline & & & & & & & & 22 & \\
\hline
\end{tabular}





\subsection{Cr Caustic and Oxidative Leaching Tests}

Different chromium compounds leached differently, and the chromium oxyhydroxide slurry leached during the caustic leaching step of the pretreatment process, therefore not leaving enough chromium compound for the oxidative leaching step. Therefore, several different chromium compounds were examined to determine their caustic and oxidative leaching behavior to determine the best fit for the PEP simulant. These tests are discussed in this section.

The chromium oxyhydroxide slurry was eventually chosen for use in the PEP simulant. The chromium oxyhydroxide slurry (Cr-simulant) was procured separately as precipitated $\mathrm{CrOOH}$ slurry to be added before the oxidative leaching operation in the PEP. The production of this Cr-simulant was to be scaled up based on the initial trial batch prepared in December 2007. The scale up was to be achieved by initial production of about $15 \mathrm{~kg}$ of $\mathrm{Cr}(1 / 3$ scale of the production batch) to verify the proposed methods to concentrate and wash the precipitate $\mathrm{CrOOH}$ solids. Therefore, it was necessary to establish that the Cr-simulant produced both in the small batch $(15 \mathrm{~kg}$ of $\mathrm{Cr})$ and the production batch $(57 \mathrm{~kg}$ of $\mathrm{Cr}$ ) exhibits similar or better leaching behavior than the initial trial batch from 2007 that used in the CUF tests during caustic and oxidative leaching operations. The objective of these tests was to provide data showing that the Cr-simulant from the larger vendor-produced batches exhibited similar or improved leaching behavior than the initial smaller trial batch during caustic and oxidative leaching operations. WTP staff were responsible for interpreting these data and for assessing the impact of the data on planned PEP operations. As such, the data are presented herein, but there is no analysis provided.

\subsection{Chromium Characterization Leaching}

The objective of these tests was to characterize the dissolution of the different chromium compounds obtained from different vendors during caustic leaching at $100^{\circ} \mathrm{C}$ and oxidative leaching at $25^{\circ} \mathrm{C}$ with a stirring rate of $\sim 120 \mathrm{rpm}$. Table 8.1 identifies the manufacturer and chromium compound used in this testing. Appendix B provides the Statement of Work provided to the manufacturers (Noah Chemical and VWR) to produce the chromium hydroxide compounds for this testing. The chromium oxide obtained from Baker was laboratory-grade powder CAS No. 1308-38-9 (Lot \# E41599).

These tests were performed by placing $593 \mathrm{~g}$ of $5 \mathrm{M} \mathrm{NaOH}$ in the test vessel. The lid was attached, and the stirring was begun at $120 \mathrm{rpm}$. It was then heated to $100^{\circ} \mathrm{C}$. An initial sample was removed when it reached temperature. Then the required amount of the chromium compound was added to the vessel, and the time was recorded as zero. The actual amounts of components added are shown in Table 8.2. Samples were removed at 4 and 8 hours and filtered through a $0.45-\mu \mathrm{m}$ syringe filter. After caustic leaching at $100^{\circ} \mathrm{C}$, the batch was cooled to room temperature, and then $1 \mathrm{M} \mathrm{NaMnO}_{4}$ was added to the slurries (see Table 8.2 for amount), and they were oxidatively leached for 6 hours at room temperature $\left(\sim 23^{\circ} \mathrm{C}\right)$. Samples were taken at $1,2,4$, and 6 hours and filtered through a $0.45-\mu \mathrm{m}$ syringe filter. The supernate was analyzed for $\mathrm{CrO}_{4}{ }^{-}$by ultraviolet-visible light (UV-Vis) spectra, and the results are shown in Table 8.3 and Table 8.4. 
Table 8.1. Chromium Compound Identification

\begin{tabular}{ccc}
\hline Internal Compound ID & Compound & Manufacturer \\
\hline $\mathrm{C} 1$ & $\mathrm{Cr}(\mathrm{OH})_{3}$ & VWR \\
$\mathrm{C} 2$ & $\mathrm{CrOOH}$ & Noah Chemical \\
$\mathrm{C} 3$ & $\mathrm{Cr}(\mathrm{OH})_{3}$ & Noah Chemical \\
$\mathrm{C} 4$ & $\mathrm{CrOOH}$ & VWR \\
$\mathrm{C} 5$ & $\mathrm{Cr}_{2} \mathrm{O}_{3}$ & Baker Chemical \\
$\mathrm{C} 6$ & $\mathrm{CrOOH}$ & VWR \\
$\mathrm{C} 7$ & $\mathrm{CrOOH}$ & Noah Chemical \\
\hline
\end{tabular}

Table 8.2. Amounts of Components Used in Tests

\begin{tabular}{|c|c|c|c|}
\hline Internal Compound ID & $5 \mathrm{M} \mathrm{NaOH}(\mathrm{g})$ & Cr Compound (g) & $1 \mathrm{M} \mathrm{NaMnO}_{4}(\mathrm{~mL})$ \\
\hline $\mathrm{C} 1$ & 593.00 & 73.44 & 321 \\
\hline $\mathrm{C} 2$ & 593.01 & 40.89 & 305 \\
\hline $\mathrm{C} 3$ & 593.02 & 73.44 & 321 \\
\hline $\mathrm{C} 4$ & 593.01 & 40.89 & --- (a) \\
\hline $\mathrm{C} 5$ & 593.02 & 21.67 & 285 \\
\hline C6 & $---(b)$ & $---(b)$ & $--{ }^{\text {(a) }}$ \\
\hline C7 & --. (b) & --. (b) & --- (a) \\
\hline
\end{tabular}

(a) An oxidative leach was not performed on this one since it had been run in the crossflow ultrafiltration system (CUF) already.

(b) These tests were performed with the complete simulant matrix for caustic leaching only.

Determining the chromate concentration in the sample for C6 by ultraviolet visible (UV-Vis) was complicated by the interference of nitrate and especially nitrite in the sample. Therefore, the data were treated in two ways. One method was to assume that all of the $372-\mathrm{nm}$ absorbance was due to chromate, essentially ignoring the contribution due to nitrite. This gives a maximum estimated value for chromate (the middle column in Table 8.3.a). The other method was to assume that the 0 time had no chromate contribution to the 372-nm absorbance, and assign any 372-nm increase in later time measurements to chromate, essentially using the time 0 absorbance as a blank (the right column in Table 8.3.b).

Table 8.3.a. Cr Caustic Leaching Results from the Chromium Characterization Tests

\begin{tabular}{ccccc}
\hline $\begin{array}{c}\text { Time } \\
(\mathrm{hr})\end{array}$ & Sample ID & Test Description & $\begin{array}{c}{\left[\mathrm{CrO}_{4}{ }^{2-}\right], \mathrm{M},} \\
<\text { estimate }\end{array}$ & $\begin{array}{c}{\left[\mathrm{CrO}_{4}{ }^{2-}\right], \mathrm{M},} \\
\text { > estimate }\end{array}$ \\
\hline 0 & CL-C6-0 & Caustic leach of compound C6 & $1.88 \mathrm{E}-03$ & $0.00 \mathrm{E}+00$ \\
1 & CL-C6-1 & Caustic leach of compound C6 & $2.27 \mathrm{E}-03$ & $3.97 \mathrm{E}-04$ \\
2 & CL-C6-2 & Caustic leach of compound C6 & $2.47 \mathrm{E}-03$ & $5.94 \mathrm{E}-04$ \\
4 & CL-C6-4 & Caustic leach of compound C6 & $2.83 \mathrm{E}-03$ & $9.55 \mathrm{E}-04$ \\
8 & CL-C6-8 & Caustic leach of compound C6 & $3.11 \mathrm{E}-03$ & $1.24 \mathrm{E}-03$ \\
24 & CL-C6-24 & Caustic leach of compound C6 & $4.29 \mathrm{E}-03$ & $2.41 \mathrm{E}-03$ \\
\hline
\end{tabular}


Table 8.3.b (cont)

\begin{tabular}{|c|c|c|c|c|c|c|}
\hline $\begin{array}{l}\text { Time } \\
\text { (hr) }\end{array}$ & $\begin{array}{c}\mathrm{CL}-\mathrm{C} 1\left[\mathrm{CrO}_{4}{ }^{2-}\right] \\
\mathrm{M}\end{array}$ & $\begin{array}{c}\mathrm{CL}-\mathrm{C} 2\left[\mathrm{CrO}_{4}{ }^{2-}\right] \\
\mathrm{M}\end{array}$ & $\begin{array}{c}\mathrm{CL}-\mathrm{C} 3\left[\mathrm{CrO}_{4}{ }^{2-}\right] \\
\mathrm{M}\end{array}$ & $\begin{array}{c}\mathrm{CL}-\mathrm{C} 4\left[\mathrm{CrO}_{4}{ }^{2-}\right], \\
\mathrm{M}\end{array}$ & $\begin{array}{c}\text { CL-C5 }\left[\mathrm{CrO}_{4}{ }^{2-}\right], \\
\mathrm{M}\end{array}$ & $\begin{array}{c}\mathrm{CL}-\mathrm{C} 7\left[\mathrm{CrO}_{4}{ }^{2-}\right], \\
\mathrm{M}\end{array}$ \\
\hline & $\begin{array}{l}\text { Caustic leach of } \\
\text { compound } \mathrm{C} 1\end{array}$ & $\begin{array}{c}\text { Caustic leach of } \\
\text { compound C2 }\end{array}$ & $\begin{array}{c}\text { Caustic leach of } \\
\text { compound C3 }\end{array}$ & $\begin{array}{c}\text { Caustic leach of } \\
\text { compound C4 }\end{array}$ & $\begin{array}{c}\text { Caustic leach of } \\
\text { compound C5 }\end{array}$ & $\begin{array}{c}\text { Caustic leach of } \\
\text { compound C7 }\end{array}$ \\
\hline 4 & 7.81E-04 & 9.91E-04 & $4.84 \mathrm{E}-04$ & $6.81 \mathrm{E}-04$ & $1.29 \mathrm{E}-03$ & $8.10 \mathrm{E}-04$ \\
\hline 8 & $1.34 \mathrm{E}-03$ & $1.44 \mathrm{E}-03$ & $8.83 \mathrm{E}-04$ & $1.20 \mathrm{E}-03$ & $1.91 \mathrm{E}-03$ & $1.00 \mathrm{E}-03$ \\
\hline
\end{tabular}

Table 8.4. Cr Oxidative Leaching Results from the Chromium Characterization Tests

\begin{tabular}{ccccc}
\hline $\begin{array}{c}\text { Time } \\
(\mathrm{hr})\end{array}$ & $\begin{array}{c}\text { OL-C1 }\left[\mathrm{CrO}_{4}{ }^{2-}\right], \\
\mathrm{M}\end{array}$ & $\begin{array}{c}\text { OL-C2 }\left[\mathrm{CrO}_{4}{ }^{2-}\right], \\
\mathrm{M}\end{array}$ & $\begin{array}{c}\text { OL-C3 }\left[\mathrm{CrO}_{4}{ }^{2-}\right], \\
\mathrm{M}\end{array}$ & $\begin{array}{c}\text { OL-C5 }\left[\mathrm{CrO}_{4}{ }^{2-}\right], \\
\mathrm{M}\end{array}$ \\
\hline & $\begin{array}{c}\text { Oxidative leach } \\
\text { of compound C1 }\end{array}$ & $\begin{array}{c}\text { Oxidative leach } \\
\text { of compound C2 }\end{array}$ & $\begin{array}{c}\text { Oxidative leach of } \\
\text { compound C3 }\end{array}$ & $\begin{array}{c}\text { Oxidative leach of } \\
\text { compound C5 }\end{array}$ \\
\hline 1 & $3.31 \mathrm{E}-01$ & $3.45 \mathrm{E}-01$ & $3.79 \mathrm{E}-01$ & $1.04 \mathrm{E}-01$ \\
2 & $3.63 \mathrm{E}-01$ & $3.62 \mathrm{E}-01$ & $3.85 \mathrm{E}-01$ & $1.29 \mathrm{E}-01$ \\
4 & $3.43 \mathrm{E}-01$ & $3.70 \mathrm{E}-01$ & $3.79 \mathrm{E}-01$ & $1.47 \mathrm{E}-01$ \\
6 & $3.40 \mathrm{E}-01$ & $3.60 \mathrm{E}-01$ & $3.95 \mathrm{E}-01$ & $1.55 \mathrm{E}-01$ \\
\hline
\end{tabular}

\subsection{Full Matrix Chromium Leaching Tests}

The objective of these tests was to characterize the dissolution of the different chromium compounds obtained from the different vendors in the full simulant matrix during caustic leaching at $100^{\circ} \mathrm{C}$ with a stirring rate of $\sim 120 \mathrm{rpm}$. Table 8.5 identifies the test identification, the chromium compound, and vendor for each test performed. Appendix B provides the Statement of Work provided to the manufacturers (Noah Chemical and VWR) to produce the chromium hydroxide compounds for this testing. The chromium oxide obtained from Baker was laboratory-grade powder CAS No. 1308-38-9 (Lot \# E41599).

Table 8.5. Chromium Compound Identification for Full Matrix Testing

\begin{tabular}{ccc}
\hline Internal Compound ID & Compound & Manufacturer \\
\hline FMC1 & $\mathrm{Cr}(\mathrm{OH})_{3}$ & VWR \\
FMC2 & $\mathrm{CrOOH}$ & Noah Chemical \\
FMC3 & $\mathrm{Cr}(\mathrm{OH})_{3}$ & Noah Chemical \\
FMC4 & $\mathrm{CrOOH} \mathrm{Slurry}$ & Noah Chemical \\
FMC5 & $\mathrm{Cr}_{2} \mathrm{O}_{3}$ & Baker Chemical \\
\hline
\end{tabular}

These tests were performed by placing $264 \mathrm{~g}$ of iron-rich sludge slurry in the test vessel. Then $6.5 \mathrm{~g}$ of sodium oxalate, $57.2 \mathrm{~g}$ of boehmite, $57.2 \mathrm{~g}$ of gibbsite, and $14 \mathrm{~g}$ of supernate, $200.3 \mathrm{~g}$ of $19 \mathrm{M} \mathrm{NaOH}$, and $199 \mathrm{~g}$ of DI water were added to the test vessel. The lid was attached, and the stirring began at $120 \mathrm{rpm}$. It was then heated to $100^{\circ} \mathrm{C}$. An initial sample was removed when it reached temperature. 
Approximately $1.9 \mathrm{~g}$ of the chromium compound was added to the vessel, and the time was recorded as zero. The actual amounts of components added are shown in Table 8.6. Samples (FMC) were then removed at $1,2,4,8$, and 24 hours and filtered through a $0.45-\mu \mathrm{m}$ syringe filter. The supernate was analyzed for Al, Cr, and Na by ICP-AES at SWRI, and the results are shown in Table 8.7 through Table 8.9. Then $1 \mathrm{M} \mathrm{NaMnO}_{4}$ was added to the slurries (see Table 8.6 for amount), and they were oxidatively leached for 6 hours at room temperature $\left(\sim 23^{\circ} \mathrm{C}\right)$. Samples (FMO) were taken at 1, 2, 4, and 6 hours and filtered through a $0.45-\mu \mathrm{m}$ syringe filter. The supernate was analyzed for Cr by UV-Vis, and the results are shown in Table 8.10.

Table 8.6. Components Added to the Full Matrix Chromium Leaching Tests

\begin{tabular}{|c|c|c|c|c|c|c|c|c|c|}
\hline $\begin{array}{l}\text { Test } \\
\text { ID }\end{array}$ & $\begin{array}{c}\text { Fe- } \\
\text { Rich } \\
\text { Sludge } \\
\text { (g) }\end{array}$ & $\begin{array}{l}\text { Sodium } \\
\text { Oxalate } \\
\text { (g) }\end{array}$ & $\begin{array}{c}\text { Boehmite } \\
\text { (g) }\end{array}$ & $\begin{array}{c}\text { Gibbsite } \\
\text { (g) }\end{array}$ & $\begin{array}{l}\text { Supernate } \\
\text { (g) }\end{array}$ & $\begin{array}{c}19 \mathrm{M} \\
\mathrm{NaOH} \\
(\mathrm{g})\end{array}$ & $\begin{array}{c}\text { DI } \\
\text { Water } \\
(\mathrm{g})\end{array}$ & $\begin{array}{c}\mathrm{Cr} \\
\text { Compound } \\
\text { (g) }\end{array}$ & $\begin{array}{c}1 \mathrm{M} \\
\mathrm{NaMnO}_{4} \\
(\mathrm{~mL})\end{array}$ \\
\hline$\overline{\text { FMC1 }}$ & 264.01 & 6.50 & 57.20 & 57.20 & 14.00 & 200.28 & 198.90 & 1.93 & 19 \\
\hline FMC2 & 264.02 & 6.50 & 57.20 & 57.20 & 14.01 & 200.28 & 198.92 & 1.93 & 23 \\
\hline FMC3 & 264.02 & 6.50 & 57.20 & 57.20 & 14.01 & 200.28 & 198.92 & 1.93 & 19 \\
\hline FMC4 & 264.03 & 6.50 & 57.20 & 57.20 & 14.01 & 200.28 & 198.90 & 1.94 & 23 \\
\hline FMC5 & 264.01 & 6.50 & 57.20 & 57.20 & 14.00 & 200.43 & 198.97 & 1.93 & 25 \\
\hline
\end{tabular}

Table 8.7. Al Results from the Full Matrix Chromium Caustic Leaching Tests

\begin{tabular}{cccccc}
\hline Time $(\mathrm{hr})$ & $\begin{array}{c}\text { FMC1 } \\
(\mathrm{mg} \mathrm{Al} / \mathrm{kg} \mathrm{soln})\end{array}$ & $\begin{array}{c}\text { FMC2 } \\
(\mathrm{mg} \mathrm{Al} / \mathrm{kg} \mathrm{soln})\end{array}$ & $\begin{array}{c}\text { FMC3 } \\
(\mathrm{mg} \mathrm{Al} / \mathrm{kg} \mathrm{soln})\end{array}$ & $\begin{array}{c}\text { FMC4 } \\
(\mathrm{mg} \mathrm{Al} / \mathrm{kg} \mathrm{soln})\end{array}$ & $\begin{array}{c}\text { FMC5 } \\
(\mathrm{mg} \mathrm{Al} / \mathrm{kg} \mathrm{soln})\end{array}$ \\
\hline 0 & 27,000 & 27,000 & 26,900 & 27,600 & 27,200 \\
1 & 26,900 & 26,100 & 26,100 & 27,400 & 27,000 \\
2 & 28,200 & 27,800 & 26,400 & 26,800 & 27,200 \\
4 & 26,800 & 26,900 & 26,200 & 27,500 & 27,400 \\
8 & 27,100 & 27,800 & 26,400 & 28,700 & 28,800 \\
24 & 30,000 & 27,900 & 26,200 & 29,500 & 30,700 \\
\hline
\end{tabular}

Table 8.8. Cr Results from the Full Matrix Chromium Caustic Leaching Tests

\begin{tabular}{cccccc}
\hline Time $(\mathrm{hr})$ & $\begin{array}{c}\text { FMC1 } \\
(\mathrm{mg} \mathrm{Cr} / \mathrm{kg} \mathrm{soln})\end{array}$ & $\begin{array}{c}\text { FMC2 } \\
(\mathrm{mg} \mathrm{Cr} / \mathrm{kg} \mathrm{soln})\end{array}$ & $\begin{array}{c}\text { FMC3 } \\
(\mathrm{mg} \mathrm{Cr} / \mathrm{kg} \mathrm{soln})\end{array}$ & $\begin{array}{c}\text { FMC4 } \\
(\mathrm{mg} \mathrm{Cr} / \mathrm{kg} \mathrm{soln})\end{array}$ & $\begin{array}{c}\text { FMC5 } \\
(\mathrm{mg} \mathrm{Cr} / \mathrm{kg} \mathrm{soln})\end{array}$ \\
\hline 0 & 79.3 & 83.2 & 82.6 & 84.3 & 76.0 \\
1 & 282 & 166 & 589 & 117 & 89.6 \\
2 & 318 & 214 & 646 & 128 & 95.2 \\
4 & 351 & 269 & 693 & 140 & 102 \\
8 & 403 & 360 & 749 & 151 & 113 \\
24 & 515 & 584 & 820 & 172 & 135 \\
\hline
\end{tabular}


Table 8.9. Na Results from the Full Matrix Chromium Caustic Leaching Tests

\begin{tabular}{cccccc}
\hline Time $(\mathrm{hr})$ & $\begin{array}{c}\text { FMC1 } \\
(\mathrm{mg} \mathrm{Na} / \mathrm{kg} \text { soln })\end{array}$ & $\begin{array}{c}\text { FMC2 } \\
(\mathrm{mg} \mathrm{Na} / \mathrm{kg} \mathrm{soln})\end{array}$ & $\begin{array}{c}\text { FMC3 } \\
(\mathrm{mg} \mathrm{Na} / \mathrm{kg} \text { soln })\end{array}$ & $\begin{array}{c}\text { FMC4 } \\
(\mathrm{mg} \mathrm{Na} / \mathrm{kg} \mathrm{soln})\end{array}$ & $\begin{array}{c}\text { FMC5 } \\
(\mathrm{mg} \mathrm{Na} / \mathrm{kg} \text { soln })\end{array}$ \\
\hline 0 & 85,100 & 85,900 & 84,600 & 84,800 & 84,500 \\
1 & 85,200 & 84,900 & 85,000 & 84,200 & 84,700 \\
2 & 86,000 & 87,800 & 85,700 & 85,100 & 85,800 \\
4 & 86,500 & 86,200 & 86,500 & 87,600 & 88,100 \\
8 & 88,400 & 87,500 & 87,800 & 89,300 & 90,000 \\
24 & 105,000 & 91,500 & 86,500 & 93,700 & 97,300 \\
\hline
\end{tabular}

Table 8.10. Cr Results from the Full Matrix Chromium Oxidative Leaching Tests

\begin{tabular}{cccccc}
\hline Time (hr) & $\mathrm{FMO} 1\left[\mathrm{CrO}_{4}{ }^{2-}\right] \mathrm{M}$ & $\mathrm{FMO} 2\left[\mathrm{CrO}_{4}{ }^{2-}\right] \mathrm{M}$ & $\mathrm{FMO} 3\left[\mathrm{CrO}_{4}{ }^{2-}\right] \mathrm{M}$ & $\mathrm{FMO} 4\left[\mathrm{CrO}_{4}{ }^{2-}\right] \mathrm{M}$ FMO5 $\left[\mathrm{CrO}{ }_{4}{ }^{2-}\right] \mathrm{M}$ \\
\hline 0 & $1.25 \mathrm{E}-02$ & $1.67 \mathrm{E}-02$ & $2.21 \mathrm{E}-02$ & $4.61 \mathrm{E}-03$ & $3.51 \mathrm{E}-03$ \\
1 & $1.70 \mathrm{E}-02$ & $2.73 \mathrm{E}-02$ & $2.25 \mathrm{E}-02$ & $4.78 \mathrm{E}-03$ & $5.89 \mathrm{E}-03$ \\
2 & $1.69 \mathrm{E}-02$ & $2.88 \mathrm{E}-02$ & $2.23 \mathrm{E}-02$ & $4.76 \mathrm{E}-03$ & $7.44 \mathrm{E}-03$ \\
4 & $1.72 \mathrm{E}-02$ & $3.13 \mathrm{E}-02$ & $2.22 \mathrm{E}-02$ & $4.74 \mathrm{E}-03$ & $1.00 \mathrm{E}-02$ \\
6 & $1.74 \mathrm{E}-02$ & $3.27 \mathrm{E}-02$ & $2.22 \mathrm{E}-02$ & $4.75 \mathrm{E}-03$ & $1.15 \mathrm{E}-02$ \\
\hline
\end{tabular}

\subsection{Chromium Oxide Leaching Tests}

The objective of these tests was to characterize the dissolution of different chromium oxide compounds obtained from different vendors during caustic leaching at $100^{\circ} \mathrm{C}$ and oxidative leaching at $25^{\circ} \mathrm{C}$ with a stirring rate of $\sim 120 \mathrm{rpm}$. Table 8.11 identifies the manufacturer and test ID of the different compounds tested. These were technical grade chromium oxide compounds that were purchased as-is from the manufacturer.

Table 8.11. Chromium Oxide Identification

\begin{tabular}{cc}
\hline Test ID & Manufacturer \\
\hline $\mathrm{CO} 1$ & Sigma-Aldrich \\
$\mathrm{CO} 2$ & Fisher Scientific \\
$\mathrm{CO} 3$ & Alfa AESAR \\
$\mathrm{CO} 4$ & Mallincrodt Baker \\
& American Elements \\
$\mathrm{CO} 5$ & (CR-OX-02-NP) \\
& American Elements \\
$\mathrm{CO} 6$ & (CR-OX-02) \\
\hline
\end{tabular}

These tests were performed by placing $264 \mathrm{~g}$ of iron-rich sludge slurry in the test vessel. Then $6.5 \mathrm{~g}$ of sodium oxalate, $57.2 \mathrm{~g}$ of boehmite, $57.2 \mathrm{~g}$ of gibbsite, and $14 \mathrm{~g}$ of supernate, $200.3 \mathrm{~g}$ of $19 \mathrm{M} \mathrm{NaOH}$, and $199 \mathrm{~g}$ of DI water were added to the test vessel. The lid was attached, and the stirring was begun at $120 \mathrm{rpm}$. It was then heated to $100^{\circ} \mathrm{C}$. An initial sample was removed when it reached temperature. Approximately $1.9 \mathrm{~g}$ of the chromium compound was added to the vessel, and the time was recorded as 
zero. The actual amounts of components added are shown in Table 8.12. Samples were then removed at $1,2,4,8$, and 24 hours and filtered through a $0.45-\mu \mathrm{m}$ syringe filter. The supernate was analyzed for Al, $\mathrm{Cr}$, and $\mathrm{Na}$ by ICP-AES at SWRI, and the results are shown in Table 8.13 through Table 8.15. Then $1 \mathrm{M}$ $\mathrm{NaMnO}_{4}$ was added to the slurries (see Table 8.12 for amount), and they were oxidatively leached for 6 hours at room temperature $\left(\sim 23^{\circ} \mathrm{C}\right)$. Samples were taken at $1,2,4$, and 6 hours and filtered through a $0.45-\mu \mathrm{m}$ syringe filter. The supernate was analyzed for Al, $\mathrm{Cr}$, and $\mathrm{Na}$ by ICP-AES, and the results are shown in Table 8.16 through Table 8.18. Test ID CL indicates caustic leaching, and OL indicates oxidative leaching.

Table 8.12. Components Added to the Chromium Oxide Leaching Tests

\begin{tabular}{|c|c|c|c|c|c|c|c|c|c|}
\hline $\begin{array}{l}\text { Test } \\
\text { ID }\end{array}$ & $\begin{array}{c}\text { Fe- } \\
\text { Rich } \\
\text { Sludge } \\
(\mathrm{g}) \\
\end{array}$ & $\begin{array}{c}\text { Sodium } \\
\text { Oxalate } \\
(\mathrm{g})\end{array}$ & $\begin{array}{c}\text { Boehmite } \\
(\mathrm{g})\end{array}$ & $\begin{array}{c}\text { Gibbsite } \\
(\mathrm{g})\end{array}$ & $\begin{array}{c}\text { Supernate } \\
(\mathrm{g})\end{array}$ & $\begin{array}{c}19 \mathrm{M} \\
\mathrm{NaOH} \\
(\mathrm{g}) \\
\end{array}$ & $\begin{array}{c}\text { DI } \\
\text { Water } \\
(\mathrm{g}) \\
\end{array}$ & $\begin{array}{c}\mathrm{Cr} \\
\text { Compound } \\
(\mathrm{g})\end{array}$ & $\begin{array}{c}1 \mathrm{M} \\
\mathrm{NaMnO}_{4} \\
(\mathrm{~mL})\end{array}$ \\
\hline $\mathrm{CO} 1$ & 264.0 & 6.5 & 57.2 & 57.2 & 14.0 & 200.3 & 198.9 & 1.93 & 25 \\
\hline $\mathrm{CO} 2$ & 264.0 & 6.5 & 57.2 & 57.2 & 14.0 & 200.3 & 198.9 & 1.92 & 25 \\
\hline $\mathrm{CO} 3$ & 264.0 & 6.5 & 57.2 & 57.2 & 14.0 & 200.3 & 198.9 & 1.93 & 25 \\
\hline $\mathrm{CO} 4$ & 264.00 & 6.50 & 57.20 & 57.20 & 14.01 & 200.28 & 198.91 & 1.93 & 25 \\
\hline $\mathrm{CO} 5$ & 264.02 & 6.50 & 57.20 & 57.20 & 14.05 & 200.27 & 198.89 & 1.93 & 25 \\
\hline $\mathrm{CO} 6$ & 264.01 & 6.50 & 57.20 & 57.21 & 14.03 & 200.26 & 198.91 & 1.93 & 25 \\
\hline
\end{tabular}

Table 8.13. Al Results from the Chromium Oxide Caustic Leaching Tests

\begin{tabular}{ccccccc}
\hline $\begin{array}{c}\text { Time } \\
(\mathrm{hr})\end{array}$ & $\begin{array}{c}\text { CL-CO1 (mg } \\
\text { Al/kg soln) }\end{array}$ & $\begin{array}{c}\text { CL-CO2 (mg } \\
\text { Al/kg soln) }\end{array}$ & $\begin{array}{c}\text { CL-CO3 (mg } \\
\text { Al/kg soln) }\end{array}$ & $\begin{array}{c}\text { CL-CO4 (mg } \\
\text { Al/kg soln) }\end{array}$ & $\begin{array}{c}\text { CL-CO5 (mg } \\
\text { Al/kg soln) }\end{array}$ & $\begin{array}{c}\text { CL-CO6 (mg } \\
\text { Al/kg soln) }\end{array}$ \\
\hline 0 & 26,300 & 25,500 & 26,500 & 25,800 & 26,500 & 25,300 \\
1 & 25,500 & 26,000 & 26,700 & 24,800 & 25,000 & 24,600 \\
2 & 27,300 & 26,000 & 26,500 & 25,500 & 25,000 & 25,100 \\
4 & 26,000 & 26,200 & 26,100 & 25,100 & 26,200 & 25,100 \\
8 & 26,800 & 26,600 & 27,400 & 26,400 & 27,100 & 27,500 \\
24 & 26,400 & 28,000 & 29,100 & No sample & No sample & 24,700 \\
\hline
\end{tabular}

Table 8.14. Cr Results from the Chromium Oxide Caustic Leaching Tests

\begin{tabular}{ccccccc}
\hline $\begin{array}{c}\text { Time } \\
(\mathrm{hr})\end{array}$ & $\begin{array}{c}\text { CL-CO1 }(\mathrm{mg} \\
\text { Cr/kg soln) }\end{array}$ & $\begin{array}{c}\text { CL-CO2 }(\mathrm{mg} \\
\text { Cr/kg soln) }\end{array}$ & $\begin{array}{c}\text { CL-CO3 (mg } \\
\text { Cr/kg soln) }\end{array}$ & $\begin{array}{c}\text { CL-CO4 (mg } \\
\text { Cr/kg soln) }\end{array}$ & $\begin{array}{c}\text { CL-CO5 (mg } \\
\text { Cr/kg soln) }\end{array}$ & $\begin{array}{c}\text { CL-CO6 (mg } \\
\text { Cr/kg soln) }\end{array}$ \\
\hline 0 & 77.0 & 77.3 & 77.3 & 77.2 & 82.2 & 82.3 \\
1 & 92.1 & 88.9 & 92.0 & 84.7 & 87.1 & 89.8 \\
2 & 101 & 94.2 & 94.9 & 88.7 & 90.6 & 91.5 \\
4 & 104 & 99.1 & 99.8 & 92.3 & 96.5 & 94.2 \\
8 & 113 & 107 & 108 & 96.7 & 101 & 101 \\
24 & 125 & 123 & 124 & No sample & No sample & 104 \\
\hline
\end{tabular}


Table 8.15. Na Results from the Chromium Oxide Caustic Leaching Tests

\begin{tabular}{ccccccc}
\hline $\begin{array}{c}\text { Time } \\
\text { (hr) }\end{array}$ & $\begin{array}{c}\text { CL-CO1 (mg } \\
\text { Na/kg soln) }\end{array}$ & $\begin{array}{c}\text { CL-CO2 (mg } \\
\text { Na/kg soln) }\end{array}$ & $\begin{array}{c}\text { CL-CO3 (mg } \\
\text { Na/kg soln) }\end{array}$ & $\begin{array}{c}\text { CL-CO4 (mg } \\
\text { Na/kg soln) }\end{array}$ & $\begin{array}{c}\text { CL-CO5 (mg } \\
\text { Na/kg soln) }\end{array}$ & $\begin{array}{c}\text { CL-CO6 (mg } \\
\text { Na/kg soln) }\end{array}$ \\
\hline 0 & 84,200 & 85,200 & 85,200 & 83,300 & 84,200 & 85,100 \\
1 & 85,300 & 86,300 & 86,800 & 83,100 & 83,500 & 83,500 \\
2 & 87,500 & 86,500 & 86,300 & 83,300 & 85,200 & 84,400 \\
4 & 86,300 & 87,000 & 88,000 & 82,200 & 84,400 & 84,100 \\
8 & 87,400 & 89,200 & 89,600 & 82,800 & 86,400 & 86,400 \\
24 & 87,500 & 94,900 & 93,600 & No sample & No sample & 83,100 \\
\hline
\end{tabular}

Table 8.16. Al Results from the Chromium Oxide Oxidative Leaching Tests

\begin{tabular}{ccccccc}
\hline $\begin{array}{c}\text { Time } \\
(\mathrm{hr})\end{array}$ & $\begin{array}{c}\text { OL-CO1 }(\mathrm{mg} \\
\text { Al/kg soln) }\end{array}$ & $\begin{array}{c}\text { OL-CO2 } \\
(\mathrm{mg} \mathrm{A1} / \mathrm{kg} \\
\text { soln })\end{array}$ & $\begin{array}{c}\text { OL-CO3 } \\
(\mathrm{mg} \mathrm{Al} / \mathrm{kg} \\
\text { soln })\end{array}$ & $\begin{array}{c}\text { OL-CO4 } \\
(\mathrm{mg} \mathrm{Al} / \mathrm{kg} \\
\text { soln })\end{array}$ & $\begin{array}{c}\text { OL-CO5 } \\
(\mathrm{mg} \mathrm{Al} / \mathrm{kg} \\
\text { soln })\end{array}$ & $\begin{array}{c}\text { OL-CO6 } \\
(\mathrm{mg} \mathrm{Al} / \mathrm{kg} \\
\text { soln) }\end{array}$ \\
\hline 0 & 28,100 & 30,200 & 29,700 & 26,900 & 28,900 & 28,600 \\
1 & 26,300 & 28,400 & 28,900 & 24,400 & 27,100 & 28,100 \\
2 & 27,300 & 27,900 & 28,200 & 24,900 & 27,100 & 27,100 \\
4 & 26,000 & 28,300 & 28,600 & 24,300 & 28,000 & 26,800 \\
6 & 26,200 & 28,100 & 28,600 & 25,700 & 29,200 & 26,700 \\
\hline
\end{tabular}

Table 8.17. Cr Results from the Chromium Oxide Oxidative Leaching Tests

\begin{tabular}{ccccccc}
\hline $\begin{array}{c}\text { Time } \\
(\mathrm{hr})\end{array}$ & $\begin{array}{c}\text { OL-CO1 }(\mathrm{mg} \\
\text { Cr/kg soln) }\end{array}$ & $\begin{array}{c}\text { OL-CO2 }(\mathrm{mg} \\
\text { Cr/kg soln) }\end{array}$ & $\begin{array}{c}\text { OL-CO3 } \\
(\mathrm{mg} \mathrm{Cr} / \mathrm{kg} \\
\text { soln) }\end{array}$ & $\begin{array}{c}\text { OL-CO4 } \\
(\mathrm{mg} \mathrm{Cr} / \mathrm{kg} \\
\text { soln })\end{array}$ & $\begin{array}{c}\text { OL-CO5 } \\
(\mathrm{mg} \mathrm{Cr} / \mathrm{kg} \\
\text { soln })\end{array}$ & $\begin{array}{c}\text { OL-CO6 } \\
(\mathrm{mg} \mathrm{Cr} / \mathrm{kg} \\
\text { soln })\end{array}$ \\
\hline 0 & 125 & 124 & 121 & 105 & 121 & 120 \\
1 & 146 & 133 & 127 & 106 & 126 & 120 \\
2 & 169 & 136 & 128 & 107 & 140 & 121 \\
4 & 190 & 151 & 133 & 110 & 162 & 124 \\
6 & 217 & 165 & 139 & 109 & 182 & 127 \\
\hline
\end{tabular}

Table 8.18. Na Results from the Chromium Oxide Oxidative Leaching Tests

\begin{tabular}{ccccccc}
\hline $\begin{array}{c}\text { Time } \\
(\mathrm{hr})\end{array}$ & $\begin{array}{c}\text { OL-CO1 }(\mathrm{mg} \\
\text { Na/kg soln) }\end{array}$ & $\begin{array}{c}\text { OL-CO2 }(\mathrm{mg} \\
\mathrm{Na} / \mathrm{kg} \mathrm{soln})\end{array}$ & $\begin{array}{c}\text { OL-CO3 } \\
(\mathrm{mg} \mathrm{Na} / \mathrm{kg} \\
\text { soln) }\end{array}$ & $\begin{array}{c}\text { OL-CO4 } \\
(\mathrm{mg} \mathrm{Na} / \mathrm{kg} \\
\text { soln })\end{array}$ & $\begin{array}{c}\text { OL-CO5 } \\
(\mathrm{mg} \mathrm{Na} / \mathrm{kg} \\
\text { soln) }\end{array}$ & $\begin{array}{c}\text { OL-CO6 (mg } \\
\mathrm{Na} / \mathrm{kg} \text { soln) }\end{array}$ \\
\hline 0 & 85,600 & 91,900 & 93,400 & 78,900 & 91,900 & 91,800 \\
1 & 83,300 & 87,800 & 90,500 & 79,500 & 89,100 & 88,200 \\
2 & 85,500 & 87,600 & 89,600 & 79,500 & 88,500 & 88,100 \\
4 & 82,800 & 88,900 & 90,700 & 79,200 & 88,300 & 88,200 \\
6 & 82,600 & 87,400 & 90,000 & 78,900 & 89,100 & 88,200 \\
\hline
\end{tabular}




\subsection{Chromium Oxidative Leach Investigation Tests}

The objective of these tests was to investigate the dissolution of different chromium compounds under different conditions during oxidative leaching at $25^{\circ} \mathrm{C}$ with a stirring rate of $\sim 120 \mathrm{rpm}$. Each chromium compound used is listed in Table 8.19. Appendix B provides the Statement of Work provided to the manufacturers to produce the chromium hydroxide compounds for this testing. The chromium oxide compounds were just technical grade that were purchased from the manufacturer's catalog.

These tests were performed in a variety of ways. The amounts used for each test are shown in Table 8.20. The first test (CLI1) was performed by mixing boehmite, gibbsite, sludge slurry, sodium oxalate, chromium oxide, supernate, DI water, and $19 \mathrm{M} \mathrm{NaOH}$ together in the test vessel. Then it was centrifuged and the supernate decanted. The remaining solids were washed three times with $0.01 \mathrm{M}$ $\mathrm{NaOH}$ and centrifuged and decanted each time. The washed slurry was placed back into the test vessel and $5 \mathrm{M} \mathrm{NaOH}$ and $1 \mathrm{M} \mathrm{NaMnO}_{4}$ were added. The test was run for 6 hours with samples taken at 1, 2, 4, and 6 hours.

The second test (CLI2) was performed by placing $500-\mathrm{mL}$ of $0.25 \mathrm{M} \mathrm{NaOH}$ in the test vessel and adding $1.93 \mathrm{~g} \mathrm{Cr}_{2} \mathrm{O}_{3}$. Then $25-\mathrm{mL}$ of $1 \mathrm{M} \mathrm{NaMnO}_{4}$ was added, and the test was run for 6 hours with samples taken at $1,2,4$, and 6 hours.

The rest of the tests (CLI4a, CLI4b, and CLI5) were performed the same way as the first one by mixing boehmite, gibbsite, sludge slurry, sodium oxalate, supernate, DI water, and $19 \mathrm{M} \mathrm{NaOH}$ together in the test vessel, but using different chromium sources. It was then heated to $100^{\circ} \mathrm{C}$. After reaching temperature, the chromium compound was added, and samples were removed at $0,1,2,4,8$, and 24 hours. The slurry was cooled, centrifuged, decanted, and washed three times with $0.01 \mathrm{M} \mathrm{NaOH}$, centrifuging and decanting each time. The washed slurry was placed back into the test vessel and $5 \mathrm{M}$ $\mathrm{NaOH}$ and $1 \mathrm{M} \mathrm{NaMnO}_{4}$ were added. The test was then run for 6 hours at room temperature $\left(\sim 23^{\circ} \mathrm{C}\right)$ with samples taken at 1,2, 4, and 6 hours. The supernate from both the caustic and oxidative leaches was analyzed for Cr by UV-Vis, and the results are shown in Table 8.21 and Table 8.22.

Table 8.19. Chromium Investigative Leach Test Compound Identification

\begin{tabular}{cc}
\hline Test ID & Manufacturer \\
\hline CLI1 & Sigma-Aldrich $\mathrm{Cr}_{2} \mathrm{O}_{3}$ \\
CLI2 & Sigma-Aldrich $\mathrm{Cr}_{2} \mathrm{O}_{3}$ \\
CLI4a & Noah CrOOH \\
CLI4b & VWR CrOOH \\
CLI5 & Sigma-Aldrich $\mathrm{Cr}_{2} \mathrm{O}_{3}$ \\
\hline
\end{tabular}


Table 8.20. Components Added to the Chromium Investigative Leach Tests

\begin{tabular}{|c|c|c|c|c|c|c|c|c|c|c|}
\hline $\begin{array}{l}\text { Test } \\
\text { ID }\end{array}$ & $\begin{array}{c}\text { Fe- } \\
\text { Rich } \\
\text { Sludge } \\
\text { (g) }\end{array}$ & $\begin{array}{c}\text { Sodium } \\
\text { Oxalate } \\
\text { (g) }\end{array}$ & $\begin{array}{c}\text { Boehmite } \\
(\mathrm{g})\end{array}$ & $\begin{array}{c}\text { Gibbsite } \\
(\mathrm{g})\end{array}$ & $\begin{array}{c}\text { Supernate } \\
\text { (g) }\end{array}$ & $\begin{array}{c}19 \mathrm{M} \\
\mathrm{NaOH} \\
(\mathrm{g})\end{array}$ & $\begin{array}{c}\text { DI } \\
\text { Water } \\
(\mathrm{g})\end{array}$ & $\begin{array}{c}\mathrm{Cr} \\
\text { Compound } \\
(\mathrm{g})\end{array}$ & $\mathrm{NaOH}$ & $\begin{array}{c}1 \mathrm{M} \\
\mathrm{NaMnO}_{4} \\
(\mathrm{~mL})\end{array}$ \\
\hline CLI1 & 264.01 & 6.50 & 57.20 & 57.20 & 13.99 & 200.28 & 198.92 & 1.93 & $\begin{array}{l}36.7 \mathrm{~g} \\
(5 \mathrm{M})\end{array}$ & 25 \\
\hline CLI2 & -- & -- & -- & -- & -- & -- & -- & 1.93 & $\begin{array}{c}500-\mathrm{mL} \\
(0.25 \\
\mathrm{M})\end{array}$ & 25 \\
\hline CLI4a & 247.33 & 15.60 & 54.10 & 54.10 & 17.01 & 200.26 & 198.91 & 12.51 & $\begin{array}{l}48.6 \mathrm{~g} \\
(5 \mathrm{M})\end{array}$ & 25 \\
\hline CLI4b & 247.30 & 15.60 & 54.10 & 54.10 & 17.01 & 200.27 & 198.89 & 12.51 & $\begin{array}{l}48.6 \mathrm{~g} \\
(5 \mathrm{M})\end{array}$ & 25 \\
\hline CLI5 & 247.31 & 15.60 & 54.10 & 54.12 & 17.00 & 200.27 & 198.91 & 1.93 & $\begin{array}{l}48.6 \mathrm{~g} \\
(5 \mathrm{M})\end{array}$ & 25 \\
\hline
\end{tabular}

Table 8.21. Cr Results from the Chromium Investigative Caustic Leach Tests

\begin{tabular}{cccc}
\hline $\begin{array}{c}\text { Time } \\
(\mathrm{hr})\end{array}$ & $\begin{array}{c}\mathrm{CLI} 4 \mathrm{a} \\
{\left[\mathrm{CrO}_{4}{ }^{2-}\right] \mathrm{M}}\end{array}$ & $\begin{array}{c}\text { CLI4b } \\
{\left[\mathrm{CrO}_{4}{ }^{2-}\right] \mathrm{M}}\end{array}$ & $\begin{array}{c}\mathrm{CLI} 5\left[\mathrm{CrO}_{4}{ }^{2-}\right] \\
\mathrm{M}\end{array}$ \\
\hline 0 & $1.78 \mathrm{E}-03$ & $1.87 \mathrm{E}-03$ & $1.89 \mathrm{E}-03$ \\
1 & $1.06 \mathrm{E}-02$ & $1.19 \mathrm{E}-02$ & $2.27 \mathrm{E}-03$ \\
2 & $1.38 \mathrm{E}-02$ & $1.51 \mathrm{E}-02$ & $2.37 \mathrm{E}-03$ \\
4 & $1.74 \mathrm{E}-02$ & $1.81 \mathrm{E}-02$ & $2.40 \mathrm{E}-03$ \\
8 & $2.04 \mathrm{E}-02$ & $2.06 \mathrm{E}-02$ & $2.56 \mathrm{E}-03$ \\
24 & $2.95 \mathrm{E}-02$ & $2.81 \mathrm{E}-02$ & $2.87 \mathrm{E}-03$ \\
\hline
\end{tabular}

Table 8.22. Cr Results from the Chromium Investigative Oxidative Leach Tests

\begin{tabular}{cccccc}
\hline $\begin{array}{c}\text { Time } \\
(\mathrm{hr})\end{array}$ & $\begin{array}{c}\mathrm{CLI} 1 \\
{\left[\mathrm{CrO}_{4}{ }^{2-}\right] \mathrm{M}}\end{array}$ & $\begin{array}{c}\mathrm{CLI} 2 \\
{\left[\mathrm{CrO}_{4}{ }^{2-}\right] \mathrm{M}}\end{array}$ & $\begin{array}{c}\text { CLI4a } \\
{\left[\mathrm{CrO}_{4}{ }^{2-}\right] \mathrm{M}}\end{array}$ & $\begin{array}{c}\mathrm{CLI} 4 \mathrm{~b} \\
{\left[\mathrm{CrO}_{4}{ }^{2-}\right] \mathrm{M}}\end{array}$ & $\begin{array}{c}\text { CLI5 } \\
{\left[\mathrm{CrO}_{4}{ }^{2-}\right] \mathrm{M}}\end{array}$ \\
\hline 0 & $1.25 \mathrm{E}-04$ & $3.05 \mathrm{E}-05$ & $3.74 \mathrm{E}-03$ & $2.41 \mathrm{E}-03$ & $1.56 \mathrm{E}-04$ \\
1 & $2.35 \mathrm{E}-03$ & $4.75 \mathrm{E}-03$ & $3.91 \mathrm{E}-02$ & $3.48 \mathrm{E}-02$ & $1.08 \mathrm{E}-03$ \\
2 & $3.08 \mathrm{E}-03$ & $7.86 \mathrm{E}-03$ & $3.85 \mathrm{E}-02$ & $3.64 \mathrm{E}-02$ & $1.33 \mathrm{E}-03$ \\
4 & $4.20 \mathrm{E}-03$ & $1.16 \mathrm{E}-02$ & $3.92 \mathrm{E}-02$ & $3.67 \mathrm{E}-02$ & $1.62 \mathrm{E}-03$ \\
6 & $5.73 \mathrm{E}-03$ & $1.32 \mathrm{E}-02$ & $3.88 \mathrm{E}-02$ & $3.64 \mathrm{E}-02$ & $1.91 \mathrm{E}-03$ \\
\hline
\end{tabular}





\subsection{References}

Huckaby JL and JR Markillie. 2008. Pretreatment Engineering Platform (PEP) Testing (Phase I). WTP Project Doc. No. 24590-PTF-TSP-RT-07-001, Rev. 2.

Mahoney LA, SD Rassat, PW Eslinger, RL Aaberg, PM Aker, EC Golovich, TS Hausmann, DE Kurath, SK Sundaram, and ST Yokuda. 2009. EFRT Issue Resolution: Caustic Leach Rate Constants from PEP and Lab-Scale Tests. WTP-RPT-186, Rev. 0, PNNL-18502, Pacific Northwest National Laboratory, Richland Washington.

Rapko BM, JR Deschane, GJ Lumetta, and RA Peterson. 2007. Process Development for Permanganate Addition During Oxidative Leaching of Hanford Tank Sludge Simulants. WTP-RPT-164, Rev. 0, PNNL-16794, Pacific Northwest National Laboratory, Richland Washington.

Rapko BA, C Brown, PW Eslinger, MS Fountain, TS Hausmann, and DE Kurath. 2009. EFRT Issue Resolution: Comparison of PEP and Bench-Scale Oxidative Leaching Results. WTP-RPT-188, Rev. 0, PNNL-18500, Pacific Northwest National Laboratory, Richland Washington.

Russell RL, RA Peterson, HD Smith, DE Rinehart, PM Aker, and EC Buck. 2009a. Development and Characterization of Boehmite Component Simulant. WTP-RPT-184, Rev. 1, PNNL-18176, Pacific Northwest National Laboratory, Richland, Washington.

Russell RL, HD Smith, DE Rinehart, and RA Peterson. 2009b. Development and Characterization of Gibbsite Component Simulant. WTP-RPT-176, Rev. 0, PNNL-18013, Pacific Northwest National Laboratory, Richland, Washington.

Russell RL, JM Billing, RA Peterson, DE Rinehart, and HD Smith. 2009c. Development and Demonstration of Ultrafiltration Simulants. WTP-RPT-184, Rev. 0, PNNL-18090, Pacific Northwest National Laboratory, Richland, Washington. 

Appendix A

\section{Boehmite Dissolution Model}





\section{Appendix A: Boehmite Dissolution Model}

Note: This model has been submitted to the International Journal of Chemical Engineering but has not been accepted as of yet.

\section{Boehmite Dissolution Model Based on Simulant Data ABSTRACT}

Several of the Hanford waste tanks contain significant quantities of boehmite. This boehmite will be dissolved through caustic leaching as part of the Hanford Tank Waste Treatment and Immobilization Plant (WTP) currently under construction. Therefore, it is important to fully understand the nature of this dissolution process so that caustic leaching can be effectively deployed on the Hanford tank wastes.

This research determined the impact of primary control parameters such as temperature, hydroxide concentration, approach to solubility, and stirring rate on the boehmite dissolution rate. The impact of aluminate ion on the dissolution kinetics was also determined. In addition, other parameters that impact boehmite dissolution, such as free-hydroxide concentration and reaction temperature, were also assessed and used to develop a semi-empirical model of the boehmite dissolution process. The understanding derived from this work will be used as the basis to evaluate and improve the planned performance of aluminum leaching during waste pretreatment while preparing to vitrify waste in the WTP.

This work is the first in a series of programs aimed at demonstrating the WTP waste pretreatment leaching process. This work was used to develop a simulant of the boehmite-containing Hanford waste. That simulant is subsequently being used in laboratory- and pilot-scale testing to demonstrate the WTP pretreatment process in an integrated fashion.

\section{Introduction}

During the historical production of Pu at the Hanford Site from 1944 to the early 1970s, a significant volume of high-level waste (HLW) sludge was produced and stored in tanks at the Hanford Site. The Hanford Waste Tank Treatment and Immobilization Plant (WTP) under construction on the Hanford Site will be designed to separate the waste into two fractions for immobilization. After the HLW is separated from the low-activity waste (LAW) liquid stream by ultrafiltration in the Pretreatment Facility (PTF), the concentrated HLW will undergo caustic and oxidative leaching processes to dissolve and wash out materials (aluminum, chromium, phosphates, and sulfates) that would otherwise limit HLW loading in the glass waste form. The concentrated HLW solids will be sequentially caustic leached, washed, oxidatively leached, and washed once more during pretreatment. Caustic leaching dissolves the aluminum in the HLW solids, and then oxidative leaching is used to oxidize the chromium with a sodium permanganate $\left(\mathrm{NaMnO}_{4}\right)$ solution and dissolve it in a mild caustic solution. The HLW solids are concentrated after each leaching and washing operation using cross-flow ultrafiltration.

Caustic-leaching experiments were first performed on actual Hanford tank sludge samples in Fiscal Year (FY) 1993. The original caustic-leaching experiments were a prelude to dissolving sludge solids with acid so the acid-dissolved fraction could be processed through solvent extraction. This separates the very small mass fraction of the radioactive elements (the transuranics [TRUs], ${ }^{90} \mathrm{Sr}$, and ${ }^{137} \mathrm{Cs}$ ) from the 
bulk mass of non-radioactive components (Lumetta et al. 1996). In this respect, caustic leaching was meant to remove the large amount of aluminum from the waste, thus reducing the nitric acid demand and simplifying the solvent extraction feed. Subsequently, caustic leaching (sometimes referred to as "Enhanced Sludge Washing) was chosen as the baseline method for Hanford tank sludge pretreatment (Lumetta et al. 1998). Following this decision, caustic-leaching tests were performed under a standard set of conditions at Pacific Northwest National Laboratory (PNNL) and Los Alamos National Laboratory from FY 1994 through FY 1997 (Lumetta et al. 1996, 1997; Rapko et al. 1995; Willingham 1994; Temer and Villarreal 1995, 1996, 1997). In subsequent years, a limited number of parametric caustic-leaching experiments were performed at PNNL and also at Oak Ridge National Laboratory (Lumetta et al. 1998; Palmer et al. 2000; Lumetta et al. 2001; Brooks et al. 1998). After establishing the Hanford WTP project, a limited number of laboratory-scale caustic-leaching experiments were performed using a standard testing protocol (Brooks et al. 2000a, 2000b; Poirier et al. 2003; Russell et al. 2009), but these were generally focused on processing double-shell tank wastes rather than the single-shell tanks where the bulk of the sludge is stored. More recent tests have focused on blends of samples from the single-shell tanks. These tests focused on evaluating the performance of specific chemical species during the causticleaching process.

Caustic-leaching data are needed on the various types of wastes to be processed through the WTP to support the plant design. The data needed include 1) dissolution kinetics of key HLW sludge components (e.g., Al, Cr, P, and S) as a function of caustic concentration, temperature, and time, 2) the behavior of radionuclides during the leaching process, 3) particle-size distribution before and after leaching, and 4) the identification of the chemical and mineral forms of important sludge components (e.g., $\mathrm{Al}, \mathrm{Cr}$, and $\mathrm{P}$ ) in the sludge solids. These data will be used to update the assessments of the expected performance of the WTP pretreatment process and to support the development of various waste simulants for scaled process demonstrations.

Aluminum in the wastes is believed to be present in the two most common mineralogical phases: gibbsite (monoclinic $\mathrm{Al}(\mathrm{OH})_{3}$ ) and boehmite (orthorhombic $\mathrm{AlOOH}$ ). Other Al-containing phases include bayerite, dawsonite, alumina silicates, and amorphous aluminum hydroxide. The dissolution rates of the two primary mineralogical phases are considerably different (Music et al. 1998). Therefore, the leaching kinetics will depend on the relative amounts of these phases in the waste as well as particle size, crystal habit (i.e., particle size and shape), operating temperature, hydroxide activity, aluminum solubility limits, particle Reynolds number associated with the mixing system, etc. The other aluminum compounds in the waste solids are present in relatively smaller amounts and therefore are considered less significant to the caustic leaching for removing aluminum from the HLW.

This appendix describes and evaluates a series of characterization tests that have quantified the various types of aluminum present in the Hanford HLW (Fiskum et al. 2008). Table 1 shows a breakdown of the Al sources in Hanford HLW. Values are shown in terms of the mass of aluminum associated with each phase. The figure indicates that most of the aluminum is either sodium aluminate (supernate and water soluble) or gibbsite. The next major component is boehmite. The boehmite represents the largest component for which aggressive leaching conditions are required to achieve dissolution. As such, understanding the boehmite leaching chemistry and the impacts on the WTP flowsheet using a boehmite simulant will be critical to the WTP performance. 
Table 1. Sources of Al in Hanford Tank Waste

\begin{tabular}{cc}
\hline Al Source & Metric Tons of Al \\
\hline Supernate & 1,188 \\
Water Soluble & 1,297 \\
Easy to Dissolve & 306 \\
Gibbsite & 3,022 \\
Boehmite & 1,775 \\
Unassigned & 568 \\
\hline Intractable & 552 \\
Total & 8,708 \\
\hline
\end{tabular}

A number of studies of boehmite dissolution have been performed in the past (Palmer et al. 2000; Packter 1976; Palmer et al. 2001; Panias 2004). In addition, a number of studies have investigated precipitation kinetics for boehmite (Panias 2004; Skoufadis et al. 2003; Dash et al. 2009). The dissolution studies have indicated, as expected, that the dissolution kinetics are a strong function of both temperature and hydroxide concentration. Packter measured an activation energy for boehmite dissolution between 115 and $125 \mathrm{~kJ} / \mathrm{mole}$. Palmer found that the presence of nitrate appeared to suppress the dissolution of boehmite. Note, however, that none of these studies evaluated the impact of the presence of aluminate ion on the dissolution rate.

\section{Experimental}

This section describes the methods used to conduct the leach testing for both actual waste samples and simulant samples. The data results for the simulant samples are presented in Sections 4 and 5 of the main body of this report.

Boehmite was obtained from APYRAL (for product information, see: Appendix C, product AOH 20), product $\mathrm{AOH}$ 20. X-ray diffraction (XRD) analysis confirmed that this material is boehmite. The tests were performed in a 1-L reaction vessel as shown in Figure 1. The vessel was filled with the leaching fluid and heated to the leaching temperature. The temperature was measured with a calibrated thermocouple (TC) and controlled with a calibrated temperature controller. Boehmite was added as a powder to the reaction vessel through the sample port while stirring after the leaching fluid had reached leaching temperature, which started the clock for the test. The test solution was sampled at 1,2,4,8, and 24 hours. Each sample consisted of 5-mL supernatant, which was filtered through a $0.45-\mu \mathrm{m}$ filter after being drawn from the reaction vessel and then analyzed for aluminum and sodium content by inductive coupled plasma-atomic emission spectroscopy (ICP-AES). The amount of aluminate was adjusted by dissolving gibbsite before introducing the boehmite. The amount of gibbsite added is reflected in the initial aluminate concentration at time 0 for each test. 


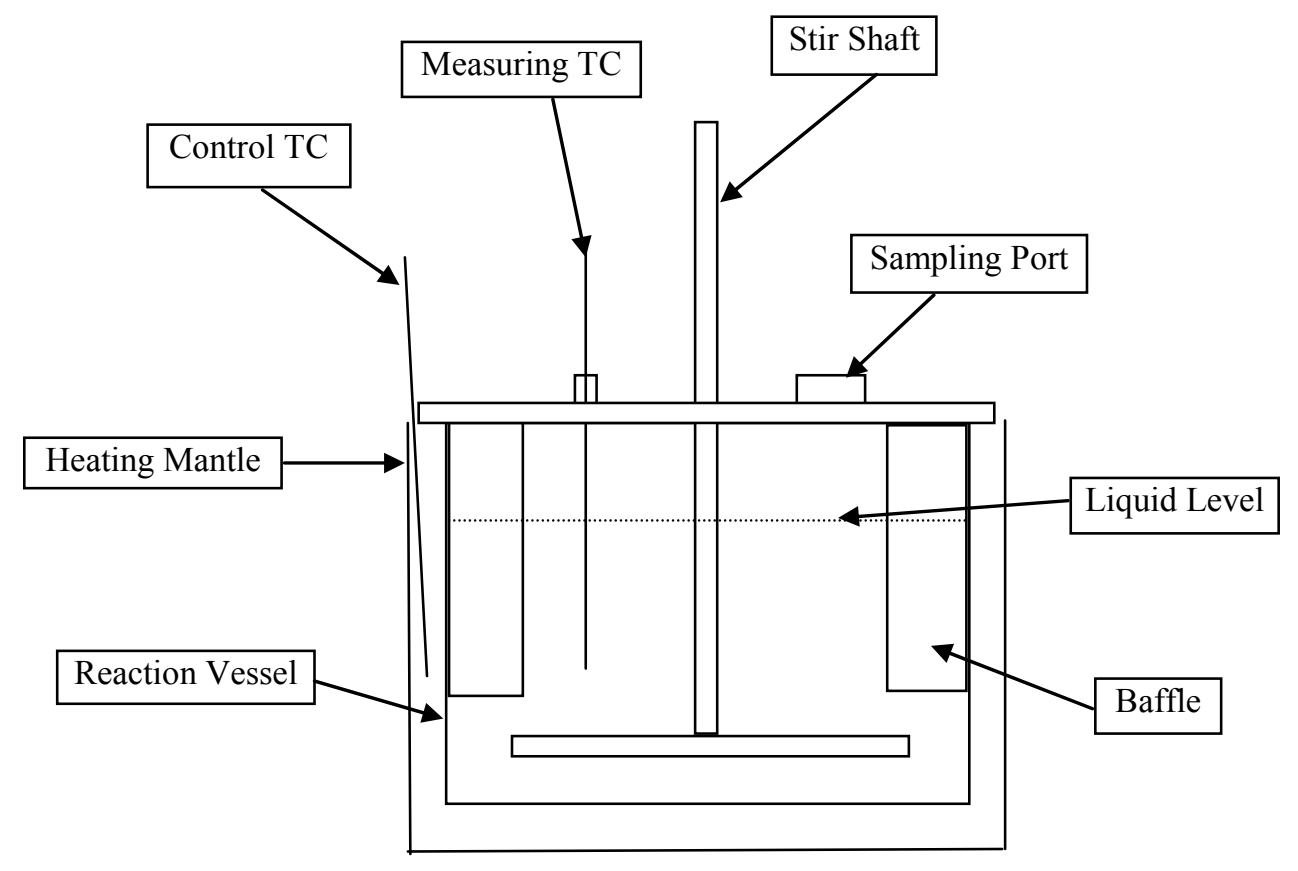

Figure 1. Schematic Drawing of the Caustic Leaching Test Setup

\section{Results}

\section{Sample Characterization}

Figure 2 shows a scanning electron microscopy (SEM) micrograph of the commercially procured boehmite that was used in the simulant tests. Note that the average crystal size for this material is approximately 0.8 microns. The material agglomerates into larger particles, so particle-size distribution measurements do not provide significant insight into the reactivity of the boehmite. 


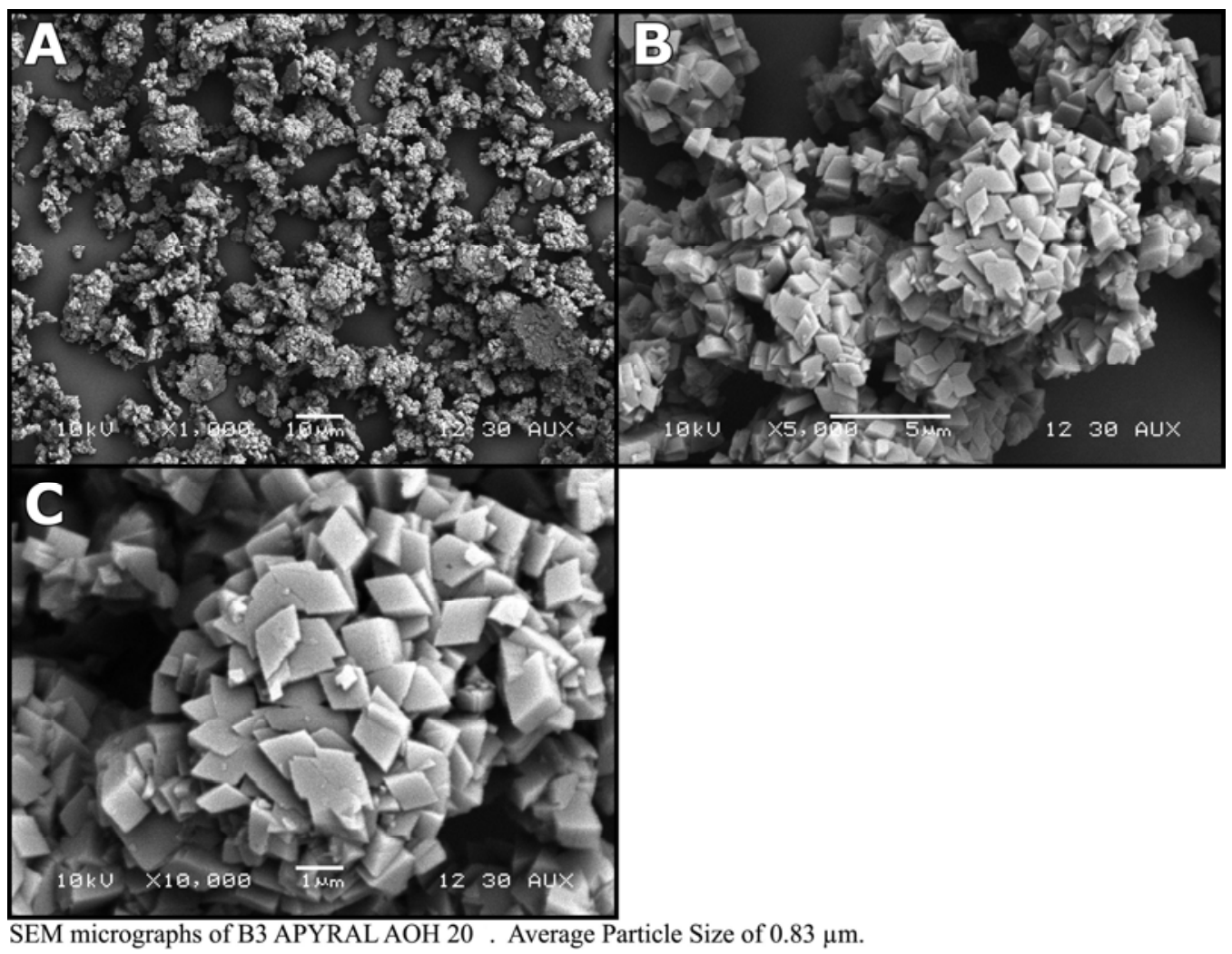

Figure 2. SEM Micrograph of the Boehmite to Be Used in the Simulant

Table 2 compares the surface area of the actual tank waste sample to that of the boehmite used in these tests. As might be expected from the smaller primary particle size, the surface area of the actual tank waste material was significantly larger than for the commercially procured boehmite.

Table 2. Surface Area of Boehmite Samples

\begin{tabular}{ll}
\hline \multicolumn{1}{c}{ Sample ID } & $\begin{array}{c}\text { Specific Surface Area } \\
\left(\mathrm{m}^{2} / \mathrm{g}\right)\end{array}$ \\
\hline Washed Tank Waste Solids & 26 \\
Simulant Boehmite & 10 \\
\hline
\end{tabular}

\section{Aluminum Dissolution Studies}

The dissolution of boehmite is generally expressed as:

$$
\mathrm{AlOOH}+\mathrm{OH}^{-}+\mathrm{H}_{2} \mathrm{O} \Leftrightarrow \mathrm{Al}(\mathrm{OH})_{4}^{-}
$$

At a given condition, this can be written as

$$
\frac{d A l O O H}{d t}=-k_{f}(\mathrm{OH})^{-}+k_{r} \mathrm{Al}(\mathrm{OH})_{4}^{-}
$$


where $\mathrm{k}_{\mathrm{f}}$ includes a surface area term associated with the boehmite surface. At saturation, this can be written as

$\frac{d A l O O H}{d t}=0 \therefore k_{f}(\mathrm{OH})_{s}^{-}=k_{r} \mathrm{Al}(\mathrm{OH})_{4, s}^{-}$

where $s$ represents the concentration at the solubility limit. Substituting produces:

$\frac{d A l O O H}{d t}=-k_{f}(\mathrm{OH})^{-}+k_{f} \frac{(\mathrm{OH})_{s}^{-}}{\mathrm{Al}(\mathrm{OH})_{4, s}^{-}} \mathrm{Al}(\mathrm{OH})_{4}^{-}$

If we assume a relatively large excess of total hydroxide:

$\frac{d A l O O H}{d t}=-k_{f}(\mathrm{OH})^{-}\left(1-\frac{\mathrm{Al}(\mathrm{OH})_{4}^{-}}{\mathrm{Al}(\mathrm{OH})_{4, s}^{-}}\right)$

or

$\frac{d A l O O H}{d t}=-k_{f}(O H)^{-}(1-\sigma)$

where

$\sigma=\frac{\mathrm{Al}(\mathrm{OH})_{4}^{-}}{\mathrm{Al}(\mathrm{OH})_{4, \mathrm{~s}}^{-}}$

then, adding the surface-area dependence

$\frac{d A l O O H}{d t}=-k A_{B}(O H)^{-}(1-\sigma)$

where, based on a shrinking core model (Pereira et al. 2009) where $i$ indicates the initial concentration:

$A_{B} \propto \mathrm{AlOOH}^{2 / 3}$

$\frac{A_{B}}{A_{B . i}}=\left(\frac{A l O O H}{A l O O H_{i}}\right)^{2 / 3}$

$\frac{d\left(\frac{\mathrm{AlOOH}}{\mathrm{AlOOH}_{i}}\right)}{d t}=-k\left(\frac{\mathrm{AlOOH}}{\mathrm{AlOOH}_{i}}\right)^{2 / 3}(\mathrm{OH})^{-}(1-\sigma)$

A set of tests was performed where the initial matrix contained varying amounts of dissolved sodium aluminate. In these tests, the initial supernate contained various levels of soluble aluminate before the start of leaching. The results from these tests are plotted in Figure 3 as a function of the Al concentration 
normalized with $\mathrm{Na}$ to the average value in solution over time at $100^{\circ} \mathrm{C}$. Figure 3 shows that the boehmite dissolves more slowly when more $\mathrm{Al}$ is in the solution. Note that while Equation 11 provides a reasonable fit to the experimental data, it appears to under-predict the impact of the initial aluminate concentration on the reaction rate. This is evidenced by the fact that the model under-predicts at low aluminate and appears to over-predict at high aluminate. This indicates that the dissolution model should be revised. One form that was tested and found to be statistically superior was to include a term for the initial aluminate concentration shown in Equation 12.

$\frac{d\left(\frac{\mathrm{AlOOH}}{\mathrm{AlOOH}_{i}}\right)}{d t}=-k\left(\frac{\mathrm{AlOOH}}{\mathrm{AlOOH}_{i}}\right)^{2 / 3}(\mathrm{OH})^{-}(1-\sigma)\left(1-\sigma_{i}\right)$

This revised model provides a statistically improved fit to the data as seen in Figure 4 . As seen, this revised model now provides more accurate predictions over the entire range of initial aluminate concentrations. Note that simply squaring the last term in Equation $11 \mathrm{did}$ not provide the same fit to the experimental data in that it again led to under-prediction of the reaction rate at low initial aluminate concentrations.

To fit to the $85^{\circ} \mathrm{C}$ data (Figure 5), a temperature correction term is needed. From this data, it was determined that there is a $120-\mathrm{kJ}$ activation energy associated with Equation 13. The data in Figure 5 were used to derive this activation energy. Then the resultant model with the temperature correction included was applied to the data over a range of temperatures and is shown in Figure 6.

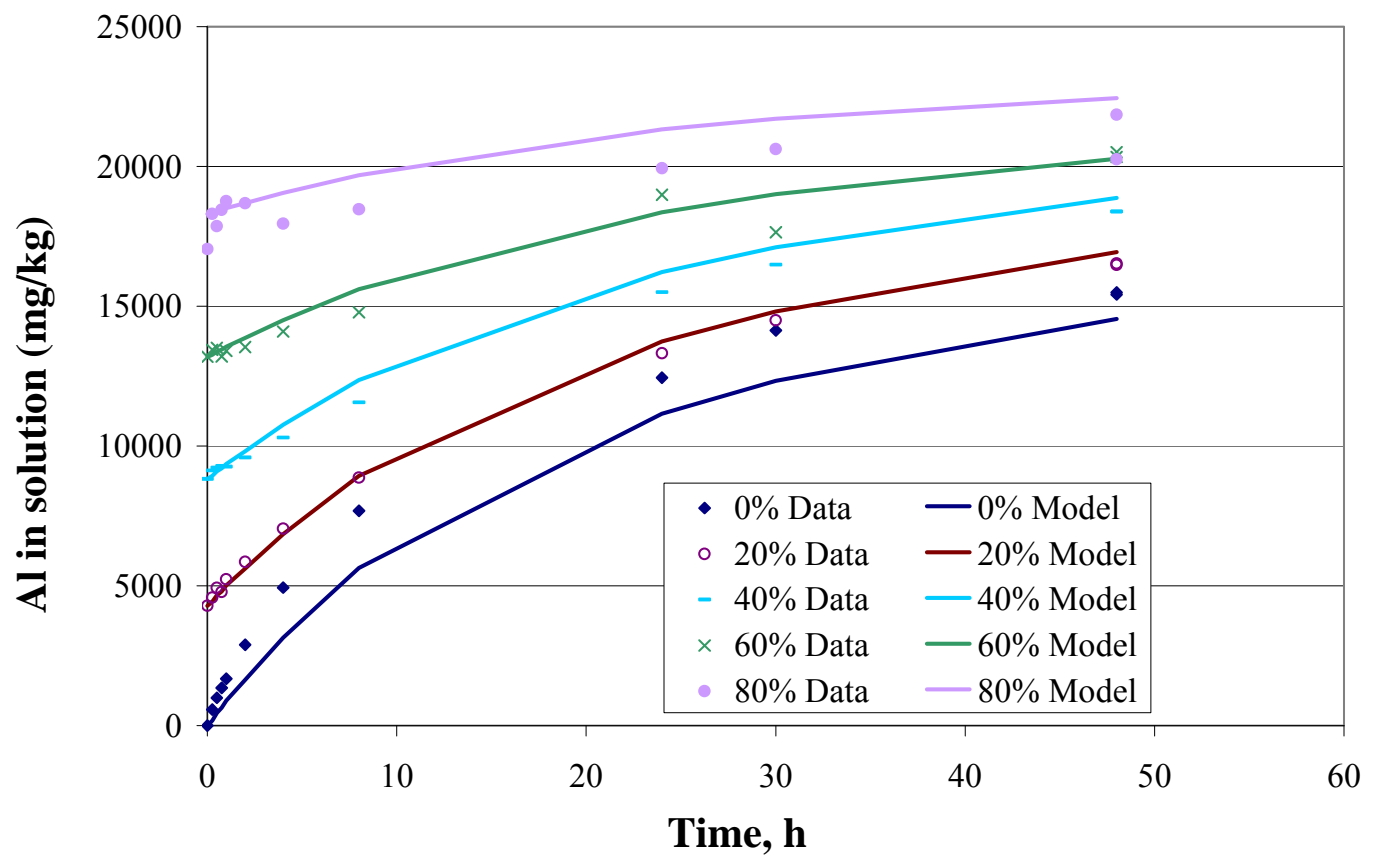

Figure 3. Boehmite Dissolution with Aluminate Present Fit to the Model as Expressed by Eq. 11 


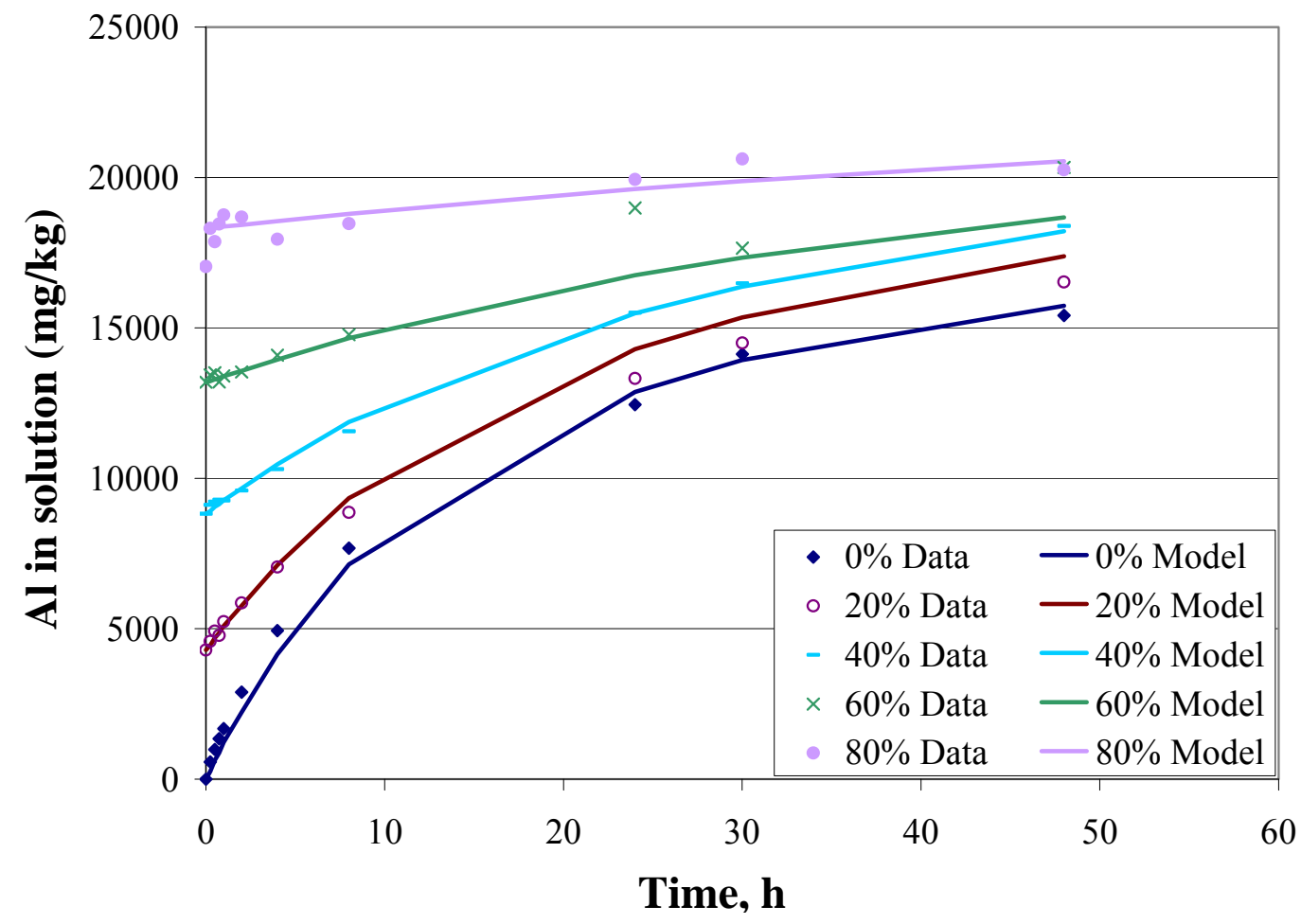

Figure 4. Boehmite Dissolution with Aluminate Present Fit to the Revised Model as Expressed by Eq. 12

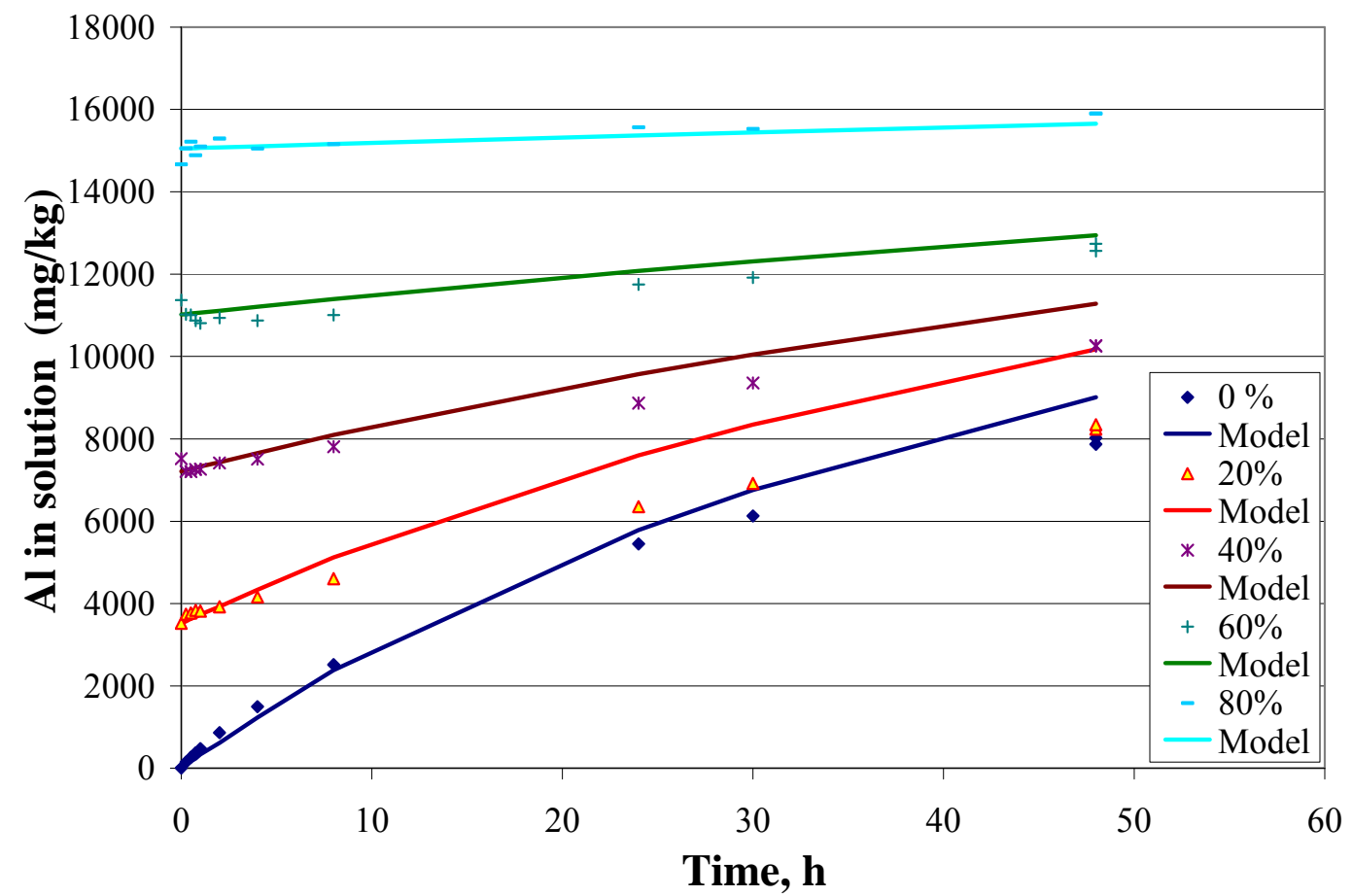

Figure 5. Boehmite Dissolution at $85^{\circ} \mathrm{C}$ Fit to the Model as Expressed by Eq. 13 


$$
\frac{d\left(\frac{\mathrm{AlOOH}}{\mathrm{AlOOH}}\right)}{d t}=-A_{o} e^{-\frac{120,000}{R T}}\left(\frac{\mathrm{AlOOH}}{\mathrm{AlOOH}_{i}}\right)^{2 / 3}(\mathrm{OH})^{-}(1-\sigma)\left(1-\sigma_{i}\right)
$$

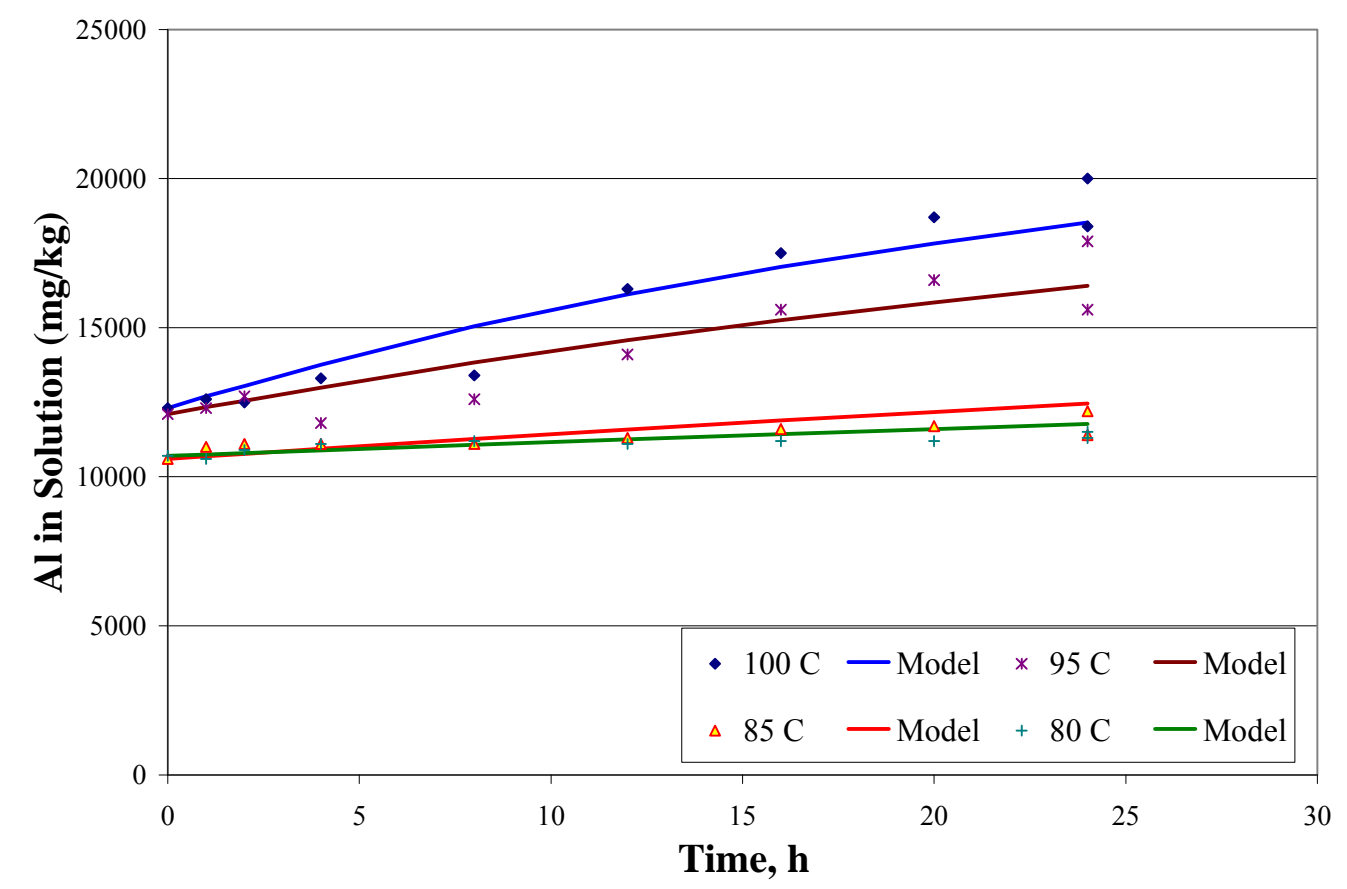

Figure 6. Results from $80^{\circ} \mathrm{C}$ to $100^{\circ} \mathrm{C}$ with Temperature Correction as Expressed by Eq. 13

The impact of varying amounts of gibbsite on boehmite dissolution was evaluated with the derived model. Figure 7 shows a plot of the time necessary to achieve $50 \%$ boehmite dissolution as a function of the Na:Al molar ratio. These results show that the presence of gibbsite requires either more caustic or more time to achieve the same fraction of boehmite dissolved. 


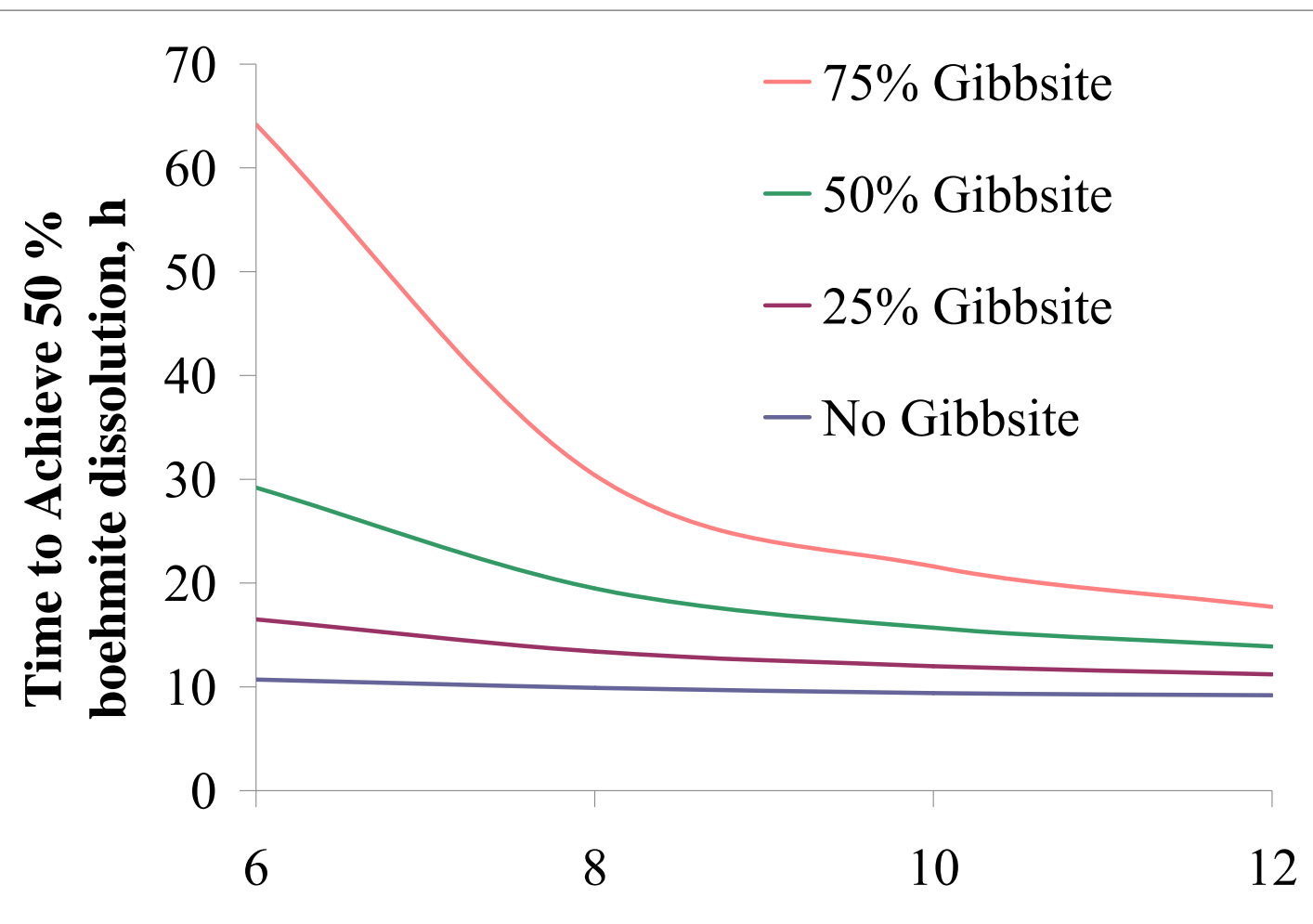

\section{NaOH:Al Molar Ratio}

Figure 7. Impact of Fraction of Aluminum as Gibbsite on Boehmite Dissolution for Various Ratios of $\mathrm{NaOH}$ to Total Insoluble Al

\section{Conclusions}

A shrinking core model was used to fit data from a series of boehmite dissolution tests. An additional term was added to the shrinking core model to account for the approach to saturation. This revised model provided an adequate fit to the experimental data; however, a superior fit to the experimental data was obtained when a term was added to represent the number of dissolution sites available at the start of the reaction as shown in the following equation:

$$
\frac{d\left(\frac{\mathrm{AlOOH}}{\mathrm{AlOOH}_{i}}\right)}{d t}=-A_{o} e^{-\frac{120,000}{R T}}\left(\frac{\mathrm{AlOOH}}{\mathrm{AlOOH}_{i}}\right)^{2 / 3}(\mathrm{OH})^{-}(1-\sigma)\left(1-\sigma_{i}\right)
$$

These results suggest that boehmite will dissolve significantly slower as gibbsite dissolves and adds aluminate to the solution. Practically, these results indicate that the blending wastes with gibbsite and boehmite will ultimately result in either more caustic or more time to achieve the same fraction of boehmite dissolution. For the proposed dissolution process, blending strategies must consider the trade-offs between caustic usage, processing time, and time to prepare feed. 


\section{ACKNOWLEDGEMENTS}

We would like to acknowledge Don Rinehart for all of his lab work in performing the tests and Brian Riley for his SEM work. We would also like to acknowledge Bechtel National, Inc. for funding this work.

\section{References}

Brooks, K. P., J. R. Bontha, G. R. Golcar, R. L. Myers, K. G. Rappe, and D. R. Rector, "Bench-Scale Enhanced Sludge Washing and Gravity Settling of Hanford Tank S-107 Sludge," 1998, PNNL-12010, Rev 0, Pacific Northwest National Laboratory, Richland, Washington.

Brooks, K. P., P. R. Bredt, S. K. Cooley, G. R. Golcar, L. K. Jagoda, K. G. Rappe, and M. W. Urie, "Characterization, Washing, Leaching, and Filtration of AZ-102 Sludge," 2000a, PNWD-3045, Rev 0, Pacific Northwest National Laboratory, Richland, Washington.

Brooks, K. P., P. R. Bredt, G. R. Golcar, S. A. Hartley, L. K. Jagoda, K. G. Rappe, and M. W. Urie, "Characterization, Washing, Leaching, and Filtration of C-104 Sludge," 2000b, PNWD-3024 Rev 0, Pacific Northwest National Laboratory, Richland, Washington.

Dash, B., B. C. Tripathy, I. N. Bhattacharya, S. C. Das, C. R. Mishra, and B. K. Mishra, "Precipitation of boehmite in sodium aluminate liquor," Hydrometallurgy, 2009, 95, 297-301.

Fiskum, S. K., E. C. Buck, R. C. Daniel, K. Draper, M. K. Edwards, T. L. Hubler, L. K. Jagoda, E. D. Jenson, G. J. Lumetta, B. K. McNamara, R. A. Peterson, S. I. Sinkov, and L. A. Snow, "Characterization and Leach Testing for REDOX Sludge and S-Saltcake Actual Waste Sample Composites," 2008, PNNL17368 (WTP-RPT-157), Pacific Northwest National Laboratory, Richland, Washington.

Lumetta, G. J., B. M. Rapko, J. Liu, D. J. Temer, and R. D. Hunt, "Washing and Caustic Leaching of Hanford Tank Sludge: Results of FY 1998 Studies,” 1998, PNNL-12026, Pacific Northwest National Laboratory, Richland, Washington.

Lumetta, G. J., B. M. Rapko, M. J. Wagner, J. Liu, and Y. L. Chen, "Washing and Caustic Leaching of Hanford Tank Sludges: Results of FY 1996 Studies,” 1996, PNNL-11278, Pacific Northwest National Laboratory, Richland, Washington.

Lumetta, G. J., I. Burgeson, M. J. Wagner, J Liu, and Y. L. Chen, "Washing and Caustic Leaching of Hanford Tank Sludge: Results of FY1997 Studies,” 1997, PNNL-11636, Rev 1, Pacific Northwest National Laboratory, Richland, Washington.

Lumetta, G. J., K. J. Carson, L. P. Darnell, L. R. Greenwood, F. V. Hoopes, R. L. Sell, S. I. Sinkov, C. Z. Soderquist, M. W. Urie, and J. J. Wagner, "Caustic Leaching of Hanford Tank S-110 Sludge," 2001, PNNL-13702 Rev 0, Pacific Northwest National Laboratory, Richland, Washington.

Music, S., D. Dragcevic, S. Popovic, and N. Vdovic, "Microstructural Properties of Boehmite Formed Under Hydrothermal Conditions," Materials Science and Engineering B, 1998, 52, 2-3, 145-153. 
Packter, A., "Studies on recrystallised aluminum mono-hydroxide precipitates. Kinetics of dissolution by sodium hydroxide solutions," Colloid and Polymer Science, 1976, 254, 1024-1029.

Palmer, D. A., P. Bénézeth, and D. J. Wesolowski, "Experimental Studies of the Solubilities of Aluminum Oxy-Hydroxy Phases to $300^{\circ} \mathrm{C}$," 2000, Oak Ridge National Laboratory, Oak Ridge, Tennessee.

Palmer, D.A., P. Bénézeth, and D. J. Wesolowski, “Aqueous high-temperature solubility studies. I. The solubility of boehmite as functions of ionic strength (to 5 molal, $\mathrm{NaCl}$ ), temperature $\left(100-290^{\circ} \mathrm{C}\right.$ ), and $\mathrm{pH}$ as determined by in situ measurements," Geochimica et Cosmochimica Acta, 2001, 65, 13, 2081-2095.

Panias, D., "Role of boehmite/solution interface in boehmite precipitation from supersaturated sodium aluminate solutions," Hydrometallurgy, 2004, 74, 203-212.

Pereira, J. A. M., M. Schwaab, E. Dell'Oro, J. C. Pinto, J. L. F. Monteiro, C. A. Henriques, "The kinetics of gibbsite dissolution in $\mathrm{NaOH}$," Hydrometallurgy, 2009, 96, 6-13.

Poirier, M. R., P. Burket, and J. L. Siler, "Filtration, Washing, and Leaching of a Hanford AY-102/C-106 Sample," 2003, WSRC-TR-2003-00240, Rev. 0, Westinghouse Savannah River Company, Aiken, South Carolina.

Rapko, B. M., G. J. Lumetta, and M. J. Wagner, "Washing and Caustic Leaching of Hanford Tank Sludges: Results of FY1995 Studies,” 1995, PNL-10712, Rev 0, Pacific Northwest National Laboratory, Richland, Washington.

Russell, R. L., R. A. Peterson, H. D. Smith, D. E. Rinehart, P. M. Aker, and E. C. Buck, "Development and Characterization of Boehmite Component Simulant," 2009, PNNL-18176, Rev 0, Pacific Northwest National Laboratory, Richland, Washington.

Skoufadis, C., D. Panias, and I. Paspaliaris, "Kinetics of boehmite precipitation from supersaturated sodium aluminate solutions," Hydrometallurgy, 2003, 68, 57-68.

Temer, D. J., and R. Villarreal, "Sludge Washing and Alkaline Leaching Tests on Actual Hanford Tank Sludge: FY1997 Results," 1997, LAUR 97-2889, Rev 0, Los Alamos National Laboratory, Los Alamos, New Mexico.

Temer, D. J., and R. Villarreal, "Sludge Washing and Alkaline Leaching Tests on Actual Hanford Tank Sludge: A Status Report," 1995, LAUR 95-2070 Rev 0, Los Alamos National Laboratory, Los Alamos, New Mexico.

Temer, D. J., and R. Villarreal, "Sludge Washing and Alkaline Leaching Tests on Actual Hanford Tank Sludge: FY1996 Results," 1996, LAUR 96-2839, Rev 0, Los Alamos National Laboratory, Los Alamos, New Mexico.

Willingham, C. E., "Thermophysical Properties of Hanford High-Level Tank Wastes-A Preliminary Survey of Recent Data," 1994, PNL-9419, Rev 0, Pacific Northwest National Laboratory, Richland, Washington. 
Appendix B

Sow for Chromium Hydroxide Preparation 



\title{
Appendix B: SOW for Chromium Hydroxide Preparation
}

\author{
STATEMENT OF WORK \\ Pilot Scale Filtration Simulant Components - Chromium Oxyhydroxide, \\ $\mathrm{CrOOH}$, and Chromium Hydroxide, \\ $\mathrm{Cr}(\mathrm{OH}) 3$
}

July 23, 2007

\section{$\underline{\text { Introduction }}$}

Pacific Northwest National Laboratory (PNNL) is purchasing the components needed to make a non-radioactive filtration simulant. The components include several minerals and chemicals as well as made to order compounds, solutions and slurries. These compounds, solutions and slurries include a supernate, an amorphous Na-Al-Si slurry, an iron-rich sludge slurry and the compounds $\mathrm{CrOOH}$ and $\mathrm{Cr}(\mathrm{OH}) 3$.

Vendors are participating in a two-phase process; this contract is Phase I in which $1 \mathrm{~kg}$ of each compound $\mathrm{CrOOH}$ and $\mathrm{Cr}(\mathrm{OH})_{3}$ is produced, scale-up techniques are determined and the vendor submits an offer for Phase II of the production.

Presently, PNNL is in need of $1 \mathrm{~kg}$ of each compound by September 5, 2007 for immediate testing. Waters of hydration may be included in the mass but unbound waters may not. We are relying on vendors with chemical process expertise and experience. The processing of these two chemicals may be challenging. PNNL chemists have produced the compounds under laboratory conditions and their process techniques have been provided below as an aid. Note that these recipes have only been used to generate relatively small amounts of material needed for laboratory testing. If used, the vendor will need to scale up these procedures. The vendor is allowed to make modifications to these recipes or use alternative methods as long as the specifications are met (specifications are provided below).

Once PNNL receives the compounds they will be tested to determine if the product will be useful for our process. Following the testing, a down selection will be made for a vendor to produce $70 \mathrm{~kg}$ of each compound. As part of Phase I, the vendor is expected to determine how to process this material to make $70 \mathrm{~kg}$ of each material. The vendor will also supply a detailed description of production procedure used, a list of chemicals used including major impurities, and evidence that the specifications have been met. The vendor is expected to provide a quote of the cost for producing the $70 \mathrm{~kg}$ batches.

\section{Preparation of $\mathrm{CrOOH}$}

Four grams (about $0.01 \mathrm{mmol}$ ) of reagent grade $\mathrm{Cr}\left(\mathrm{NO}_{3}\right)_{3} * 9 \mathrm{H} 2 \mathrm{O}$ is placed in $84-\mathrm{mL}$ of water in a Teflon beaker with a Teflon magnetic stirring bar with a graphite bottom suitable for heating on a hot 
plate. Sixteen-mL of $19 \mathrm{M} \mathrm{NaOH}$ is added to the well-stirred solution. The solution initially forms a precipitate, which then redissolves as more base is added. The mixture is stirred and heated to about $90^{\circ} \mathrm{C}$ on the magnetic stirrer/hot plate. When the temperature reaches about $80^{\circ} \mathrm{C}$, a precipitate appears. The system is then heated at about $90^{\circ} \mathrm{C}$ for 2 hours.

The system is allowed to cool overnight and centrifuged. The supernatant is removed by decanting. The residual solids are well mixed with a $100-200-\mathrm{mL}$ of DI water and the centrifuge/decanting cycle repeated for a total of 4 contacts with DI water. The residual solids are then dried under vacuum at ca. $80^{\circ} \mathrm{C}$ for about $72 \mathrm{hrs}$ to yield about 1.04 grams of green solid $(1.03 \mathrm{~g}$ expected).

The green solid is then ground initially with a mortar and pestle followed by 30 minutes in a ball mill "jitterbug" apparatus. After ball milling for 30 minutes, a particle size of about 10 microns is obtained. The final product should be sieved through a 30 -micron mesh sieve to remove large particles. The number of waters (1.0) is determined by thermal gravimetric analysis in air to $600^{\circ} \mathrm{C}$. This reaction has been repeated several times with $[\mathrm{Cr}]$ initially ranging from 0.1 to $0.25 \mathrm{M}$ at various total reaction volumes.

Specifications:

Quantity: $1 \mathrm{~kg}$ dry basis (waters of hydration may be included in mass). Note: unbound water content should be determined either by drying at $105^{\circ} \mathrm{C}$ for 24 hours or by thermal gravimetric analysis.

Particle size: $<30$ microns.

Purity: $>99 \%$ (based on metals content).

Waters of hydration: $1.0 \pm 0.1$ (as determined by thermal gravimetric analysis in air to $600^{\circ} \mathrm{C}$ ).

\section{Preparation of Chromium(III) Hydroxide}

The following two methods have been used to produce $\mathrm{Cr}(\mathrm{III})$ hydroxide:

\section{Method 1. Precipitation from Ammonium Hydroxide}

$\mathrm{CrCl}_{3} * 6 \mathrm{H}_{2} \mathrm{O}$ (35.636 g; 0.134 mole) is dissolved in $75-\mathrm{mL}$ of deionized (DI) water. The resulting solution is filtered through a $0.45-\mu$ m nylon membrane, then it is slowly added (over a period of $\sim 10 \mathrm{~min}$ ) to $150-\mathrm{mL}$ of $4.9 \mathrm{M} \mathrm{NH} 4 \mathrm{OH}$ (prepared by mixing $50-\mathrm{mL}$ of concentrated $\mathrm{NH}_{4} \mathrm{OH}$ solution with 100 -mL DI water) with stirring. After stirring for $\sim 0.5 \mathrm{~h}$, the chromium (III) hydroxide precipitate is filtered using a $0.45-\mu \mathrm{m}$ PES vacuum filter unit. The filtered solid is transferred to a beaker and washed with $200-\mathrm{mL}$ of DI water and filtered again through the PES filter. The washing step is repeated, except that the final filtration is performed using a $0.45-\mu \mathrm{m}$ nylon membrane. The wet chromium (III) hydroxide filter cake is transferred to a watch glass and dried in vacuo over Drierite; the solid is occasionally broken up with a spatula to facilitate drying. (The final dry weight was $18.3 \mathrm{~g}$ of $\mathrm{Cr}(\mathrm{OH})_{3}{ }^{*} \mathrm{xH}_{2} \mathrm{O}$.) The product was size reduced in a ball mill and should be sieved through a 30 micron mesh to remove large particles. The waters of hydration are determined by firing a measured amount of the product to $\mathrm{Cr}_{2} \mathrm{O}_{3}$ in a muffle furnace at $500^{\circ} \mathrm{C}$. The product is formulated as $\mathrm{Cr}(\mathrm{OH}){ }_{3} * 2.4 \mathrm{H}_{2} \mathrm{O}(18.3 \mathrm{~g} ; 0.125$ mole) and the product yield was $93 \%$. (Note: Some 
Cr remained in the original $\mathrm{NH}_{4} \mathrm{OH}$ solution as evidenced by the deep purple color of the filtrate generated when the chromium hydroxide product was filtered. If allowed to stand, a secondary product precipitates from this solution.)

\section{Method 2. Precipitation with Sodium Hydroxide}

$\mathrm{CrCl}_{3} * 6 \mathrm{H}_{2} \mathrm{O}$ (4.933 g; 0.019 mole) is dissolved in 20-mL of deionized (DI) water. Sodium hydroxide solution $(3.251 \mathrm{M}, 17.5-\mathrm{mL}, 0.057 \mathrm{~mole})$ is added drop wise with stirring. After stirring for $45 \mathrm{~min}$, the chromium(III) hydroxide precipitate is filtered using a 0.45 - $\mu \mathrm{m}$ nylon vacuum filter unit. The filtered solid is transferred to a beaker and washed with $20-\mathrm{mL}$ of DI water and filtered again through the nylon filter. The washing step is repeated and the filter containing the wet chromium(III) hydroxide is placed in a vacuum desiccator over Drierite. When the solid is mostly dried, it is transferred to a watch glass, broken up with a spatula, and further dried in vacuo. The final product was size reduced in a ball mill and should be sieved through 30 micron mesh to remove larger particles. (In this case, the waters of hydration were not determined. The final dry weight was $2.2 \mathrm{~g}$ of $\mathrm{Cr}(\mathrm{OH}) 3 * \mathrm{xH} 2 \mathrm{O}$.)

Specifications:

Quantity: $1 \mathrm{~kg}$ dry basis (waters of hydration may be included in mass). Note: unbound water content should be determined either by drying at $105^{\circ} \mathrm{C}$ for 24 hours or by thermal gravimetric analysis.

Particle size: $<30$ microns.

Purity: $>99 \%$ (based on metals content).

Waters of hydration: $2.4 \pm 0.2$ (as determined by thermal gravimetric analysis in air to $600^{\circ} \mathrm{C}$ ). 



\section{Appendix C}

Make Up of PEP Simulant and Component Composition and a Recipe for CrOOH Slurry (from WPT-RPT-201) 



\section{Appendix C: Make Up of PEP Simulant and Component Composition and a Recipe for CrOOH Slurry (from WPT-RPT-201)}

\section{C.1 Tables Giving PEP Simulant Recipes}

Table C.1. Initial PEP Blended Simulant Components (Target of $45 \mathrm{~kg}$ of Simulant)

\begin{tabular}{lll}
\hline & \multicolumn{1}{c}{ Ingredients } & Preparation \\
\hline 1 & $\begin{array}{l}\text { Weigh out } 31700 \text { g of supernate (pre-shimmed to } \\
\text { correct } \mathrm{Na} \text { concentration). }\end{array}$ & Appendix A.2 \\
2 & Add $7910 \mathrm{~g}$ of $5 \mathrm{M} \mathrm{NaOH}$ with mixing. & Commercially available laboratory grade \\
3 & Add 779 g gibbsite with mixing. & Commercially available (Almatis C333) \\
4 & Add 779 g boehmite with mixing. & Commercially available (APYRAL AOH 20) \\
5 & Add 91.4 g Cr oxy-hydroxide slurry with mixing. & Purchased from Noah Chemical \\
6 & Add 225 g sodium oxalate with mixing. & Commercially available laboratory grade \\
7 & Add 3560g iron rich sludge simulant with mixing. & Appendix A.3 \\
8 & Add another 638 g of 5M NaOH with mixing. & Commercially available \\
9 & Actively mix for 1 hour. & \\
\hline
\end{tabular}

Table C.2. PEP Blended Simulant Components with CrOOH Slurry Added Later (Target of $28.5 \mathrm{~kg}$ Simulant)

\begin{tabular}{lll}
\hline & \multicolumn{1}{c}{ Ingredients } & Preparation \\
\hline 1 & $\begin{array}{l}\text { Weigh out 24800 g of supernate (pre-shimmed to } \\
\text { correct Na concentration). }\end{array}$ & Appendix A.2 \\
2 & Add 458 g gibbsite with mixing. & Commercially available (Almatis C333) \\
3 & Add 458 g boehmite with mixing. & Commercially available (APYRAL AOH 20) \\
4 & Add 131 g sodium oxalate with mixing. & Commercially available laboratory grade \\
5 & $\begin{array}{l}\text { Add } 2150 \text { g iron rich sludge simulant with } \\
\text { mixing. }\end{array}$ & Appendix A.3 \\
6 & Add another 379 g of $5 \mathrm{M} \mathrm{NaOH}$ with mixing. & Commercially available laboratory grade \\
7 & Actively mix for 1 hour. & \\
\hline
\end{tabular}

\section{C.2. Preparation of Precipitated Fe-Rich Sludge Solids}

This recipe details the steps to make Precipitated Fe-Rich Sludge Solids. The general steps involved are to dissolve metal nitrates, neutralize these nitrates to form the metal hydroxides, add trim chemicals (phosphate, oxalate, carbonate), then wash the solids with the Simple Supernate for Washing and then with the Supernate. Note that the supernate recipes and sludge washing steps are not included. 
Note: $\leq \pm 0.5 \%$ is sufficient accuracy on masses.

The following preparation should be carried out in a plastic or stainless steel vessel. No glass shall be used. All additions are based on mass.

Note: The target weight of precipitated solids is $\sim 60 \mathrm{~g}$.

Tare weight of 2-L vessel:

1- Add to the 2-L vessel:

\begin{tabular}{ccc}
\hline & Target Mass $(\mathrm{g})$ & Actual Mass $(\mathrm{g})$ \\
\hline Deionized Water & $\sim 300$ & \\
\hline
\end{tabular}

\section{C.2.1 Manganese Dioxide Production}

2- Add to the 2-L vessel:

\begin{tabular}{lccc}
\hline Compounds & Formula & Target Mass $(\mathrm{g})$ & Actual Mass $(\mathrm{g})$ \\
\hline Potassium Permanganate & $\mathrm{KMnO}_{4}$ & $4.37 \pm 0.022$ & \\
\hline
\end{tabular}

Compound should completely dissolve.

3- Add to the 2-L vessel:

\begin{tabular}{lccc}
\hline Compounds & Formula & Target Mass $(\mathrm{g})$ & Actual Mass $(\mathrm{g})$ \\
\hline Manganous Nitrate Solution & $\mathrm{Mn}\left(\mathrm{NO}_{3}\right)_{2}, 50 \mathrm{Wt} \%$ solution & $14.85 \pm 0.074$ & \\
\hline
\end{tabular}

Mix vigorously for $\sim 15$ minutes. It will produce fine black solids which will remain suspended while being agitated.

\section{C.2.2 Preparation of Metal Hydroxides}

4 Add to the 2-L vessel the following transition and other metals compounds with mixing to make sure of complete dissolution (order not of addition not believed important): 


\begin{tabular}{llcc}
\hline Compounds & Formula & Target Mass $(\mathrm{g})$ & Actual Mass $(\mathrm{g})$ \\
\hline Barium Nitrate & $\mathrm{Ba}\left(\mathrm{NO}_{3}\right)_{2}$ & $0.213 \pm 0.001$ \\
Calcium Nitrate & $\mathrm{Ca}\left(\mathrm{NO}_{3}\right)_{2} \cdot 4 \mathrm{H}_{2} \mathrm{O}$ & $2.425 \pm 0.012$ \\
Cadmium Nitrate & $\mathrm{Cd}\left(\mathrm{NO}_{3}\right)$ & $0.06 \pm 0.0003$ \\
Cerium Nitrate & $\mathrm{Ce}\left(\mathrm{NO}_{3}\right)_{3} \cdot 6 \mathrm{H}_{2} \mathrm{O}$ & $0.65 \pm 0.003$ \\
Copper Nitrate & $\mathrm{Cu}\left(\mathrm{NO}_{3}\right)_{2} \cdot 3 \mathrm{H}_{2} \mathrm{O}$ & $0.157 \pm 0.0008$ \\
Ferric Nitrate & $\mathrm{Fe}\left(\mathrm{NO}_{3}\right)_{3} \cdot 9 \mathrm{H}_{2} \mathrm{O}$ & $128.1 \pm 0.64$ \\
Lanthanum Nitrate & $\mathrm{La}\left(\mathrm{NO}_{3}\right)_{3} \cdot 6 \mathrm{H}_{2} \mathrm{O}$ & $0.482 \pm 0.002$ \\
Lead Nitrate & $\mathrm{Pb}\left(\mathrm{NO}_{3}\right)_{2}$ & $1.295 \pm 0.006$ \\
Magnesium Nitrate & $\mathrm{Mg}\left(\mathrm{NO}_{3}\right)_{2} \cdot 6 \mathrm{H}_{2} \mathrm{O}$ & $1.72 \pm 0.009$ \\
Neodymium Nitrate & $\mathrm{Nd}\left(\mathrm{NO}_{3}\right)_{3} \cdot 6 \mathrm{H}_{2} \mathrm{O}$ & $1.32 \pm 0.007$ \\
Nickel Nitrate & $\mathrm{Ni}\left(\mathrm{NO}_{3}\right)_{2} \cdot 6 \mathrm{H}_{2} \mathrm{O}$ & $2.87 \pm 0.014$ \\
Praseodymium Nitrate & $\mathrm{Pr}\left(\mathrm{NO}_{3}\right)_{3} \cdot \times \mathrm{H}_{2} \mathrm{O} \times \mathbf{x}$ & $0.33 \pm 0.002$ \\
Ruthenium Trichloride & $\mathrm{RuCl}$ & $0.11 \pm 0.0005$ \\
Silver Nitrate & $\mathrm{AgNO}$ & $0.486 \pm 0.002$ \\
Strontium Nitrate & $\mathrm{Sr}\left(\mathrm{NO}_{3}\right)_{2}$ & $0.347 \pm 0.002$ \\
Yttrium Nitrate & $\mathrm{Y}\left(\mathrm{NO}_{3}\right)_{3} \cdot 6 \mathrm{H}_{2} \mathrm{O}$ & $0.14 \pm 0.0007$ \\
Zinc Nitrate & $\mathrm{Zn}\left(\mathrm{NO}_{3}\right)_{2} \cdot 6 \mathrm{H}_{2} \mathrm{O}$ & $0.169 \pm 0.0008$ \\
Zirconyl Nitrate & $\mathrm{ZrO}\left(\mathrm{NO}_{3}\right)_{2} \cdot x \mathrm{H}_{2} \mathrm{O} \times 6$ & $1.73 \pm 0.009$ \\
Mercuric Nitrate & $\mathrm{Hg}\left(\mathrm{NO}_{3}\right)_{2}$ & $0.052 \pm 0.0003$ \\
\hline
\end{tabular}

5 Mix vigorously to completely dissolve everything except the fine black solids of $\mathrm{MnO}_{2}$. A little DIW may be added if necessary for complete dissolution to occur.

DIW water added:

\section{C.2.3 Neutralization of Nitrate Solution}

6 Standardize a pH electrode with $\mathrm{pH} \mathrm{4,} 7$ and 10 buffers.

$\mathrm{pH} 4$ buffer:

Manufacturer:

Lot\#:

Exp Date:

pH 7 buffer:

Manufacturer:

Lot\#:

Exp Date:

pH 10 buffer:

Manufacturer:

Lot\#:

Exp Date:

7 Place the $\mathrm{pH}$ electrode in the precipitation vessel with the metal nitrates and measure the $\mathrm{pH}$.

$\mathrm{pH}:$

Note: $\mathrm{pH}$ should be $<1$.

With the nitrate solution agitating, slowly add $8 \mathrm{M} \mathrm{NaOH}$, until the $\mathrm{pH}$ reaches 10 to 11 . Estimated amount of $8 \mathrm{M} \mathrm{NaOH}$ needed is $190 \mathrm{~g}$. 
8 Measure the $\mathrm{pH}$.

$\mathrm{pH}:$

9 Continue mixing for 1 hour and then recheck $\mathrm{pH}$.

$\mathrm{pH}$ :

10 Add additional $8 \mathrm{M} \mathrm{NaOH}$ to return the $\mathrm{pH}$ to 10 if it is lower.

Total $8 \mathrm{M} \mathrm{NaOH}$ added:

Final $\mathrm{pH}$ :

\section{C.2.4 Addition of Additional Reagents}

11 Add to the 2-L vessel:

\begin{tabular}{llcc}
\hline Compounds & Formula & Target Mass (g) & Actual Mass (g) \\
\hline Calcium Fluoride & $\mathrm{CaF}_{2}$ & $0.205 \pm 0.001$ & \\
Sodium Phosphate & $\mathrm{Na}_{3} \mathrm{PO}_{4} \cdot 12 \mathrm{H}_{2} \mathrm{O}$ & $5.05 \pm 0.03$ & \\
\hline
\end{tabular}

12 Combine the following in a separate $250-\mathrm{mL}$ container while stirring:

\begin{tabular}{lccc}
\hline Compound & Formula & Target Mass $(\mathrm{g})$ & Actual Mass $(\mathrm{g})$ \\
\hline Water (deionized) & & $\sim 100$ & \\
Sodium Oxalate & $\mathrm{Na}_{2} \mathrm{C}_{2} \mathrm{O}_{4}$ & $6.7 \pm 0.03$ & \\
\hline
\end{tabular}

Add this sodium oxalate solution to the 2-L vessel while stirring.

13 Combine the following in a separate $250-\mathrm{mL}$ container while stirring:

\begin{tabular}{lccc}
\hline Compound & Formula & Target Mass (g) & Actual Mass (g) \\
\hline Water (deionized) & & $\sim 100$ & \\
Sodium Carbonate & $\mathrm{Na}_{2} \mathrm{CO}_{3}$ & $9.50 \pm 0.05$ & \\
\hline
\end{tabular}

Add this sodium carbonate solution to the $2-\mathrm{L}$ vessel with stirring. Approximate volume at this point should be about $0.9 \mathrm{~L}$.

14 Mix (vigorously) the slurry to ensure good mixing. Mix the slurry for at least 1 hour.

\section{C.3 APYRAL Boehmite Product Description}

The mineral powder described below is added to the actively mixing slurry in the amount specified in the recipe. 


\section{APYRAL ${ }^{\circledR}$ \\ Provisional Product Information APYRAL AOH 20, APYRAL AOH 20Y, APYRAL AOH 60}

Mineral flame retardants for

- Wire and cable industry

- Electronic industry

- Public Transport

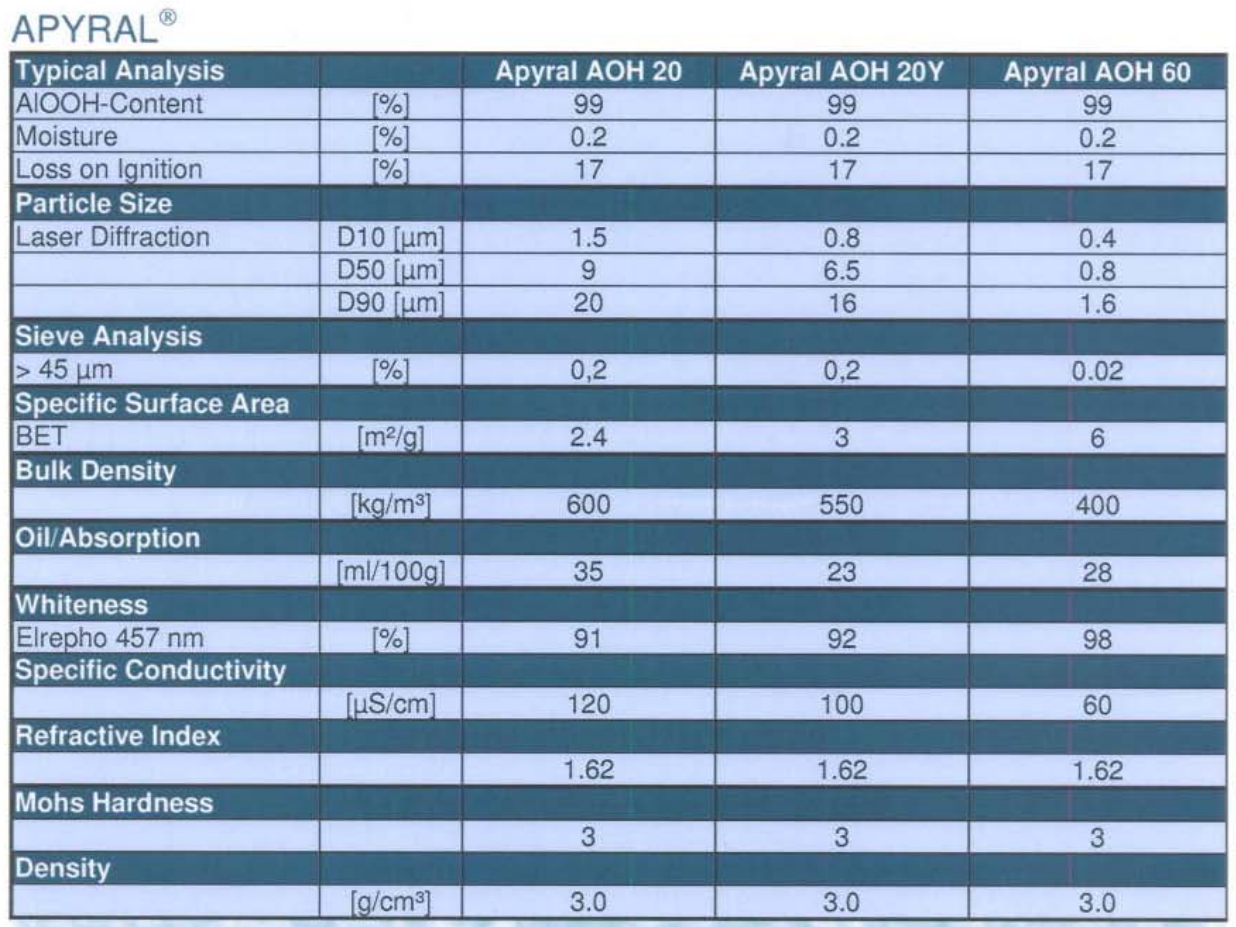

Produktvorteile / Product properties

High temperature stability up to $320^{\circ} \mathrm{C}$ for high temperature applications

- Various particle size distributions for different applications

Low sedimentation and good viscosity performance in resins

Good char promoter

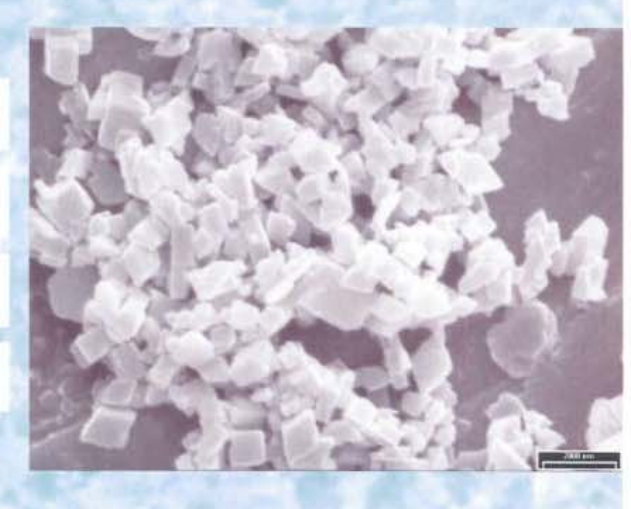

All this Data are provisional and only for information of the user. They do not describe legally binding properties. It remains the responsibility of the users to test the suitability of the products for the application.

\section{C.4 Gibbsite Product Description}

The mineral powder described below is added to the actively mixing slurry in the amount specified in the recipe. 


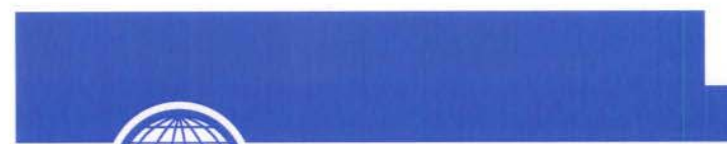

\section{Global Product Data}

\section{Alumina Trihydroxides Exceptionally Pure White Hydrates}

\section{Product Information}

Almatis white hydrated alumina is aluminum trihydroxide, $\mathrm{Al}(\mathrm{OH})_{3}$, that is produced through special processing of alumina-bearing feedstocks and stringent process control systems. The result is an aluminum trihydroxide of exceptional purity and whiteness. Although aluminum trihydroxide is a dry powder, it contains a high proportion, approximately 35 percent by weight, of chemically combined water. The hydrate is a nonabrasive, low-density material with a Mohs hardness index of 2.5-3.5 and a specific gravity of 2.42. White hydrates are used primarily in applications where color and the absence of impurities are critical. They are halogen-free making them excellent nontoxic flame retardant/smoke suppressant fillers for plastic compounds.

\section{Product Description}

Almatis precipitates a highly pure gibbsite phase of alpha alumina trihydrate. The Almatis proprietary white stream process is designed, through chemical and recrystallization processes, to achieve near 100 percent photovolt brightness and relatively uniform particles.

\section{C-33 and C-31C (coarse)}

The precipitation process is controlled to produce two median particle sizes, Grades C-33 (50 microns) and C-31C (85 microns). Both grades have free-flowing properties.

\section{C-333 Ground White Hydrates}

A fine size grade is produced by grinding the precipitated grade to form C-333 (7 microns).

\section{Applications}

Grades C.33 and C.31C hydrates are used in the manufacture of glass, chemicals, catalysts, vitreous enamels and ceramic whitewares, and as additives in high quality pigments. These products are also used as additives and fillers in polymer systems such as electrical wire insulation and high quality cultured onyx and solid countertop surfacing material. Aluminum trihydroxides are preferred because of their good arc and track resistance, aesthetic properties, reinforcing characteristics, and performance as nontoxic smoke suppressants and flame retardants.

C-333 is a ground version of the precipitated grade. It is used in polymer formulations, toothpastes, adhesives, coatings, paper, cosmetics, waxes, and polishes.

Think alumina, think Almatis. 


\section{Alumina Trihydroxides Exceptionally Pure White Hydrates}

\begin{tabular}{|c|c|c|c|c|}
\hline \multirow[t]{2}{*}{ Product } & \multicolumn{3}{|c|}{ Aluminum Trihydroxides } & \multirow[b]{2}{*}{ Test Methods } \\
\hline & $(-33$ & $C-31 C$ & $C-333$ & \\
\hline \multicolumn{5}{|l|}{ Chemical Composition (\%) } \\
\hline $\mathrm{Al}(\mathrm{OH})_{3}(\mathrm{~min})$ & 99.6 & 99.6 & 99.6 & Difference \\
\hline $\mathrm{SiO}_{2}$ & 0.003 & 0.003 & 0.003 & \multirow{3}{*}{$\begin{array}{l}\text { DC Arc Optical } \\
\text { Emission Spectrometry }\end{array}$} \\
\hline $\mathrm{Fe}_{2} \mathrm{O}_{3}$ & 0.009 & 0.009 & 0.009 & \\
\hline $\mathrm{Na}_{2} \mathrm{O}$ (total) & 0.20 & 0.26 & 0.17 & \\
\hline $\mathrm{Na}_{2} \mathrm{O}$ (soluble) & 0.008 & 0.008 & 0.026 & Hame Emission Photometry \\
\hline Moisture & 0.08 & 0.08 & 0.26 & Microwave \\
\hline \multicolumn{5}{|l|}{ Physical Properties } \\
\hline Loose bulk density $\left(\mathrm{g} / \mathrm{cm}^{3}\right)$ & 1.07 & 1.15 & 0.76 & Modified ASTM B212-89 \\
\hline Packed bulk density $\left(\mathrm{g} / \mathrm{cm}^{3}\right)$ & 1.3 & 1.3 & 1.15 & Modified ASTM B527-85 \\
\hline Refractive index & 1.57 & 1.57 & 1.57 & \\
\hline Mohs hardness & $2.5-3.5$ & $2.5-3.5$ & $2.5-3.5$ & \\
\hline Density $\left(\mathrm{g} / \mathrm{cm}^{3}\right)$ & 2.42 & 2.42 & 2.42 & \\
\hline Surface area $\left(m^{2} / g\right)$ & . & - & 3.0 & BET \\
\hline LOI (\%) & 34.6 & 34.6 & 34.6 & From $110-1100^{\circ} \mathrm{C}$ \\
\hline Color & White & White & White & \\
\hline \multicolumn{5}{|l|}{ Particle Size Distribution } \\
\hline$\%$ on 100 Tyler mesh & 0 & 0 & . & \\
\hline$\%$ on 200 Tyler mesh & 8 & 45 & 0 & \\
\hline$\%$ on 325 Tyler mesh & 60 & 90 & 1 & \\
\hline$\%$ through 325 Tyler mesh & 40 & 10 & 99 & Wet Screen \\
\hline$d 50(\mu)$ & 50 & 85 & 7 & Sedigraph 5100 or Microtrac \\
\hline
\end{tabular}

2003 Data - All data are bosed upon Almatis standard test methods, and all test methods are available upon request. Unless stated otherwise values are typical. 


\section{C.5 Preparation of Chromium Oxyhydroxide (CrOOH) Slurry}

\section{General}

The following recipe, which assumes $100 \% \mathrm{Cr}$ solids yield, will provide $73.2 \mathrm{~kg} \mathrm{Cr}$ as $\mathrm{CrOOH}$ in a nominal $2 \underline{\mathrm{M}}$ hydroxide slurry. Past characterizations suggest that the UDS is nominally $\mathrm{CrOOH}-\mathrm{H}_{2} \mathrm{O}$.

\section{Recipe}

1) Completely dissolve $140.8 \mathrm{~kg}$ of $\mathrm{Cr}\left(\mathrm{NO}_{3}\right)_{3}-9 \mathrm{H}_{2} \mathrm{O}$ in $1410 \mathrm{~kg}$ DIW with stirring. The DIW to $\mathrm{Cr}\left(\mathrm{NO}_{3}\right)_{3}-9 \mathrm{H}_{2} \mathrm{O}$ mass ratio is $10: 1$ is critical. Use a saturated $\mathrm{Cr}\left(\mathrm{NO}_{3}\right)_{3}-9 \mathrm{H}_{2} \mathrm{O}$ solution $(77-\mathrm{wt} \%$ $\left.\mathrm{Cr}\left(\mathrm{NO}_{3}\right)_{3}-9 \mathrm{H}_{2} \mathrm{O}\right)$ and dilute rather than beginning with a solid reagent to make certain that all the chromium is in solution.

2) Slowly add $203 \mathrm{~kg}$ of NaOH in the form of a $0.76 \mathrm{~g} \mathrm{NaOH} / \mathrm{g}$ DIW solution (estimated $15.8 \underline{\mathrm{M}}$ $\mathrm{NaOH}$ ) with continued mixing of the solution while maintaining the temperature below $60^{\circ} \mathrm{C}$.

Note: A precipitate should form during $\mathrm{NaOH}$ addition but should redissolve as more caustic is added.

3) After all the solids have redissolved, heat the mixture to $90^{\circ} \mathrm{C}$ within 1 to $2 \mathrm{~h}$, while mixing.

Note: When the temperature reaches about $80^{\circ} \mathrm{C}$, a precipitate should appear.

4) Maintain temperature at $90^{\circ} \mathrm{C}$ for 2 hours while mixing.

5) Actively cool the slurry slowly to ambient temperature $\left(\sim 19^{\circ} \mathrm{C}\right)$ in 8 to 16 hours while mixing. Monitor the temperature during cooling.

6) Measure the UDS and the $\left[\mathrm{OH}^{-}\right]$by titration to its first equivalence point.

\section{Batch Characterization}

1) Report the measured PSD, UDS, hydroxide, and the common anions' (nitrate, nitrite, chloride, sulfate, phosphate, and oxalate) concentrations typically obtained using IC or an equivalent method and inorganic carbon.

2) Measure the metals content using ICP/OES or equivalent method. The elements to be reported include $\mathrm{Al}, \mathrm{B}, \mathrm{Ba}, \mathrm{Bi}, \mathrm{Ca}, \mathrm{Ce}, \mathrm{Cr}, \mathrm{Fe}, \mathrm{K}, \mathrm{La}, \mathrm{Mg}, \mathrm{Mn}, \mathrm{Pb}, \mathrm{Nd}, \mathrm{Ni}, \mathrm{P}, \mathrm{Sr}, \mathrm{S}, \mathrm{Si}, \mathrm{Sr}, \mathrm{Zn}$, and Zr. 
PNNL-18597

WTP-RPT-200, Rev 0

\section{Distribution}

No. of

Copies

OFFSITE
No. of

Copies

\section{ONSITE}

$1 \quad$ Pacific Northwest National Laboratory (Authors will be notified electronically)

W.C. Buchmiller

K6-24

D.E. Kurath

K3-52

L.A. Mahoney

K7-15

R.A. Peterson

P7-22

B.M. Rapko

P7-25

D.E. Rinehart

K6-24

R.L. Russell

K6-24

Information Release (pdf)

Project File (1)

K3-52

4 Bechtel National, Inc.

WTP R\&T Docs (2)

H4-02

S.M. Barnes

H4-02

J.L. Huckaby

H4-02

Distr. 1 


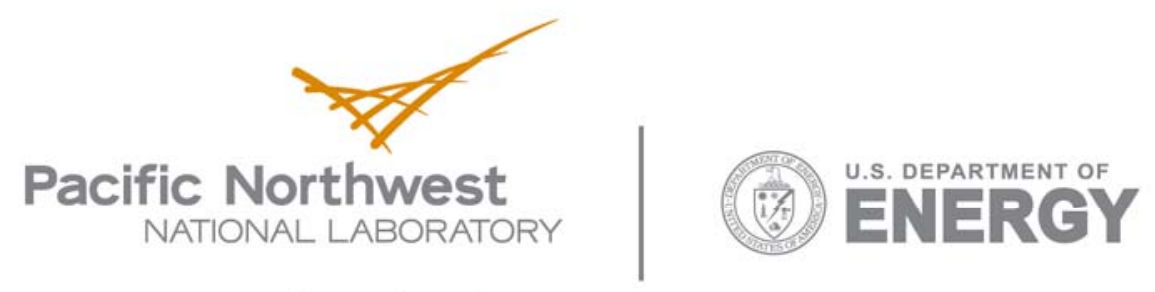

902 Battelle Boulevard

P.O. Box 999

Richland, WA 99352

1-888-375-PNNL (7665)

www.pnl.gov 